\title{
Palladium/Copper-Catalyzed Denitrogenative Alkylidenation and ortho-Alkynylation Reaction of 1,2,3-Benzotriazin-4(3H)-ones
}

\author{
Madasamy Hari Balakrishnan ${ }^{a}$ and Subramaniyan Mannathan ${ }^{b}$
}

${ }^{a}$ Department of Chemistry, SRM Institute of Science and Technology, Kattankulathur, Chennai 603203, India

${ }^{b}$ Department of Chemistry, SRM University-AP, Amaravati 522502, Andhra Pradesh, India

\section{Supporting Information}

\section{Table of Contents}

Page S2 - S6

Page S6

Page S6

Page S7-S25

Page S26- S38
Experimental Section

Optimization studies

References

${ }^{1} \mathrm{H}$ NMR, ${ }^{13} \mathrm{C}$ NMR, and HRMS data

Single crystal XRD data 


\section{Experimental Section}

\section{Materials and methods}

All experiments were carried out in oven-dried glassware, in an atmosphere of argon, unless specified otherwise, by standard Schenk techniques. Flash column chromatography was performed on 100-200 mesh silica gel. ${ }^{1} \mathrm{H}$ and ${ }^{13} \mathrm{C}$ NMR spectroscopy were performed on Bruker BBFO (500 MHz) spectrometer. Chemical shifts were determined relative to the residual solvent peaks $\left(\mathrm{CHCl}_{3}, \delta=7.26 \mathrm{ppm}\right.$ for ${ }^{1} \mathrm{H} \mathrm{NMR}, \delta=77.0 \mathrm{ppm}$ for $\left.{ }^{13} \mathrm{C} \mathrm{NMR}\right)$. The mass spectra (ESI-MS) were recorded on an Agilent 6230 Series TOF LC/MS spectrometer. X-ray crystallography analysis was performed on Bruker X8 APEX X-ray diffraction meter. The starting materials, Benzotriazin-4(3H)-ones $\mathbf{1} \mathbf{a}-\mathbf{k}^{1-2}$ and $\mathbf{5} \mathbf{a a}^{3}{ }^{3}$ were prepared according to a literature procedure. All acetylenes 2 were purchased from Spectrochem, Sigma Aldrich, and Alfa Aesar, and used. All the solvents used for reactions and extraction, filtration, and flash chromatography were purchased from Fisher scientific and used without further purification. Palladium(II) acetate, and $\mathrm{Cu}(\mathrm{I})$ salts were purchased from Sigma Aldrich and used. Ligands such as Xantphos, $\mathrm{PPh}_{3}$, dppp, dppm were purchased form Alfa Aesar or Avra and used without further purification.

\section{General preparation of (Z)-3-benzylidene-2-arylisoindolin-1-one (3)}<smiles>[R][R]1ccc(-n2nnc3cc[R1]cc3c2=O)cc1</smiles>

1

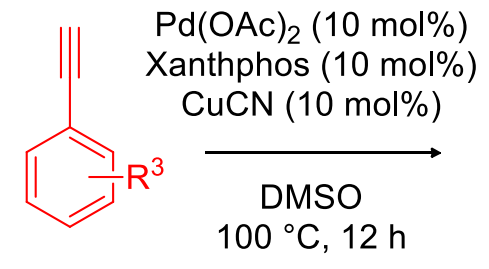

2

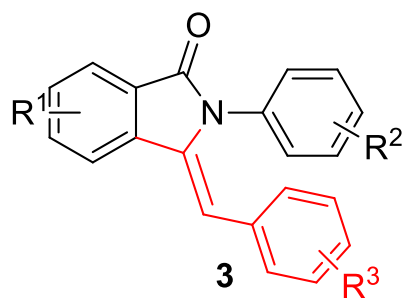

A sealed tube containing 1,2,3-Benzotriazin-4(3H)-ones $1(0.40 \mathrm{mmol})$, aryl acetylene 2 (0.60 mmol), $\mathrm{Pd}(\mathrm{OAc})_{2}(0.04 \mathrm{mmol})$, Xantphos $(0.04 \mathrm{mmol})$ and $\mathrm{CuCN}(0.04 \mathrm{mmol})$ was evacuated and purged with Argon gas three times at room temperature. To that, anhydrous DMSO ( $2 \mathrm{~mL})$ was added and stirred for 2 minutes at room temperature. The sealed tube was then placed in a preheated oil bath at $100{ }^{\circ} \mathrm{C}$ for $12 \mathrm{~h}$. After completion of the reaction, the mixture was cooled and diluted with dichloromethane $(10 \mathrm{~mL})$. The mixture was filtered through a Celite and silica gel pad and was washed with dichloromethane $(3 \times 10 \mathrm{~mL})$. The reaction mass was poured into $15 \mathrm{~mL}$ of water and shaken well. The organic layer was separated, dried on anhydrous sodium sulphate bed and concentrated under vacuum. The residue was purified by silica gel column chromatography using suitable eluent to afford the desired pure product 3 . 
Procedure for the synthesis of (Z)-3-benzylidene-2-(4-methoxyphenyl)isoindolin-1-one (3aa) in a gram scale<smiles>COc1ccc(-n2nnc3ccccc3c2=O)cc1</smiles>

1a

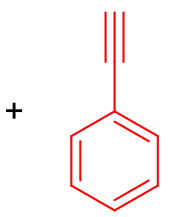

$2 \mathbf{a}$

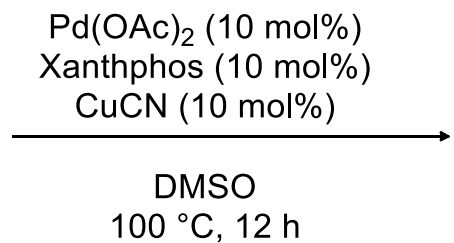

$100{ }^{\circ} \mathrm{C}, 12 \mathrm{~h}$

A sealed tube containing 3-(4-methoxyphenyl)benzo[d][1,2,3]triazin-4(3H)-one 1a $(1.0 \mathrm{~g}, 4.00 \mathrm{mmol})$, phenyl acetylene $2 \mathrm{a}(612 \mathrm{mg}, 6.00 \mathrm{mmol}), \mathrm{Pd}(\mathrm{OAc})_{2}(44 \mathrm{mg}, 0.20$ mmol), Xantphos (116 mg, $0.20 \mathrm{mmol}$ ), and $\mathrm{CuCN}$ (36 mg, $0.40 \mathrm{mmol}$ ) was evacuated and purged with Argon gas three times at room temperature. To that, anhydrous DMSO $(2 \mathrm{~mL})$ was added and stirred for 2 minutes at room temperature. The sealed tube was then placed in a preheated oil bath at $100{ }^{\circ} \mathrm{C}$ for $12 \mathrm{~h}$. The sealed tube was then placed in a preheated oil bath at $100{ }^{\circ} \mathrm{C}$ for $12 \mathrm{~h}$. After completion of the reaction, the mixture was cooled and diluted with dichloromethane $(30 \mathrm{~mL})$. The mixture was filtered through a Celite and silica gel pad and was washed with dichloromethane $(3 \times 15 \mathrm{~mL})$. The reaction mass was poured into 50 $\mathrm{mL}$ of water and shaken well. The organic layer was separated and concentrated under vacuum. The residue was purified by silica gel column chromatography using hexane:ethylacetate (85:15) as eluent to afford the desired pure product 3aa (1.178 g yield; $90 \%)$.

General preparation of (Z)-3-benzylideneisobenzofuran-1(3H)-ylidene)arylanilines (4)<smiles>[R][R]1ccc(-n2nnc3cc#[R1]cc3c2=O)cc1</smiles>

1
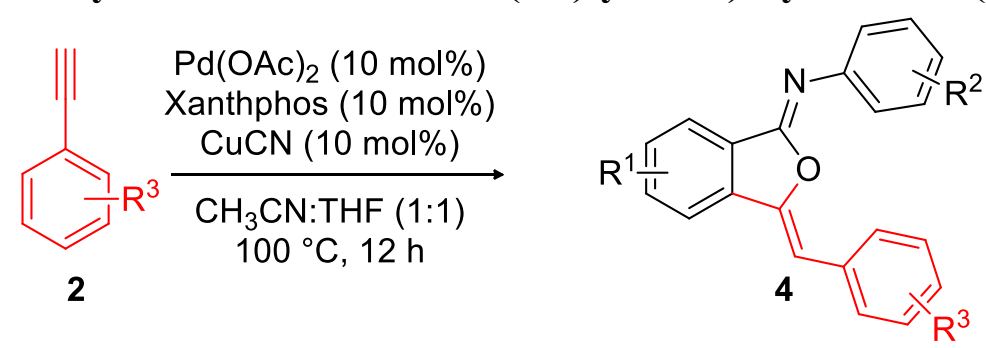

A sealed tube containing 1,2,3-benzotriazin-4(3H)-ones 1 ( $0.40 \mathrm{mmol}, 1$ equiv), aryl acetylene 2 ( $0.60 \mathrm{mmol}, 2$ equiv), $\mathrm{Pd}(\mathrm{OAc})_{2}$ ( $0.04 \mathrm{mmol}, 0.1$ equiv), Xantphos (0.04 mmol, 0.10 equiv), and $\mathrm{CuCN}$ (0.04 mmol, 0.10 equiv) was evacuated and purged with Argon gas three times at room temperature. To that, anhydrous $\mathrm{CH}_{3} \mathrm{CN}$ :THF $(1: 1)(2 \mathrm{~mL})$ was added and stirred for 2 minutes at room temperature. The sealed tube was then placed in a preheated oil bath at $100{ }^{\circ} \mathrm{C}$ for $12 \mathrm{~h}$. After completion of the reaction, the mixture was cooled and diluted with dichloromethane $(10 \mathrm{~mL})$. The mixture was filtered through a Celite and silica gel pad and was washed with dichloromethane $(3 \times 10 \mathrm{~mL})$. The reaction mass was poured into $15 \mathrm{~mL}$ of water and shaken well. The organic layer was separated, dried on anhydrous sodium sulphate bed and concentrated under vacuum. The residue was purified by silica gel column chromatography using suitable eluent to afford the desired pure product 4. 
Procedure for the synthesis of ortho-alkynyl benzamides with triisopropyl silyl acetylenes (5ao, 5bo, 5io, 5go, and 5jo)
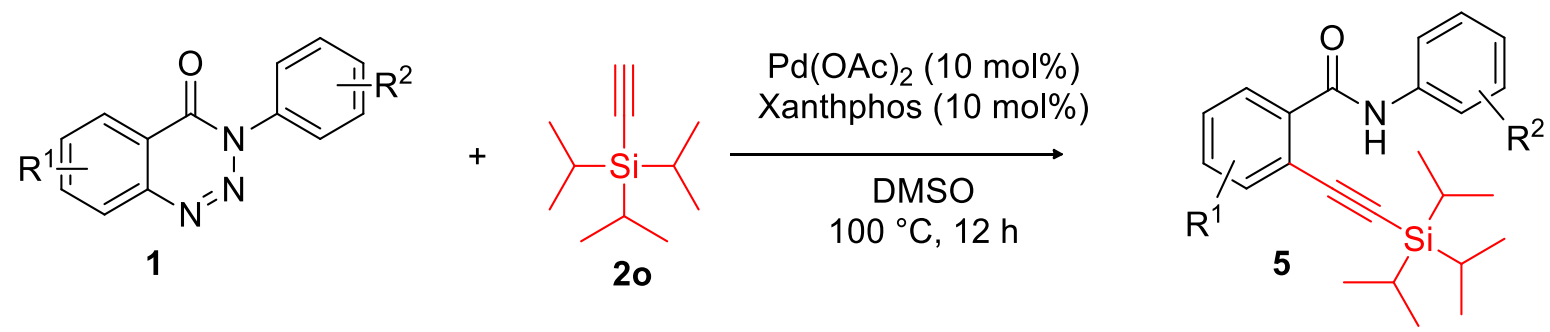

A sealed tube containing 1,2,3-benzotriazin-4(3H)-ones $1(0.40 \mathrm{mmol})$, triisopropyl acetylene $20(0.60 \mathrm{mmol}), \mathrm{Pd}(\mathrm{OAc})_{2}(0.04 \mathrm{mmol})$ and Xantphos $(0.04 \mathrm{mmol})$ was evacuated and purged with Argon gas three times at room temperature. To that mixture and anhydrous DMSO $(2 \mathrm{~mL})$ was added and stirred for 2 minutes at room temperature. The sealed tube was then placed in a preheated oil bath at $100{ }^{\circ} \mathrm{C}$ for $12 \mathrm{~h}$. After completion of the reaction, the mixture was cooled and diluted with dichloromethane $(10 \mathrm{~mL})$. The mixture was filtered through a Celite and silica gel pad and was washed with dichloromethane $(3 \times 10 \mathrm{~mL})$. The reaction mass was poured into $15 \mathrm{~mL}$ of water and shaken well. The organic layer was separated, dried on anhydrous sodium sulphate bed and concentrated under vacuum. The residue was purified by silica gel column chromatography using suitable eluent to afford the desired pure product $\mathbf{5}$.

\section{Procedure for the synthesis of ortho-alkynyl benzamides with terminal alkyl alkynes} (5ak, 5al, 5am, and 5an)

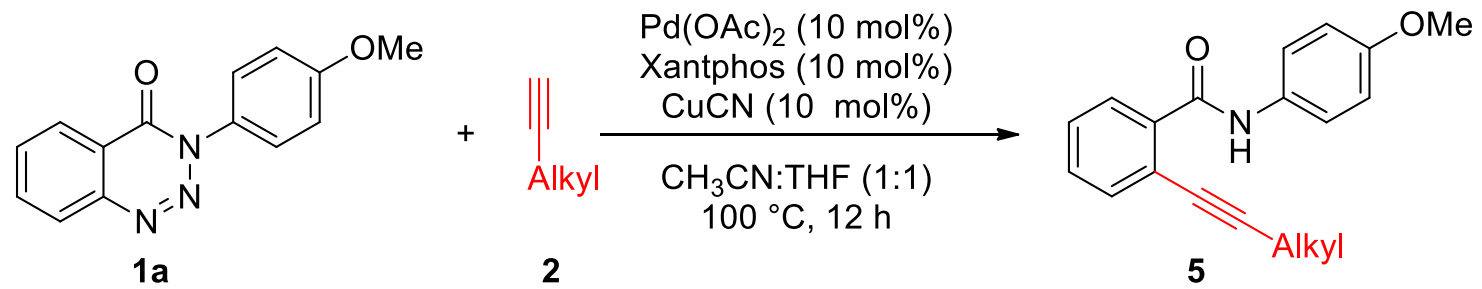

A sealed tube containing 1,2,3-Benzotriazin-4(3H)-ones 1a $(0.40 \mathrm{mmol})$, alkyl acetylene $2(0.60 \mathrm{mmol}), \mathrm{Pd}(\mathrm{OAc})_{2}(0.04 \mathrm{mmol})$, Xantphos $(0.04 \mathrm{mmol})$, and $\mathrm{CuCN}(0.04$ $\mathrm{mmol}$ ) was evacuated and purged with Argon gas three times at room temperature. To that mixture and anhydrous $\mathrm{CH}_{3} \mathrm{CN}$ :THF (1:1) $(2 \mathrm{~mL})$ was added and stirred for 2 minutes at room temperature. The sealed tube was then placed in a preheated oil bath at $100{ }^{\circ} \mathrm{C}$ for $12 \mathrm{~h}$. After completion of the reaction, the mixture was cooled and diluted with dichloromethane $(10 \mathrm{~mL})$. The mixture was filtered through a Celite and silica gel pad and was washed with dichloromethane $(3 \times 10 \mathrm{~mL})$. The reaction mass was poured into $15 \mathrm{~mL}$ of water and shaken well. The organic layer was separated, dried on anhydrous sodium sulphate bed and concentrated under vacuum. The residue was purified by silica gel column chromatography using suitable eluent to afford the desired pure product 5 . 
General procedure for the one pot synthesis of $(Z)$-3-benzylideneisobenzofuran-1(3H)one (6)<smiles>COc1ccc(-n2nnc3ccccc3c2=O)cc1</smiles>

1a (i) $\mathrm{Pd}(\mathrm{OAc})_{2}(10 \mathrm{~mol} \%)$

Xantphos (10 mol\%) $\mathrm{CuCN}(10 \mathrm{~mol} \%)$ $\mathrm{CH}_{3} \mathrm{CN}: \mathrm{THF}(1: 1)$ 100 ? $\mathrm{C}, 12 \mathrm{~h}$

(ii) $10 \%$ aq. $\mathrm{HCl}$ $50{ }^{\circ} \mathrm{C}, 3 \mathrm{~h}$

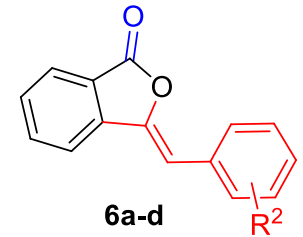

6a, $R^{2}=H(81 \%)$

6b, $R^{2}=4-$ Pentyl $(85 \%)$

6c, $R^{2}=4-\mathrm{OMe}(88 \%)$

6d, $R^{2}=3$-Methyl $(79 \%)$

A sealed tube containing 3-(4-methoxyphenyl)benzo[d][1,2,3]triazin-4(3H)-one 1a (101 mg, $0.40 \mathrm{mmol})$, terminal alkyne 2 (0.60 mmol), $\mathrm{Pd}(\mathrm{OAc})_{2}(9 \mathrm{mg}, 0.04 \mathrm{mmol})$, Xantphos (7 mg, $0.04 \mathrm{mmol}$ ), and $\mathrm{CuCN}$ ( $4 \mathrm{mg}, 0.04 \mathrm{mmol}$ ) was evacuated and purged with Argon gas three times at room temperature. To this mixture), dry $\mathrm{CH}_{3} \mathrm{CN}$ :THF (1:1) $(2 \mathrm{~mL})$ was added and stirred for 2 minutes at room temperature. The seal tube was then placed in a preheated oil bath at $100{ }^{\circ} \mathrm{C}$ for $12 \mathrm{~h} .10 \%$ aq. $\mathrm{HCl}(5 \mathrm{~mL})$ was charged to the reaction mass and stirred at $50{ }^{\circ} \mathrm{C}$ for $2 \mathrm{~h}$. The reaction mass was neutralized with $2 \% \mathrm{NaOH}$ solution and extracted with dichloromethane $(10 \mathrm{~mL})$ like 3 times. The organic layer was separated, dried on anhydrous sodium sulphate bed and concentrated under vacuum. The residue was purified by silica gel column chromatography with suitable eluent to afford the product 6 .

\section{General procedure for the synthesis of 3-benzylated isoindolinones (7).}



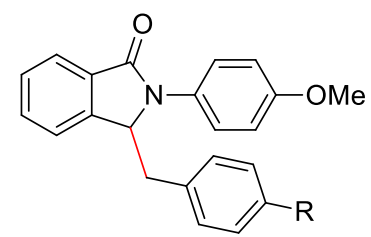

7a: $\mathrm{R}=\mathrm{H}$

7b: $R=$ Methyl

3-Benzyl-2-(4-methoxyphenyl)isoindolin-1-one (7) has been prepared by following the literature procedure. ${ }^{4}$ To a well dried reaction tube containing $3(0.40 \mathrm{mmol}), \mathrm{Pd} / \mathrm{C} 10 \% \mathrm{wt}$ loading $(0.04 \mathrm{mmol})$, and acetic acid $(1 \mathrm{~mL})$ was charged. The reaction tube was evacuated and refilled with hydrogen gas and stirred at room temperature for $2 \mathrm{~h}$. After completion of the reaction, the reaction mass was diluted with $10 \mathrm{~mL}$ water and extracted with dichloromethane $(3 \times 10 \mathrm{~mL})$. The organic layers were combined and dried in anhydrous sodium sulphate bed and concentrated. The crude product was purified by flash chromatography with a suitable eluent to afford 7.

Procedure for the synthesis ortho-alkylated benzamides (8).

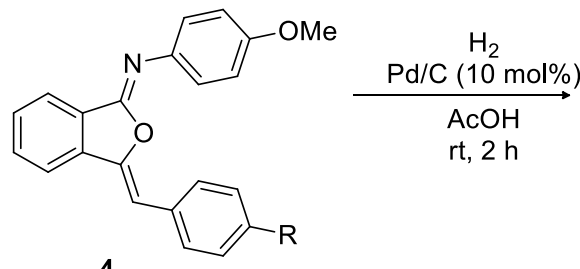

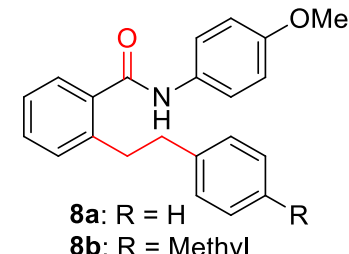

8b: $R=$ Methy 
The hydrogenation of (Z)-3-benzylidene(imino)isobenzofuranones (4) has carried out by following the literature procedure. ${ }^{4}$ To a well dried reaction tube containing 4 (164 mg, $0.5 \mathrm{mmol}), \mathrm{Pd} / \mathrm{C} 10 \%$ wt loading $(0.05 \mathrm{mmol}, 5 \mathrm{mg})$, and acetic acid $(1 \mathrm{~mL})$ were charged. The reaction tube was evacuated and refilled with hydrogen gas and stirred for 2 hours at rt. After completion of the reaction, the reaction mass was diluted with $10 \mathrm{~mL}$ water and extracted with dichloromethane $(3 \times 10 \mathrm{~mL})$. The organic layers were combined and dried in a sodium sulphate bed and concentrated. The crude product was purified by flash chromatography with a suitable eluent to afford $\mathbf{8}$.

Table-1. Optimization of reaction conditions for ortho-alkynyl benzamide (5ak) synthesis $^{a}$

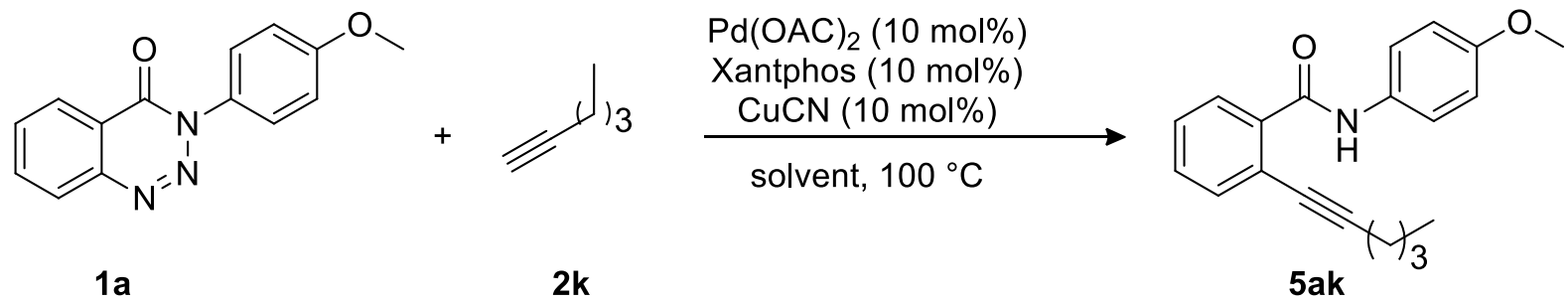

\begin{tabular}{cccc}
\hline SI No & Ligand & Solvent & Yield (\%) $^{c}$ \\
\hline 1. & Xantphos & $\mathrm{DMSO}$ & 15 \\
2 & Xantphos & $\mathrm{CH}_{3} \mathrm{CN}$ & 52 \\
$3^{a}$ & $\mathrm{PPh}_{3}$ & $\mathrm{CH}_{3} \mathrm{CN}$ & 43 \\
4 & dppm & $\mathrm{CH}_{3} \mathrm{CN}$ & 35 \\
5 & dppp & $\mathrm{CH}_{3} \mathrm{CN}$ & 48 \\
6 & $2,2^{\prime}-$ bipyridine & $\mathrm{CH}$ CN & - \\
7 & Xantphos & $\mathrm{CH}_{3} \mathrm{CN}: \mathrm{DMF}(1: 1)$ & 51 \\
8 & Xantphos & $\mathrm{CH}_{3} \mathrm{CN}: \mathrm{DMA}(1: 1)$ & 48 \\
9 & Xantphos & $\mathrm{CH}_{3} \mathrm{CN}: \mathrm{NMP}(1: 1)$ & 42 \\
10 & Xantphos & $\mathrm{CH}_{3} \mathrm{CN}$ :toluene $(1: 1)$ & 36 \\
11 & Xantphos & $\mathrm{CH}_{3} \mathrm{CN} / \mathrm{THF}(1: 1)$ & $89(85)^{d}$
\end{tabular}

${ }^{a}$ Conditions: 1a $(0.4 \mathrm{mmol}), 2 \mathbf{k}(0.60 \mathrm{mmol}), \mathrm{Pd}(\mathrm{OAc})_{2}(0.04 \mathrm{mmol})$, Ligand $(0.04 \mathrm{mmol})$, $\mathrm{CuCN}(0.04 \mathrm{mmol})$, dry solvent $(2 \mathrm{~mL})$ and carried out under argon atmosphere. ${ }^{b} 0.08 \mathrm{mmol}$ of ligand has used. ${ }^{c} \mathrm{GC}$ yields. ${ }^{d}$ Isolated yield

\section{References}

1. Miura, T.; Morimoto, M.; Yamauchi, M.; Murakami, M. J. Org. Chem. 2010, 75, 5359-5362.

2. Thorat, V. H.; Upadhyay, N. S.; Murakami, M.; Cheng, C. H. Adv. Synth. Catal. 2018, 360, 284-289

3. Brahmchari, D.; Akhilesh, A. K.; Mehta, S. J. Org. Chem., 2018, 83, 3339-3347.

4. Couty, S.; Liegault, B.; Meyer, C.; Cossy, J. Tetrahedron. 2006, 62, 3882-3895. 


\section{Spectral data of ${ }^{1} \mathrm{H},{ }^{13} \mathrm{C}$ NMR and HRMS}

(Z)-3-Benzylidene-2-(4-methoxyphenyl)isoindolin-1-one (3aa)

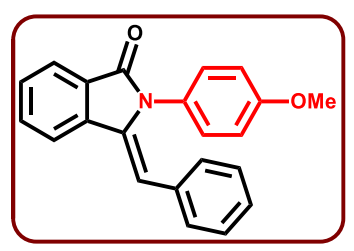

White solid (120 mg, 92\% yield); mp: $165-166{ }^{\circ} \mathrm{C} ;{ }^{1} \mathrm{H}$ NMR $\left(500 \mathrm{MHz}, \mathrm{CDCl}_{3}\right) \delta 7.95(\mathrm{~d}, J$ $=7.5 \mathrm{~Hz}, 1 \mathrm{H}), 7.85(\mathrm{~d}, J=7.7 \mathrm{~Hz}, 1 \mathrm{H}), 7.67$ (t, $J=7.4 \mathrm{~Hz}, 1 \mathrm{H}), 7.55$ (t, $J=7.4 \mathrm{~Hz}, 1 \mathrm{H}), 6.96$ $(\mathrm{dd}, J=16.7,7.8 \mathrm{~Hz}, 5 \mathrm{H}), 6.87(\mathrm{~d}, J=7.2 \mathrm{~Hz}, 2 \mathrm{H}), 6.82(\mathrm{~s}, 1 \mathrm{H}), 6.62(\mathrm{~d}, J=8.4 \mathrm{~Hz}, 2 \mathrm{H})$, $3.71(\mathrm{~s}, 3 \mathrm{H}) ;{ }^{13} \mathrm{C}$ NMR $\left(126 \mathrm{MHz}, \mathrm{CDCl}_{3}\right) \delta 168.1,158.3,138.5,134.6,133.5,132.3,129.1$, 128.7, 128.3, 127.8, 127.1, 126.5, 123.8, 119.3, 113.6, 107.4, 55.5; HRMS calculated for $\mathrm{C}_{22} \mathrm{H}_{17} \mathrm{NO}_{2}[\mathrm{M}+\mathrm{H}]^{+}:$328.1338, found 328.1336; Purification flash chromatography; Eluent: hexane/EtOAc $=85 / 15$.

\section{(Z)-3-Benzylidene-2-(p-tolyl)isoindolin-1-one (3ba)}

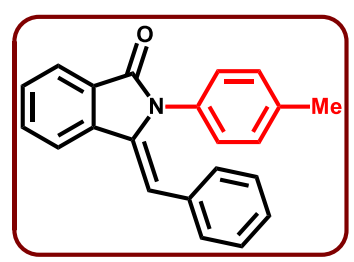

White solid (113 mg, 91\% yield); mp: $145-146{ }^{\circ} \mathrm{C} ;{ }^{1} \mathrm{H}$ NMR $\left(500 \mathrm{MHz}, \mathrm{CDCl}_{3}\right) \delta 7.95(\mathrm{~d}, J$ $=7.6 \mathrm{~Hz}, 1 \mathrm{H}), 7.85(\mathrm{~d}, J=7.8 \mathrm{~Hz}, 1 \mathrm{H}), 7.67(\mathrm{~d}, J=0.6 \mathrm{~Hz}, 1 \mathrm{H}), 7.59-7.50(\mathrm{~m}, 1 \mathrm{H}), 6.99-$ $6.85(\mathrm{~m}, 9 \mathrm{H}), 6.81(\mathrm{~s}, 1 \mathrm{H}), 2.22(\mathrm{~s}, 3 \mathrm{H}) ;{ }^{13} \mathrm{C} \mathrm{NMR}\left(126 \mathrm{MHz}, \mathrm{CDCl}_{3}\right) \delta 168.0,138.6,136.6$, $134.5,133.6,133.2,132.3,129.1,129.1,128.7$, 127.8, 127.1, 126.7, 126.4, 123.8, 119.3, 107.5, 20.9; HRMS calculated for $\mathrm{C}_{22} \mathrm{H}_{17} \mathrm{NO}[\mathrm{M}+\mathrm{H}]^{+}$: 312.1388, found 312.1381; Purification flash chromatography; Eluent: hexane/EtOAc $=87 / 13$.

(Z)-3-Benzylidene-2-(4-chlorophenyl)isoindolin-1-one (3ca)



White solid (113 mg, 85\% yield); mp: $142-143{ }^{\circ} \mathrm{C} ;{ }^{1} \mathrm{H}$ NMR $\left(500 \mathrm{MHz}, \mathrm{CDCl}_{3}\right) \delta 7.94(\mathrm{~d}, J=$ $7.6 \mathrm{~Hz}, 1 \mathrm{H}), 7.86(\mathrm{~d}, J=7.8 \mathrm{~Hz}, 1 \mathrm{H}), 7.68(\mathrm{td}, J=7.6,1.1 \mathrm{~Hz}, 1 \mathrm{H}), 7.56(\mathrm{td}, J=7.5,0.7 \mathrm{~Hz}$, $1 \mathrm{H}), 7.04(\mathrm{tt}, J=4.3,2.1 \mathrm{~Hz}, 3 \mathrm{H}), 7.01-6.95(\mathrm{~m}, 4 \mathrm{H}), 6.89-6.83(\mathrm{~m}, 3 \mathrm{H}) ;{ }^{13} \mathrm{C} \mathrm{NMR}(126$ 
$\left.\mathrm{MHz}, \mathrm{CDCl}_{3}\right) \delta 167.8,138.5,134.3,134.1,133.3,132.6,132.3,129.3,129.1,128.3,128.2$, $127.5,127.3,126.9,123.9,119.4,107.7$; HRMS calculated for $\mathrm{C}_{21} \mathrm{H}_{14} \mathrm{ClNO}[\mathrm{M}+\mathrm{H}]^{+}$: 332.0842, found 332.0832; Purification flash chromatography; Eluent: hexane/EtOAc = $87 / 13$.

(Z)-3-benzylidene-2-(3,4,5-trimethoxyphenyl)isoindolin-1-one (3da)

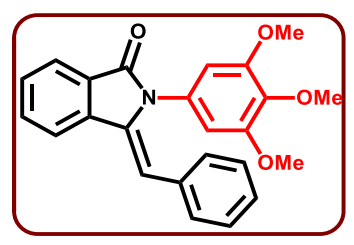

White solid (113 mg, 73\% yield); mp: $189-190{ }^{\circ} \mathrm{C} ;{ }^{1} \mathrm{H}$ NMR $\left(500 \mathrm{MHz}, \mathrm{CDCl}_{3}\right) \delta 7.95(\mathrm{~d}, J$ $=7.4 \mathrm{~Hz}, 1 \mathrm{H}), 7.86(\mathrm{~d}, J=7.6 \mathrm{~Hz}, 1 \mathrm{H}), 7.68(\mathrm{t}, J=7.4 \mathrm{~Hz}, 1 \mathrm{H}), 7.56(\mathrm{t}, J=7.4 \mathrm{~Hz}, 1 \mathrm{H}), 7.07$ - $6.97(\mathrm{~m}, 3 \mathrm{H}), 6.91(\mathrm{~d}, J=6.8 \mathrm{~Hz}, 2 \mathrm{H}), 6.85(\mathrm{~s}, 1 \mathrm{H}), 6.33(\mathrm{~s}, 2 \mathrm{H}), 3.74(\mathrm{~s}, 3 \mathrm{H}), 3.62(\mathrm{~s}, 6 \mathrm{H})$; ${ }^{13} \mathrm{C} \mathrm{NMR}\left(126 \mathrm{MHz}, \mathrm{CDCl}_{3}\right) \delta 167.8,152.7,138.4,136.9,134.3,133.9,132.5,131.2,129.2$, 128.7, 127.6, 127.1, 126.9, 123.8, 119.3, 107.6, 105.3, 60.8, 55.9; HRMS calculated for $\mathrm{C}_{24} \mathrm{H}_{21} \mathrm{NO}_{4}[\mathrm{M}+\mathrm{H}]^{+}:$388.1549, found 388.1547; Purification flash chromatography; Eluent: hexane $/ \mathrm{EtOAc}=75 / 25$.

(Z)-3-Benzylidene-2-phenylisoindolin-1-one (3ea)

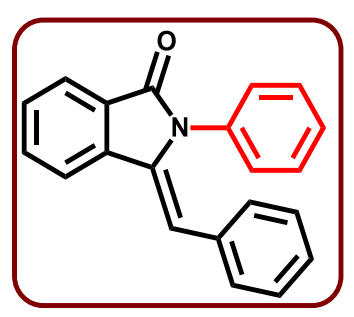

White solid (113 mg, 95\% yield); mp: $199-200{ }^{\circ} \mathrm{C}{ }^{1} \mathrm{H}$ NMR $\left(500 \mathrm{MHz}, \mathrm{CDCl}_{3}\right) \delta 7.96(\mathrm{~d}, J=$ $7.6 \mathrm{~Hz}, 1 \mathrm{H}), 7.86$ (d, $J=7.8 \mathrm{~Hz}, 1 \mathrm{H}), 7.68(\mathrm{~s}, 1 \mathrm{H}), 7.56$ (d, J=7.5 Hz, 1H), $7.12-7.04$ (m, $5 \mathrm{H}), 6.96(\mathrm{~d}, J=7.1 \mathrm{~Hz}, 1 \mathrm{H}), 6.92(\mathrm{dd}, J=8.1,6.7 \mathrm{~Hz}, 2 \mathrm{H}), 6.88-6.82(\mathrm{~m}, 3 \mathrm{H}) ;{ }^{13} \mathrm{C} \mathrm{NMR}$ $\left(126 \mathrm{MHz}, \mathrm{CDCl}_{3}\right) \delta 168.0,138.6,135.8,134.3,133.5,132.4,129.2,129.1,128.1,127.8$, 127.2, 126.7, 126.5, 123.9, 119.4, 107.6; HRMS calculated for $\mathrm{C}_{21} \mathrm{H}_{15} \mathrm{NO}[\mathrm{M}+\mathrm{H}]^{+}: 298.1232$, found 298.1224; Purification flash chromatography; Eluent: hexane/EtOAc = 90/10. 


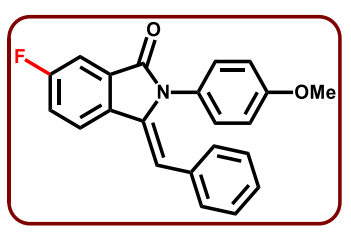

White solid (112 mg, 81\% yield); mp: $189-190{ }^{\circ} \mathrm{C} ;{ }^{1} \mathrm{H}$ NMR $\left(500 \mathrm{MHz}, \mathrm{CDCl}_{3}\right) \delta 7.82(\mathrm{dd}, J$ $=8.5,4.3 \mathrm{~Hz}, 1 \mathrm{H}), 7.60(\mathrm{dd}, J=7.4,2.4 \mathrm{~Hz}, 1 \mathrm{H}), 7.37(\mathrm{td}, J=8.7,2.4 \mathrm{~Hz}, 1 \mathrm{H}), 7.02-6.92$ $(\mathrm{m}, 5 \mathrm{H}), 6.85(\mathrm{~d}, J=7.3 \mathrm{~Hz}, 2 \mathrm{H}), 6.76(\mathrm{~s}, 1 \mathrm{H}), 6.64-6.59(\mathrm{~m}, 2 \mathrm{H}), 3.71(\mathrm{~s}, 3 \mathrm{H}) ;{ }^{13} \mathrm{C} \mathrm{NMR}$ $\left(126 \mathrm{MHz}, \mathrm{CDCl}_{3}\right) \delta 166.9\left(\mathrm{~d}, J_{\mathrm{C}-\mathrm{F}}=3.4 \mathrm{~Hz}\right), 163.4\left(\mathrm{~d}, J_{\mathrm{C}-\mathrm{F}}=249.5 \mathrm{~Hz}\right), 158.3,134.3\left(\mathrm{~d}, J_{\mathrm{C}-\mathrm{F}}\right.$ $=2.3 \mathrm{~Hz}), 133.8,133.2,129.6\left(\mathrm{~d}, J_{\mathrm{C}-\mathrm{F}}=8.9 \mathrm{~Hz}\right), 129.1,128.4,128.2,127.1,126.5,121.2(\mathrm{~d}$, $\left.J_{\mathrm{C}-\mathrm{F}}=8.5 \mathrm{~Hz}\right), 120.0\left(\mathrm{~d}, J_{\mathrm{C}-\mathrm{F}}=24.2 \mathrm{~Hz}\right), 113.6,110.1(\mathrm{~d}, J=23.7 \mathrm{~Hz}), 107.6,55.4$; HRMS calculated for $\mathrm{C}_{22} \mathrm{H}_{16} \mathrm{FNO}_{2}[\mathrm{M}+\mathrm{H}]^{+}:$346.1243, found 346.1247; Purification flash chromatography; Eluent: hexane/EtOAc $=93 / 07$.

(Z)-3-Benzylidene-2-(4-methoxyphenyl)-6-methylisoindolin-1-one (3ga)

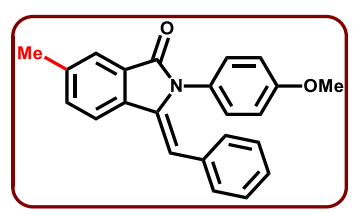

White solid (130 mg, 95\% yield); mp: $179-180{ }^{\circ} \mathrm{C} ;{ }^{1} \mathrm{H}$ NMR $\left(500 \mathrm{MHz}, \mathrm{CDCl}_{3}\right) \delta 7.73$ (s, 1H), $7.72(\mathrm{~d}, J=8.0 \mathrm{~Hz}, 1 \mathrm{H}), 7.47$ (d, $J=7.9 \mathrm{~Hz}, 1 \mathrm{H}), 7.00-6.90(\mathrm{~m}, 5 \mathrm{H}), 6.85$ (d, $J=7.3$ $\mathrm{Hz}, 2 \mathrm{H}), 6.75(\mathrm{~s}, 1 \mathrm{H}), 6.63-6.58(\mathrm{~m}, 2 \mathrm{H}), 3.70(\mathrm{~s}, 3 \mathrm{H}), 2.50(\mathrm{~s}, 3 \mathrm{H}) ;{ }^{13} \mathrm{C} \mathrm{NMR}(126 \mathrm{MHz}$, $\left.\mathrm{CDCl}_{3}\right) \delta 168.2,158.2,139.5,136.1,134.7,133.6,133.4,129.1,128.8,128.3,128.0,127.9$, 127.1, 126.3, 123.8, 119.1, 114.4, 113.5, 106.7, 55.4, 21.5; HRMS calculated for $\mathrm{C}_{23} \mathrm{H}_{19} \mathrm{NO}_{2}$ $[\mathrm{M}+\mathrm{H}]^{+}:$342.1494, found 342.1490; Purification flash chromatography; Eluent: hexane $/$ EtOAc $=85 / 15$.

(Z)-methyl 3-benzylidene-1-oxo-2-phenylisoindoline-5-carboxylate (3ha)



White solid (119 mg, 84\% yield); mp: $171-172{ }^{\circ} \mathrm{C}$; ${ }^{1} \mathrm{H}$ NMR (500 $\left.\mathrm{MHz}, \mathrm{CDCl}_{3}\right) \delta 8.55$ (s, 1H), $8.21(\mathrm{~d}, J=7.9 \mathrm{~Hz}, 1 \mathrm{H}), 8.01(\mathrm{~d}, J=7.9 \mathrm{~Hz}, 1 \mathrm{H}), 7.09$ (s, 5H), 6.99 (t, $J=7.1 \mathrm{~Hz}, 1 \mathrm{H})$, $6.95-6.89(\mathrm{~m}, 3 \mathrm{H}), 6.86(\mathrm{~d}, J=7.4 \mathrm{~Hz}, 2 \mathrm{H}), 4.01(\mathrm{~s}, 3 \mathrm{H}) ;{ }^{13} \mathrm{C} \mathrm{NMR}\left(126 \mathrm{MHz}, \mathrm{CDCl}_{3}\right) \delta$ 
$166.9,166.3,138.5,135.5,133.7,133.5,133.0,131.0,130.1,129.1,128.2,127.2,127.0$, $126.9,126.8,123.8,121.0,108.9,52.6$; HRMS calculated for $\mathrm{C}_{23} \mathrm{H}_{17} \mathrm{NO}_{3}[\mathrm{M}+\mathrm{H}]^{+}: 356.1287$, found 356.1280; Purification flash chromatography; Eluent: hexane/EtOAc $=80 / 20$.

(Z)-2-(4-Methoxyphenyl)-3-(4-methylbenzylidene)isoindolin-1-one (3ab)

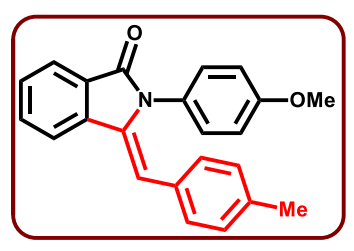

White solid (121 mg, 89\% yield); mp: $169-170{ }^{\circ} \mathrm{C} ;{ }^{1} \mathrm{H}$ NMR $\left(500 \mathrm{MHz}, \mathrm{CDCl}_{3}\right) \delta 7.94(\mathrm{~d}, J$ $=7.6 \mathrm{~Hz}, 1 \mathrm{H}), 7.84(\mathrm{~d}, J=7.8 \mathrm{~Hz}, 1 \mathrm{H}), 7.68-7.63(\mathrm{~m}, 1 \mathrm{H}), 7.56-7.50(\mathrm{~m}, 1 \mathrm{H}), 7.01-6.95$ $(\mathrm{m}, 2 \mathrm{H}), 6.79(\mathrm{~s}, 1 \mathrm{H}), 6.77-6.73(\mathrm{~m}, 4 \mathrm{H}), 6.66-6.61(\mathrm{~m}, 2 \mathrm{H}), 3.72(\mathrm{~s}, 3 \mathrm{H}), 2.21(\mathrm{~s}, 3 \mathrm{H})$; ${ }^{13} \mathrm{C}$ NMR $\left(126 \mathrm{MHz}, \mathrm{CDCl}_{3}\right) \delta 168.1,158.2,138.6,136.3,134.0,132.2,130.5,129.1,128.9$, $128.8,128.2,127.8,127.7,123.7,119.2,113.5,107.6,55.4,21.1$; HRMS calculated for $\mathrm{C}_{23} \mathrm{H}_{19} \mathrm{NO}_{2}[\mathrm{M}+\mathrm{H}]^{+}:$342.1494, found 342.1483; Purification flash chromatography; Eluent: hexane $/ \mathrm{EtOAc}=85 / 15$.

(Z)-3-(4-Ethylbenzylidene)-2-(4-methoxyphenyl)isoindolin-1-one (3ac)



White solid (106 mg, 75\% yield); mp: 161-162 ${ }^{\circ} \mathrm{C} ;{ }^{1} \mathrm{H}$ NMR (500 MHz, $\left.\mathrm{CDCl}_{3}\right) \delta 7.94(\mathrm{~d}, J$ $=7.6 \mathrm{~Hz}, 1 \mathrm{H}), 7.84(\mathrm{~d}, J=7.8 \mathrm{~Hz}, 1 \mathrm{H}), 7.66(\mathrm{dd}, J=11.0,4.1 \mathrm{~Hz}, 1 \mathrm{H}), 7.53(\mathrm{t}, J=7.5 \mathrm{~Hz}$, 1H), $7.00-6.92(\mathrm{~m}, 2 \mathrm{H}), 6.81(\mathrm{~s}, 1 \mathrm{H}), 6.77(\mathrm{~s}, 4 \mathrm{H}), 6.68-6.55(\mathrm{~m}, 2 \mathrm{H}), 3.70(\mathrm{~s}, 3 \mathrm{H}), 2.49$ $(\mathrm{q}, J=7.6 \mathrm{~Hz}, 2 \mathrm{H}), 1.13(\mathrm{t}, J=7.6 \mathrm{~Hz}, 3 \mathrm{H}) ;{ }^{13} \mathrm{C} \mathrm{NMR}\left(126 \mathrm{MHz}, \mathrm{CDCl}_{3}\right) \delta 168.0,158.2$, $142.9,138.5,134.2,132.2,130.7,129.1,128.9,128.7,128.3,127.7,126.6,123.7,119.2$, 113.4, 107.6, 28.5, 15.7; HRMS calculated for $\mathrm{C}_{24} \mathrm{H}_{21} \mathrm{NO}_{2}[\mathrm{M}+\mathrm{H}]^{+}$: 356.1651, found 356.1650; Purification flash chromatography; Eluent: hexane/EtOAc $=85 / 15$.

(Z)-2-(4-Methoxyphenyl)-3-(4-pentylbenzylidene)isoindolin-1-one (3ad)

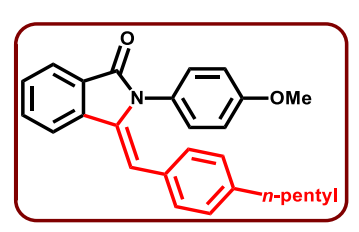


White solid (119 mg, 75\% yield); mp: $158-159{ }^{\circ} \mathrm{C} ;{ }^{1} \mathrm{H}$ NMR (500 MHz, $\left.\mathrm{CDCl}_{3}\right) \delta 7.93$ (d, $J$ $=7.5 \mathrm{~Hz}, 1 \mathrm{H}), 7.80(\mathrm{~d}, J=7.8 \mathrm{~Hz}, 1 \mathrm{H}), 7.62(\mathrm{t}, J=7.5 \mathrm{~Hz}, 1 \mathrm{H}), 7.51(\mathrm{t}, J=7.5 \mathrm{~Hz}, 1 \mathrm{H}), 7.01$ - $6.95(\mathrm{~m}, 2 \mathrm{H}), 6.80(\mathrm{~s}, 1 \mathrm{H}), 6.76(\mathrm{~d}, J=7.6 \mathrm{~Hz}, 4 \mathrm{H}), 6.62(\mathrm{t}, J=6.0 \mathrm{~Hz}, 2 \mathrm{H}), 3.69(\mathrm{~s}, 3 \mathrm{H})$, $2.49-2.42(\mathrm{~m}, 2 \mathrm{H}), 1.54-1.48(\mathrm{~m}, 2 \mathrm{H}), 1.36(\mathrm{dd}, J=14.7,7.3 \mathrm{~Hz}, 2 \mathrm{H}), 1.30-1.26(\mathrm{~m}$, 2H), $0.93(\mathrm{t}, J=7.2 \mathrm{~Hz}, 3 \mathrm{H}) ;{ }^{13} \mathrm{C}$ NMR $\left(126 \mathrm{MHz}, \mathrm{CDCl}_{3}\right) \delta 167.8,158.0,141.3,138.4$, $133.9,132.0,130.6,128.9,128.7,128.6,128.1,127.6,127.0,123.4,119.1,113.2,107.5$, 55.1, 35.4, 31.2, 31.1, 22.3, 13.8; HRMS calculated for $\mathrm{C}_{27} \mathrm{H}_{27} \mathrm{NO}_{2}[\mathrm{M}+\mathrm{H}]^{+}: 398.2120$, found 398.2113; Purification flash chromatography; Eluent: hexane/EtOAc $=85 / 15$.

\section{(Z)-3-(4-(Tert-butyl)benzylidene)-2-(4-methoxyphenyl)isoindolin-1-one (3ae)}

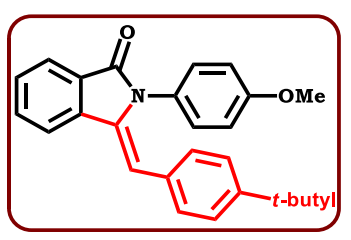

White solid (133 mg, 87\% yield); mp: 146-147 ${ }^{\circ} \mathrm{C} ;{ }^{1} \mathrm{H}$ NMR $\left(500 \mathrm{MHz}, \mathrm{CDCl}_{3}\right) \delta 7.94(\mathrm{~d}, J$ $=7.5 \mathrm{~Hz}, 1 \mathrm{H}), 7.85(\mathrm{~d}, J=7.7 \mathrm{~Hz}, 1 \mathrm{H}), 7.66(\mathrm{t}, J=7.4 \mathrm{~Hz}, 1 \mathrm{H}), 7.54$ (t, $J=7.3 \mathrm{~Hz}, 1 \mathrm{H}), 6.94$ (t, $J=7.8 \mathrm{~Hz}, 4 \mathrm{H}), 6.82(\mathrm{~s}, 1 \mathrm{H}), 6.77(\mathrm{~d}, J=7.6 \mathrm{~Hz}, 2 \mathrm{H}), 6.58(\mathrm{~d}, J=8.0 \mathrm{~Hz}, 2 \mathrm{H}), 3.69$ (s, $3 \mathrm{H}), 1.22(\mathrm{~s}, 9 \mathrm{H}) ;{ }^{13} \mathrm{C} \mathrm{NMR}\left(126 \mathrm{MHz}, \mathrm{CDCl}_{3}\right) \delta 168.0,158.2,149.7,138.5,134.5,132.2$, 130.6, 129.0, 128.7, 128.4, 127.9, 123.9, 123.7, 119.3, 113.3, 107.5, 55.2, 34.3, 31.1; HRMS calculated for $\mathrm{C}_{26} \mathrm{H}_{25} \mathrm{NO}_{2}[\mathrm{M}+\mathrm{H}]^{+}:$384.1964, found 384.1959; Purification flash chromatography; Eluent: hexane/EtOAc $=85 / 15$.

(Z)-3-(4-Methoxybenzylidene)-2-(4-methoxyphenyl)isoindolin-1-one (3af)



White solid (111 mg, 78\% yield); mp: $170-171{ }^{\circ} \mathrm{C} ;{ }^{1} \mathrm{H}$ NMR $\left(500 \mathrm{MHz}, \mathrm{CDCl}_{3}\right) \delta 7.93(\mathrm{~d}, J$ $=7.6 \mathrm{~Hz}, 1 \mathrm{H}), 7.82(\mathrm{~d}, J=7.8 \mathrm{~Hz}, 1 \mathrm{H}), 7.67-7.61(\mathrm{~m}, 1 \mathrm{H}), 7.52(\mathrm{t}, J=7.5 \mathrm{~Hz}, 1 \mathrm{H}), 7.02-$ $6.98(\mathrm{~m}, 2 \mathrm{H}), 6.79(\mathrm{~d}, J=8.5 \mathrm{~Hz}, 2 \mathrm{H}), 6.77(\mathrm{~d}, J=3.1 \mathrm{~Hz}, 1 \mathrm{H}), 6.68-6.63(\mathrm{~m}, 2 \mathrm{H}), 6.52-$ $6.46(\mathrm{~m}, 2 \mathrm{H}), 3.72(\mathrm{~s}, 3 \mathrm{H}), 3.70(\mathrm{~s}, 3 \mathrm{H}) ;{ }^{13} \mathrm{C} \mathrm{NMR}\left(126 \mathrm{MHz}, \mathrm{CDCl}_{3}\right) \delta 168.1,158.4,158.3$, $138.7,133.5,132.2$, 130.6, 128.9, 128.9, 128.3, 127.7, 126.0, 123.8, 119.2, 113.6, 112.8, 107.5, 55.5, 55.2; HRMS calculated for $\mathrm{C}_{23} \mathrm{H}_{19} \mathrm{NO}_{3}[\mathrm{M}+\mathrm{H}]^{+}:$358.1443, found 358.1445; Purification flash chromatography; Eluent: hexane/EtOAc $=85 / 15$. 


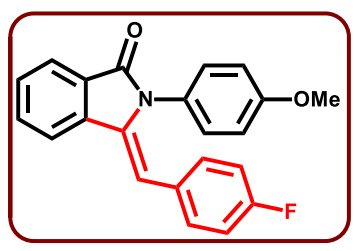

White solid (121 mg, 88\% yield); mp: 205-206 ${ }^{\circ} \mathrm{C} ;{ }^{1} \mathrm{H}$ NMR (500 MHz, $\left.\mathrm{CDCl}_{3}\right) \delta 7.94(\mathrm{~d}, J$ $=7.6 \mathrm{~Hz}, 1 \mathrm{H}), 7.83(\mathrm{~d}, J=7.8 \mathrm{~Hz}, 1 \mathrm{H}), 7.67(\mathrm{td}, J=7.6,1.1 \mathrm{~Hz}, 1 \mathrm{H}), 7.55(\mathrm{td}, J=7.5,0.7$ $\mathrm{Hz}, 1 \mathrm{H}), 6.99-6.94(\mathrm{~m}, 2 \mathrm{H}), 6.83(\mathrm{ddd}, J=8.3,5.0,2.2 \mathrm{~Hz}, 2 \mathrm{H}), 6.75$ (s, 1H), 6.65 (ddd, $J=$ 8.6, 5.3, $2.1 \mathrm{~Hz}, 4 \mathrm{H}), 3.73(\mathrm{~s}, 3 \mathrm{H}) ;{ }^{13} \mathrm{C} \mathrm{NMR}\left(126 \mathrm{MHz}, \mathrm{CDCl}_{3}\right) \delta 168.0,161.3\left(\mathrm{~d}, J_{\mathrm{C}-\mathrm{F}}=\right.$ $247.4 \mathrm{~Hz}), 158.4,138.4,134.7,132.3,130.7\left(\mathrm{~d}, J_{\mathrm{C}-\mathrm{F}}=8.1 \mathrm{~Hz}\right), 129.6\left(\mathrm{~d}, J_{\mathrm{C}-\mathrm{F}}=3.3 \mathrm{~Hz}\right)$, 129.2 , 128.5, 128.3, 127.8, 123.8, 119.3, $114.1\left(\mathrm{~d}, J_{\mathrm{C}-\mathrm{F}}=21.6 \mathrm{~Hz}\right), 113.6,106.0,55.5$; HRMS calculated for $\mathrm{C}_{22} \mathrm{H}_{16} \mathrm{FNO}_{2}[\mathrm{M}+\mathrm{H}]^{+}:$346.1243, found 346.1239; Purification flash chromatography; Eluent: hexane/EtOAc $=85 / 15$.

(Z)-2-(4-Methoxyphenyl)-3-(3-methylbenzylidene)isoindolin-1-one (3ah)

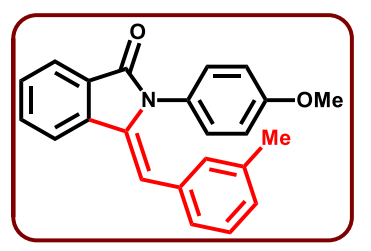

White solid (108 mg, 79\% yield); mp: $112-113{ }^{\circ} \mathrm{C} ;{ }^{1} \mathrm{H}$ NMR $\left(500 \mathrm{MHz}, \mathrm{CDCl}_{3}\right) \delta 7.94(\mathrm{~d}, J=$ $7.4 \mathrm{~Hz}, 1 \mathrm{H}), 7.84(\mathrm{~d}, J=7.6 \mathrm{~Hz}, 1 \mathrm{H}), 7.66$ (t, $J=7.4 \mathrm{~Hz}, 1 \mathrm{H}), 7.54$ (t, $J=7.3 \mathrm{~Hz}, 1 \mathrm{H}), 6.99$ (d, $J=$ $8.0 \mathrm{~Hz}, 2 \mathrm{H}), 6.91(\mathrm{t}, J=7.4 \mathrm{~Hz}, 1 \mathrm{H}), 6.79$ (dd, $J=15.2,7.5 \mathrm{~Hz}, 3 \mathrm{H}), 6.64$ (d, $J=8.0 \mathrm{~Hz}, 2 \mathrm{H}), 6.58$ (s, $1 \mathrm{H}), 3.72(\mathrm{~s}, 3 \mathrm{H}), 2.05(\mathrm{~s}, 3 \mathrm{H}) ;{ }^{13} \mathrm{C} \mathrm{NMR}\left(126 \mathrm{MHz}, \mathrm{CDCl}_{3}\right) \delta$ 168.1, 158.2, 138.6, 136.7, $134.4,133.3,132.2,130.4,129.0,128.9,128.3,127.8,127.4,127.2,126.1,123.7,119.3$, 113.5, 107.6, 55.4, 20.9; HRMS calculated for $\mathrm{C}_{23} \mathrm{H}_{19} \mathrm{NO}_{2}[\mathrm{M}+\mathrm{H}]^{+}$: 342.1494, found 342.1478; Purification flash chromatography; Eluent: hexane/EtOAc $=85 / 15$.

((Z)-3-(3-Fluorobenzylidene)-2-(4-methoxyphenyl)isoindolin-1-one) (3ai)



White solid (108 mg, 78\% yield); mp: $142-143{ }^{\circ} \mathrm{C} ;{ }^{1} \mathrm{H}$ NMR (500 MHz, $\left.\mathrm{CDCl}_{3}\right) \delta 7.94(\mathrm{~d}, J$ $=7.5 \mathrm{~Hz}, 1 \mathrm{H}), 7.83(\mathrm{~d}, J=7.8 \mathrm{~Hz}, 1 \mathrm{H}), 7.67(\mathrm{td}, J=7.6,1.1 \mathrm{~Hz}, 1 \mathrm{H}), 7.56(\mathrm{td}, J=7.5,0.7$ 
$\mathrm{Hz}, 1 \mathrm{H}), 7.01-6.96(\mathrm{~m}, 2 \mathrm{H}), 6.91(\mathrm{td}, J=8.0,6.1 \mathrm{~Hz}, 1 \mathrm{H}), 6.73(\mathrm{~s}, 1 \mathrm{H}), 6.72-6.68(\mathrm{~m}$, $1 \mathrm{H}), 6.68-6.64(\mathrm{~m}, 3 \mathrm{H}), 6.55(\mathrm{~d}, J=10.0 \mathrm{~Hz}, 1 \mathrm{H}), 3.73(\mathrm{~s}, 3 \mathrm{H}) ;{ }^{13} \mathrm{C}$ NMR $(126 \mathrm{MHz}$, $\left.\mathrm{CDCl}_{3}\right) \delta 168.0,161.8(\mathrm{~d}, J=245.3 \mathrm{~Hz}), 158.5,138.2,135.8(\mathrm{~d}, J=8.1 \mathrm{~Hz}), 135.6,132.4$, 129.4, 128.6 (d, $J=4.8 \mathrm{~Hz}), 128.5,128.3,127.8,125.0$ (d, $J=2.7 \mathrm{~Hz}), 123.8,119.4,116.0$ $(\mathrm{d}, J=22.1 \mathrm{~Hz}), 113.7,113.4(\mathrm{~d}, J=21.2 \mathrm{~Hz}), 105.6,55.5$; HRMS calculated for $\mathrm{C}_{22} \mathrm{H}_{16} \mathrm{FNO}_{2}[\mathrm{M}+\mathrm{H}]^{+}:$346.1243, found 346.1236; Purification flash chromatography; Eluent: hexane/EtOAc $=85 / 15$.

\section{(Z)-2-(4-Methoxyphenyl)-3-(pyridin-2-ylmethylene)isoindolin-1-one (3aj)}

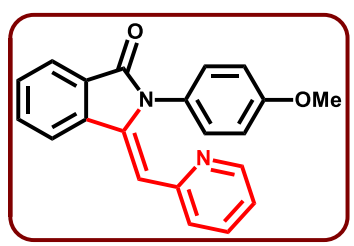

White solid (93 mg, 71\% yield); mp: $168-169{ }^{\circ} \mathrm{C} ;{ }^{1} \mathrm{H}$ NMR $\left(500 \mathrm{MHz}, \mathrm{CDCl}_{3}\right) \delta 8.57-8.14$ $(\mathrm{m}, 1 \mathrm{H}), 7.94(\mathrm{~d}, J=7.5 \mathrm{~Hz}, 1 \mathrm{H}), 7.87(\mathrm{~d}, J=7.7 \mathrm{~Hz}, 1 \mathrm{H}), 7.68(\mathrm{td}, J=7.6,0.9 \mathrm{~Hz}, 1 \mathrm{H})$, $7.57(\mathrm{t}, J=7.5 \mathrm{~Hz}, 1 \mathrm{H}), 7.15(\mathrm{~s}, 1 \mathrm{H}), 7.01(\mathrm{~d}, J=8.7 \mathrm{~Hz}, 2 \mathrm{H}), 6.83$ (t, $J=39.4 \mathrm{~Hz}, 3 \mathrm{H}), 6.63$ $(\mathrm{d}, J=8.7 \mathrm{~Hz}, 2 \mathrm{H}), 3.72(\mathrm{~s}, 3 \mathrm{H}) ;{ }^{13} \mathrm{C}$ NMR $\left(126 \mathrm{MHz}, \mathrm{CDCl}_{3}\right) \delta 168.1,158.3,152.7,138.3$, $137.1,134.5,134.5,134.5,132.6,129.7,129.1,128.1,127.9,123.8,119.7,113.7,55.5$; HRMS calculated for $\mathrm{C}_{21} \mathrm{H}_{16} \mathrm{~N}_{2} \mathrm{O}_{2}[\mathrm{M}+\mathrm{H}]^{+}$: 329.1290, found 329.1282; Purification flash chromatography; Eluent:hexane/EtOAc $=80 / 20$.

\section{(Z)-N-((Z)-3-benzylideneisobenzofuran-1(3H)-ylidene)-4-methoxyaniline (4aa)}

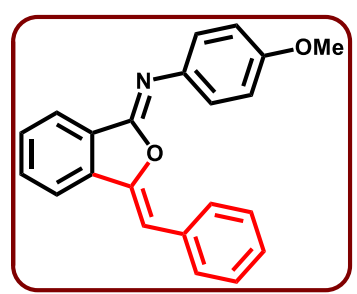

Pale yellow solid (111 mg, 85\% yield); mp: 132-133 ${ }^{\circ} \mathrm{C} ;{ }^{1} \mathrm{H}$ NMR (500 MHz, $\left.\mathrm{CDCl}_{3}\right) \delta 7.99$ (d, $J=7.6 \mathrm{~Hz}, 1 \mathrm{H}), 7.77$ (d, $J=7.7 \mathrm{~Hz}, 2 \mathrm{H}), 7.69$ (d, $J=7.7 \mathrm{~Hz}, 1 \mathrm{H}), 7.57$ (dd, $J=17.4,8.1$ $\mathrm{Hz}, 3 \mathrm{H}), 7.51(\mathrm{t}, J=7.4 \mathrm{~Hz}, 1 \mathrm{H}), 7.36(\mathrm{t}, J=7.6 \mathrm{~Hz}, 2 \mathrm{H}), 7.25(\mathrm{t}, J=7.3 \mathrm{~Hz}, 1 \mathrm{H}), 6.97(\mathrm{~d}, J$ $=8.7 \mathrm{~Hz}, 2 \mathrm{H}), 6.25(\mathrm{~s}, 1 \mathrm{H}), 3.87(\mathrm{~s}, 3 \mathrm{H}) ;{ }^{13} \mathrm{C} \mathrm{NMR}\left(126 \mathrm{MHz}, \mathrm{CDCl}_{3}\right) \delta 157.1,153.0,148.2$, $138.5,137.3$, 134.0, 131.9, 129.6, 129.1, 128.6, 127.4, 125.5, 123.6, 119.5, 113.9, 102.9, 55.5; HRMS calculated for $\mathrm{C}_{22} \mathrm{H}_{17} \mathrm{NO}_{2}[\mathrm{M}+\mathrm{H}]^{+}: 328.1338$, found 328.1334; Purification flash chromatography; Eluent:hexane/EtOAc = 95/05. 


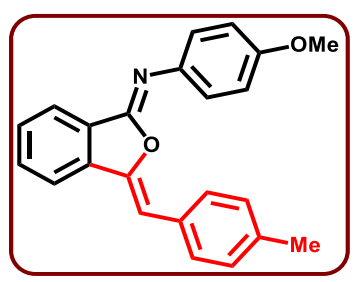

Yellow solid (110 mg, 81\% yield); mp: $128-129{ }^{\circ} \mathrm{C} ;{ }^{1} \mathrm{H}$ NMR $\left(500 \mathrm{MHz}, \mathrm{CDCl}_{3}\right) \delta 7.99(\mathrm{~d}, J$ $=7.7 \mathrm{~Hz}, 1 \mathrm{H}), 7.68(\mathrm{~d}, J=8.0 \mathrm{~Hz}, 3 \mathrm{H}), 7.57(\mathrm{ddd}, J=6.7,6.1,1.3 \mathrm{~Hz}, 3 \mathrm{H}), 7.52-7.46(\mathrm{~m}$, 1H), $7.18(\mathrm{~d}, J=8.0 \mathrm{~Hz}, 2 \mathrm{H}), 7.01-6.96(\mathrm{~m}, 2 \mathrm{H}), 6.23$ (s, 1H), 3.88 (s, 3H), 2.37 (s, 3H); ${ }^{13} \mathrm{C}$ NMR $\left(126 \mathrm{MHz}, \mathrm{CDCl}_{3}\right) \delta 157.0,153.1,147.6,138.6,137.4,137.4,131.8,131.2,129.5$, 129.4, 129.0, 125.5, 123.5, 119.3, 113.9, 103.0, 55.4, 21.3; HRMS calculated for $\mathrm{C}_{23} \mathrm{H}_{19} \mathrm{NO}_{2}$ $[\mathrm{M}+\mathrm{H}]^{+}: \quad 342.1494$, found 342.1487; Purification flash chromatography; Eluent: hexane $/$ EtOAc $=95 / 05$.

$(Z)-N-((Z)-3-(4-E t h y l b e n z y l i d e n e) i s o b e n z o f u r a n-1(3 H)$-ylidene)-4-methoxyaniline (4ac)

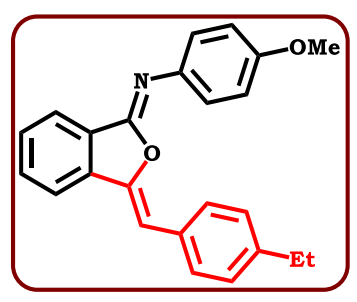

Yellow solid (114 mg, 80\% yield); mp: $113-114{ }^{\circ} \mathrm{C} ;{ }^{1} \mathrm{H}$ NMR $\left(500 \mathrm{MHz}, \mathrm{CDCl}_{3}\right) \delta 8.03-$ $7.96(\mathrm{~m}, 1 \mathrm{H}), 7.72-7.68(\mathrm{~m}, 3 \mathrm{H}), 7.57$ (ddd, $J=9.0,7.1,1.5 \mathrm{~Hz}, 3 \mathrm{H}), 7.50$ (t, $J=7.5 \mathrm{~Hz}$, 1H), $7.20(\mathrm{~d}, J=8.1 \mathrm{~Hz}, 2 \mathrm{H}), 7.00-6.97(\mathrm{~m}, 2 \mathrm{H}), 6.25(\mathrm{~s}, 1 \mathrm{H}), 3.88(\mathrm{~s}, 3 \mathrm{H}), 2.66(\mathrm{q}, J=7.6$ $\mathrm{Hz}, 2 \mathrm{H}), 1.26(\mathrm{t}, J=7.6 \mathrm{~Hz}, 3 \mathrm{H}) ;{ }^{13} \mathrm{C} \mathrm{NMR}\left(126 \mathrm{MHz}, \mathrm{CDCl}_{3}\right) \delta 157.0,153.2,147.6,143.8$, $138.6,137.4,131.9,131.4,129.5,129.4,129.1,128.2,125.5,123.5,119.4,113.9,103.0$, 55.5, 28.7, 15.4; HRMS calculated for $\mathrm{C}_{24} \mathrm{H}_{21} \mathrm{NO}_{2}[\mathrm{M}+\mathrm{H}]^{+}$: 356.1651, found 356.1644; Purification flash chromatography; Eluent: hexane/EtOAc $=95 / 05$.

(Z)-4-Methoxy- $N$-((Z)-3-(4-pentylbenzylidene)isobenzofuran-1(3H)-ylidene)aniline (4ad)

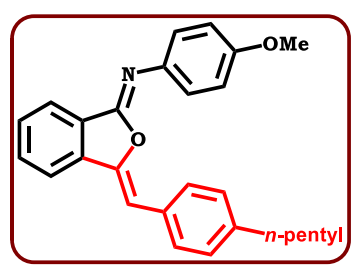


Pale yellow solid (119 mg, 75\% yield); mp: 108-109 ${ }^{\circ} \mathrm{C} ;{ }^{1} \mathrm{H}$ NMR (500 MHz, $\left.\mathrm{CDCl}_{3}\right) \delta 7.99$ $(\mathrm{d}, J=7.5 \mathrm{~Hz}, 1 \mathrm{H}), 7.69$ (d, $J=7.5 \mathrm{~Hz}, 3 \mathrm{H}), 7.57$ (dd, $J=16.0,7.7 \mathrm{~Hz}, 3 \mathrm{H}), 7.50(\mathrm{t}, J=7.2$ Hz, 1H), 7.18 (d, $J=7.5 \mathrm{~Hz}, 2 \mathrm{H}), 6.98$ (d, $J=7.7 \mathrm{~Hz}, 2 \mathrm{H}), 6.24$ (s, 1H), 3.87 (d, $J=9.8 \mathrm{~Hz}$, $3 \mathrm{H}), 2.61(\mathrm{t}, J=7.3 \mathrm{~Hz}, 2 \mathrm{H}), 1.64(\mathrm{~s}, 2 \mathrm{H}), 1.34(\mathrm{~s}, 4 \mathrm{H}), 0.90(\mathrm{~d}, J=6.2 \mathrm{~Hz}, 3 \mathrm{H}),{ }^{13} \mathrm{C} \mathrm{NMR}$ $\left(126 \mathrm{MHz}, \mathrm{CDCl}_{3}\right) \delta 157.0,153.1,147.6,142.6,138.7,137.4,131.8,131.4,129.5,129.4$, $129.0,128.7,125.5,123.5,119.4,113.9,103.0,55.5,35.7,31.5,31.1,22.5,14.0$; HRMS calculated for $\mathrm{C}_{27} \mathrm{H}_{27} \mathrm{NO}_{2}[\mathrm{M}+\mathrm{H}]^{+}:$398.2120, found 398.2117; Purification flash chromatography; Eluent: hexane/EtOAc $=95 / 05$.

(Z)-N-((Z)-3-(4-(Tert-butyl)benzylidene)isobenzofuran-1(3H)-ylidene)-4-methoxyaniline (4ae)

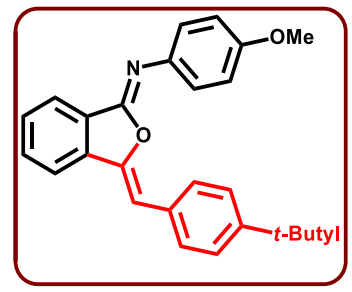

Pale brown solid (127 mg, 83\% yield); mp: $132-133{ }^{\circ} \mathrm{C} ;{ }^{1} \mathrm{H}$ NMR $\left(500 \mathrm{MHz}, \mathrm{CDCl}_{3}\right) \delta 8.00$ $(\mathrm{d}, J=7.7 \mathrm{~Hz}, 1 \mathrm{H}), 7.71(\mathrm{dd}, J=10.2,8.1 \mathrm{~Hz}, 3 \mathrm{H}), 7.61-7.55(\mathrm{~m}, 3 \mathrm{H}), 7.51(\mathrm{t}, J=7.5 \mathrm{~Hz}$, $1 \mathrm{H}), 7.41-7.36(\mathrm{~m}, 2 \mathrm{H}), 7.02-6.97(\mathrm{~m}, 2 \mathrm{H}), 6.26(\mathrm{~s}, 1 \mathrm{H}), 3.89(\mathrm{~s}, 3 \mathrm{H}), 1.35(\mathrm{~s}, 9 \mathrm{H}) ;{ }^{13} \mathrm{C}$ NMR $\left(126 \mathrm{MHz}, \mathrm{CDCl}_{3}\right) \delta 157.0,153.2,150.7,147.7,138.6,137.4,131.9,131.2,129.5$, 129.4, 128.9, 125.6, 125.5, 123.5, 119.4, 113.9, 102.9, 55.5, 34.6, 31.2; HRMS calculated for $\mathrm{C}_{26} \mathrm{H}_{25} \mathrm{NO}_{2}[\mathrm{M}+\mathrm{H}]^{+}:$384.1964, found 384.1957; Purification flash chromatography; Eluent: hexane $/ \mathrm{EtOAc}=95 / 05$.

(Z)-4-Methoxy- $N$-((Z)-3-(4-methoxybenzylidene)isobenzofuran-1(3H)-ylidene)aniline (4af)

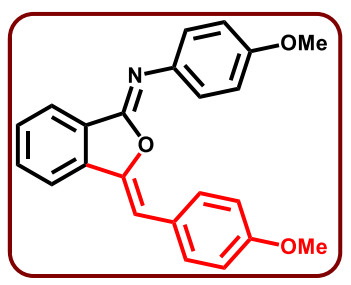

Yellow solid (120 mg, 84\% yield); mp: $182-183{ }^{\circ} \mathrm{C} ;{ }^{1} \mathrm{H}$ NMR $\left(500 \mathrm{MHz}, \mathrm{CDCl}_{3}\right) \delta 7.98(\mathrm{~d}, J$ $=7.6 \mathrm{~Hz}, 1 \mathrm{H}), 7.71(\mathrm{~d}, J=7.7 \mathrm{~Hz}, 2 \mathrm{H}), 7.65(\mathrm{~d}, J=7.4 \mathrm{~Hz}, 1 \mathrm{H}), 7.56(\mathrm{t}, J=7.8 \mathrm{~Hz}, 3 \mathrm{H})$, 7.47 (t, $J=7.2 \mathrm{~Hz}, 1 \mathrm{H}), 6.98(\mathrm{~d}, J=8.4 \mathrm{~Hz}, 2 \mathrm{H}), 6.89$ (d, $J=7.7 \mathrm{~Hz}, 2 \mathrm{H}), 6.20$ (s, 1H), 3.87 (s, 3H), $3.84(\mathrm{~s}, 3 \mathrm{H}) ;{ }^{13} \mathrm{C}$ NMR $\left(126 \mathrm{MHz}, \mathrm{CDCl}_{3}\right) \delta 159.0,156.9,153.3,146.7,138.6,137.4$, 
$131.8,130.5,129.2,129.1,126.7,125.4,123.5,119.2,114.1,113.9,102.7,55.4,55.3$; HRMS calculated for $\mathrm{C}_{23} \mathrm{H}_{19} \mathrm{NO}_{3}[\mathrm{M}+\mathrm{H}]^{+}:$358.1443, found 358.1437; Purification flash chromatography; Eluent: hexane/EtOAc $=95 / 05$.

$(Z)-N-((Z)-3-(4-F l u o r o b e n z y l i d e n e) i s o b e n z o f u r a n-1(3 H)$-ylidene)-4-methoxyaniline (4ag)

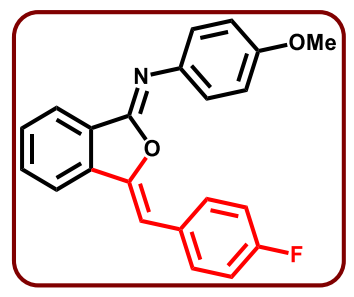

Pale yellow solid (102 mg, 74\% yield); mp: 157-158 ${ }^{\circ} \mathrm{C} ;{ }^{1} \mathrm{H}$ NMR (500 MHz, $\left.\mathrm{CDCl}_{3}\right) \delta 8.00$ (d, $J=7.5 \mathrm{~Hz}, 1 \mathrm{H}), 7.75(\mathrm{t}, J=5.8 \mathrm{~Hz}, 2 \mathrm{H}), 7.69(\mathrm{~d}, J=7.6 \mathrm{~Hz}, 1 \mathrm{H}), 7.60$ (t, $J=7.3 \mathrm{~Hz}, 1 \mathrm{H})$, $7.52(\mathrm{~d}, J=7.7 \mathrm{~Hz}, 3 \mathrm{H}), 7.05(\mathrm{t}, J=8.2 \mathrm{~Hz}, 2 \mathrm{H}), 6.97(\mathrm{~d}, J=8.0 \mathrm{~Hz}, 2 \mathrm{H}), 6.22(\mathrm{~s}, 1 \mathrm{H}), 3.88$ $(\mathrm{s}, 3 \mathrm{H}) ;{ }^{13} \mathrm{C} \mathrm{NMR}\left(126 \mathrm{MHz}, \mathrm{CDCl}_{3}\right) \delta 161.9(\mathrm{~d}, J=248.6 \mathrm{~Hz}), 157.1,152.9,147.8(\mathrm{~d}, J=$ $2.6 \mathrm{~Hz}), 138.5,137.2,131.9,130.7$ (d, $J=7.9 \mathrm{~Hz}), 130.2$ (d, $J=3.4 \mathrm{~Hz}), 129.6,129.5,125.4$, 123.6, 119.4, $115.6(\mathrm{~d}, J=21.5 \mathrm{~Hz}), 113.9,101.7,55.5$; HRMS calculated for $\mathrm{C}_{22} \mathrm{H}_{16} \mathrm{FNO}_{2}$ $[\mathrm{M}+\mathrm{H}]^{+}: \quad 346.1243$, found 346.1236; Purification flash chromatography; Eluent: hexane $/$ EtOAc $=95 / 05$.

(Z)-4-Methoxy- $N-((Z)-3-(3-$ methylbenzylidene)isobenzofuran-1(3H)-ylidene)aniline (4ah)

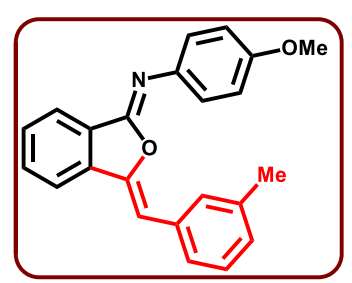

Pale yellow solid (101 mg, 74\% yield); mp: 86-87 ${ }^{\circ} \mathrm{C} ;{ }^{1} \mathrm{H}$ NMR (500 MHz, DMSO) $\delta 8.00$ $(\mathrm{d}, J=7.7 \mathrm{~Hz}, 1 \mathrm{H}), 7.76$ (s, 1H), 7.69 (d, $J=7.8 \mathrm{~Hz}, 1 \mathrm{H}), 7.58$ (dd, $J=13.6,8.0 \mathrm{~Hz}, 3 \mathrm{H})$, $7.51(\mathrm{t}, J=7.4 \mathrm{~Hz}, 1 \mathrm{H}), 7.45$ (d, $J=7.7 \mathrm{~Hz}, 1 \mathrm{H}), 7.25$ (d, $J=8.9 \mathrm{~Hz}, 1 \mathrm{H}), 7.08$ (d, $J=7.5$ $\mathrm{Hz}, 1 \mathrm{H}), 6.97(\mathrm{~d}, J=8.7 \mathrm{~Hz}, 2 \mathrm{H}), 6.23(\mathrm{~s}, 1 \mathrm{H}), 3.86(\mathrm{~s}, 3 \mathrm{H}), 2.37(\mathrm{~s}, 3 \mathrm{H}) ;{ }^{13} \mathrm{C} \mathrm{NMR}(126$ $\left.\mathrm{MHz}, \mathrm{CDCl}_{3}\right) \delta 157.0,153.1,148.1,138.6,138.2,137.4,133.9,131.9,129.6,129.5,128.5$, $128.3,126.4,125.5,123.5,119.5,113.9,103.0,55.4,21.5$; HRMS calculated for $\mathrm{C}_{23} \mathrm{H}_{19} \mathrm{NO}_{2}$ $[\mathrm{M}+\mathrm{H}]^{+}: \quad 342.1494$, found 342.1485; Purification flash chromatography; Eluent: hexane $/ \mathrm{EtOAc}=95 / 05$. 


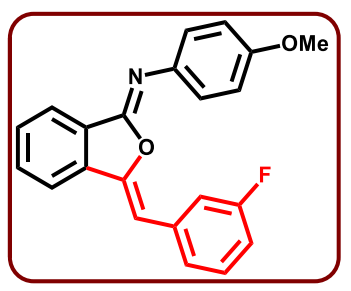

Pale yellow solid (103 mg, 75\% yield); mp: 92-93 ${ }^{\circ} \mathrm{C} ;{ }^{1} \mathrm{H}$ NMR (500 MHz, DMSO) $\delta 8.01$ (d, $J=7.6 \mathrm{~Hz}, 1 \mathrm{H}), 7.70(\mathrm{~d}, J=7.7 \mathrm{~Hz}, 1 \mathrm{H}), 7.61$ (t, $J=7.6 \mathrm{~Hz}, 2 \mathrm{H}), 7.54$ (dd, $J=7.7,5.3 \mathrm{~Hz}$, 3H), $7.43(\mathrm{~d}, J=7.7 \mathrm{~Hz}, 1 \mathrm{H}), 7.31(\mathrm{dd}, J=14.3,7.7 \mathrm{~Hz}, 1 \mathrm{H}), 7.01-6.93(\mathrm{~m}, 3 \mathrm{H}), 6.22$ (s, $1 \mathrm{H}), 3.87(\mathrm{~s}, 3 \mathrm{H}) ;{ }^{13} \mathrm{C} \mathrm{NMR}\left(126 \mathrm{MHz}, \mathrm{CDCl}_{3}\right) \delta 162.9\left(\mathrm{~d}, J_{\mathrm{C}-\mathrm{F}}=244.8 \mathrm{~Hz}\right), 157.3,152.5$, 149.0, 138.3, 136.9, $136.1\left(\mathrm{~d}, J_{\mathrm{C}-\mathrm{F}}=8.6 \mathrm{~Hz}\right), 132.0,129.9$ - $129.7(\mathrm{~m}), 129.8,129.8,125.4$, $124.8\left(\mathrm{~d}, J_{\mathrm{C}-\mathrm{F}}=2.7 \mathrm{~Hz}\right), 123.6,119.6,115.4\left(\mathrm{~d}, J_{\mathrm{C}-\mathrm{F}}=23.0 \mathrm{~Hz}\right), 114.2\left(\mathrm{~d}, J_{\mathrm{C}-\mathrm{F}}=21.4 \mathrm{~Hz}\right)$, 114.0, $101.6\left(\mathrm{~d}, J_{\mathrm{C}-\mathrm{F}}=2.8 \mathrm{~Hz}\right), 55.5$; HRMS calculated for $\mathrm{C}_{22} \mathrm{H}_{16} \mathrm{FNO}_{2}[\mathrm{M}+\mathrm{H}]^{+}: 346.1243$, found 346.1231; Purification flash chromatography; Eluent: hexane/EtOAc $=95 / 05$ (Z)-4-Methoxy- $N$-((Z)-3-(thiophen-3-ylmethylene)isobenzofuran-1(3H)-ylidene)aniline (4ap)

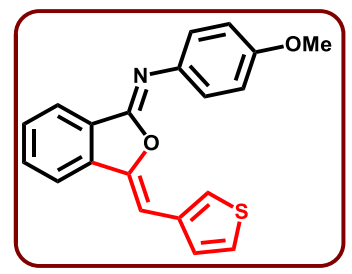

Dark brown solid (97 mg, 73\% yield); mp: 101-102 ${ }^{\circ} \mathrm{C} ;{ }^{1} \mathrm{H}$ NMR (500 MHz, $\left.\mathrm{CDCl}_{3}\right) \delta 7.98$ (d, $J=7.7 \mathrm{~Hz}, 1 \mathrm{H}), 7.65(\mathrm{~d}, J=7.7 \mathrm{~Hz}, 1 \mathrm{H}), 7.61-7.54(\mathrm{~m}, 2 \mathrm{H}), 7.51-7.47$ (m, 3H), 7.45 $(\mathrm{d}, J=4.9 \mathrm{~Hz}, 1 \mathrm{H}), 7.33-7.28(\mathrm{~m}, 1 \mathrm{H}), 6.97(\mathrm{~d}, J=8.7 \mathrm{~Hz}, 2 \mathrm{H}), 6.34(\mathrm{~s}, 1 \mathrm{H}), 3.87(\mathrm{~s}, 3 \mathrm{H})$; ${ }^{13} \mathrm{C} \mathrm{NMR}\left(126 \mathrm{MHz}, \mathrm{CDCl}_{3}\right) \delta 156.9,153.1,147.3,138.7,137.0,134.9,131.9,129.6,129.4$, $128.3,125.5,125.1,124.1,123.6,119.3,114.0$, 97.5, 55.46; HRMS calculated for $\mathrm{C}_{20} \mathrm{H}_{15} \mathrm{NO}_{2} \mathrm{~S}[\mathrm{M}+\mathrm{H}]^{+}:$334.0902, found 334.0897; Purification flash chromatography; Eluent: hexane/EtOAc $=95 / 05$.

(Z)-N-((Z)-3-Benzylidene-6-fluoroisobenzofuran-1(3H)-ylidene)-4-methoxyaniline (4fa)

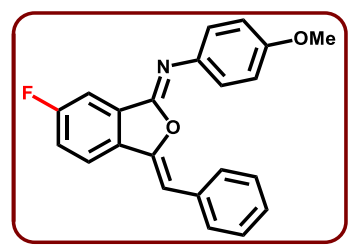


Pale yellow solid (110 mg, 80\% yield); mp: $134-135{ }^{\circ} \mathrm{C} ;{ }^{1} \mathrm{H}$ NMR $\left(500 \mathrm{MHz}, \mathrm{CDCl}_{3}\right) \delta 7.76$ $(\mathrm{d}, J=7.5 \mathrm{~Hz}, 2 \mathrm{H}), 7.66$ (t, $J=8.9 \mathrm{~Hz}, 2 \mathrm{H}), 7.57$ (d, $J=7.9 \mathrm{~Hz}, 2 \mathrm{H}), 7.37$ (t, $J=7.3 \mathrm{~Hz}, 2 \mathrm{H})$, 7.29 (dd, $J=19.3,9.4 \mathrm{~Hz}, 2 \mathrm{H}), 6.97$ (d, $J=7.9 \mathrm{~Hz}, 2 \mathrm{H}), 6.20$ (s, 1H), 3.87 (s, 3H); ${ }^{13} \mathrm{C} \mathrm{NMR}$ $\left(126 \mathrm{MHz} \mathrm{CDCl}_{3}\right) \delta 163.6(\mathrm{~d}, J=250.2 \mathrm{~Hz}), 157.3,151.6(\mathrm{~d}, J=4.8 \mathrm{~Hz}), 147.5,138.0$, $133.8,133.2(\mathrm{~d}, J=2.1 \mathrm{~Hz}), 131.8(\mathrm{~d}, J=9.8 \mathrm{~Hz}), 129.0,128.7,127.5,125.7,121.3(\mathrm{~d}, J=$ $9.0 \mathrm{~Hz}), 120.1(\mathrm{~d}, J=24.7 \mathrm{~Hz}), 114.0,110.0(\mathrm{~d}, J=24.5 \mathrm{~Hz}), 102.7,55.5$; HRMS calculated for $\mathrm{C}_{22} \mathrm{H}_{16} \mathrm{FNO}_{2}[\mathrm{M}+\mathrm{H}]^{+}:$346.1243, found 346.1238; Purification flash chromatography; Eluent: hexane/EtOAc $=95 / 05$.

\section{(Z)- $N$-((Z)-3-Benzylidene-6-methylisobenzofuran-1(3H)-ylidene)-4-methoxyaniline}

\section{(4ga)}

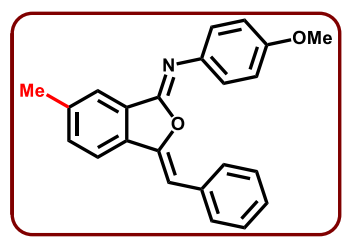

Pale yellow solid (112 mg, 82\% yield); mp: 87-88 ${ }^{\circ} \mathrm{C} ;{ }^{1} \mathrm{H}$ NMR (500 MHz, $\left.\mathrm{CDCl}_{3}\right) \delta 7.80$ (d, $J=0.6 \mathrm{~Hz}, 1 \mathrm{H}), 7.76(\mathrm{~d}, J=7.6 \mathrm{~Hz}, 2 \mathrm{H}), 7.58(\mathrm{~d}, J=7.9 \mathrm{~Hz}, 1 \mathrm{H}), 7.57-7.53(\mathrm{~m}, 2 \mathrm{H}), 7.40$ $(\mathrm{d}, J=8.0 \mathrm{~Hz}, 1 \mathrm{H}), 7.35(\mathrm{t}, J=7.7 \mathrm{~Hz}, 2 \mathrm{H}), 7.26-7.22(\mathrm{~m}, 1 \mathrm{H}), 6.99-6.95(\mathrm{~m}, 2 \mathrm{H}), 6.19$ (s, 1H), $3.87(\mathrm{~s}, 3 \mathrm{H}), 2.49(\mathrm{~s}, 3 \mathrm{H}) ;{ }^{13} \mathrm{C} \mathrm{NMR}\left(126 \mathrm{MHz}, \mathrm{CDCl}_{3}\right) \delta 157.0,153.1,148.3,140.1$, $138.6,134.9,134.2,133.2,129.9,128.9,128.6$, 127.2, 125.5, 123.5, 119.3, 113.9, 102.1, 55.4, 21.5; HRMS calculated for $\mathrm{C}_{23} \mathrm{H}_{19} \mathrm{NO}_{2}[\mathrm{M}+\mathrm{H}]^{+}:$342.1494, found 342.1486; Purification flash chromatography; Eluent: hexane/EtOAc $=93 / 07$.

(Z)- $N$-((Z)-3-Benzylidene-6-morpholinoisobenzofuran-1(3H)-ylidene)-4-methoxyaniline (4ka)

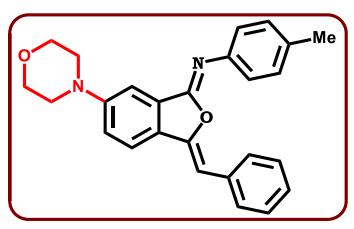

Yellow solid (135 mg, 85\% yield); mp: $172-173{ }^{\circ} \mathrm{C} ;{ }^{1} \mathrm{H}$ NMR $\left(500 \mathrm{MHz}, \mathrm{CDCl}_{3}\right) \delta 7.71(\mathrm{~d}, J$ $=7.8 \mathrm{~Hz}, 2 \mathrm{H}), 7.57(\mathrm{~d}, J=8.6 \mathrm{~Hz}, 1 \mathrm{H}), 7.43(\mathrm{~d}, J=8.1 \mathrm{~Hz}, 2 \mathrm{H}), 7.40(\mathrm{~d}, J=1.4 \mathrm{~Hz}, 1 \mathrm{H})$, $7.32(\mathrm{t}, J=7.6 \mathrm{~Hz}, 2 \mathrm{H}), 7.22(\mathrm{dd}, J=13.9,7.5 \mathrm{~Hz}, 3 \mathrm{H}), 7.17(\mathrm{dd}, J=8.6,1.7 \mathrm{~Hz}, 1 \mathrm{H}), 6.08$ $(\mathrm{s}, 1 \mathrm{H}), 3.92-3.85(\mathrm{~m}, 4 \mathrm{H}), 3.35-3.26(\mathrm{~m}, 4 \mathrm{H}), 2.40(\mathrm{~s}, 3 \mathrm{H}),{ }^{13} \mathrm{C} \mathrm{NMR}\left(126 \mathrm{MHz}, \mathrm{CDCl}_{3}\right)$ $\delta 154.0,152.6,148.3,142.9,134.5,134.4,130.9,129.3,128.9,128.7,128.5,126.8,123.9$, 
120.4, 107.9, 101.0, 66.6, 48.9, 21.1; HRMS calculated for $\mathrm{C}_{26} \mathrm{H}_{24} \mathrm{~N}_{2} \mathrm{O}_{2}[\mathrm{M}+\mathrm{H}]^{+}: 397.1916$, found 397.1914; Purification flash chromatography; Eluent:hexane/EtOAc = 80/20.

$N$-(4-Methoxyphenyl)-2-((triisopropylsilyl)ethynyl)benzamide (5ao)

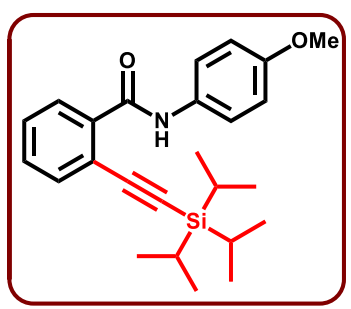

Dark brown viscous liquid (155 mg, 95\% yield); ${ }^{1} \mathrm{H}$ NMR $\left(500 \mathrm{MHz}, \mathrm{CDCl}_{3}\right) \delta 9.10(\mathrm{~s}, 1 \mathrm{H})$, $8.04-7.97(\mathrm{~m}, 1 \mathrm{H}), 7.59-7.54(\mathrm{~m}, 1 \mathrm{H}), 7.54-7.49(\mathrm{~m}, 2 \mathrm{H}), 7.43-7.36(\mathrm{~m}, 2 \mathrm{H}), 6.89-$ $6.83(\mathrm{~m}, 2 \mathrm{H}), 3.78(\mathrm{~s}, 3 \mathrm{H}), 1.06(\mathrm{~d}, J=4.0 \mathrm{~Hz}, 21 \mathrm{H}) ;{ }^{13} \mathrm{C} \mathrm{NMR}\left(126 \mathrm{MHz}, \mathrm{CDCl}_{3}\right) \delta 164.2$, $156.5,136.0,134.2,130.7,130.4,129.8,128.9,122.4,119.6,113.8,105.0,99.0,55.3,18.5$, 11.1; HRMS calculated for $\mathrm{C}_{25} \mathrm{H}_{33} \mathrm{NO}_{2} \mathrm{Si}[\mathrm{M}+\mathrm{H}]^{+}$: 408.2359, found 408.2350; Purification flash chromatography; Eluent: hexane/EtOAc $=97 / 03$.

$N$-(p-Tolyl)-2-((triisopropylsilyl)ethynyl)benzamide (5bo)

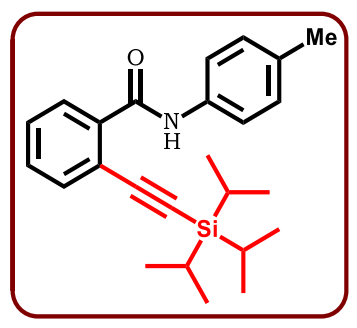

White solid; (142 mg, 91\% yield); mp: 87-88 ${ }^{\circ} \mathrm{C} ;{ }^{1} \mathrm{H}$ NMR $\left(500 \mathrm{MHz}, \mathrm{CDCl}_{3}\right) \delta 9.00(\mathrm{~s}, 1 \mathrm{H})$, $7.90-7.80(\mathrm{~m}, 1 \mathrm{H}), 7.44-7.37(\mathrm{~m}, 3 \mathrm{H}), 7.28-7.21(\mathrm{~m}, 2 \mathrm{H}), 7.00$ (d, $J=8.3 \mathrm{~Hz}, 2 \mathrm{H}), 2.19$ (s, 3H), $0.95(\mathrm{~d}, J=4.3 \mathrm{~Hz}, 21 \mathrm{H}) ;{ }^{13} \mathrm{C} \mathrm{NMR}\left(126 \mathrm{MHz}, \mathrm{CDCl}_{3}\right) \delta 136.1,135.1,134.1,133.8$, 130.2, 129.6, 129.0, 128.7, 120.5, 119.5, 104.8, 98.7, 20.7, 18.4, 11.0; HRMS calculated for $\mathrm{C}_{25} \mathrm{H}_{33} \mathrm{NOSi}[\mathrm{M}+\mathrm{H}]^{+}:$392.2410, found 392.2414; Purification flash chromatography; Eluent: hexane/EtOAc $=97 / 3$.

$N$-(4-Fluorophenyl)-2-((triisopropylsilyl)ethynyl)benzamide (5io)

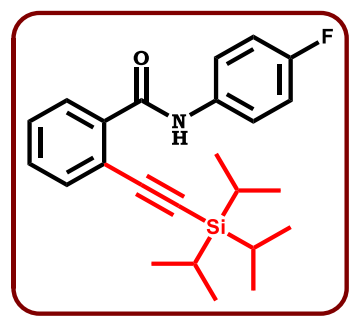


White solid; (136 mg, 86\% yield); mp: 87-88 ${ }^{\circ} \mathrm{C} ;{ }^{1} \mathrm{H}$ NMR $\left(500 \mathrm{MHz}, \mathrm{CDCl}_{3}\right) \delta 9.11(\mathrm{~s}, 1 \mathrm{H})$, $8.12-8.05(\mathrm{~m}, 1 \mathrm{H}), 7.65-7.54(\mathrm{~m}, 3 \mathrm{H}), 7.47$ (dd, $J=6.1,2.5 \mathrm{~Hz}, 2 \mathrm{H}), 7.09-7.00(\mathrm{~m}, 2 \mathrm{H})$, $1.07(\mathrm{~d}, J=4.9 \mathrm{~Hz}, 21 \mathrm{H}) ;{ }^{13} \mathrm{C} \mathrm{NMR}\left(126 \mathrm{MHz}, \mathrm{CDCl}_{3}\right) \delta 164.4,159.7\left(\mathrm{~d}, J_{\mathrm{C}-\mathrm{F}}=244.0 \mathrm{~Hz}\right)$, $135.7,134.5,133.7\left(\mathrm{~d}, J_{\mathrm{C}-\mathrm{F}}=2.8 \mathrm{~Hz}\right), 130.9,130.1,129.2,122.7\left(\mathrm{~d}, J_{\mathrm{C}-\mathrm{F}}=8.0 \mathrm{~Hz}\right), 119.8$, $115.6(\mathrm{~d}, J=22.5 \mathrm{~Hz}), 105.0,99.6,18.6,11.2$; HRMS calculated for $\mathrm{C}_{24} \mathrm{H}_{30} \mathrm{FNOSi}[\mathrm{M}+\mathrm{H}]^{+}$: 396.2159, found 396.2168; Purification flash chromatography; Eluent: hexane/EtOAc = 93/07.

\section{$\mathrm{N}$-(4-Methoxyphenyl)-5-methyl-2-((triisopropylsilyl)ethynyl)benzamide (5go)}

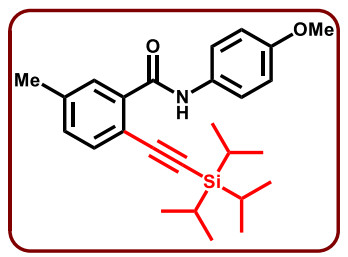

White solid; (147 mg, 87\% yield); mp: 93-94 ${ }^{\circ} \mathrm{C} ;{ }^{1} \mathrm{H}$ NMR (500 MHz, $\left.\mathrm{CDCl}_{3}\right) \delta 9.10(\mathrm{~s}, 1 \mathrm{H})$, 7.93 (s, 1H), 7.50 (t, $J=7.6 \mathrm{~Hz}, 3 \mathrm{H}), 7.25$ (d, $J=9.2 \mathrm{~Hz}, 1 \mathrm{H}), 6.89$ (d, $J=8.5 \mathrm{~Hz}, 2 \mathrm{H}), 3.82$ $(\mathrm{s}, 3 \mathrm{H}), 2.41(\mathrm{~s}, 3 \mathrm{H}), 1.07(\mathrm{~s}, 21 \mathrm{H}) ;{ }^{13} \mathrm{C} \mathrm{NMR}\left(126 \mathrm{MHz}, \mathrm{CDCl}_{3}\right) \delta 164.3,156.7,139.6$, 135.6, 134.5, 131.5, 130.9, 130.7, 122.7, 116.7, 114.0, 105.3, 98.5, 55.5, 21.4, 18.6, 11.3; HRMS calculated for $\mathrm{C}_{26} \mathrm{H}_{35} \mathrm{NO}_{2} \mathrm{Si}[\mathrm{M}+\mathrm{H}]^{+}:$422.2515, found 422.2498; Purification flash chromatography; Eluent:hexane/EtOAc $=95 / 05$.

\section{4,5-Dimethoxy- $N$-phenyl-2-((triisopropylsilyl)ethynyl)benzamide (5jo)}

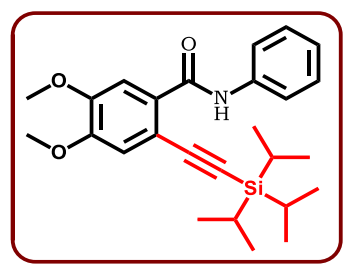

Fine white solid; (142 mg, 81\% yield); mp: $163-164^{\circ} \mathrm{C} ;{ }^{1} \mathrm{H}$ NMR $\left(500 \mathrm{MHz}, \mathrm{CDCl}_{3}\right) \delta 9.38$ (s, 1H), $7.70(\mathrm{~s}, 1 \mathrm{H}), 7.60(\mathrm{~d}, J=7.9 \mathrm{~Hz}, 2 \mathrm{H}), 7.35$ (t, $J=7.9 \mathrm{~Hz}, 2 \mathrm{H}), 7.15$ (t, $J=7.4 \mathrm{~Hz}$, $1 \mathrm{H}), 6.98(\mathrm{~s}, 1 \mathrm{H}), 3.96(\mathrm{~d}, J=6.7 \mathrm{~Hz}, 6 \mathrm{H}), 1.09(\mathrm{~d}, J=5.5 \mathrm{~Hz}, 21 \mathrm{H}) ;{ }^{13} \mathrm{C} \mathrm{NMR}(126 \mathrm{MHz}$, $\left.\mathrm{CDCl}_{3}\right) \delta 163.8,150.7,149.8,137.8,129.1,128.8,124.6,121.0,116.0,112.6,112.5,105.4$, 98.4, 56.1, 56.1, 18.6, 11.3; HRMS calculated for $\mathrm{C}_{26} \mathrm{H}_{35} \mathrm{NO}_{3} \mathrm{Si}[\mathrm{M}+\mathrm{H}]^{+}$: 438.2464, found 438.2447; Purification flash chromatography; Eluent: hexane/EtOAc $=93 / 07$. 


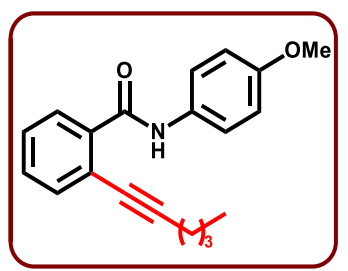

White solid; (104 mg, 85\% yield); mp: 88-89 ${ }^{\circ} \mathrm{C} ;{ }^{1} \mathrm{H}$ NMR $\left(500 \mathrm{MHz}, \mathrm{CDCl}_{3}\right) \delta 9.30(\mathrm{~s}, 1 \mathrm{H})$, $8.16-8.11(\mathrm{~m}, 1 \mathrm{H}), 7.60-7.55(\mathrm{~m}, 2 \mathrm{H}), 7.54-7.50(\mathrm{~m}, 1 \mathrm{H}), 7.44-7.40(\mathrm{~m}, 2 \mathrm{H}), 6.94-$ $6.90(\mathrm{~m}, 2 \mathrm{H}), 3.82(\mathrm{~s}, 3 \mathrm{H}), 2.52(\mathrm{t}, J=7.1 \mathrm{~Hz}, 2 \mathrm{H}), 1.64-1.59$ (m, 2H), 1.45 (dd, $J=15.0$, $7.4 \mathrm{~Hz}, 2 \mathrm{H}), 0.90(\mathrm{t}, J=7.3 \mathrm{~Hz}, 3 \mathrm{H}) ;{ }^{13} \mathrm{C} \mathrm{NMR}\left(126 \mathrm{MHz}, \mathrm{CDCl}_{3}\right) \delta 164.1,156.5,135.3$, 133.7, 131.2, 130.7, 130.2, 128.4, 121.8, 120.1, 114.2, 98.4, 79.3, 55.5, 30.6, 22.1, 19.4, 13.5; HRMS calculated for $\mathrm{C}_{20} \mathrm{H}_{21} \mathrm{NO}_{2}[\mathrm{M}+\mathrm{H}]^{+}:$308.1651, found 308.1634; Purification flash chromatography; Eluent: hexane/EtOAc $=93 / 07$.

\section{2-(Cyclohexylethynyl)- $N$-(4-methoxyphenyl)benzamide (5al)}

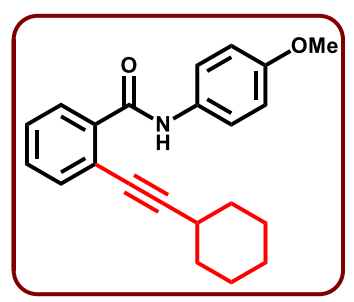

Pale brownish solid; (117 mg, 88\% yield); mp: 96-97 ${ }^{\circ} \mathrm{C} ;{ }^{1} \mathrm{H}$ NMR (500 MHz, $\left.\mathrm{CDCl}_{3}\right) \delta 9.27$ (s, 1H), $8.12(\mathrm{dd}, J=5.6,3.6 \mathrm{~Hz}, 1 \mathrm{H}), 7.59-7.55(\mathrm{~m}, 2 \mathrm{H}), 7.51$ (dd, $J=5.5,3.5 \mathrm{~Hz}, 1 \mathrm{H})$, $7.43-7.40(\mathrm{~m}, 2 \mathrm{H}), 6.93-6.90(\mathrm{~m}, 2 \mathrm{H}), 3.82(\mathrm{~s}, 3 \mathrm{H}), 2.72-2.66(\mathrm{~m}, 1 \mathrm{H}), 1.88$ (dd, $J=$ 10.4, $4.5 \mathrm{~Hz}, 2 \mathrm{H}), 1.75-1.70(\mathrm{~m}, 2 \mathrm{H}), 1.54(\mathrm{dd}, J=12.9,6.1 \mathrm{~Hz}, 3 \mathrm{H}), 1.33(\mathrm{dd}, J=11.6,9.3$ $\mathrm{Hz}, 3 \mathrm{H}) ;{ }^{13} \mathrm{C} \mathrm{NMR}\left(126 \mathrm{MHz}, \mathrm{CDCl}_{3}\right) \delta 164.19,156.49,135.22,133.79,131.07,130.62$, $130.19,128.28,121.97,120.17,114.17,102.32$, 79.24, 55.48, 55.46, 32.47, 29.90, 25.67, 24.78; HRMS calculated for $\mathrm{C}_{22} \mathrm{H}_{23} \mathrm{NO}_{2}[\mathrm{M}+\mathrm{H}]^{+}$: 334.1807, found 334.1806; Purification flash chromatography; Eluent: hexane/EtOAc $=93 / 07$.

\section{2-(Cyclopropylethynyl)- $N$-(4-methoxyphenyl)benzamide (5am)}

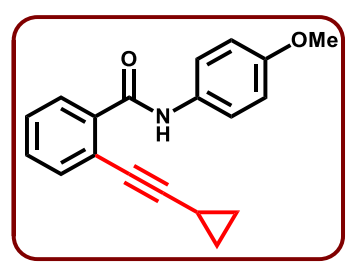


White solid; (92 mg, 79\% yield); mp: $129-130{ }^{\circ} \mathrm{C} ;{ }^{1} \mathrm{H}$ NMR $\left(500 \mathrm{MHz}, \mathrm{CDCl}_{3}\right) \delta 9.32$ (s, $1 \mathrm{H}), 8.16-8.09(\mathrm{~m}, 1 \mathrm{H}), 7.61(\mathrm{~d}, J=8.9 \mathrm{~Hz}, 2 \mathrm{H}), 7.51-7.46(\mathrm{~m}, 1 \mathrm{H}), 7.44-7.38(\mathrm{~m}, 2 \mathrm{H})$, $6.93(\mathrm{~d}, J=8.8 \mathrm{~Hz}, 2 \mathrm{H}), 3.82(\mathrm{~s}, 3 \mathrm{H}), 1.60-1.51(\mathrm{~m}, 1 \mathrm{H}), 0.99-0.91(\mathrm{~m}, 2 \mathrm{H}), 0.86-0.80$ $(\mathrm{m}, 2 \mathrm{H}) ;{ }^{13} \mathrm{C} \mathrm{NMR}\left(126 \mathrm{MHz}, \mathrm{CDCl}_{3}\right) \delta$ 164.0, 156.4, 135.4, 133.6, 131.2, 130.6, 130.2, $128.3,121.6,120.0,114.2,101.4,74.28,55.47,8.98$; HRMS calculated for $\mathrm{C}_{19} \mathrm{H}_{17} \mathrm{NO}_{2}$ $[\mathrm{M}+\mathrm{H}]^{+}: \quad 292.1338$, found 292.1337; Purification flash chromatography; Eluent: hexane $/$ EtOAc $=94 / 06$.

\section{2-(Cyclopentylethynyl)- $\mathrm{N}$-(4-methoxyphenyl)benzamide (5an)}

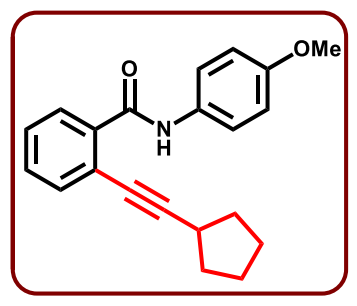

White solid; (111 mg, 87\% yield); mp: $113-114{ }^{\circ} \mathrm{C} ;{ }^{1} \mathrm{H}$ NMR (500 MHz, $\left.\mathrm{CDCl}_{3}\right) \delta 9.30$ (s, $1 \mathrm{H}), 8.14(\mathrm{dd}, J=5.8,3.5 \mathrm{~Hz}, 1 \mathrm{H}), 7.58(\mathrm{~d}, J=8.9 \mathrm{~Hz}, 2 \mathrm{H}), 7.51(\mathrm{dd}, J=5.6,3.4 \mathrm{~Hz}, 1 \mathrm{H})$, $7.42(\mathrm{dd}, J=5.8,3.4 \mathrm{~Hz}, 2 \mathrm{H}), 6.92(\mathrm{~d}, J=8.9 \mathrm{~Hz}, 2 \mathrm{H}), 3.82(\mathrm{~s}, 3 \mathrm{H}), 2.92$ (d, $J=7.6 \mathrm{~Hz}, 1 \mathrm{H})$, $2.02(\mathrm{~d}, J=8.0 \mathrm{~Hz}, 2 \mathrm{H}), 1.80-1.66(\mathrm{~m}, 4 \mathrm{H}), 1.60(\mathrm{dd}, J=9.6,5.1 \mathrm{~Hz}, 2 \mathrm{H}) ;{ }^{13} \mathrm{C} \mathrm{NMR}(126$ $\left.\mathrm{MHz}, \mathrm{CDCl}_{3}\right) \delta 164.2,156.5,135.2,133.7,131.2,130.7,130.3,128.3,121.9,120.2,114.2$, 102.6, 55.5, 33.9, 30.9, 25.1; HRMS calculated for $\mathrm{C}_{21} \mathrm{H}_{21} \mathrm{NO}_{2}[\mathrm{M}+\mathrm{H}]^{+}:$320.1651, found 320.1658; Purification flash chromatography; Eluent: hexane/EtOAc $=95 / 05$.

(Z)-3-Benzylideneisobenzofuran-1(3H)-one (6a)



White solid; (72 mg, 81\% yield); mp: $81-82{ }^{\circ} \mathrm{C} ;{ }^{1} \mathrm{H}$ NMR $\left(500 \mathrm{MHz}, \mathrm{CDCl}_{3}\right) \delta 7.94$ (dt, $J=$ 7.7, $0.8 \mathrm{~Hz}, 1 \mathrm{H}), 7.88-7.83(\mathrm{~m}, 2 \mathrm{H}), 7.78(\mathrm{dt}, J=7.8,0.8 \mathrm{~Hz}, 1 \mathrm{H}), 7.74(\mathrm{dd}, J=7.2,1.0 \mathrm{~Hz}$, 1H), 7.56 (dd, $J=10.9,4.0 \mathrm{~Hz}, 1 \mathrm{H}), 7.41$ (dd, $J=10.8,4.5 \mathrm{~Hz}, 2 \mathrm{H}), 7.33$ (d, $J=7.4 \mathrm{~Hz}, 1 \mathrm{H})$, $6.43(\mathrm{~s}, 1 \mathrm{H}) ;{ }^{13} \mathrm{C} \mathrm{NMR}\left(126 \mathrm{MHz}, \mathrm{CDCl}_{3}\right) \delta 167.1,144.5,140.6,134.5,133.0,130.1,129.8$, 128.8, 128.4, 125.6, 123.4, 119.8, 107.0; HRMS calculated for $\mathrm{C}_{15} \mathrm{H}_{10} \mathrm{O}_{2}[\mathrm{M}+\mathrm{H}]^{+}:$223.0759, found 223.0757; Purification flash chromatography; Eluent: hexane/EtOAc = 90/10. 


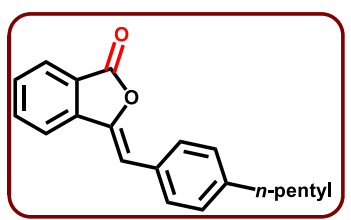

White solid; (99 mg, 85\% yield); mp: $110-11{ }^{\circ} \mathrm{C} ;{ }^{1} \mathrm{H}$ NMR $\left(500 \mathrm{MHz}, \mathrm{CDCl}_{3}\right) \delta 7.94(\mathrm{~d}, J=$ $7.7 \mathrm{~Hz}, 1 \mathrm{H}), 7.77$ (dd, $J=8.1,1.6 \mathrm{~Hz}, 3 \mathrm{H}), 7.75-7.69$ (m, 1H), 7.54 (t, $J=7.4 \mathrm{~Hz}, 1 \mathrm{H}), 7.23$ $(\mathrm{d}, J=8.2 \mathrm{~Hz}, 2 \mathrm{H}), 6.42(\mathrm{~s}, 1 \mathrm{H}), 2.66-2.60(\mathrm{~m}, 2 \mathrm{H}), 1.70-1.59(\mathrm{~m}, 2 \mathrm{H}), 1.33$ (dt, $J=9.7$, $3.7 \mathrm{~Hz}, 4 \mathrm{H}), 0.90$ (t, $J=7.0 \mathrm{~Hz}, 3 \mathrm{H}) ;{ }^{13} \mathrm{C} \mathrm{NMR}\left(126 \mathrm{MHz}, \mathrm{CDCl}_{3}\right) \delta 167.2,144.0,143.7$, $140.7,134.4,130.5,130.1,129.5,128.9,125.6$, 123.3, 119.7, 107.2, 35.8, 31.5, 31.0, 22.5, 14.0; HRMS calculated for $\mathrm{C}_{20} \mathrm{H}_{20} \mathrm{O}_{2}[\mathrm{M}+\mathrm{H}]^{+}:$293.1542, found 293.1540; Purification flash chromatography; Eluent: hexane/EtOAc $=98 / 02$.

(Z)-3-(4-Methoxybenzylidene)isobenzofuran-1(3H)-one (6c)

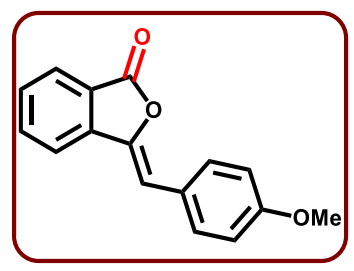

Yellow solid; (89 mg, 88\% yield); mp: $114-115^{\circ} \mathrm{C} ;{ }^{1} \mathrm{H}$ NMR $\left(500 \mathrm{MHz}, \mathrm{CDCl}_{3}\right) \delta 7.93(\mathrm{~d}, J$ $=7.7 \mathrm{~Hz}, 1 \mathrm{H}), 7.83-7.80(\mathrm{~m}, 2 \mathrm{H}), 7.71(\mathrm{ddd}, J=12.0,8.6,4.3 \mathrm{~Hz}, 2 \mathrm{H}), 7.54-7.49(\mathrm{~m}$, $1 \mathrm{H}), 6.97-6.93(\mathrm{~m}, 2 \mathrm{H}), 6.39(\mathrm{~s}, 1 \mathrm{H}), 3.86(\mathrm{~s}, 3 \mathrm{H}) ;{ }^{13} \mathrm{C} \mathrm{NMR}\left(126 \mathrm{MHz}, \mathrm{CDCl}_{3}\right) \delta 167.2$, $144.0,143.7,140.7,134.4,130.5,130.1,129.5$, 128.9, 125.6, 123.3, 119.7, 107.2 , 35.8, 31.4, 31.0, 22.5, 14.0; HRMS calculated for $\mathrm{C}_{16} \mathrm{H}_{12} \mathrm{O}_{3}[\mathrm{M}+\mathrm{H}]^{+}$: 253.0865, found 253.0864; Purification flash chromatography; Eluent: hexane/EtOAc $=98 / 02$.

(Z)-3-(3-Methylbenzylidene)isobenzofuran-1(3H)-one (6d)

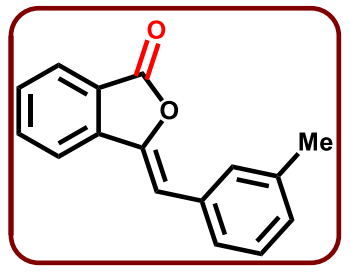

White solid; (74 mg, 79\% yield); mp: 100-101 ${ }^{\circ} \mathrm{C} ;{ }^{1} \mathrm{H}$ NMR (500 MHz, $\left.\mathrm{CDCl}_{3}\right) \delta 7.96-7.91$ (m, 1H), $7.78-7.75(\mathrm{~m}, 1 \mathrm{H}), 7.72(\mathrm{t}, J=7.5 \mathrm{~Hz}, 1 \mathrm{H}), 7.66(\mathrm{~d}, J=11.3 \mathrm{~Hz}, 2 \mathrm{H}), 7.54$ (t, $J=$ $7.4 \mathrm{~Hz}, 1 \mathrm{H}), 7.31$ (t, $J=7.6 \mathrm{~Hz}, 1 \mathrm{H}), 7.14(\mathrm{~d}, J=7.5 \mathrm{~Hz}, 1 \mathrm{H}), 6.40(\mathrm{~s}, 1 \mathrm{H}), 2.40(\mathrm{~s}, 3 \mathrm{H})$; ); ${ }^{13} \mathrm{C} \mathrm{NMR}\left(126 \mathrm{MHz}, \mathrm{CDCl}_{3}\right) \delta 167.1,144.4,140.6,138.4,134.4,132.9,130.6,129.7,129.3$, 128.6, 127.3, 125.5, 123.3, 119.7, 107.2, 21.5; HRMS calculated for $\mathrm{C}_{16} \mathrm{H}_{12} \mathrm{O}_{2}[\mathrm{M}+\mathrm{H}]^{+}$: 
237.0916, found 237.0914; Purification flash chromatography; Eluent: hexane/EtOAc = 98/02.

\section{3-Benzyl-2-(4-methoxyphenyl)isoindolin-1-one (7a)}

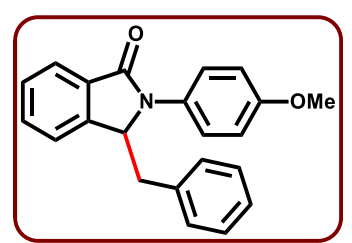

White solid; (128 mg, 97\% yield); mp: $138-139{ }^{\circ} \mathrm{C} ;{ }^{1} \mathrm{H}$ NMR $\left(500 \mathrm{MHz}, \mathrm{CDCl}_{3}\right) \delta 7.84$ (dd, $J=5.9,2.4 \mathrm{~Hz}, 1 \mathrm{H}), 7.52(\mathrm{~d}, J=8.9 \mathrm{~Hz}, 2 \mathrm{H}), 7.48-7.41(\mathrm{~m}, 2 \mathrm{H}), 7.21-7.13(\mathrm{~m}, 3 \mathrm{H}), 7.03$ $(\mathrm{dd}, J=6.9,4.7 \mathrm{~Hz}, 3 \mathrm{H}), 6.88(\mathrm{dd}, J=6.2,2.7 \mathrm{~Hz}, 2 \mathrm{H}), 5.36(\mathrm{dd}, J=8.2,3.7 \mathrm{~Hz}, 1 \mathrm{H}), 3.86$ $(\mathrm{s}, 3 \mathrm{H}), 3.36(\mathrm{dd}, J=13.8,3.6 \mathrm{~Hz}, 1 \mathrm{H}), 2.81(\mathrm{dd}, J=13.8,8.2 \mathrm{~Hz}, 1 \mathrm{H}) ;{ }^{13} \mathrm{C} \mathrm{NMR}(126 \mathrm{MHz}$, $\left.\mathrm{CDCl}_{3}\right) \delta 167.0,157.4,144.1,135.5,132.4,131.4,130.0,129.7,128.4,128.2,126.9,125.2$, 124.0, 123.0, 114.5, 61.9, 55.5, 38.2; HRMS calculated for $\mathrm{C}_{22} \mathrm{H}_{19} \mathrm{NO}_{2}[\mathrm{M}+\mathrm{H}]^{+}: 330.1494$, found 330.1495; Purification flash chromatography; Eluent: hexane/EtOAc = 90/10.

\section{2-(4-Methoxyphenyl)-3-(4-methylbenzyl)isoindolin-1-one (7b)}

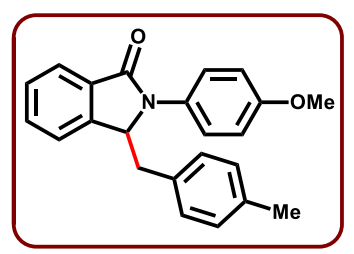

White solid; (130 mg, 95\% yield); mp: $133-134{ }^{\circ} \mathrm{C} ;{ }^{1} \mathrm{H}$ NMR $\left(500 \mathrm{MHz}, \mathrm{CDCl}_{3}\right) \delta 7.83(\mathrm{~d}, J$ $=6.7 \mathrm{~Hz}, 1 \mathrm{H}), 7.51(\mathrm{~d}, J=8.1 \mathrm{~Hz}, 2 \mathrm{H}), 7.45(\mathrm{p}, J=7.2 \mathrm{~Hz}, 2 \mathrm{H}), 7.04(\mathrm{dd}, J=17.0,7.5 \mathrm{~Hz}$, $3 \mathrm{H}), 6.97(\mathrm{~d}, J=7.3 \mathrm{~Hz}, 2 \mathrm{H}), 6.75(\mathrm{~d}, J=7.3 \mathrm{~Hz}, 2 \mathrm{H}), 5.34$ (s, 1H), $3.86(\mathrm{~s}, 3 \mathrm{H}), 3.32$ (d, $J=$ 13.7 Hz, 1H), 2.77 (dd, $J=13.6,8.4 \mathrm{~Hz}, 1 \mathrm{H}), 2.27(\mathrm{~s}, 3 \mathrm{H}) ;{ }^{13} \mathrm{C} \mathrm{NMR}\left(126 \mathrm{MHz}, \mathrm{CDCl}_{3}\right) \delta$ $167.0,157.3,144.2,136.4,132.4,132.2,131.3,130.0,129.5,128.9,128.3,125.2,123.9$, 123.0, 114.4, 61.9, 55.5, 37.7, 21.0; HRMS calculated for $\mathrm{C}_{23} \mathrm{H}_{21} \mathrm{NO}_{2}[\mathrm{M}+\mathrm{H}]^{+}: 344.1651$, found: 344.1634; Purification flash chromatography; Eluent: hexane/EtOAc =90/10.

$\mathrm{N}$-(4-Methoxyphenyl)-2-phenethylbenzamide (8a)

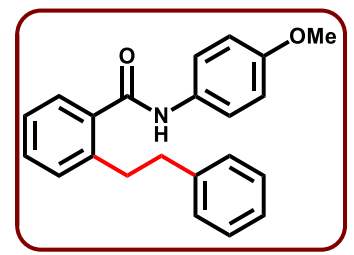

White solid (130 mg, 98\% yield); mp: $147-148{ }^{\circ} \mathrm{C} ;{ }^{1} \mathrm{H}$ NMR $\left(500 \mathrm{MHz}, \mathrm{CDCl}_{3}\right) \delta 7.44(\mathrm{~d}, J$ $=9.0 \mathrm{~Hz}, 2 \mathrm{H}), 7.41(\mathrm{~s}, 1 \mathrm{H}), 7.38(\mathrm{t}, J=7.5 \mathrm{~Hz}, 1 \mathrm{H}), 7.27(\mathrm{dd}, J=12.6,7.6 \mathrm{~Hz}, 2 \mathrm{H}), 7.20(\mathrm{t}, J$ $=7.2 \mathrm{~Hz}, 2 \mathrm{H}), 7.17-7.12(\mathrm{~m}, 1 \mathrm{H}), 7.09(\mathrm{~d}, J=7.2 \mathrm{~Hz}, 3 \mathrm{H}), 6.89(\mathrm{~d}, J=8.3 \mathrm{~Hz}, 2 \mathrm{H}), 3.81(\mathrm{~s}$, 
$3 \mathrm{H}), 3.14(\mathrm{t}, J=7.7 \mathrm{~Hz}, 2 \mathrm{H}), 2.96(\mathrm{t}, J=7.7 \mathrm{~Hz}, 2 \mathrm{H}) ;{ }^{13} \mathrm{C} \mathrm{NMR}\left(126 \mathrm{MHz}, \mathrm{CDCl}_{3}\right) \delta 167.9$, $156.5,141.5,139.9,136.8,130.9,130.5,130.1,128.7,128.3,126.6,126.1,125.9,121.8$, 114.1, 55.5, 38.0, 35.1; HRMS calculated for $\mathrm{C}_{22} \mathrm{H}_{21} \mathrm{NO}_{2}[\mathrm{M}+\mathrm{H}]^{+}:$332.1651, found: 332.1646; Purification flash chromatography; Eluent: hexane/EtOAc $=90 / 10$.

$N$-(4-Methoxyphenyl)-2-(4-methylphenethyl)benzamide (8b)

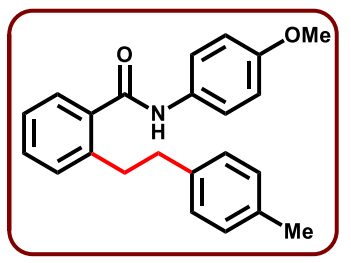

White solid (134 mg, 97\% yield); mp: $159-160^{\circ} \mathrm{C} ;{ }^{1} \mathrm{H}$ NMR (500 MHz, $\left.\mathrm{CDCl}_{3}\right) \delta 7.40$ (dd, $J$ $=17.4,7.6 \mathrm{~Hz}, 4 \mathrm{H}), 7.30(\mathrm{~d}, J=7.4 \mathrm{~Hz}, 1 \mathrm{H}), 7.25(\mathrm{~d}, J=7.3 \mathrm{~Hz}, 1 \mathrm{H}), 7.04-6.94(\mathrm{~m}, 5 \mathrm{H})$, $6.89(\mathrm{~d}, J=7.6 \mathrm{~Hz}, 2 \mathrm{H}), 3.82(\mathrm{~s}, 3 \mathrm{H}), 3.12(\mathrm{t}, J=7.1 \mathrm{~Hz}, 2 \mathrm{H}), 2.93(\mathrm{t}, J=7.2 \mathrm{~Hz}, 2 \mathrm{H}), 2.27$ $(\mathrm{s}, 3 \mathrm{H}) ;{ }^{13} \mathrm{C} \mathrm{NMR}\left(126 \mathrm{MHz}, \mathrm{CDCl}_{3}\right) \delta 167.9,156.5,139.9,138.4,136.9,135.3,131.0$, $130.5,130.1,129.0,128.6,126.6,126.1,121.8,114.1,55.5,37.6,35.2,21.0$; HRMS calculated for $\mathrm{C}_{23} \mathrm{H}_{23} \mathrm{NO}_{2}[\mathrm{M}+\mathrm{H}]^{+}:$346.1807, found: 346.1800; Purification flash chromatography; Eluent: hexane/EtOAc $=90 / 10$. 


\section{Single crystal X-Ray Diffraction data}

X-ray data of (Z)-2-(4-Methoxyphenyl)-3-(4-methylbenzylidene)isoindolin-1-one (3ab)

The crystal structure of compound 3ab has been deposited at the Cambridge Crystallographic Data Centre and allocated the deposition number CCDC: 1963275 (Figure S1).

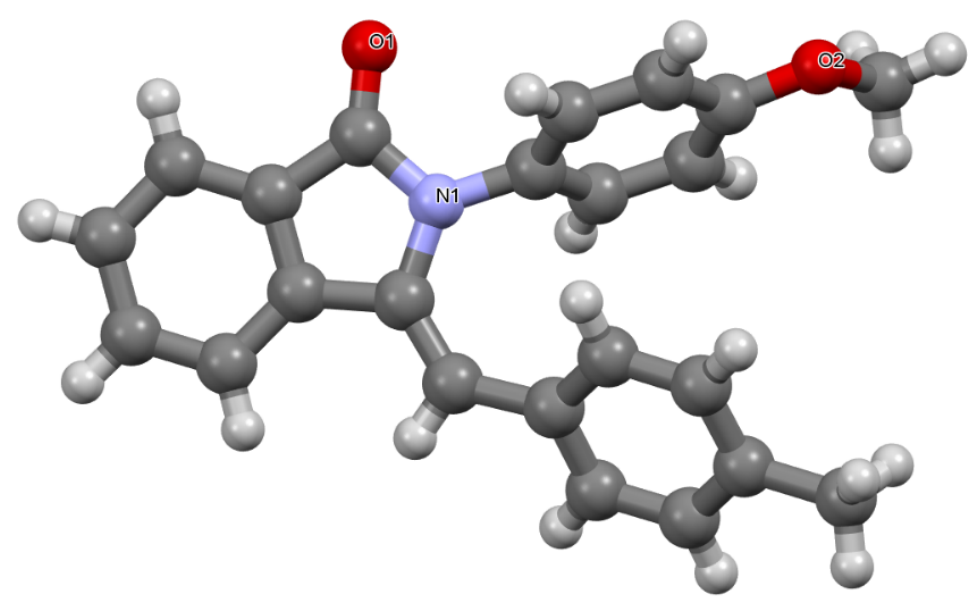

Table 2. Crystal data and structure refinement for $3 \mathrm{ab}$.

Identification code

Chemical formula

Formula weight

Temperature

Wavelength

Crystal size

Crystal habit

Crystal system

Space group

Unit cell dimensions

Volume

Z

Density (calculated)

Absorption coefficient

$\mathrm{F}(000)$

Theta range for data collection

Index ranges

Reflections collected

Independent reflections
SRM_Hari_474_S3

$\mathrm{C}_{23} \mathrm{H}_{19} \mathrm{NO}_{2}$

$341.39 \mathrm{~g} / \mathrm{mol}$

296(2) K

$0.71073 \AA$

$0.190 \times 0.220 \times 0.250 \mathrm{~mm}$

clear light colourless Block

monoclinic

P 1 21/n 1

$\mathrm{a}=6.4095(3) \AA \quad \alpha=90^{\circ}$

$\mathrm{b}=16.5475(6) \AA \quad \beta=92.7630(18)^{\circ}$

$\mathrm{c}=17.1513(7) \AA \quad \gamma=90^{\circ}$

1816.97(13) $\AA^{3}$

4

$1.248 \mathrm{~g} / \mathrm{cm}^{3}$

$0.079 \mathrm{~mm}^{-1}$

720

2.38 to $25.00^{\circ}$

$-7<=\mathrm{h}<=7,-15<=\mathrm{k}<=19,-20<=1<=20$

9352

$3205[\mathrm{R}(\mathrm{int})=0.0234]$ 
Coverage of independent reflections

Absorption correction

Max. and min. transmission

Refinement method

Refinement program

Function minimized

Data / restraints / parameters

Goodness-of-fit on $\mathrm{F}^{2}$

$\Delta / \sigma_{\max }$

Final $\mathrm{R}$ indices

Weighting scheme

Extinction coefficient

Largest diff. peak and hole

R.M.S. deviation from mean
$100.0 \%$

multi-scan

0.9850 and 0.9800

Full-matrix least-squares on $\mathrm{F}^{2}$

SHELXL-2014/7 (Sheldrick, 2014)

$\Sigma \mathrm{w}\left(\mathrm{F}_{\mathrm{o}}{ }^{2}-\mathrm{F}_{\mathrm{c}}{ }^{2}\right)^{2}$

3205 / 0 / 238

1.037

0.002

2306 data; $\mathrm{I}>2 \sigma(\mathrm{I})$

$\mathrm{R} 1=0.0414, \mathrm{wR} 2=$ 0.1000

all data

$\mathrm{R} 1=0.0645, \mathrm{wR} 2=$ 0.1149

$\mathrm{W}=1 /\left[\sigma^{2}\left(\mathrm{~F}_{\mathrm{o}}{ }^{2}\right)+(0.0522 \mathrm{P})^{2}+0.2966 \mathrm{P}\right]$

where $\mathrm{P}=\left(\mathrm{F}_{\mathrm{o}}^{2}+2 \mathrm{~F}_{\mathrm{c}}^{2}\right) / 3$

$0.0045(11)$

0.146 and $-0.126 \mathrm{e}^{-3}$

0.031 e $\AA^{-3}$

Table 3. Atomic coordinates and equivalent isotropic atomic displacement parameters $\left(\AA^{2}\right)$ for 3ab.

$\mathrm{U}(\mathrm{eq})$ is defined as one third of the trace of the orthogonalized $\mathrm{U}_{\mathrm{ij}}$ tensor.

$\begin{array}{lllll} & \mathbf{x} / \mathbf{a} & \mathbf{y} / \mathbf{b} & \mathbf{z} / \mathbf{c} & \mathbf{U}(\mathbf{e q}) \\ \text { C1 } & 0.9642(3) & 0.88718(9) & 0.87721(10) & 0.0444(4) \\ \text { C2 } & 0.0946(3) & 0.94461(10) & 0.83610(10) & 0.0489(5) \\ \text { C3 } & 0.0955(4) & 0.02874(11) & 0.83550(12) & 0.0668(6) \\ \text { C4 } & 0.2475(4) & 0.06727(13) & 0.79617(13) & 0.0771(7) \\ \text { C5 } & 0.3984(4) & 0.02513(14) & 0.75814(13) & 0.0760(7) \\ \text { C6 } & 0.3981(3) & 0.94166(13) & 0.75759(12) & 0.0634(5) \\ \text { C7 } & 0.2439(3) & 0.90272(10) & 0.79699(10) & 0.0476(4) \\ \text { C8 } & 0.2080(3) & 0.81591(10) & 0.80804(10) & 0.0447(4) \\ \text { C9 } & 0.9540(3) & 0.73307(9) & 0.87531(10) & 0.0404(4) \\ \text { C10 } & 0.0719(3) & 0.67708(10) & 0.91853(10) & 0.0474(5) \\ \text { C11 } & 0.9877(3) & 0.60334(10) & 0.93547(11) & 0.0518(5) \\ \text { C12 } & 0.7860(3) & 0.58414(10) & 0.90886(10) & 0.0471(4) \\ \text { C13 } & 0.6686(3) & 0.63989(10) & 0.86594(10) & 0.0467(4) \\ \text { C14 } & 0.7546(3) & 0.71431(10) & 0.84959(10) & 0.0429(4) \\ \text { C15 } & 0.5210(3) & 0.48306(13) & 0.89583(15) & 0.0792(7) \\ \text { C16 } & 0.8111(3) & 0.90741(10) & 0.92396(11) & 0.0521(5)\end{array}$




\begin{tabular}{lllll} 
& $\mathbf{x} / \mathbf{a}$ & $\mathbf{y} / \mathbf{b}$ & $\mathbf{z} / \mathbf{c}$ & $\mathbf{U}(\mathbf{e q})$ \\
$\mathrm{C} 17$ & $0.6812(3)$ & $0.86203(10)$ & $0.97714(10)$ & $0.0463(4)$ \\
$\mathrm{C} 18$ & $0.7539(3)$ & $0.79757(11)$ & $0.02296(11)$ & $0.0527(5)$ \\
$\mathrm{C} 19$ & $0.6269(3)$ & $0.75974(11)$ & $0.07441(11)$ & $0.0560(5)$ \\
$\mathrm{C} 20$ & $0.4232(3)$ & $0.78428(12)$ & $0.08265(11)$ & $0.0580(5)$ \\
$\mathrm{C} 21$ & $0.3519(3)$ & $0.84918(12)$ & $0.03778(12)$ & $0.0588(5)$ \\
$\mathrm{C} 22$ & $0.4778(3)$ & $0.88781(11)$ & $0.98700(11)$ & $0.0521(5)$ \\
$\mathrm{C} 23$ & $0.2850(4)$ & $0.74231(17)$ & $0.13915(14)$ & $0.0906(8)$ \\
$\mathrm{N} 1$ & $0.0430(2)$ & $0.80999(8)$ & $0.85785(8)$ & $0.0434(4)$ \\
$\mathrm{O} 1$ & $0.29671(19)$ & $0.75870(7)$ & $0.77984(8)$ & $0.0591(4)$ \\
$\mathrm{O} 2$ & $0.7187(2)$ & $0.50821(7)$ & $0.92724(9)$ & $0.0682(4)$ \\
\hline
\end{tabular}

Table 4. Bond lengths (Å) for 3ab.

\begin{tabular}{|c|c|c|c|}
\hline $\mathrm{C} 1-\mathrm{C} 16$ & $1.339(2)$ & $\mathrm{C} 1-\mathrm{N} 1$ & $1.419(2)$ \\
\hline $\mathrm{C} 1-\mathrm{C} 2$ & $1.468(2)$ & $\mathrm{C} 2-\mathrm{C} 7$ & $1.382(2)$ \\
\hline $\mathrm{C} 2-\mathrm{C} 3$ & $1.392(2)$ & $\mathrm{C} 3-\mathrm{C} 4$ & $1.369(3)$ \\
\hline C3-H3 & 0.93 & $\mathrm{C} 4-\mathrm{C} 5$ & $1.381(3)$ \\
\hline $\mathrm{C} 4-\mathrm{H} 4$ & 0.93 & $\mathrm{C} 5-\mathrm{C} 6$ & $1.381(3)$ \\
\hline $\mathrm{C} 5-\mathrm{H} 5$ & 0.93 & C6-C7 & $1.383(3)$ \\
\hline C6-H6 & 0.93 & $\mathrm{C} 7-\mathrm{C} 8$ & $1.468(2)$ \\
\hline $\mathrm{C} 8-\mathrm{O} 1$ & $1.217(2)$ & $\mathrm{C} 8-\mathrm{N} 1$ & $1.394(2)$ \\
\hline C9-C14 & $1.368(2)$ & C9-C10 & $1.387(2)$ \\
\hline C9-N1 & $1.432(2)$ & C10-C11 & $1.371(2)$ \\
\hline $\mathrm{C} 10-\mathrm{H} 10$ & 0.93 & $\mathrm{C} 11-\mathrm{C} 12$ & $1.387(2)$ \\
\hline C11-H11 & 0.93 & $\mathrm{C} 12-\mathrm{O} 2$ & $1.370(2)$ \\
\hline $\mathrm{C} 12-\mathrm{C} 13$ & $1.380(2)$ & C13-C14 & $1.383(2)$ \\
\hline C13-H13 & 0.93 & C14-H14 & 0.93 \\
\hline $\mathrm{C} 15-\mathrm{O} 2$ & $1.415(2)$ & C15-H15A & 0.96 \\
\hline C15-H15B & 0.96 & $\mathrm{C} 15-\mathrm{H} 15 \mathrm{C}$ & 0.96 \\
\hline $\mathrm{C} 16-\mathrm{C} 17$ & $1.470(2)$ & C16-H16 & 0.93 \\
\hline $\mathrm{C} 17-\mathrm{C} 22$ & $1.390(2)$ & $\mathrm{C} 17-\mathrm{C} 18$ & $1.392(2)$ \\
\hline C18-C19 & $1.379(3)$ & C18-H18 & 0.93 \\
\hline C19-C20 & $1.381(3)$ & C19-H19 & 0.93 \\
\hline C20-C21 & $1.386(3)$ & $\mathrm{C} 20-\mathrm{C} 23$ & $1.513(3)$ \\
\hline $\mathrm{C} 21-\mathrm{C} 22$ & $1.373(3)$ & $\mathrm{C} 21-\mathrm{H} 21$ & 0.93 \\
\hline C22-H22 & 0.93 & $\mathrm{C} 23-\mathrm{H} 23 \mathrm{~A}$ & 0.96 \\
\hline $\mathrm{C} 23-\mathrm{H} 23 \mathrm{~B}$ & 0.96 & $\mathrm{C} 23-\mathrm{H} 23 \mathrm{C}$ & 0.96 \\
\hline
\end{tabular}


Table 5. Bond angles $\left({ }^{\circ}\right)$ for $3 a b$.

\begin{tabular}{|c|c|c|c|}
\hline C16-C1-N1 & $130.13(16)$ & $\mathrm{C} 16-\mathrm{C} 1-\mathrm{C} 2$ & $125.19(16)$ \\
\hline $\mathrm{N} 1-\mathrm{C} 1-\mathrm{C} 2$ & $104.64(14)$ & $\mathrm{C} 7-\mathrm{C} 2-\mathrm{C} 3$ & $119.67(18)$ \\
\hline $\mathrm{C} 7-\mathrm{C} 2-\mathrm{C} 1$ & $109.41(15)$ & $\mathrm{C} 3-\mathrm{C} 2-\mathrm{C} 1$ & $130.80(18)$ \\
\hline $\mathrm{C} 4-\mathrm{C} 3-\mathrm{C} 2$ & $118.2(2)$ & $\mathrm{C} 4-\mathrm{C} 3-\mathrm{H} 3$ & 120.9 \\
\hline $\mathrm{C} 2-\mathrm{C} 3-\mathrm{H} 3$ & 120.9 & $\mathrm{C} 3-\mathrm{C} 4-\mathrm{C} 5$ & $121.9(2)$ \\
\hline $\mathrm{C} 3-\mathrm{C} 4-\mathrm{H} 4$ & 119.0 & $\mathrm{C} 5-\mathrm{C} 4-\mathrm{H} 4$ & 119.0 \\
\hline $\mathrm{C} 4-\mathrm{C} 5-\mathrm{C} 6$ & $120.5(2)$ & $\mathrm{C} 4-\mathrm{C} 5-\mathrm{H} 5$ & 119.8 \\
\hline C6-C5-H5 & 119.8 & $\mathrm{C} 5-\mathrm{C} 6-\mathrm{C} 7$ & $117.6(2)$ \\
\hline C5-C6-H6 & 121.2 & C7-C6-H6 & 121.2 \\
\hline C2-C7-C6 & $122.11(18)$ & $\mathrm{C} 2-\mathrm{C} 7-\mathrm{C} 8$ & $108.14(15)$ \\
\hline C6-C7-C8 & $129.73(18)$ & $\mathrm{O} 1-\mathrm{C} 8-\mathrm{N} 1$ & $124.86(15)$ \\
\hline $\mathrm{O} 1-\mathrm{C} 8-\mathrm{C} 7$ & $129.08(17)$ & $\mathrm{N} 1-\mathrm{C} 8-\mathrm{C} 7$ & $106.04(14)$ \\
\hline C14-C9-C10 & $119.82(15)$ & C14-C9-N1 & $120.64(14)$ \\
\hline C10-C9-N1 & $119.53(15)$ & C11-C10-C9 & $119.84(16)$ \\
\hline C11-C10-H10 & 120.1 & C9-C10-H10 & 120.1 \\
\hline C10-C11-C12 & $120.30(15)$ & C10-C11-H11 & 119.8 \\
\hline C12-C11-H11 & 119.8 & $\mathrm{O} 2-\mathrm{C} 12-\mathrm{C} 13$ & $124.48(16)$ \\
\hline $\mathrm{O} 2-\mathrm{C} 12-\mathrm{C} 11$ & $115.67(14)$ & C13-C12-C11 & $119.84(16)$ \\
\hline C12-C13-C14 & 119.41(16) & C12-C13-H13 & 120.3 \\
\hline C14-C13-H13 & 120.3 & C9-C14-C13 & $120.78(15)$ \\
\hline C9-C14-H14 & 119.6 & C13-C14-H14 & 119.6 \\
\hline $\mathrm{O} 2-\mathrm{C} 15-\mathrm{H} 15 \mathrm{~A}$ & 109.5 & $\mathrm{O} 2-\mathrm{C} 15-\mathrm{H} 15 \mathrm{~B}$ & 109.5 \\
\hline H15A-C15-H15B & 109.5 & $\mathrm{O} 2-\mathrm{C} 15-\mathrm{H} 15 \mathrm{C}$ & 109.5 \\
\hline H15A-C15-H15C & 109.5 & H15B-C15-H15C & 109.5 \\
\hline C1-C16-C17 & $134.17(16)$ & C1-C16-H16 & 112.9 \\
\hline C17-C16-H16 & 112.9 & C22-C17-C18 & $117.20(17)$ \\
\hline C22-C17-C16 & $118.76(16)$ & C18-C17-C16 & $123.88(16)$ \\
\hline C19-C18-C17 & $121.15(17)$ & C19-C18-H18 & 119.4 \\
\hline C17-C18-H18 & 119.4 & C18-C19-C20 & $121.46(18)$ \\
\hline C18-C19-H19 & 119.3 & C20-C19-H19 & 119.3 \\
\hline C19-C20-C21 & $117.34(18)$ & C19-C20-C23 & $121.1(2)$ \\
\hline $\mathrm{C} 21-\mathrm{C} 20-\mathrm{C} 23$ & $121.57(19)$ & C22-C21-C20 & $121.65(18)$ \\
\hline C22-C21-H21 & 119.2 & C20-C21-H21 & 119.2 \\
\hline $\mathrm{C} 21-\mathrm{C} 22-\mathrm{C} 17$ & $121.17(18)$ & $\mathrm{C} 21-\mathrm{C} 22-\mathrm{H} 22$ & 119.4 \\
\hline C17-C22-H22 & 119.4 & C20-C23-H23A & 109.5 \\
\hline $\mathrm{C} 20-\mathrm{C} 23-\mathrm{H} 23 \mathrm{~B}$ & 109.5 & $\mathrm{H} 23 \mathrm{~A}-\mathrm{C} 23-\mathrm{H} 23 \mathrm{~B}$ & 109.5 \\
\hline $\mathrm{C} 20-\mathrm{C} 23-\mathrm{H} 23 \mathrm{C}$ & 109.5 & $\mathrm{H} 23 \mathrm{~A}-\mathrm{C} 23-\mathrm{H} 23 \mathrm{C}$ & 109.5 \\
\hline H23B-C23-H23C & 109.5 & C8-N1-C1 & $111.67(13)$ \\
\hline
\end{tabular}


Table 6. Torsion angles $\left({ }^{\circ}\right)$ for $3 a b$.

\begin{tabular}{|c|c|c|c|}
\hline $\mathrm{C} 16-\mathrm{C} 1-\mathrm{C} 2-\mathrm{C} 7$ & $176.53(17)$ & $\mathrm{N} 1-\mathrm{C} 1-\mathrm{C} 2-\mathrm{C} 7$ & $-1.55(19)$ \\
\hline $\mathrm{C} 16-\mathrm{C} 1-\mathrm{C} 2-\mathrm{C} 3$ & $0.8(3)$ & $\mathrm{N} 1-\mathrm{C} 1-\mathrm{C} 2-\mathrm{C} 3$ & $-177.29(19)$ \\
\hline $\mathrm{C} 7-\mathrm{C} 2-\mathrm{C} 3-\mathrm{C} 4$ & $-0.5(3)$ & $\mathrm{C} 1-\mathrm{C} 2-\mathrm{C} 3-\mathrm{C} 4$ & $174.84(19)$ \\
\hline $\mathrm{C} 2-\mathrm{C} 3-\mathrm{C} 4-\mathrm{C} 5$ & $-0.4(3)$ & $\mathrm{C} 3-\mathrm{C} 4-\mathrm{C} 5-\mathrm{C} 6$ & $1.1(3)$ \\
\hline C4-C5-C6-C7 & $-0.8(3)$ & $\mathrm{C} 3-\mathrm{C} 2-\mathrm{C} 7-\mathrm{C} 6$ & $0.9(3)$ \\
\hline $\mathrm{C} 1-\mathrm{C} 2-\mathrm{C} 7-\mathrm{C} 6$ & $-175.40(16)$ & $\mathrm{C} 3-\mathrm{C} 2-\mathrm{C} 7-\mathrm{C} 8$ & $179.32(16)$ \\
\hline $\mathrm{C} 1-\mathrm{C} 2-\mathrm{C} 7-\mathrm{C} 8$ & $3.03(19)$ & $\mathrm{C} 5-\mathrm{C} 6-\mathrm{C} 7-\mathrm{C} 2$ & $-0.2(3)$ \\
\hline C5-C6-C7-C8 & $-178.29(18)$ & $\mathrm{C} 2-\mathrm{C} 7-\mathrm{C} 8-\mathrm{O} 1$ & $175.44(18)$ \\
\hline C6-C7-C8-O1 & $-6.3(3)$ & $\mathrm{C} 2-\mathrm{C} 7-\mathrm{C} 8-\mathrm{N} 1$ & $-3.34(18)$ \\
\hline C6-C7-C8-N1 & $174.93(17)$ & C14-C9-C10-C11 & $-0.2(3)$ \\
\hline N1-C9-C10-C11 & $179.98(16)$ & C9-C10-C11-C12 & $0.7(3)$ \\
\hline $\mathrm{C} 10-\mathrm{C} 11-\mathrm{C} 12-\mathrm{O} 2$ & $178.35(16)$ & $\mathrm{C} 10-\mathrm{C} 11-\mathrm{C} 12-\mathrm{C} 13$ & $-0.8(3)$ \\
\hline $\mathrm{O} 2-\mathrm{C} 12-\mathrm{C} 13-\mathrm{C} 14$ & $-178.70(16)$ & C11-C12-C13-C14 & $0.4(3)$ \\
\hline C10-C9-C14-C13 & $-0.2(3)$ & N1-C9-C14-C13 & $179.59(15)$ \\
\hline C12-C13-C14-C9 & $0.1(3)$ & N1-C1-C16-C17 & $6.3(3)$ \\
\hline $\mathrm{C} 2-\mathrm{C} 1-\mathrm{C} 16-\mathrm{C} 17$ & $-171.22(18)$ & $\mathrm{C} 1-\mathrm{C} 16-\mathrm{C} 17-\mathrm{C} 22$ & $-148.0(2)$ \\
\hline C1-C16-C17-C18 & $36.8(3)$ & C22-C17-C18-C19 & $1.6(3)$ \\
\hline C16-C17-C18-C19 & $177.00(17)$ & C17-C18-C19-C20 & $-0.1(3)$ \\
\hline C18-C19-C20-C21 & $-0.7(3)$ & C18-C19-C20-C23 & 179.90(19) \\
\hline C19-C20-C21-C22 & $0.0(3)$ & $\mathrm{C} 23-\mathrm{C} 20-\mathrm{C} 21-\mathrm{C} 22$ & 179.39(19) \\
\hline C20-C21-C22-C17 & $1.6(3)$ & C18-C17-C22-C21 & $-2.3(3)$ \\
\hline C16-C17-C22-C21 & $-177.95(16)$ & O1-C8-N1-C1 & $-176.43(16)$ \\
\hline C7-C8-N1-C1 & $2.42(18)$ & O1-C8-N1-C9 & $-4.7(2)$ \\
\hline C7-C8-N1-C9 & $174.18(13)$ & C16-C1-N1-C8 & $-178.58(18)$ \\
\hline $\mathrm{C} 2-\mathrm{C} 1-\mathrm{N} 1-\mathrm{C} 8$ & $-0.64(18)$ & C16-C1-N1-C9 & $10.3(3)$ \\
\hline C2-C1-N1-C9 & $-171.78(14)$ & C14-C9-N1-C8 & $-112.43(18)$ \\
\hline C10-C9-N1-C8 & $67.4(2)$ & C14-C9-N1-C1 & $58.0(2)$ \\
\hline C10-C9-N1-C1 & $-122.23(18)$ & $\mathrm{C} 13-\mathrm{C} 12-\mathrm{O} 2-\mathrm{C} 15$ & $4.4(3)$ \\
\hline $\mathrm{C} 11-\mathrm{C} 12-\mathrm{O} 2-\mathrm{C} 15$ & $-174.67(18)$ & & \\
\hline
\end{tabular}

\section{Table 7. Anisotropic atomic displacement parameters $\left(\AA^{2}\right)$ for $3 a b$.}

The anisotropic atomic displacement factor exponent takes the form: $-2 \pi^{2}\left[h^{2} a^{* 2} U_{11}+\ldots\right.$ $\left.+2 \mathrm{~h} \mathrm{k} \mathrm{a}^{*} \mathrm{~b}^{*} \mathrm{U}_{12}\right]$

\begin{tabular}{lcccccc} 
& $\mathbf{U}_{\mathbf{1 1}}$ & $\mathbf{U}_{\mathbf{2 2}}$ & \multicolumn{1}{c}{$\mathbf{U}_{\mathbf{3 3}}$} & $\mathbf{U}_{\mathbf{2 3}}$ & $\mathbf{U}_{\mathbf{1 3}}$ & $\mathbf{U}_{\mathbf{1 2}}$ \\
$\mathrm{C} 1$ & $0.0505(10)$ & $0.0398(9)$ & $0.0431(10)$ & $0.0024(7)$ & $0.0041(8)$ & $0.0064(8)$ \\
$\mathrm{C} 2$ & $0.0614(12)$ & $0.0427(9)$ & $0.0429(10)$ & $0.0043(8)$ & $0.0044(9)$ & $0.0008(8)$ \\
C3 & $0.0959(16)$ & $0.0458(11)$ & $0.0597(13)$ & $0.0028(9)$ & $0.0157(12)$ & $-0.0019(10)$
\end{tabular}


$\begin{array}{llllll}\mathbf{U}_{11} & \mathbf{U}_{22} & \mathbf{U}_{33} & \mathbf{U}_{23} & \mathbf{U}_{13} & \mathbf{U}_{12}\end{array}$

$\begin{array}{lllllll}\text { C4 } & 0.113(2) & 0.0526(12) & 0.0661(15) & 0.0081(11) & 0.0093(14) & -0.0174(13) \\ \text { C5 } & 0.0871(17) & 0.0807(16) & 0.0610(14) & 0.0154(12) & 0.0116(12) & -0.0313(13) \\ \text { C6 } & 0.0630(13) & 0.0739(14) & 0.0540(12) & 0.0062(10) & 0.0094(10) & -0.0107(11) \\ \text { C7 } & 0.0491(11) & 0.0536(11) & 0.0402(10) & 0.0051(8) & 0.0020(8) & -0.0030(9) \\ \text { C8 } & 0.0408(10) & 0.0494(10) & 0.0437(10) & 0.0004(8) & 0.0010(8) & 0.0050(8) \\ \text { C9 } & 0.0422(9) & 0.0380(8) & 0.0411(10) & 0.0010(7) & 0.0035(7) & 0.0046(7) \\ \text { C10 } & 0.0419(10) & 0.0507(10) & 0.0488(11) & 0.0037(8) & -0.0049(8) & 0.0049(8) \\ \text { C11 } & 0.0505(11) & 0.0489(10) & 0.0554(12) & 0.0147(8) & -0.0032(9) & 0.0089(9) \\ \text { C12 } & 0.0504(11) & 0.0439(10) & 0.0473(11) & 0.0082(8) & 0.0053(8) & 0.0011(8) \\ \text { C13 } & 0.0411(10) & 0.0511(10) & 0.0479(11) & 0.0024(8) & 0.0001(8) & -0.0009(8) \\ \text { C14 } & 0.0433(10) & 0.0448(9) & 0.0405(10) & 0.0051(7) & 0.0006(8) & 0.0087(8) \\ \text { C15 } & 0.0734(15) & 0.0651(13) & 0.0987(18) & 0.0105(12) & -0.0006(13) & -0.0223(11) \\ \text { C16 } & 0.0596(11) & 0.0421(9) & 0.0552(11) & 0.0003(8) & 0.0093(9) & 0.0084(9) \\ \text { C17 } & 0.0509(11) & 0.0455(9) & 0.0429(10) & -0.0071(8) & 0.0075(8) & 0.0021(8) \\ \text { C18 } & 0.0494(11) & 0.0601(11) & 0.0489(11) & -0.0007(9) & 0.0072(9) & 0.0067(9) \\ \text { C19 } & 0.0651(13) & 0.0614(11) & 0.0420(11) & 0.0040(9) & 0.0086(9) & 0.0022(10) \\ \text { C20 } & 0.0580(12) & 0.0746(13) & 0.0421(11) & -0.0074(10) & 0.0108(9) & -0.0100(10) \\ \text { C21 } & 0.0468(11) & 0.0738(13) & 0.0567(12) & -0.0139(10) & 0.0093(9) & 0.0027(10) \\ \text { C22 } & 0.0551(12) & 0.0522(10) & 0.0490(11) & -0.0069(8) & 0.0034(9) & 0.0063(9) \\ \text { C23 } & 0.0789(17) & 0.128(2) & 0.0675(16) & 0.0149(14) & 0.0258(13) & -0.0040(15) \\ \text { N1 } & 0.0431(8) & 0.0391(8) & 0.0486(9) & 0.0023(6) & 0.0071(7) & 0.0041(6) \\ \text { O1 } & 0.0516(8) & 0.0574(8) & 0.0692(9) & -0.0062(7) & 0.0117(6) & 0.0116(6) \\ \text { O2 } & 0.0663(9) & 0.0517(8) & 0.0859(11) & 0.0231(7) & -0.0031(8) & -0.0093(7)\end{array}$

Table 8. Hydrogen atomic coordinates and isotropic atomic displacement parameters $\left(\AA^{2}\right)$ for $3 \mathbf{a b}$.

\begin{tabular}{lllll}
\hline & $\mathbf{x} / \mathbf{a}$ & $\mathbf{y} / \mathbf{b}$ & $\mathbf{z} / \mathbf{c}$ & $\mathbf{U}(\mathbf{e q})$ \\
H3 & 0.9955 & 1.0580 & 0.8611 & 0.08 \\
H4 & 1.2492 & 1.1235 & 0.7950 & 0.093 \\
H5 & 1.5009 & 1.0532 & 0.7327 & 0.091 \\
H6 & 1.4980 & 0.9127 & 0.7317 & 0.076 \\
H10 & 1.2078 & 0.6895 & 0.9360 & 0.057 \\
H11 & 1.0661 & 0.5660 & 0.9649 & 0.062 \\
H13 & 0.5330 & 0.6275 & 0.8482 & 0.056 \\
H14 & 0.6758 & 0.7520 & 0.8208 & 0.051 \\
H15A & 0.4155 & 0.5198 & 0.9121 & 0.119 \\
H15B & 0.4914 & 0.4297 & 0.9142 & 0.119 \\
\hline
\end{tabular}




$\begin{array}{lllll}\text { H15C } & 0.5220 & 0.4827 & 0.8399 & 0.119 \\ \text { H16 } & 0.7800 & 0.9623 & 0.9224 & 0.063 \\ \text { H18 } & 0.8905 & 0.7797 & 1.0188 & 0.063 \\ \text { H19 } & 0.6796 & 0.7167 & 1.1042 & 0.067 \\ \text { H21 } & 0.2154 & 0.8670 & 1.0422 & 0.071 \\ \text { H22 } & 0.4260 & 0.9320 & 0.9587 & 0.062 \\ \text { H23A } & 0.1845 & 0.7800 & 1.1574 & 0.136 \\ \text { H23B } & 0.3692 & 0.7221 & 1.1827 & 0.136 \\ \text { H23C } & 0.2136 & 0.6982 & 1.1132 & 0.136\end{array}$


ylidene)aniline (4ab)

The crystal structure of compound $\mathbf{4 a b}$ has been deposited at the Cambridge Crystallographic Data Centre and allocated the deposition number CCDC: 1963276 (Figure S2).

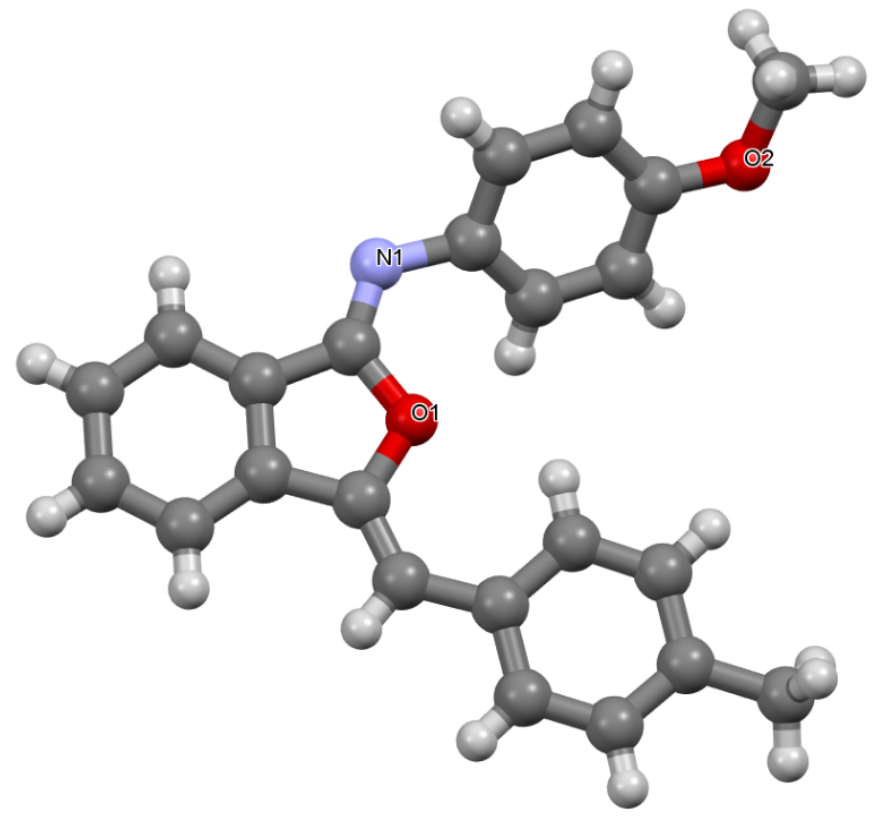

Figure S2. Single crystal X-ray structure of compound 4ab

Table 9. Crystal data and structure refinement for 3a Sample and crystal data for 4 ab.

Identification code

Chemical formula

Formula weight

Temperature

Wavelength

Crystal size
SRM_MH_S2

$\mathrm{C}_{23} \mathrm{H}_{19} \mathrm{NO}_{2}$

$341.39 \mathrm{~g} / \mathrm{mol}$

296(2) K

$0.71073 \AA$

$0.100 \times 0.120 \times 0.250 \mathrm{~mm}$ 
Crystal habit

Crystal system

Space group

Unit cell dimensions

Volume

Z

Density (calculated)

Absorption coefficient

$\mathrm{F}(000)$

Theta range for data collection

Index ranges

Reflections collected

Independent reflections

Coverage of independent reflections

Absorption correction

Max. and min. transmission

Refinement method

Refinement program

Function minimized

Data / restraints / parameters

Goodness-of-fit on $\mathrm{F}^{2}$

Final R indices

Weighting scheme

Absolute structure parameter

Extinction coefficient

Largest diff. peak and hole

R.M.S. deviation from mean clear light colourless Rectangular

orthorhombic

P 212121

$\begin{array}{ll}\mathrm{a}=5.2396(3) \AA & \alpha=90^{\circ} \\ \mathrm{b}=15.5408(9) \AA & \beta=90^{\circ} \\ \mathrm{c}=21.9598(12) \AA & \gamma=90^{\circ}\end{array}$
4

$1.268 \mathrm{~g} / \mathrm{cm}^{3}$

$0.081 \mathrm{~mm}^{-1}$

720

1.61 to $25.00^{\circ}$

$-5<=\mathrm{h}<=6,-18<=\mathrm{k}<=16,-26<=1<=20$

5346

$2944[\mathrm{R}(\mathrm{int})=0.0422]$

$99.6 \%$

multi-scan

0.9920 and 0.9800

Full-matrix least-squares on $\mathrm{F}^{2}$

SHELXL-2014/7 (Sheldrick, 2014)

$\Sigma \mathrm{w}\left(\mathrm{F}_{\mathrm{o}}^{2}-\mathrm{F}_{\mathrm{c}}^{2}\right)^{2}$

2944 / 0 / 238

1.002

1670 data; $\mathrm{I}>2 \sigma(\mathrm{I}) \quad \mathrm{R} 1=0.0533, \mathrm{wR} 2=0.0958$

all data $\mathrm{R} 1=0.1203, \mathrm{wR} 2=0.1263$

where $\mathrm{P}=\left(\mathrm{F}_{\mathrm{o}}^{2}+2 \mathrm{~F}_{\mathrm{c}}{ }^{2}\right) / 3$

$-2.1(10)$

$0.0110(18)$

0.196 and $-0.161 \mathrm{e}^{-3}$

$0.043 \mathrm{e}^{-3}$

Table 10. Atomic coordinates and equivalent isotropic atomic displacement parameters $\left(\AA^{2}\right)$ for 4 ab.

$\mathrm{U}(\mathrm{eq})$ is defined as one third of the trace of the orthogonalized $\mathrm{U}_{\mathrm{ij}}$ tensor.

$\begin{array}{lllll} & \mathbf{x} / \mathbf{a} & \mathbf{y} / \mathbf{b} & \mathbf{z} / \mathbf{c} & \mathbf{U}(\mathbf{e q}) \\ \mathrm{C} 1 & 0.8334(9) & 0.8269(3) & 0.75135(18) & 0.0370(12) \\ \mathrm{C} 2 & 0.0320(9) & 0.8779(3) & 0.78060(19) & 0.0377(13) \\ \mathrm{C} 3 & 0.1933(10) & 0.9404(3) & 0.7571(2) & 0.0512(15) \\ \text { C4 } & 0.3710(10) & 0.9755(4) & 0.7959(2) & 0.0585(16)\end{array}$




\begin{tabular}{lllll} 
& $\mathbf{x} / \mathbf{a}$ & $\mathbf{y} / \mathbf{b}$ & $\mathbf{z} / \mathbf{c}$ & $\mathbf{U}(\mathbf{e q})$ \\
C5 & $0.3887(10)$ & $0.9496(4)$ & $0.8560(2)$ & $0.0587(16)$ \\
C6 & $0.2273(10)$ & $0.8877(4)$ & $0.8795(2)$ & $0.0517(15)$ \\
C7 & $0.0476(9)$ & $0.8527(3)$ & $0.8408(2)$ & $0.0424(14)$ \\
C8 & $0.8560(9)$ & $0.7868(3)$ & $0.8511(2)$ & $0.0409(13)$ \\
C9 & $0.6144(10)$ & $0.6814(3)$ & $0.90237(19)$ & $0.0398(13)$ \\
C10 & $0.4625(10)$ & $0.6759(3)$ & $0.9539(2)$ & $0.0452(14)$ \\
C11 & $0.2831(10)$ & $0.6113(3)$ & $0.9603(2)$ & $0.0488(14)$ \\
C12 & $0.2575(10)$ & $0.5504(3)$ & $0.9149(2)$ & $0.0482(13)$ \\
C13 & $0.4109(10)$ & $0.5541(3)$ & $0.8639(2)$ & $0.0548(15)$ \\
C14 & $0.5870(11)$ & $0.6187(4)$ & $0.8577(2)$ & $0.0528(15)$ \\
C15 & $0.9175(10)$ & $0.4756(4)$ & $0.9656(2)$ & $0.0675(19)$ \\
C16 & $0.7518(9)$ & $0.8262(3)$ & $0.69401(19)$ & $0.0422(13)$ \\
C17 & $0.5556(10)$ & $0.7725(3)$ & $0.6655(2)$ & $0.0423(13)$ \\
C18 & $0.3989(10)$ & $0.7145(3)$ & $0.6962(2)$ & $0.0497(14)$ \\
C19 & $0.2241(10)$ & $0.6644(3)$ & $0.6650(2)$ & $0.0488(14)$ \\
C20 & $0.1959(10)$ & $0.6698(4)$ & $0.6026(2)$ & $0.0504(15)$ \\
C21 & $0.3455(11)$ & $0.7292(4)$ & $0.5730(2)$ & $0.0620(17)$ \\
C22 & $0.5217(11)$ & $0.7799(3)$ & $0.6030(2)$ & $0.0556(16)$ \\
C23 & $0.0065(10)$ & $0.6133(4)$ & $0.5696(2)$ & $0.0714(19)$ \\
N1 & $0.8014(8)$ & $0.7483(3)$ & $0.90036(16)$ & $0.0460(12)$ \\
O1 & $0.7316(6)$ & $0.7725(2)$ & $0.79597(13)$ & $0.0433(9)$ \\
O2 & $0.0897(7)$ & $0.4821(3)$ & $0.91606(16)$ & $0.0678(12)$ \\
\hline & & & &
\end{tabular}

Table 11. Bond lengths ( $\AA$ ) for $4 a b$.

\begin{tabular}{llll}
\hline $\mathrm{C} 1-\mathrm{C} 16$ & $1.330(5)$ & $\mathrm{C} 1-\mathrm{O} 1$ & $1.399(5)$ \\
$\mathrm{C} 1-\mathrm{C} 2$ & $1.458(6)$ & $\mathrm{C} 2-\mathrm{C} 7$ & $1.381(6)$ \\
$\mathrm{C} 2-\mathrm{C} 3$ & $1.387(6)$ & $\mathrm{C} 3-\mathrm{C} 4$ & $1.376(7)$ \\
$\mathrm{C} 3-\mathrm{H} 3$ & 0.93 & $\mathrm{C} 4-\mathrm{C} 5$ & $1.382(6)$ \\
$\mathrm{C} 4-\mathrm{H} 4$ & $\mathrm{C} 5-\mathrm{C} 6$ & $1.381(7)$ \\
$\mathrm{C} 5-\mathrm{H} 5$ & 0.93 & $\mathrm{C} 6-\mathrm{C} 7$ & $1.381(6)$ \\
$\mathrm{C} 6-\mathrm{H} 6$ & 0.93 & $\mathrm{C} 7-\mathrm{C} 8$ & $1.452(7)$ \\
$\mathrm{C} 8-\mathrm{N} 1$ & 0.93 & $\mathrm{C} 8-\mathrm{O} 1$ & $1.394(5)$ \\
$\mathrm{C} 9-\mathrm{C} 10$ & $1.268(5)$ & $\mathrm{C} 9-\mathrm{C} 14$ & $1.391(6)$ \\
$\mathrm{C} 9-\mathrm{N} 1$ & $1.385(6)$ & $\mathrm{C} 10-\mathrm{C} 11$ & $1.383(7)$ \\
$\mathrm{C} 10-\mathrm{H} 10$ & $1.429(6)$ & $\mathrm{C} 11-\mathrm{C} 12$ & $1.381(6)$ \\
$\mathrm{C} 11-\mathrm{H} 11$ & 0.93 & $\mathrm{C} 12-\mathrm{O} 2$ & $1.379(6)$ \\
$\mathrm{C} 12-\mathrm{C} 13$ & 0.93 & $\mathrm{C} 13-\mathrm{C} 14$ & $1.370(7)$ \\
$\mathrm{C} 13-\mathrm{H} 13$ & $1.379(6)$ & $\mathrm{C} 14-\mathrm{H} 14$ & 0.93 \\
$\mathrm{C} 15-\mathrm{O} 2$ & 0.93 & $\mathrm{C} 15-\mathrm{H} 15 \mathrm{~A}$ & 0.96
\end{tabular}




\begin{tabular}{llll}
$\mathrm{C} 15-\mathrm{H} 15 \mathrm{~B}$ & 0.96 & $\mathrm{C} 15-\mathrm{H} 15 \mathrm{C}$ & 0.96 \\
$\mathrm{C} 16-\mathrm{C} 17$ & $1.465(6)$ & $\mathrm{C} 16-\mathrm{H} 16$ & 0.93 \\
$\mathrm{C} 17-\mathrm{C} 22$ & $1.388(6)$ & $\mathrm{C} 17-\mathrm{C} 18$ & $1.394(6)$ \\
$\mathrm{C} 18-\mathrm{C} 19$ & $1.384(6)$ & $\mathrm{C} 18-\mathrm{H} 18$ & 0.93 \\
$\mathrm{C} 19-\mathrm{C} 20$ & $1.381(6)$ & $\mathrm{C} 19-\mathrm{H} 19$ & 0.93 \\
$\mathrm{C} 20-\mathrm{C} 21$ & $1.375(7)$ & $\mathrm{C} 20-\mathrm{C} 23$ & $1.510(7)$ \\
$\mathrm{C} 21-\mathrm{C} 22$ & $1.381(7)$ & $\mathrm{C} 21-\mathrm{H} 21$ & 0.93 \\
$\mathrm{C} 22-\mathrm{H} 22$ & 0.93 & $\mathrm{C} 23-\mathrm{H} 23 \mathrm{~A}$ & 0.96 \\
$\mathrm{C} 23-\mathrm{H} 23 \mathrm{~B}$ & 0.96 & $\mathrm{C} 23-\mathrm{H} 23 \mathrm{C}$ & 0.96 \\
\hline
\end{tabular}

Table 12. Bond angles $\left({ }^{\circ}\right)$ for $4 a b$.

\begin{tabular}{llll}
\hline C16-C1-O1 & $122.4(4)$ & C16-C1-C2 & $130.6(4)$ \\
O1-C1-C2 & $107.0(3)$ & C7-C2-C3 & $121.3(5)$ \\
C7-C2-C1 & $108.0(4)$ & C3-C2-C1 & $130.7(4)$ \\
C4-C3-C2 & $117.3(5)$ & C4-C3-H3 & 121.3 \\
C2-C3-H3 & 121.3 & C3-C4-C5 & $121.4(5)$ \\
C3-C4-H4 & 119.3 & C5-C4-H4 & 119.3 \\
C6-C5-C4 & $121.3(5)$ & C6-C5-H5 & 119.4 \\
C4-C5-H5 & 119.4 & C5-C6-C7 & $117.5(4)$ \\
C5-C6-H6 & 121.3 & C7-C6-H6 & 121.3 \\
C2-C7-C6 & $121.2(5)$ & C2-C7-C8 & $108.0(4)$ \\
C6-C7-C8 & $130.7(5)$ & N1-C8-O1 & $124.1(4)$ \\
N1-C8-C7 & $128.5(5)$ & O1-C8-C7 & $107.4(4)$ \\
C10-C9-C14 & $118.2(5)$ & C10-C9-N1 & $117.6(4)$ \\
C14-C9-N1 & $124.0(4)$ & C11-C10-C9 & $121.2(5)$ \\
C11-C10-H10 & 119.4 & C9-C10-H10 & 119.4 \\
C12-C11-C10 & $119.4(5)$ & C12-C11-H11 & 120.3 \\
C10-C11-H11 & 120.3 & O2-C12-C13 & $114.8(4)$ \\
O2-C12-C11 & $125.2(5)$ & C13-C12-C11 & $120.0(5)$ \\
C14-C13-C12 & $120.3(5)$ & C14-C13-H13 & 119.9 \\
C12-C13-H13 & 119.9 & C13-C14-C9 & $120.9(5)$ \\
C13-C14-H14 & 119.6 & C9-C14-H14 & 119.6 \\
O2-C15-H15A & 109.5 & O2-C15-H15B & 109.5 \\
H15A-C15-H15B & 109.5 & O2-C15-H15C & 109.5 \\
H15A-C15-H15C & 109.5 & H15B-C15-H15C & 109.5 \\
C1-C16-C17 & $129.5(4)$ & C1-C16-H16 & 115.3 \\
C17-C16-H16 & 115.3 & C22-C17-C18 & $117.2(5)$ \\
C22-C17-C16 & $117.8(5)$ & C18-C17-C16 & $125.1(4)$ \\
C19-C18-C17 & $120.9(4)$ & C19-C18-H18 & 119.5 \\
C17-C18-H18 & 119.5 & C20-C19-C18 & $121.9(5)$ \\
C20-C19-H19 & 119.1 & C18-C19-H19 & 19.1
\end{tabular}




\begin{tabular}{llll}
$\mathrm{C} 21-\mathrm{C} 20-\mathrm{C} 19$ & $116.7(5)$ & $\mathrm{C} 21-\mathrm{C} 20-\mathrm{C} 23$ & $122.6(5)$ \\
$\mathrm{C} 19-\mathrm{C} 20-\mathrm{C} 23$ & $120.7(5)$ & $\mathrm{C} 20-\mathrm{C} 21-\mathrm{C} 22$ & $122.6(5)$ \\
$\mathrm{C} 20-\mathrm{C} 21-\mathrm{H} 21$ & 118.7 & $\mathrm{C} 22-\mathrm{C} 21-\mathrm{H} 21$ & 118.7 \\
$\mathrm{C} 21-\mathrm{C} 22-\mathrm{C} 17$ & $120.7(5)$ & $\mathrm{C} 21-\mathrm{C} 22-\mathrm{H} 22$ & 119.7 \\
$\mathrm{C} 17-\mathrm{C} 22-\mathrm{H} 22$ & 119.7 & $\mathrm{C} 20-\mathrm{C} 23-\mathrm{H} 23 \mathrm{~A}$ & 109.5 \\
$\mathrm{C} 20-\mathrm{C} 23-\mathrm{H} 23 \mathrm{~B}$ & 109.5 & $\mathrm{H} 23 \mathrm{~A}-\mathrm{C} 23-\mathrm{H} 23 \mathrm{~B}$ & 109.5 \\
$\mathrm{C} 20-\mathrm{C} 23-\mathrm{H} 23 \mathrm{C}$ & 109.5 & $\mathrm{H} 23 \mathrm{~A}-\mathrm{C} 23-\mathrm{H} 23 \mathrm{C}$ & 109.5 \\
$\mathrm{H} 23 \mathrm{~B}-\mathrm{C} 23-\mathrm{H} 23 \mathrm{C}$ & 109.5 & $\mathrm{C} 8-\mathrm{N} 1-\mathrm{C} 9$ & $121.6(4)$ \\
$\mathrm{C} 8-\mathrm{O} 1-\mathrm{C} 1$ & $109.5(4)$ & $\mathrm{C} 12-\mathrm{O} 2-\mathrm{C} 15$ & $118.4(4)$ \\
\hline
\end{tabular}

Table 13. Torsion angles $\left(^{\circ}\right.$ ) for 4 ab.

\begin{tabular}{|c|c|c|c|}
\hline $\mathrm{C} 16-\mathrm{C} 1-\mathrm{C} 2-\mathrm{C} 7$ & $-178.4(5)$ & $\mathrm{O} 1-\mathrm{C} 1-\mathrm{C} 2-\mathrm{C} 7$ & $0.9(5)$ \\
\hline $\mathrm{C} 16-\mathrm{C} 1-\mathrm{C} 2-\mathrm{C} 3$ & $-0.1(9)$ & $\mathrm{O} 1-\mathrm{C} 1-\mathrm{C} 2-\mathrm{C} 3$ & $179.2(5)$ \\
\hline $\mathrm{C} 7-\mathrm{C} 2-\mathrm{C} 3-\mathrm{C} 4$ & $0.7(8)$ & $\mathrm{C} 1-\mathrm{C} 2-\mathrm{C} 3-\mathrm{C} 4$ & $-177.4(5)$ \\
\hline C2-C3-C4-C5 & $0.0(8)$ & C3-C4-C5-C6 & $-0.4(9)$ \\
\hline C4-C5-C6-C7 & $0.0(8)$ & $\mathrm{C} 3-\mathrm{C} 2-\mathrm{C} 7-\mathrm{C} 6$ & $-1.1(8)$ \\
\hline $\mathrm{C} 1-\mathrm{C} 2-\mathrm{C} 7-\mathrm{C} 6$ & $177.4(5)$ & $\mathrm{C} 3-\mathrm{C} 2-\mathrm{C} 7-\mathrm{C} 8$ & $-179.8(5)$ \\
\hline $\mathrm{C} 1-\mathrm{C} 2-\mathrm{C} 7-\mathrm{C} 8$ & $-1.3(5)$ & C5-C6-C7-C2 & $0.7(8)$ \\
\hline C5-C6-C7-C8 & $179.1(5)$ & C2-C7-C8-N1 & $-177.8(5)$ \\
\hline C6-C7-C8-N1 & $3.6(9)$ & C2-C7-C8-O1 & $1.2(5)$ \\
\hline C6-C7-C8-O1 & $-177.3(5)$ & C14-C9-C10-C11 & $-1.7(8)$ \\
\hline $\mathrm{N} 1-\mathrm{C} 9-\mathrm{C} 10-\mathrm{C} 11$ & $-177.8(5)$ & C9-C10-C11-C12 & $1.0(8)$ \\
\hline $\mathrm{C} 10-\mathrm{C} 11-\mathrm{C} 12-\mathrm{O} 2$ & $178.9(5)$ & C10-C11-C12-C13 & $0.3(8)$ \\
\hline $\mathrm{O} 2-\mathrm{C} 12-\mathrm{C} 13-\mathrm{C} 14$ & $-179.5(5)$ & C11-C12-C13-C14 & $-0.8(8)$ \\
\hline C12-C13-C14-C9 & $0.0(9)$ & C10-C9-C14-C13 & $1.3(8)$ \\
\hline N1-C9-C14-C13 & $177.0(5)$ & $\mathrm{O} 1-\mathrm{C} 1-\mathrm{C} 16-\mathrm{C} 17$ & $-1.0(8)$ \\
\hline C2-C1-C16-C17 & $178.3(5)$ & C1-C16-C17-C22 & $-174.7(5)$ \\
\hline C1-C16-C17-C18 & $5.6(8)$ & C22-C17-C18-C19 & $2.4(8)$ \\
\hline C16-C17-C18-C19 & $-177.9(5)$ & C17-C18-C19-C20 & $-0.4(8)$ \\
\hline C18-C19-C20-C21 & $-1.7(8)$ & C18-C19-C20-C23 & $179.0(5)$ \\
\hline C19-C20-C21-C22 & $1.8(8)$ & C23-C20-C21-C22 & $-178.9(5)$ \\
\hline $\mathrm{C} 20-\mathrm{C} 21-\mathrm{C} 22-\mathrm{C} 17$ & $0.2(9)$ & C18-C17-C22-C21 & $-2.3(8)$ \\
\hline C16-C17-C22-C21 & $178.0(5)$ & O1-C8-N1-C9 & $4.3(8)$ \\
\hline C7-C8-N1-C9 & $-176.8(5)$ & C10-C9-N1-C8 & $-144.1(5)$ \\
\hline C14-C9-N1-C8 & $40.1(8)$ & N1-C8-O1-C1 & $178.4(5)$ \\
\hline C7-C8-O1-C1 & $-0.7(5)$ & C16-C1-O1-C8 & $179.3(4)$ \\
\hline $\mathrm{C} 2-\mathrm{C} 1-\mathrm{O} 1-\mathrm{C} 8$ & $-0.1(5)$ & C13-C12-O2-C15 & $-177.3(4)$ \\
\hline C11-C12-O2-C15 & $4.0(8)$ & & \\
\hline
\end{tabular}




\begin{tabular}{|c|c|c|c|c|c|c|}
\hline \multicolumn{6}{|c|}{$\begin{array}{l}\text { The anisotropic atomic displacement factor exponent takes the form: }-2 \pi^{2}\left[\mathrm{~h}^{2} \mathrm{a}^{* 2} \mathrm{U}_{11}+\right. \\
\left.\ldots+2 \mathrm{~h} \mathrm{k} \mathrm{a}{ }^{*} \mathrm{~b}^{*} \mathrm{U}_{12}\right]\end{array}$} & \multirow[b]{2}{*}{$\mathbf{U}_{12}$} \\
\hline & $\mathrm{U}_{11}$ & $\mathbf{U}_{22}$ & $\mathbf{U}_{33}$ & $\mathbf{U}_{23}$ & $\mathbf{U}_{13}$ & \\
\hline $\mathrm{C} 1$ & $0.042(3)$ & $0.036(3)$ & $0.033(2)$ & $0.003(2)$ & $0.006(3)$ & $-0.001(3)$ \\
\hline $\mathrm{C} 2$ & $0.038(3)$ & $0.037(3)$ & $0.039(3)$ & $-0.003(2)$ & $0.007(3)$ & $-0.004(3)$ \\
\hline $\mathrm{C} 3$ & $0.060(4)$ & $0.048(4)$ & $0.046(3)$ & $0.000(3)$ & $0.012(3)$ & $-0.006(3)$ \\
\hline $\mathrm{C} 4$ & $0.054(4)$ & $0.059(4)$ & $0.063(3)$ & $-0.005(3)$ & $0.010(3)$ & $-0.018(3)$ \\
\hline $\mathrm{C} 5$ & $0.051(4)$ & $0.068(5)$ & $0.058(4)$ & $-0.015(3)$ & $0.000(3)$ & $-0.016(3)$ \\
\hline C6 & $0.053(4)$ & $0.059(4)$ & $0.043(3)$ & $-0.006(3)$ & $0.001(3)$ & $-0.006(3)$ \\
\hline $\mathrm{C} 7$ & $0.041(3)$ & $0.046(4)$ & $0.040(3)$ & $-0.006(3)$ & $-0.001(3)$ & $-0.005(3)$ \\
\hline $\mathrm{C} 8$ & $0.042(3)$ & $0.048(4)$ & $0.033(3)$ & $-0.007(2)$ & $-0.004(3)$ & $-0.003(3)$ \\
\hline C9 & $0.050(3)$ & $0.040(3)$ & $0.029(3)$ & $0.002(2)$ & $0.003(3)$ & $-0.003(3)$ \\
\hline $\mathrm{C} 10$ & $0.058(4)$ & $0.044(4)$ & $0.034(3)$ & $-0.003(3)$ & $0.004(3)$ & $0.002(3)$ \\
\hline $\mathrm{C} 11$ & $0.061(4)$ & $0.047(4)$ & $0.039(3)$ & $0.003(3)$ & $0.017(3)$ & $0.003(3)$ \\
\hline $\mathrm{C} 12$ & $0.050(3)$ & $0.046(4)$ & $0.048(3)$ & $-0.002(3)$ & $0.010(3)$ & $-0.006(3)$ \\
\hline $\mathrm{C} 13$ & $0.076(4)$ & $0.049(4)$ & $0.039(3)$ & $-0.011(3)$ & $0.019(3)$ & $-0.016(3)$ \\
\hline $\mathrm{C} 14$ & $0.068(4)$ & $0.051(4)$ & $0.040(3)$ & $-0.002(3)$ & $0.018(3)$ & $-0.013(3)$ \\
\hline $\mathrm{C} 15$ & $0.067(4)$ & $0.069(5)$ & $0.067(3)$ & $0.010(3)$ & $0.030(3)$ & $-0.016(4)$ \\
\hline $\mathrm{C} 16$ & $0.050(3)$ & $0.039(3)$ & $0.037(3)$ & $0.005(2)$ & $0.000(3)$ & $-0.004(3)$ \\
\hline $\mathrm{C} 17$ & $0.047(3)$ & $0.042(4)$ & $0.038(3)$ & $0.003(2)$ & $0.001(3)$ & $0.002(3)$ \\
\hline $\mathrm{C} 18$ & $0.050(3)$ & $0.055(4)$ & $0.045(3)$ & $0.002(3)$ & $-0.003(3)$ & $-0.006(3)$ \\
\hline C19 & $0.046(4)$ & $0.046(4)$ & $0.054(3)$ & $0.006(3)$ & $-0.002(3)$ & $-0.008(3)$ \\
\hline $\mathrm{C} 20$ & $0.051(4)$ & $0.047(4)$ & $0.054(3)$ & $-0.001(3)$ & $-0.010(3)$ & $-0.003(3)$ \\
\hline $\mathrm{C} 21$ & $0.071(4)$ & $0.071(5)$ & $0.045(3)$ & $0.006(3)$ & $-0.013(3)$ & $-0.004(4)$ \\
\hline $\mathrm{C} 22$ & $0.070(4)$ & $0.055(4)$ & $0.042(3)$ & $0.009(3)$ & $-0.010(3)$ & $-0.014(3)$ \\
\hline $\mathrm{C} 23$ & $0.066(4)$ & $0.075(5)$ & $0.073(4)$ & $-0.007(3)$ & $-0.021(3)$ & $-0.006(4)$ \\
\hline N1 & $0.058(3)$ & $0.046(3)$ & $0.034(2)$ & $-0.002(2)$ & $-0.001(2)$ & $-0.009(2)$ \\
\hline $\mathrm{O} 1$ & $0.052(2)$ & $0.043(2)$ & $0.0344(16)$ & $0.0030(16)$ & $-0.0052(17)$ & $-0.0158(18)$ \\
\hline $\mathrm{O} 2$ & $0.072(3)$ & $0.060(3)$ & $0.071(2)$ & $-0.013(2)$ & $0.031(2)$ & $-0.024(2)$ \\
\hline
\end{tabular}

Table 15. Hydrogen atomic coordinates and isotropic atomic displacement parameters $\left(\AA^{2}\right)$ for $4 a b$.

$\begin{array}{lllll} & \mathbf{x} / \mathbf{a} & \mathbf{y} / \mathbf{b} & \mathbf{z} / \mathbf{c} & \mathbf{U}(\mathbf{e q}) \\ \mathrm{H} 3 & 1.1817 & 0.9579 & 0.7167 & 0.061 \\ \mathrm{H} 4 & 1.4817 & 1.0176 & 0.7815 & 0.07 \\ \mathrm{H} 5 & 1.5117 & 0.9743 & 0.8810 & 0.07 \\ \text { H6 } & 1.2392 & 0.8703 & 0.9199 & 0.062 \\ \text { H10 } & 0.4815 & 0.7165 & 0.9847 & 0.054 \\ \text { H11 } & 0.1807 & 0.6089 & 0.9948 & 0.059\end{array}$




\begin{tabular}{lllll} 
& $\mathbf{x} / \mathbf{a}$ & $\mathbf{y} / \mathbf{b}$ & $\mathbf{z} / \mathbf{c}$ & $\mathbf{U}(\mathbf{e q})$ \\
H13 & 0.3947 & 0.5126 & 0.8337 & 0.066 \\
H14 & 0.6895 & 0.6206 & 0.8232 & 0.063 \\
H15A & -0.1851 & 0.5267 & 0.9676 & 0.101 \\
H15B & 0.0117 & 0.4692 & 1.0028 & 0.101 \\
H15C & -0.1908 & 0.4264 & 0.9599 & 0.101 \\
H16 & 0.8308 & 0.8655 & 0.6683 & 0.051 \\
H18 & 0.4120 & 0.7094 & 0.7383 & 0.06 \\
H19 & 0.1227 & 0.6260 & 0.6867 & 0.059 \\
H21 & 0.3274 & 0.7355 & 0.5311 & 0.074 \\
H22 & 0.6185 & 0.8194 & 0.5811 & 0.067 \\
H23A & 0.0406 & 0.6149 & 0.5267 & 0.107 \\
H23B & -0.1634 & 0.6338 & 0.5771 & 0.107 \\
H23C & 0.0219 & 0.5552 & 0.5840 & 0.107 \\
\hline
\end{tabular}



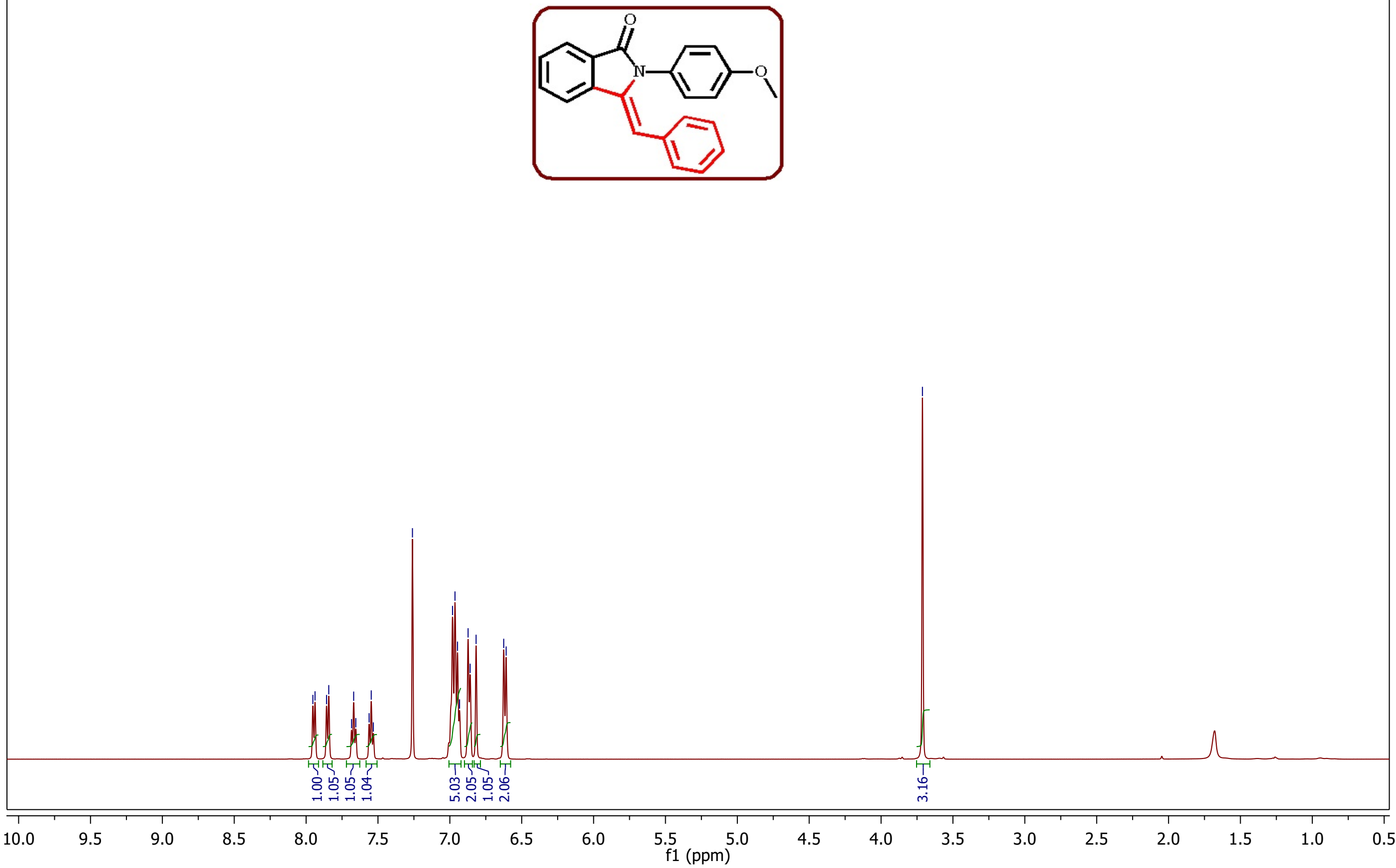






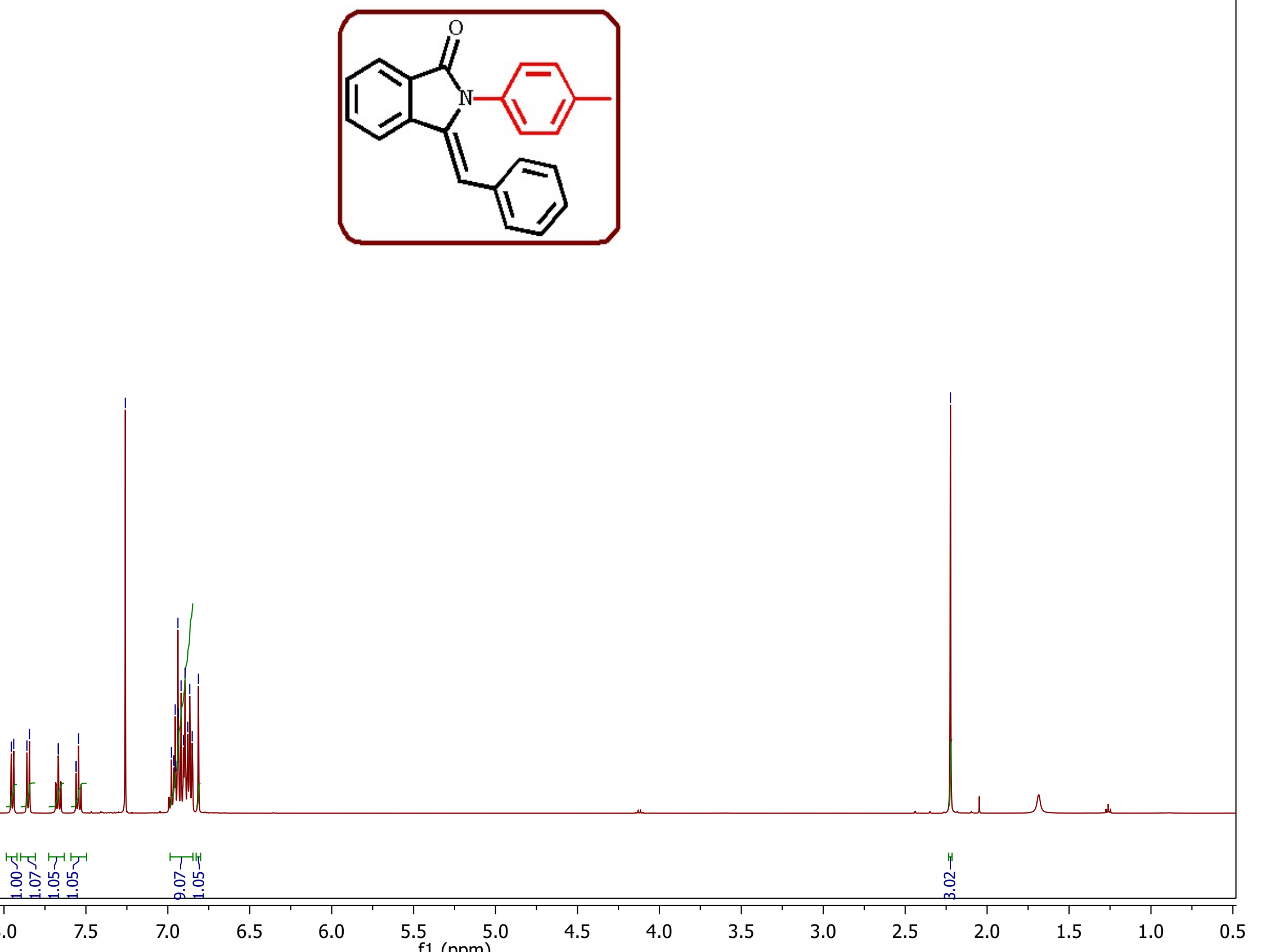

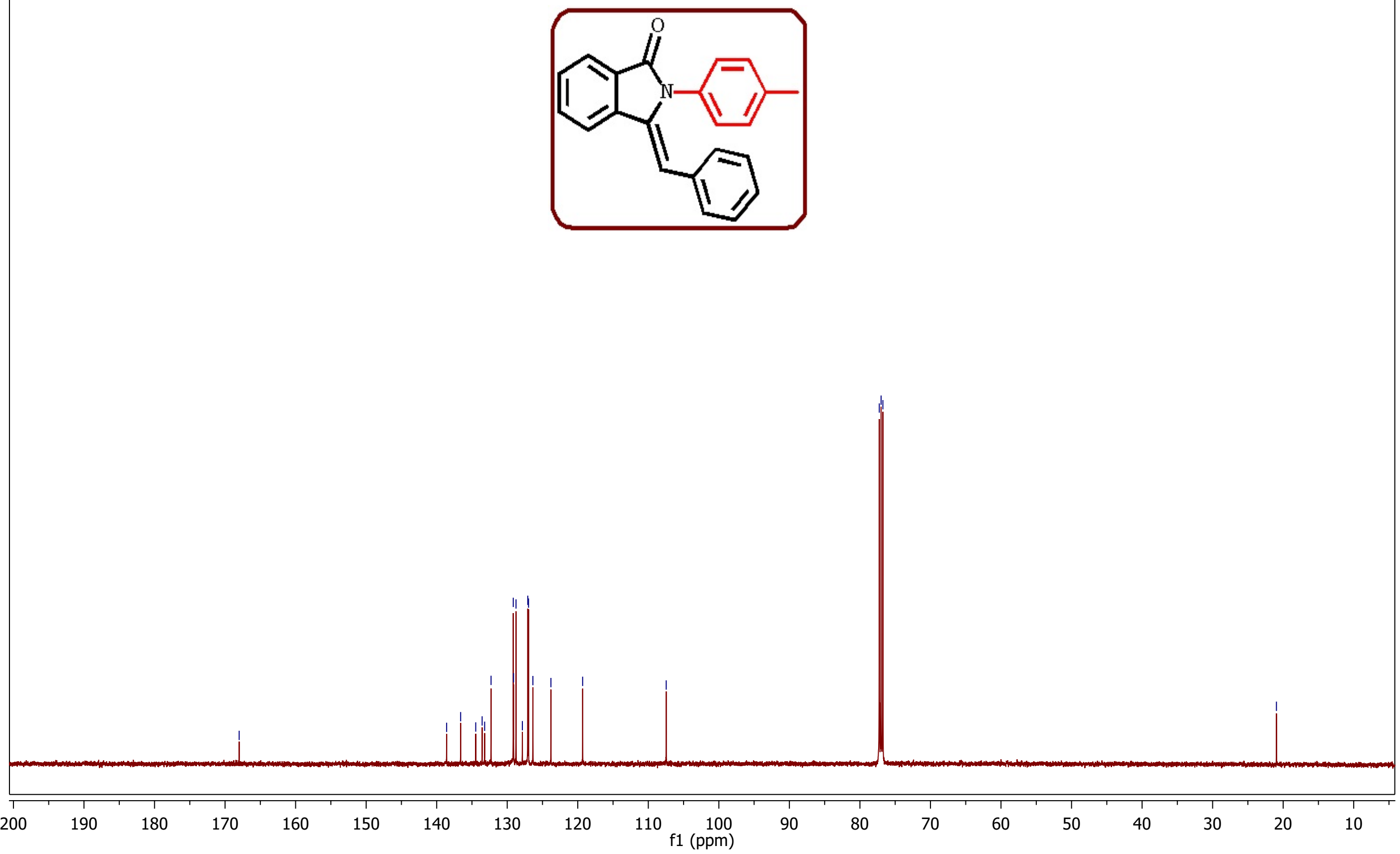

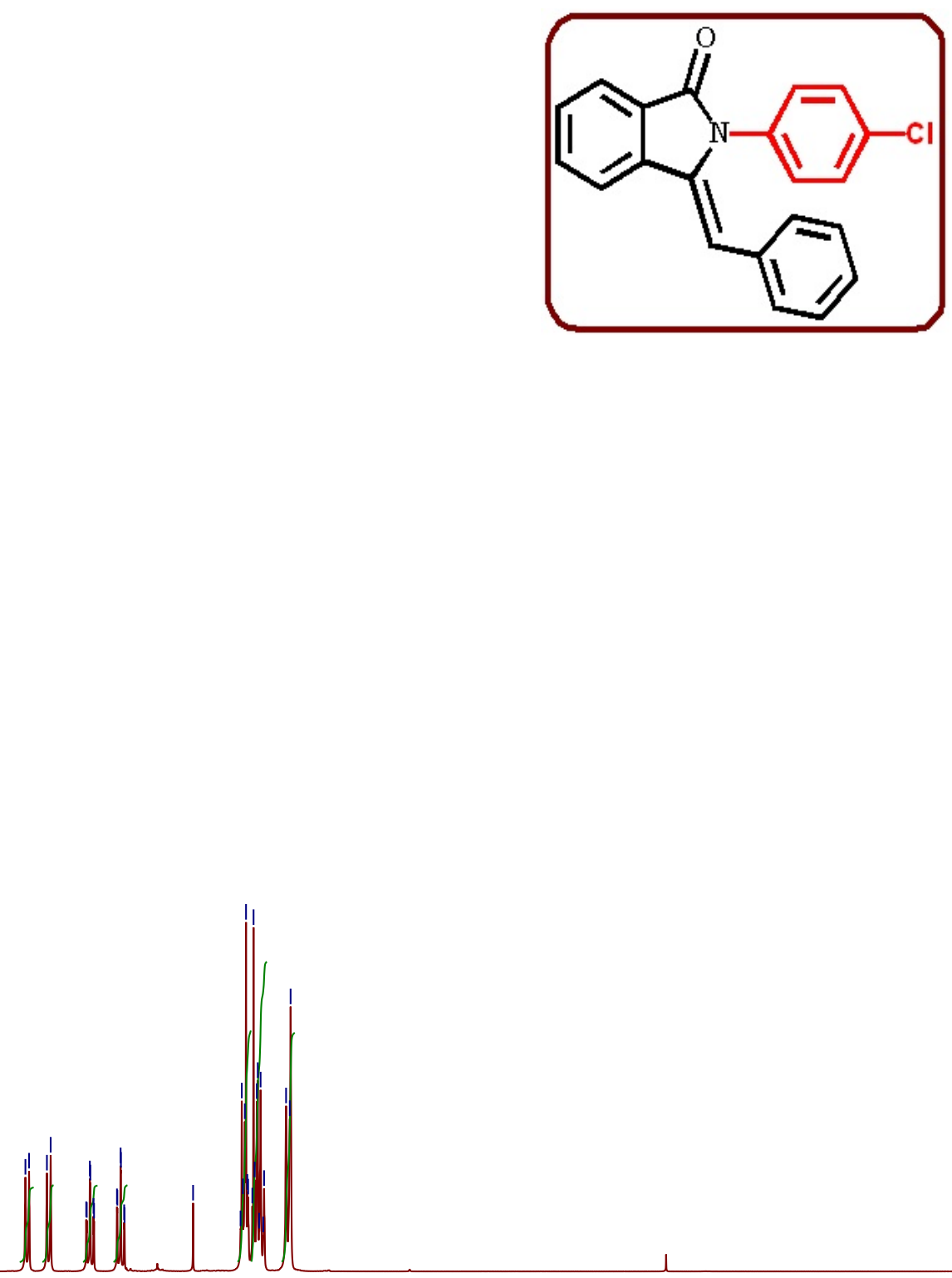


ơ ग्र

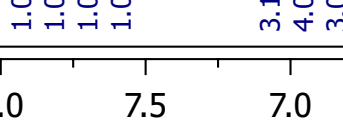




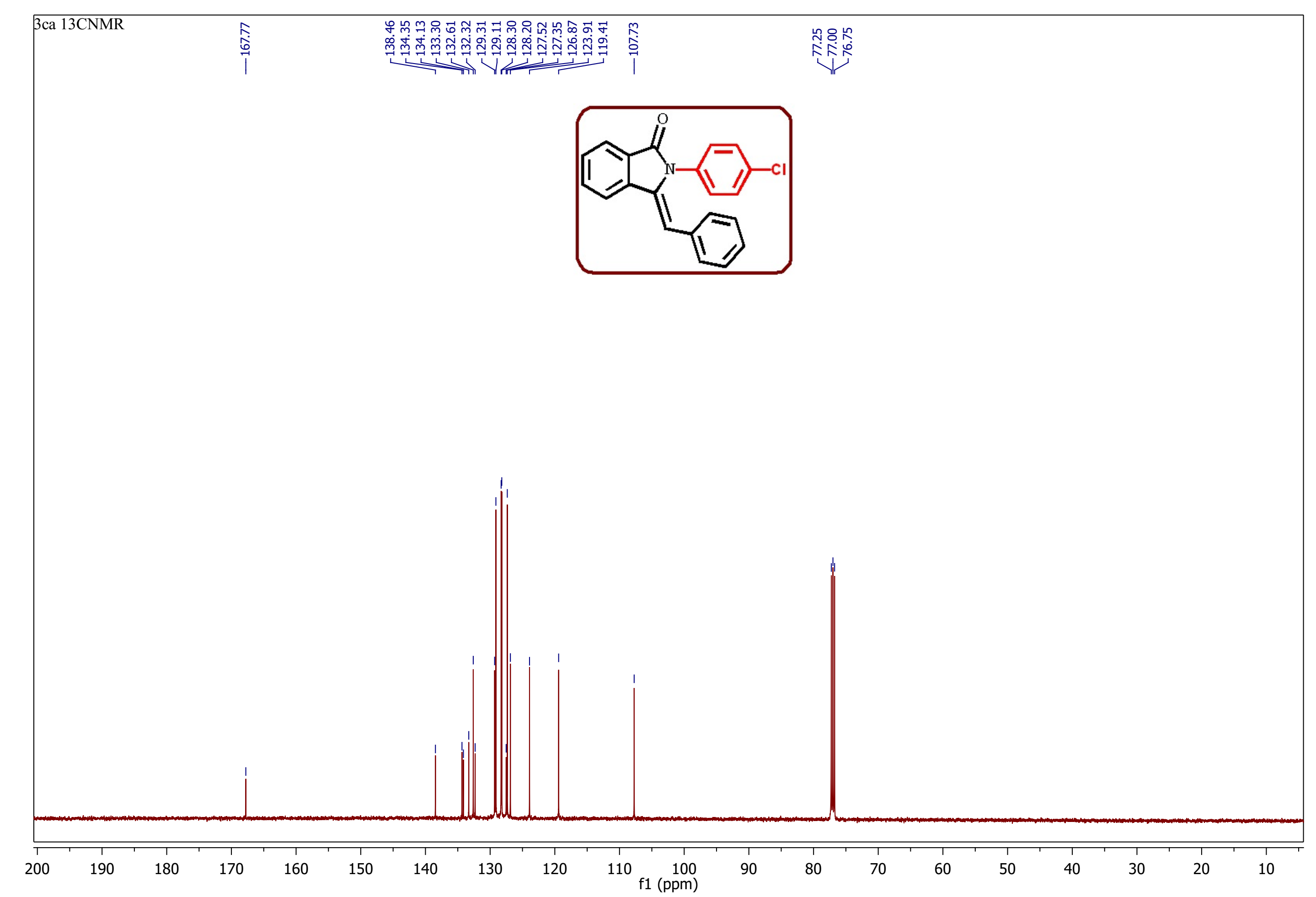










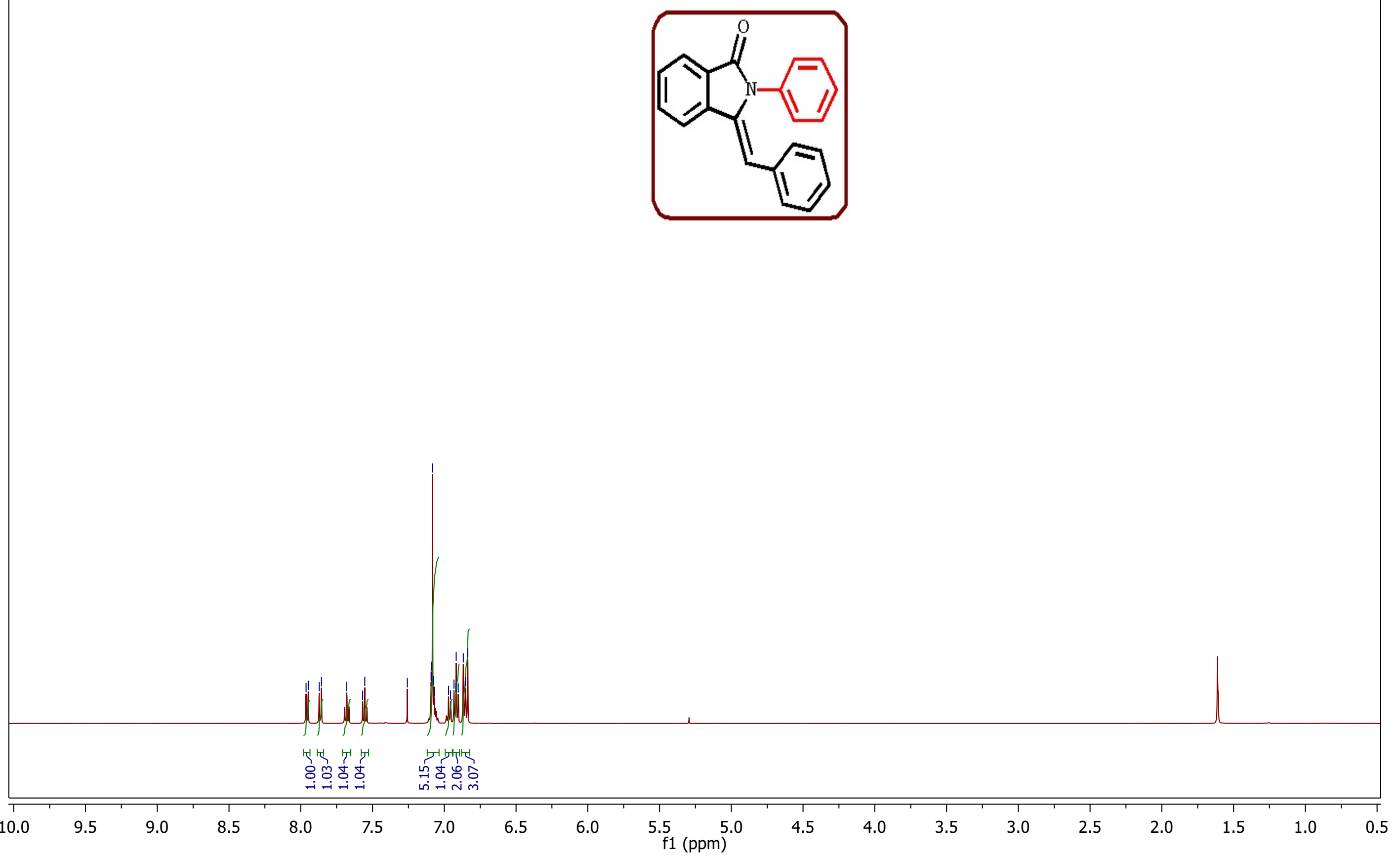

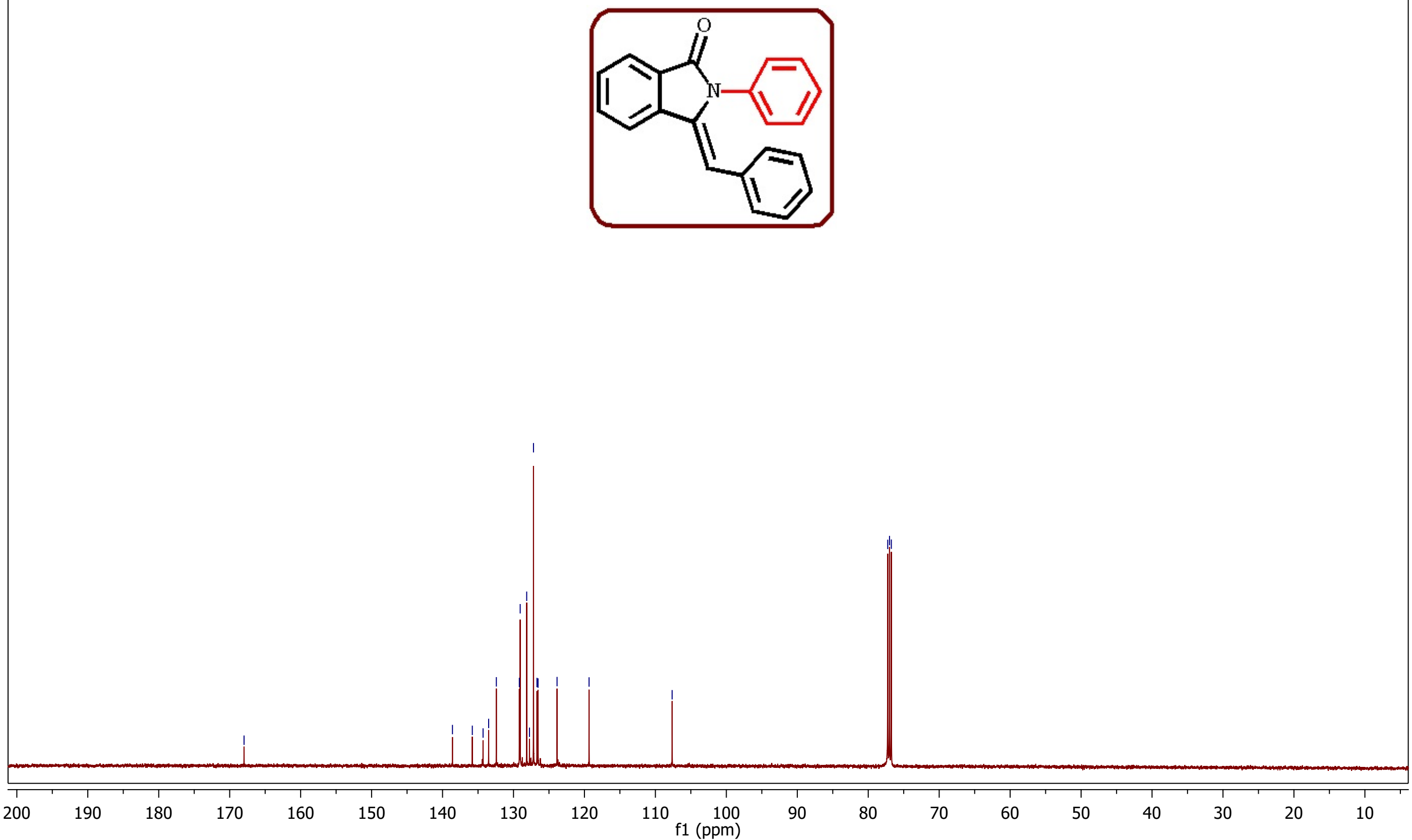




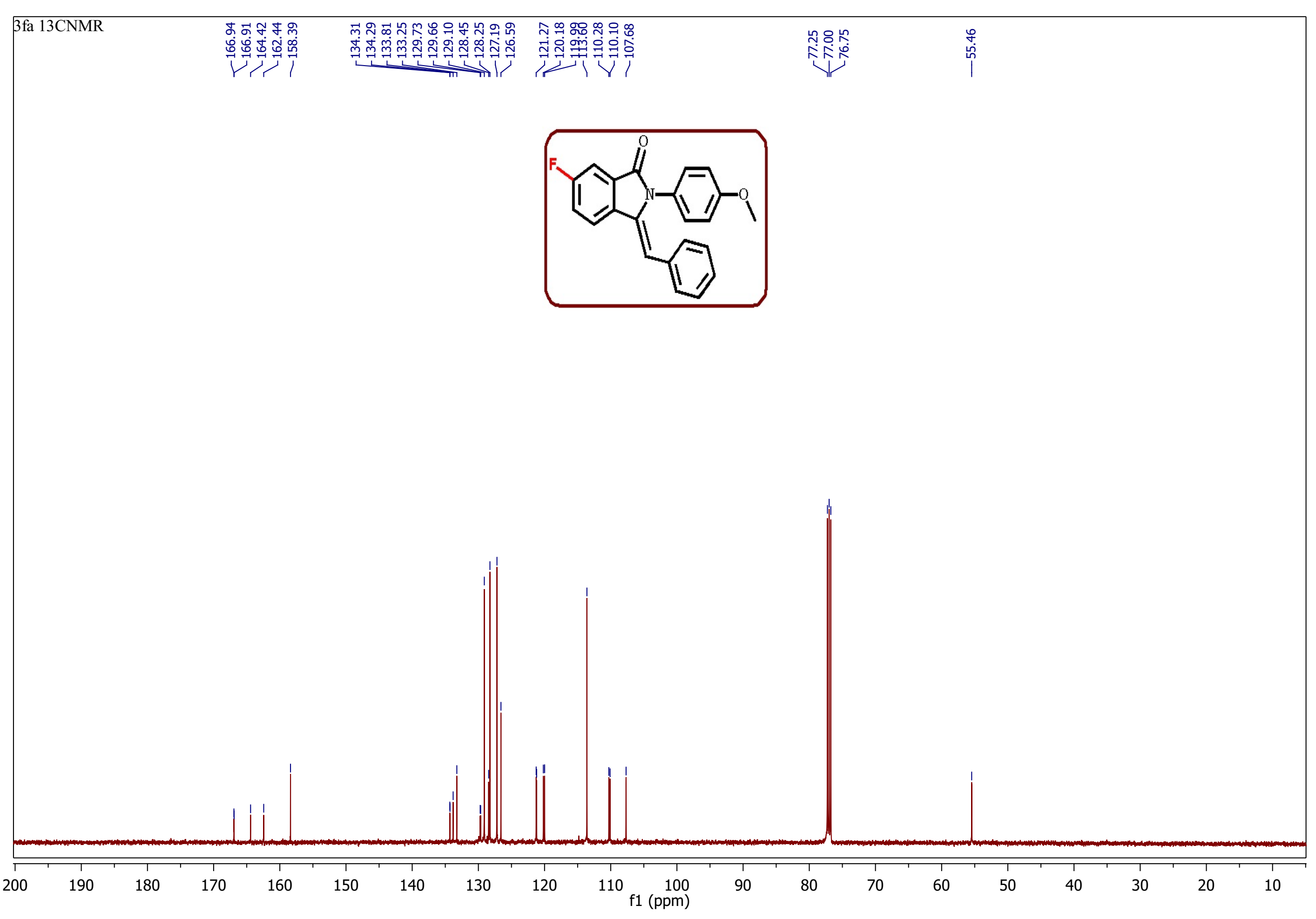



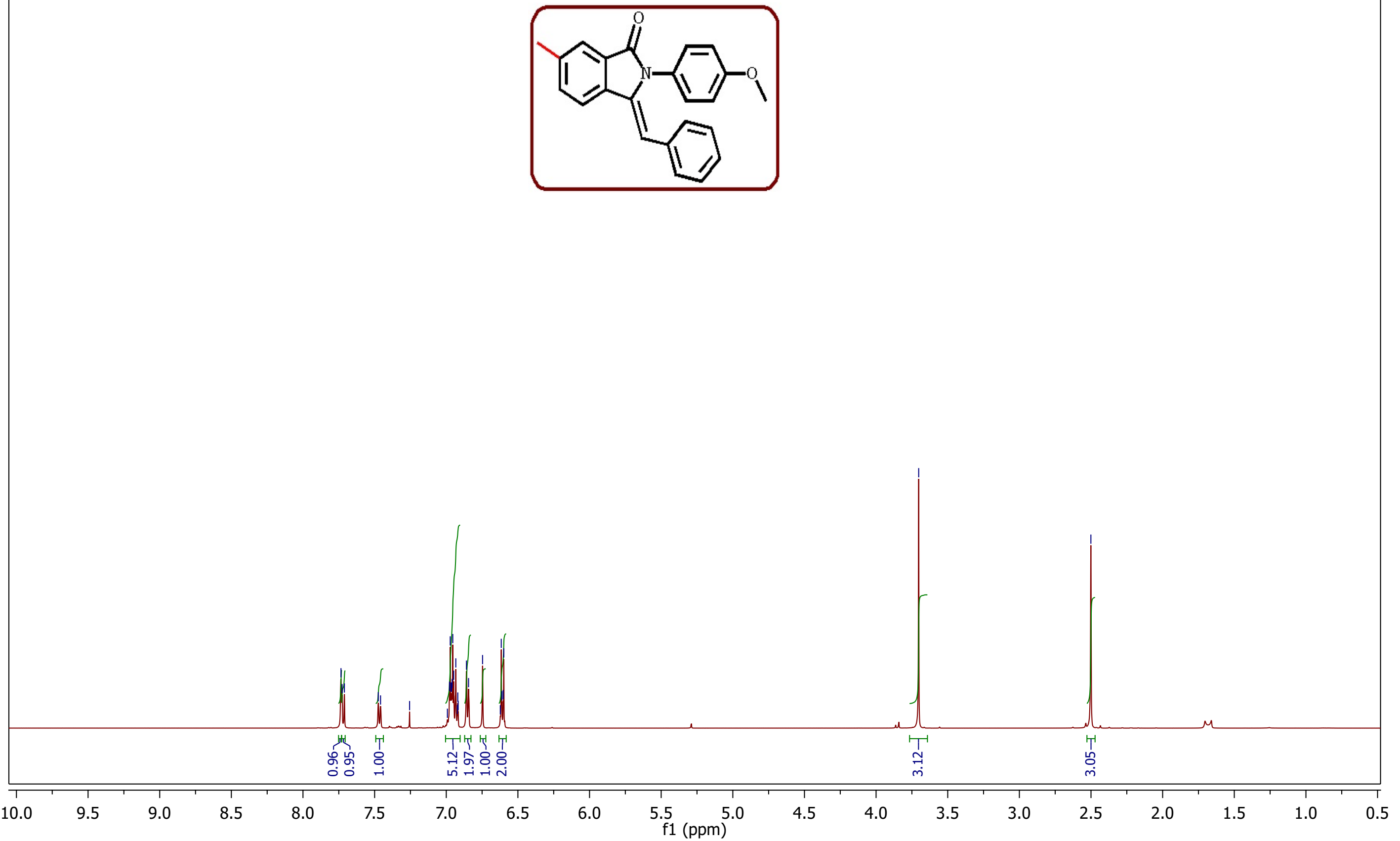

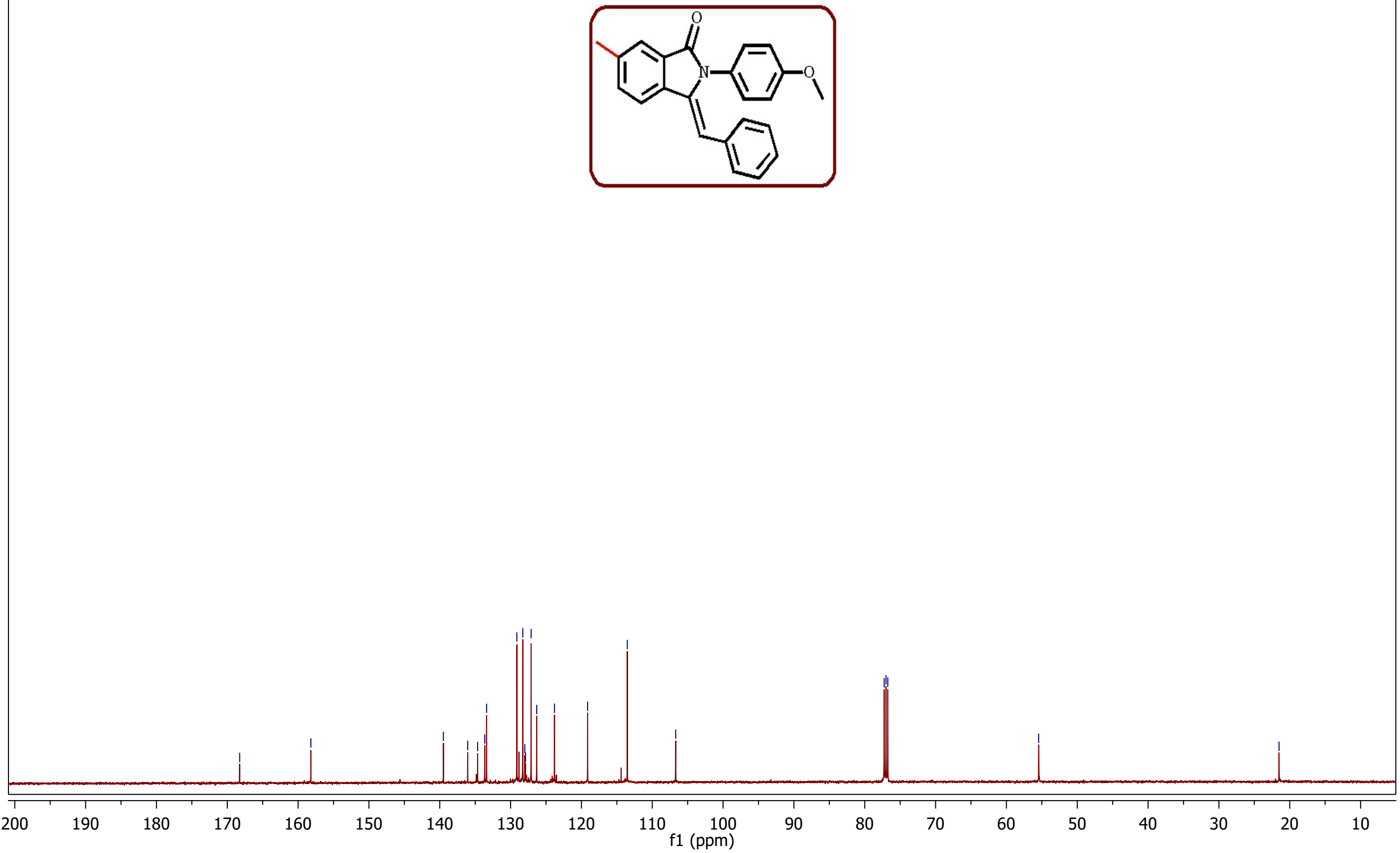

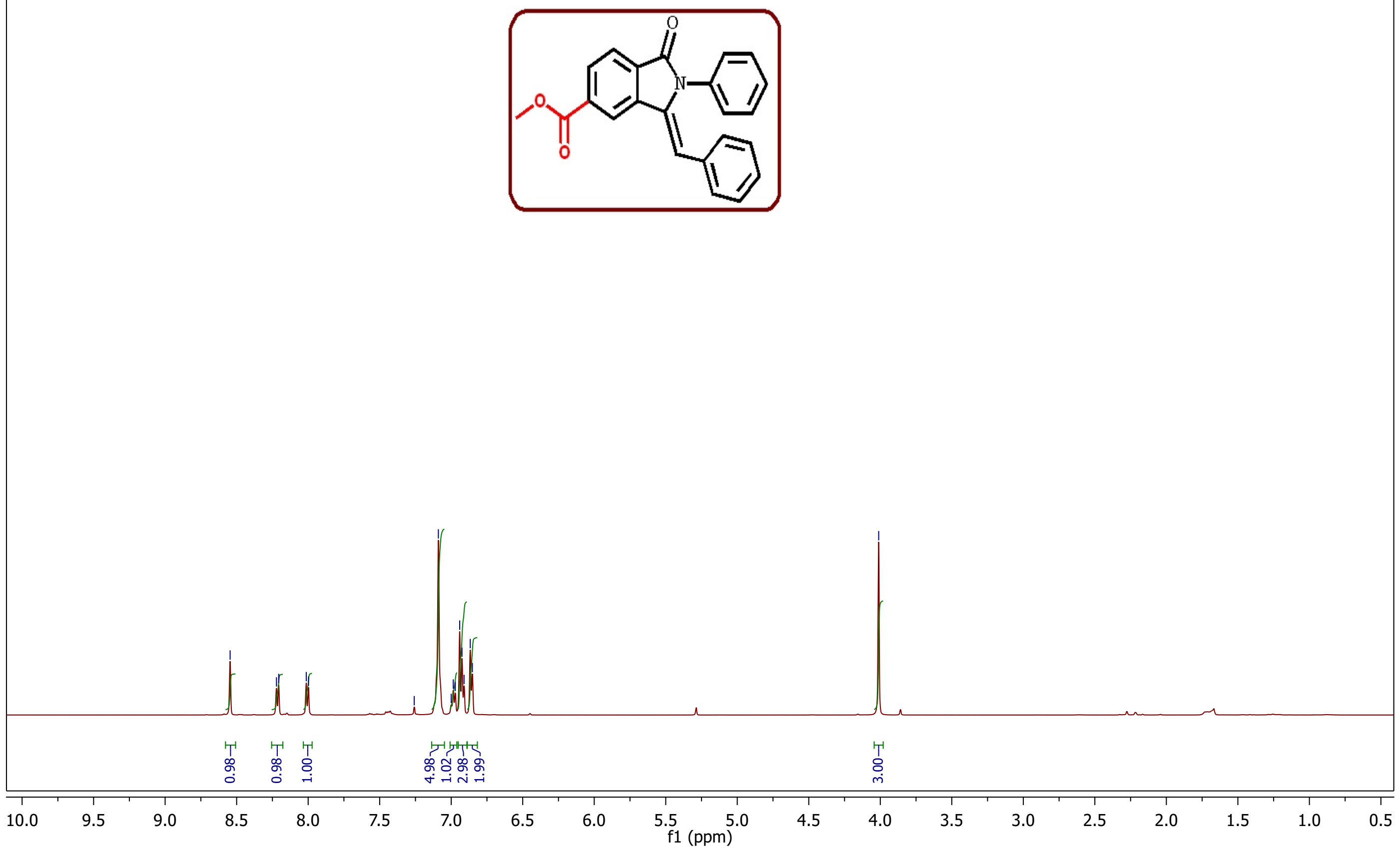

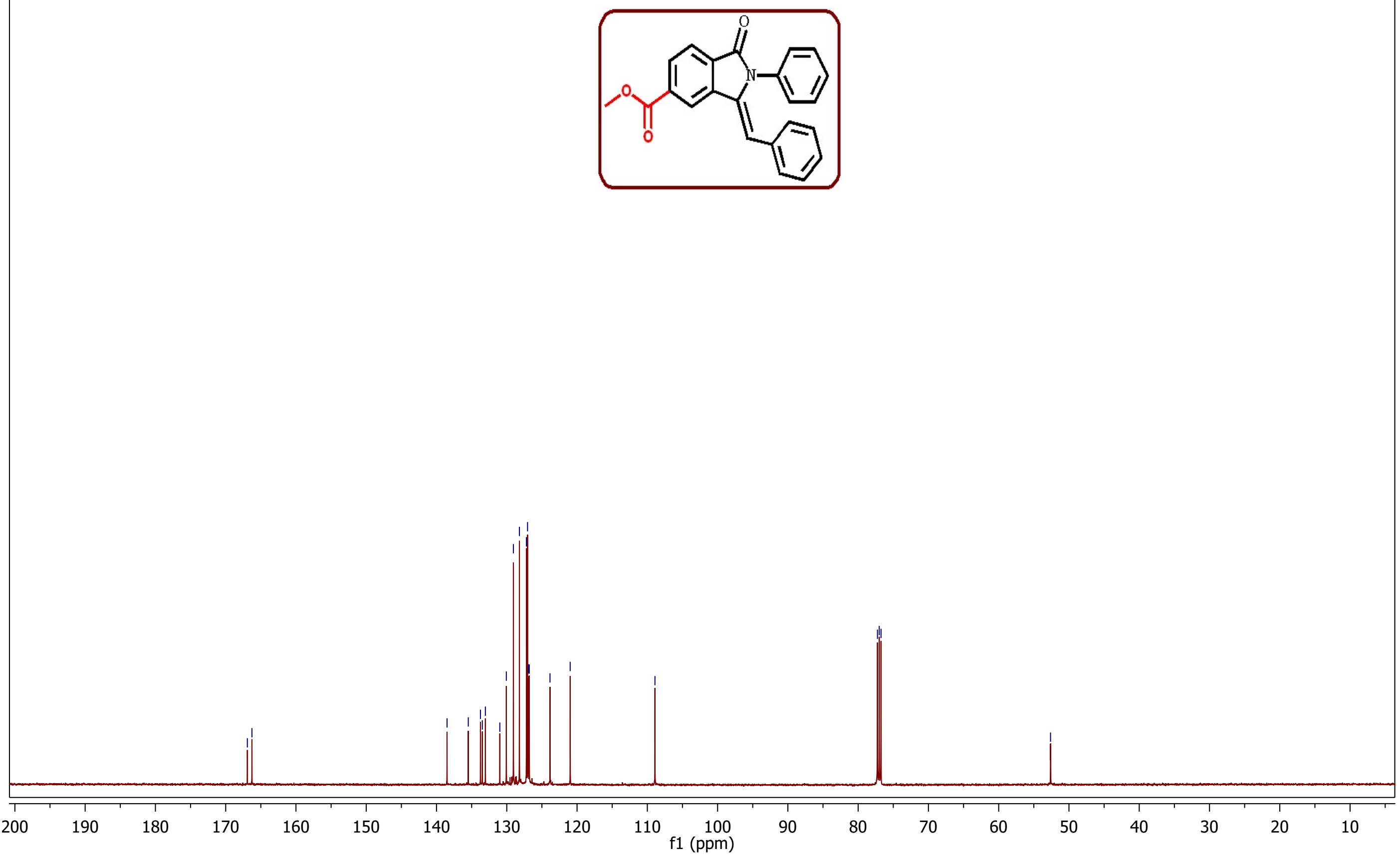


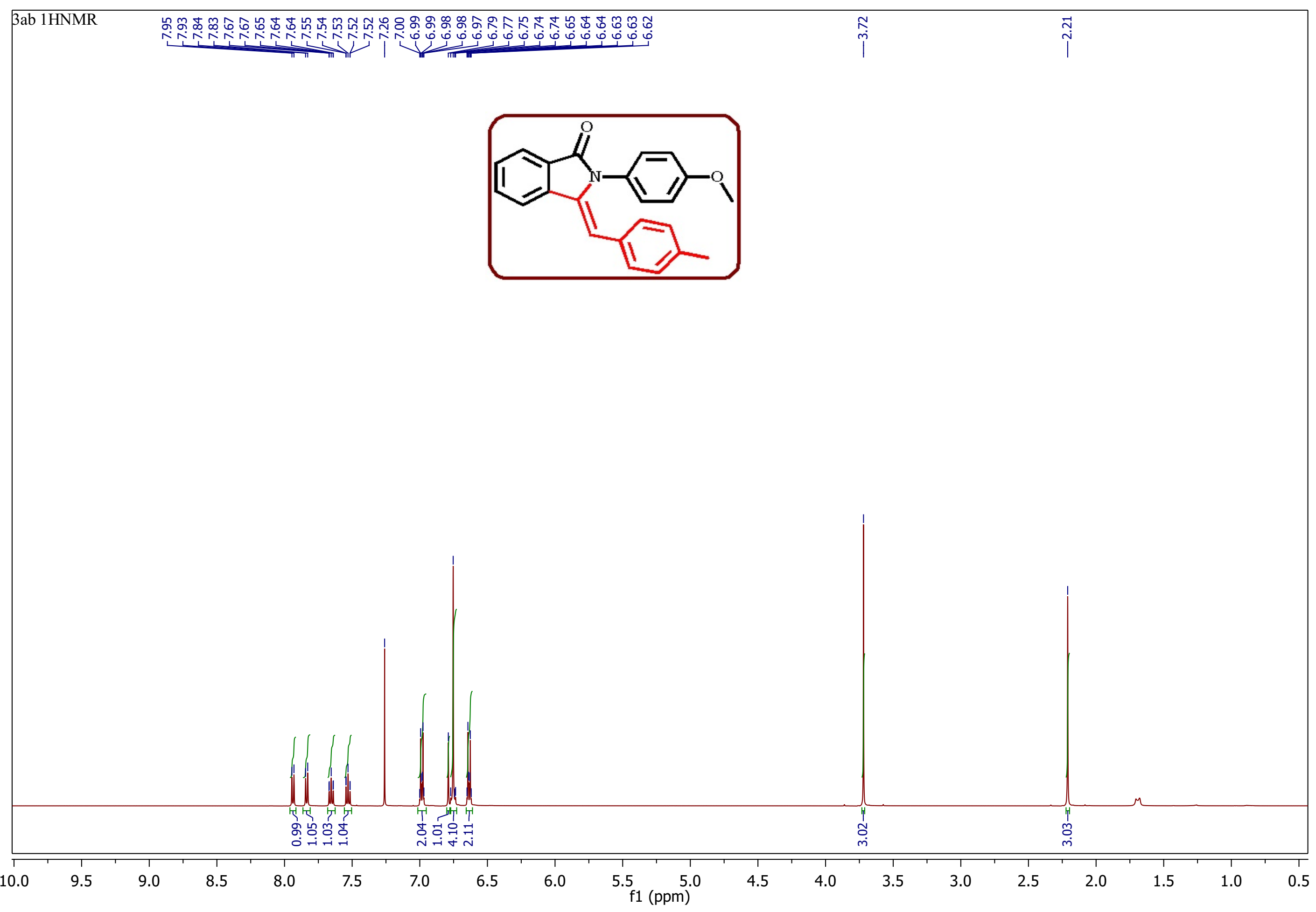




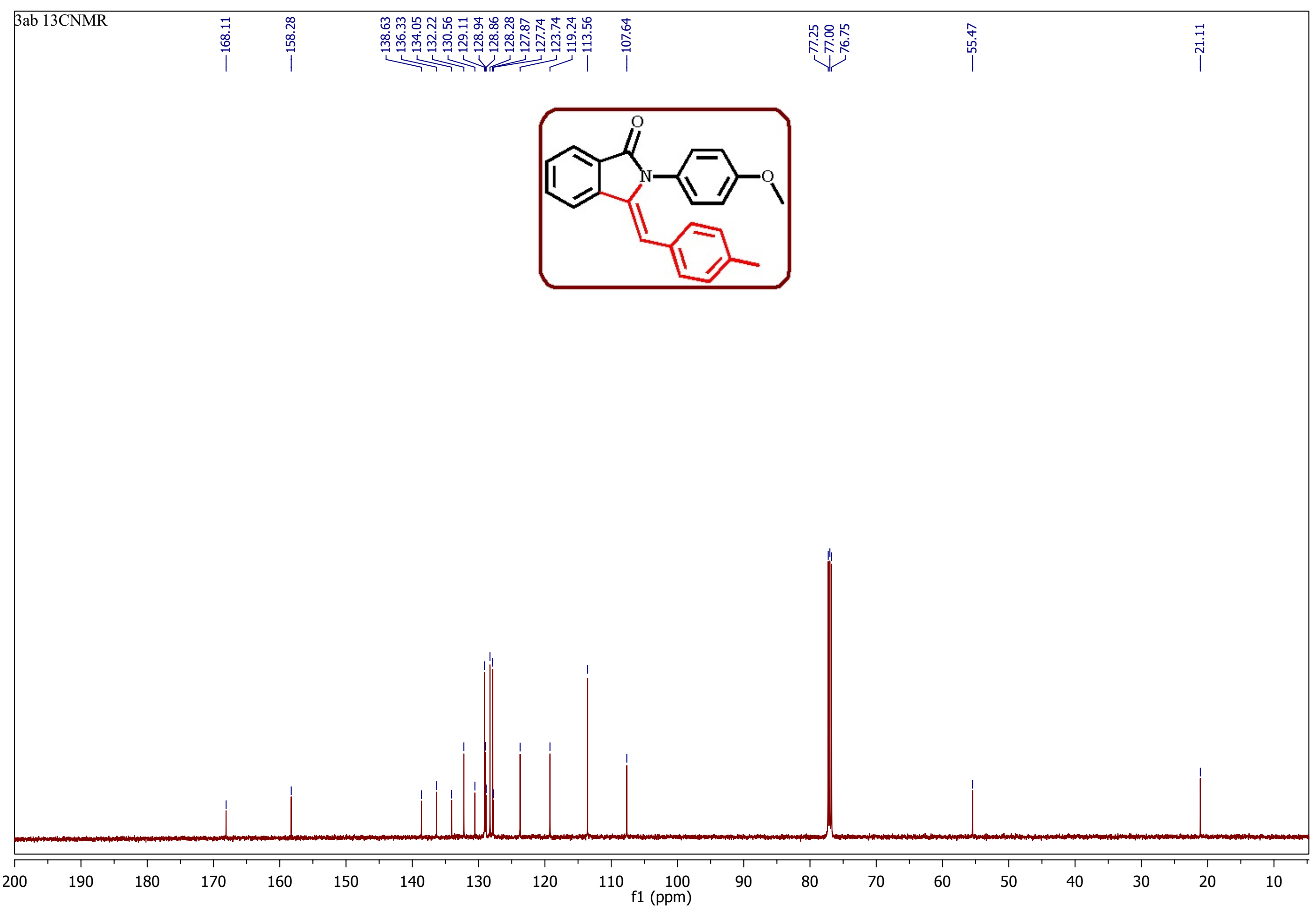



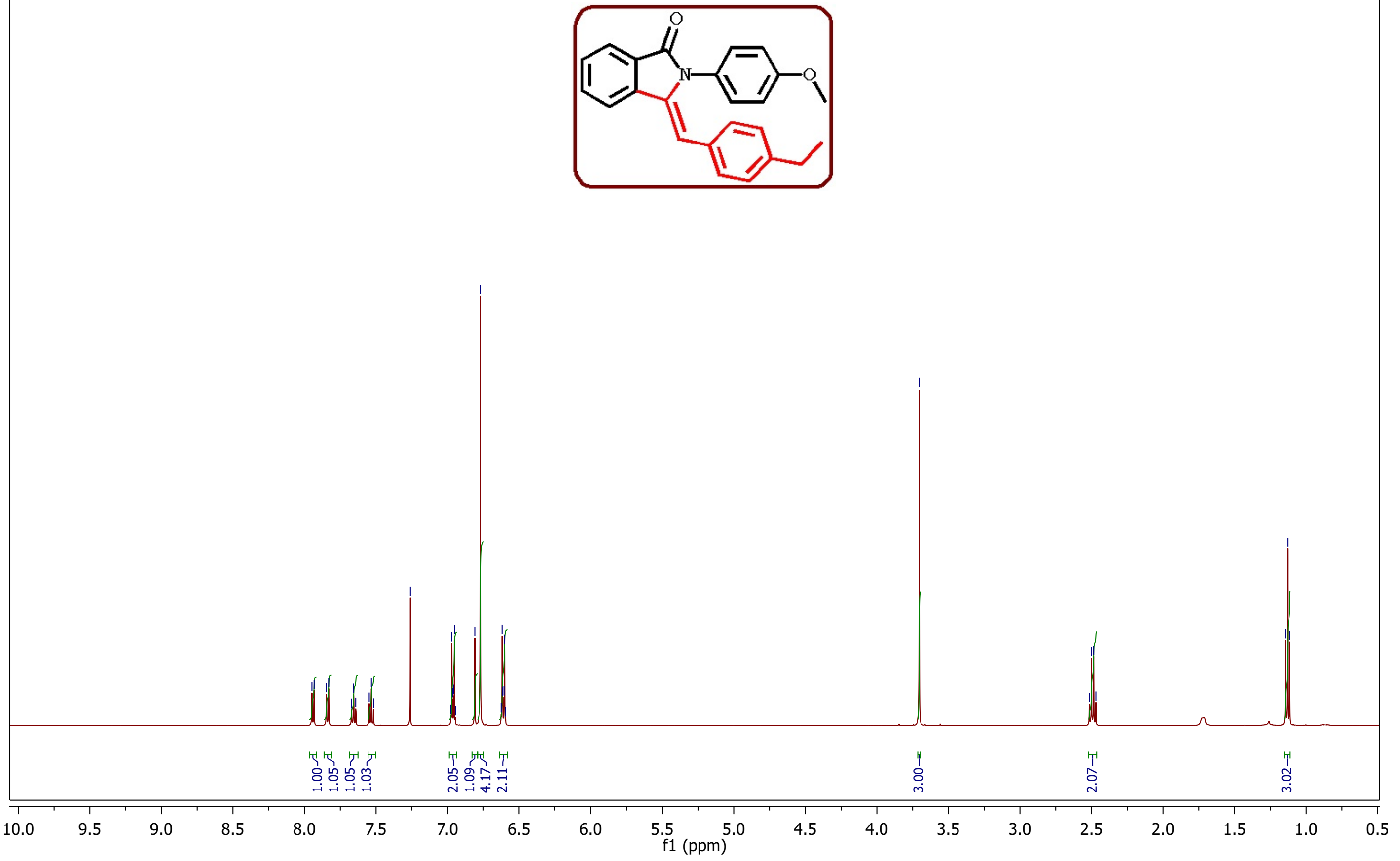

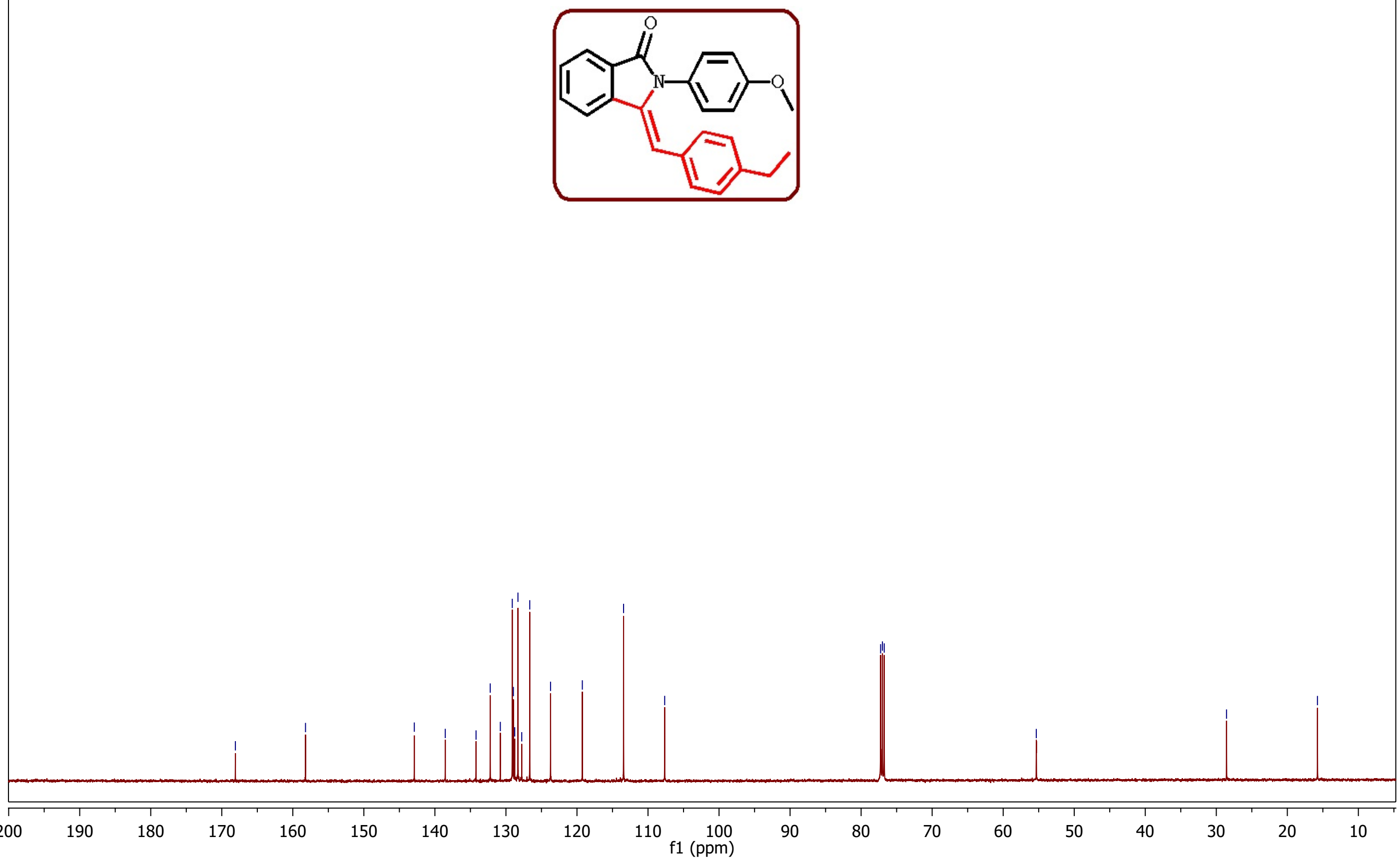



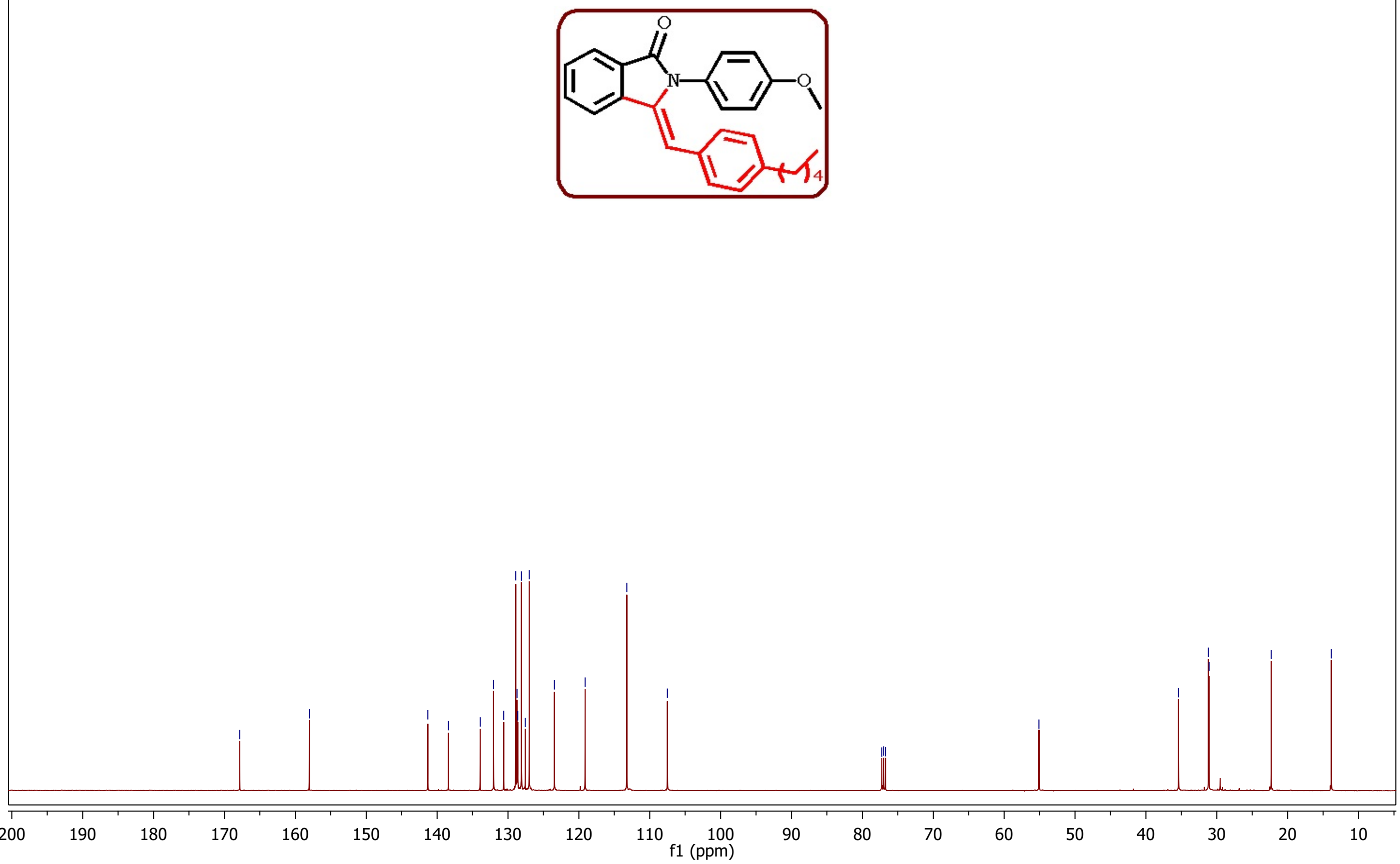

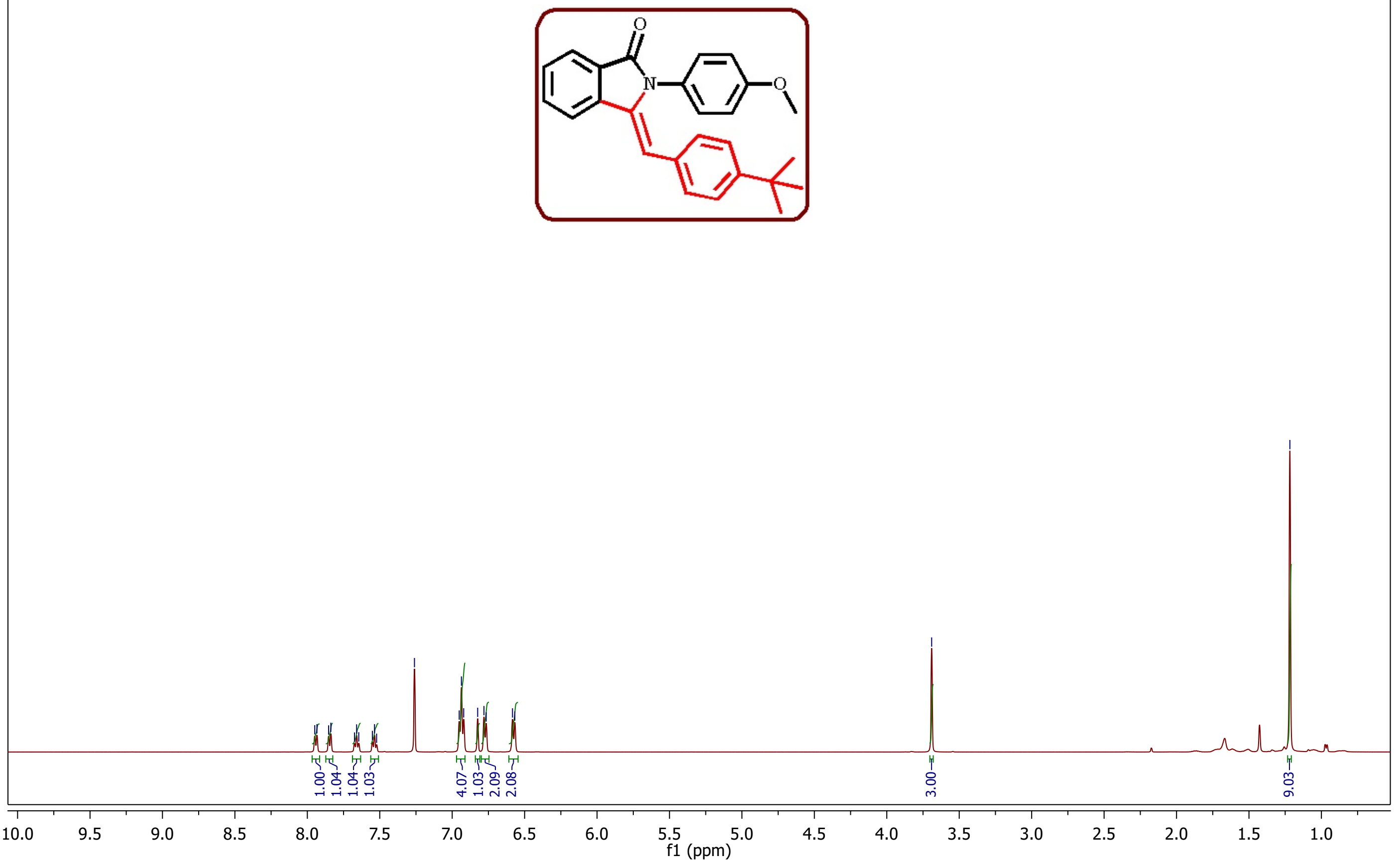

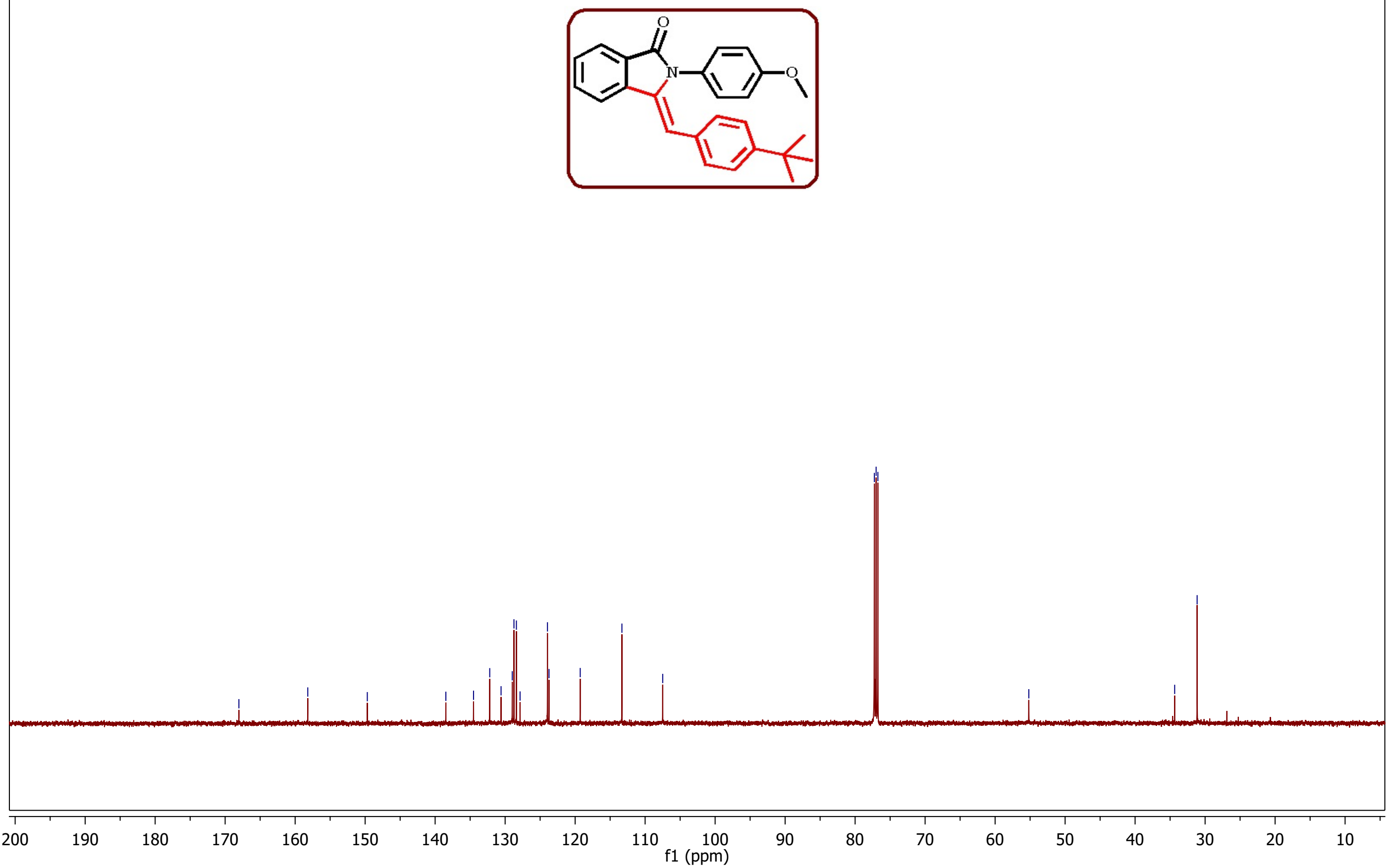

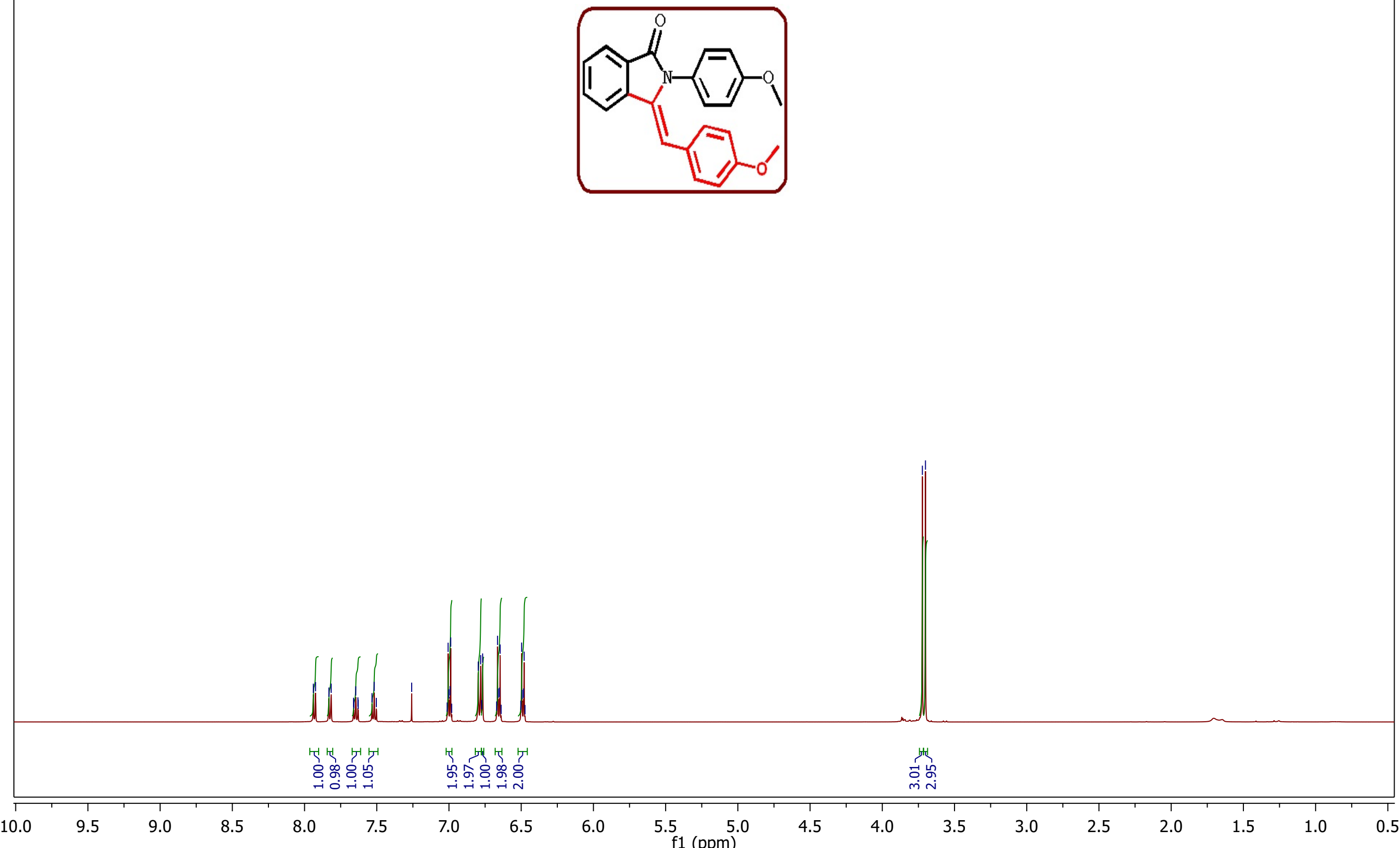

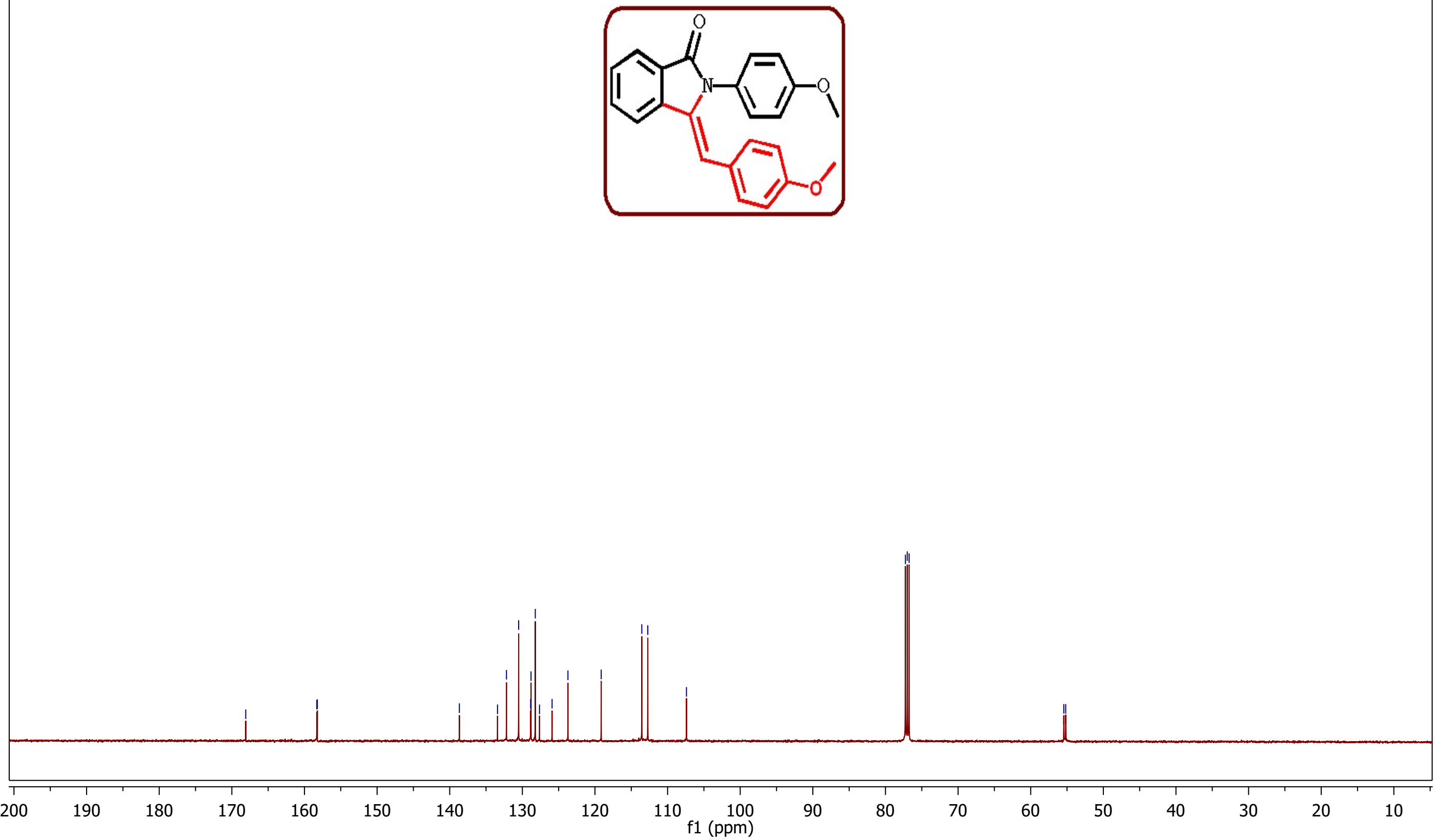

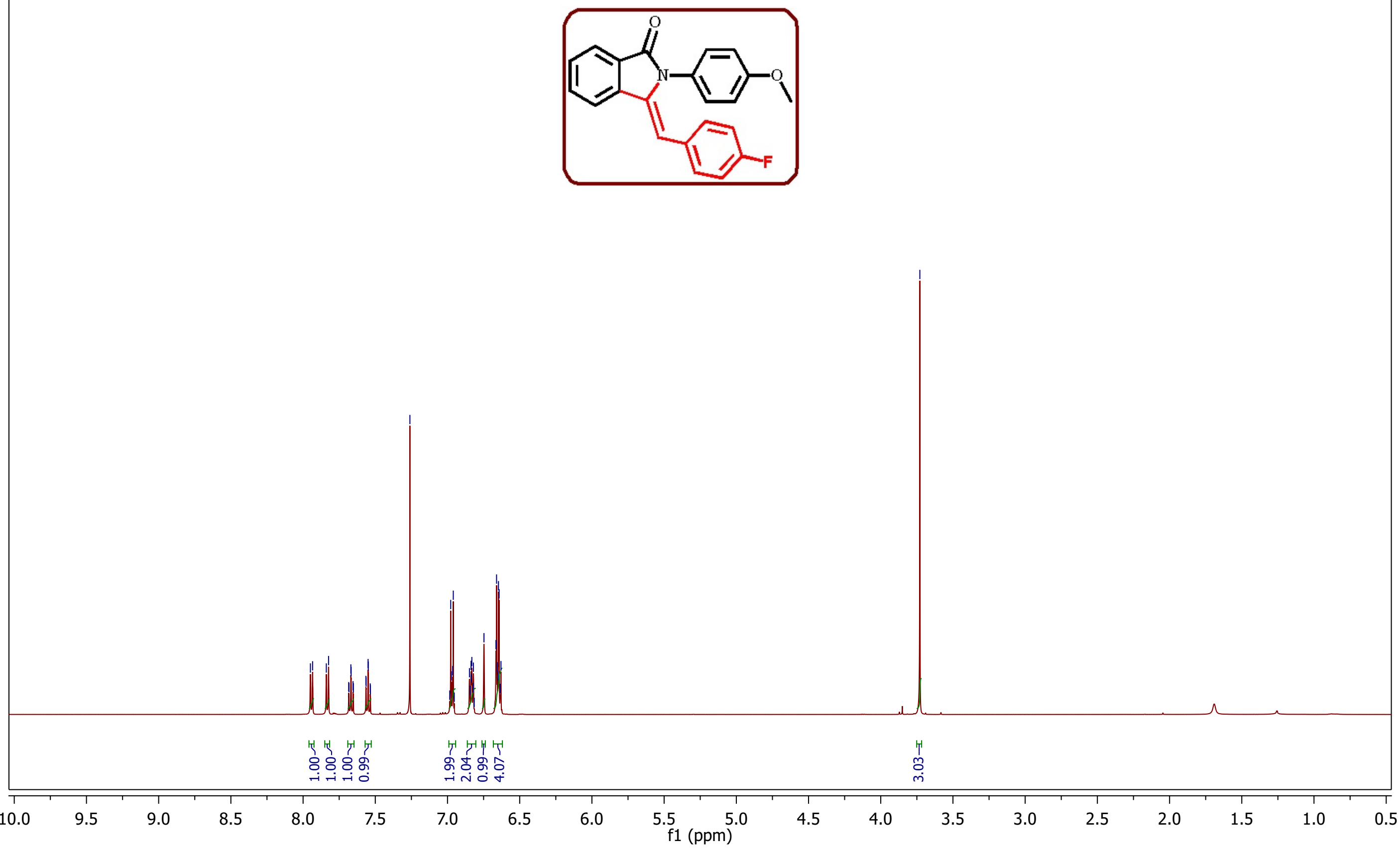


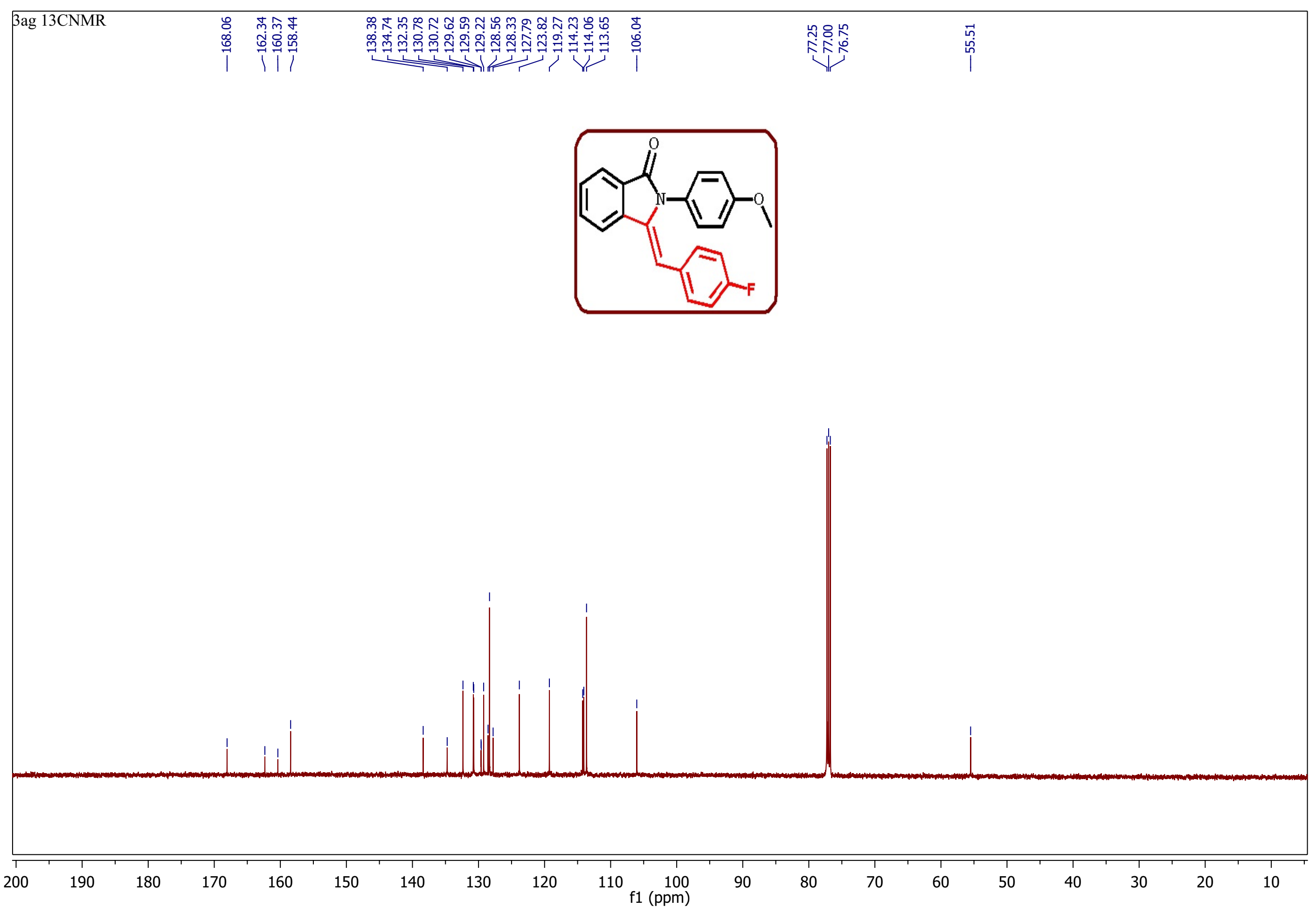






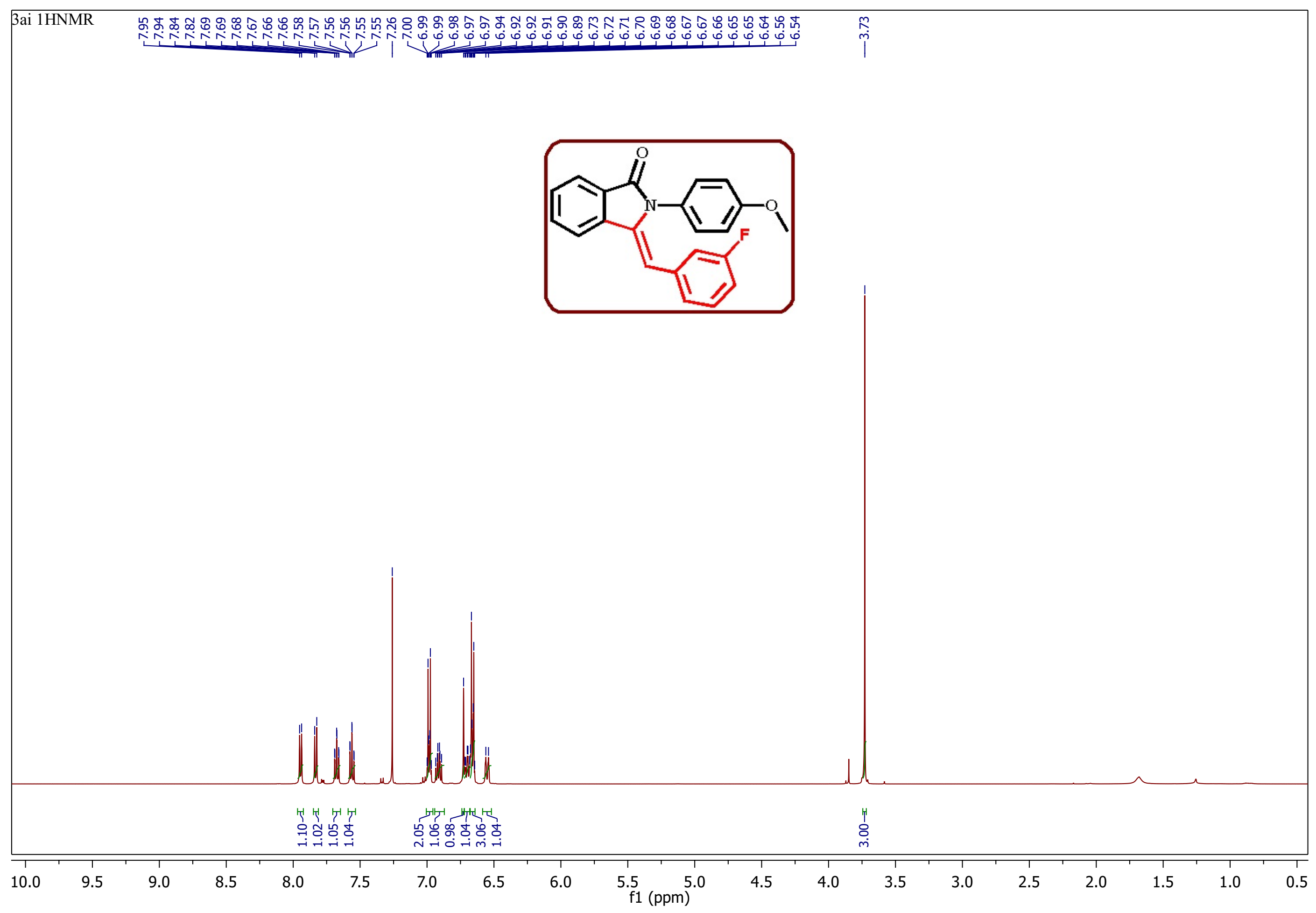




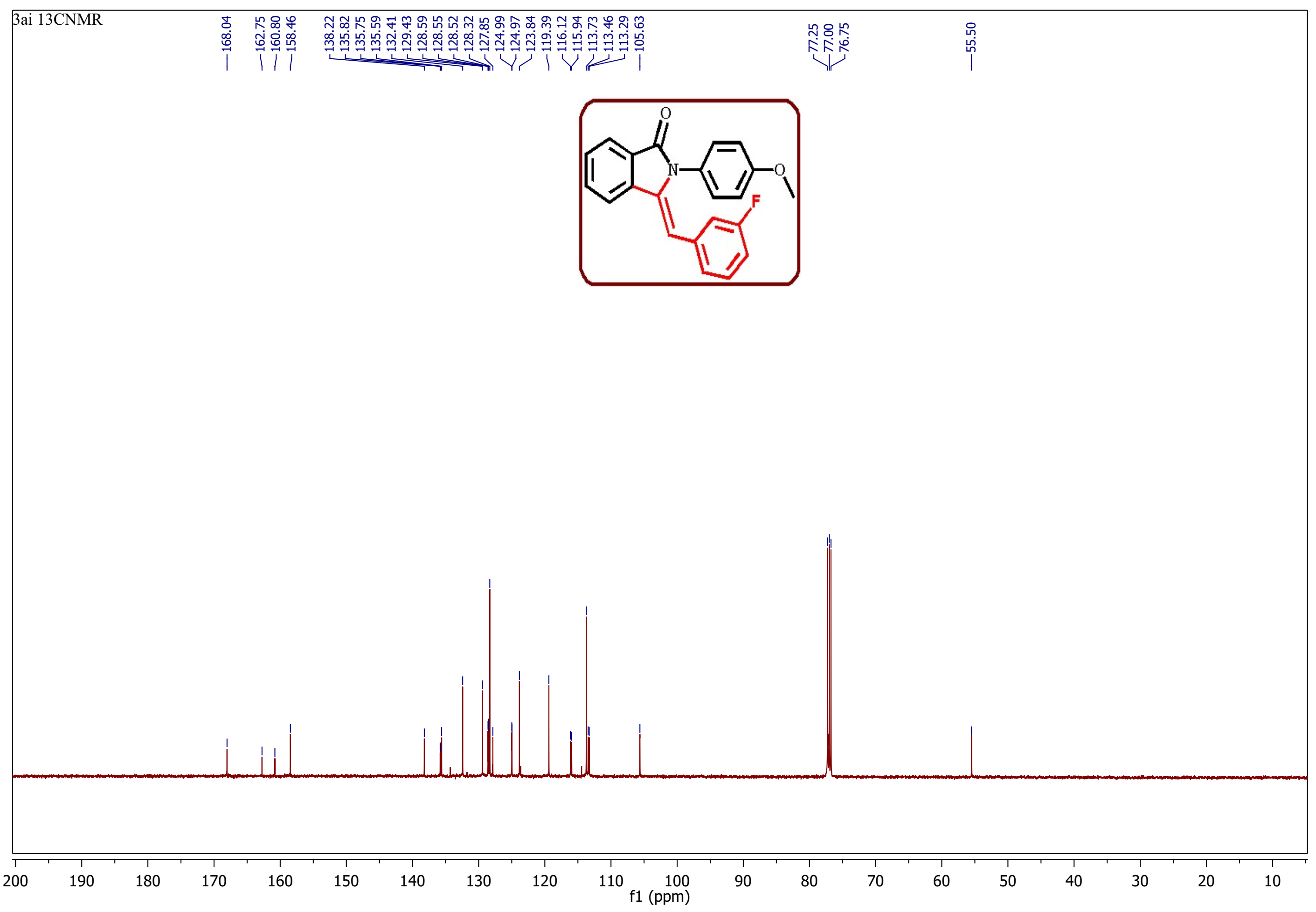









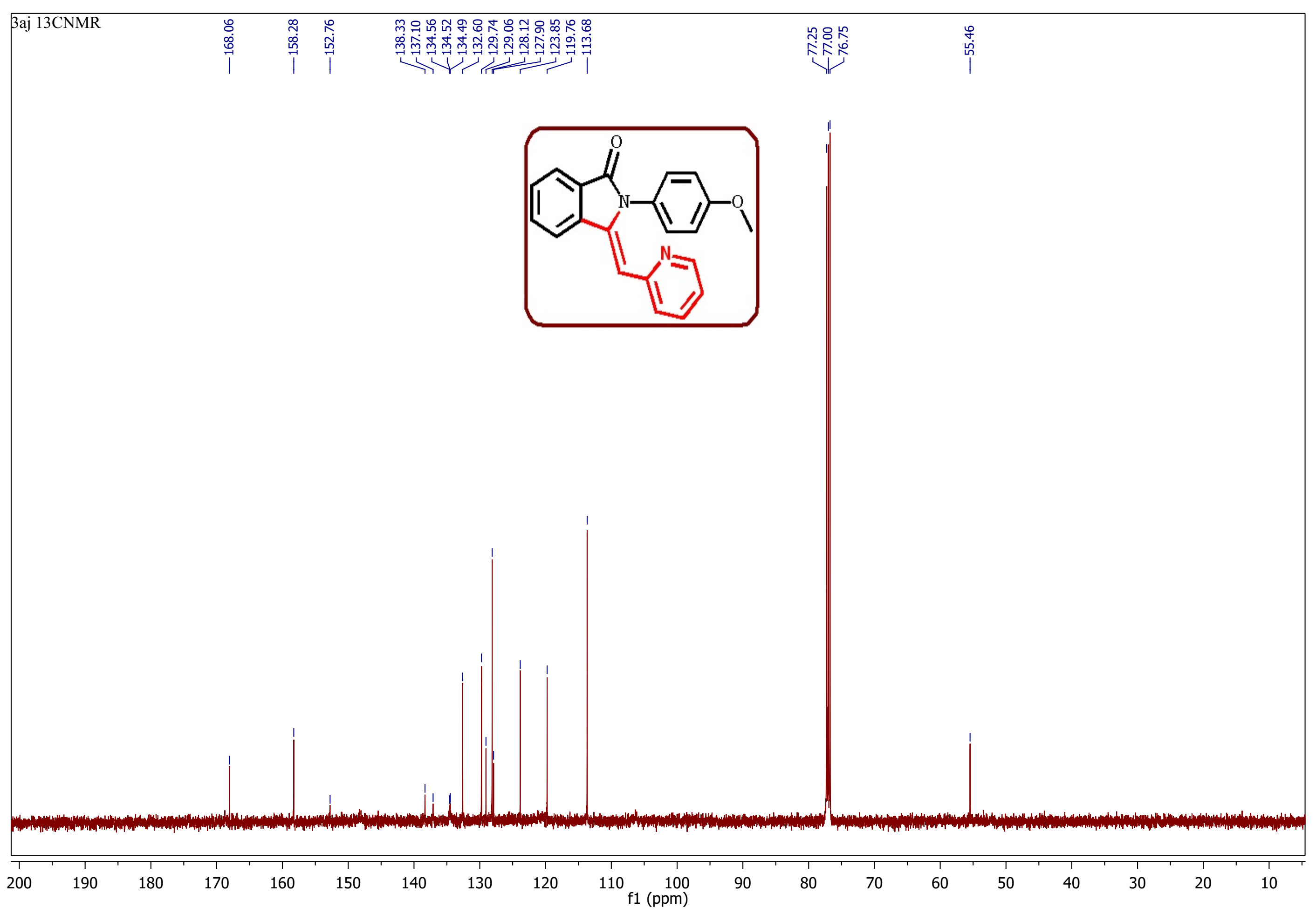



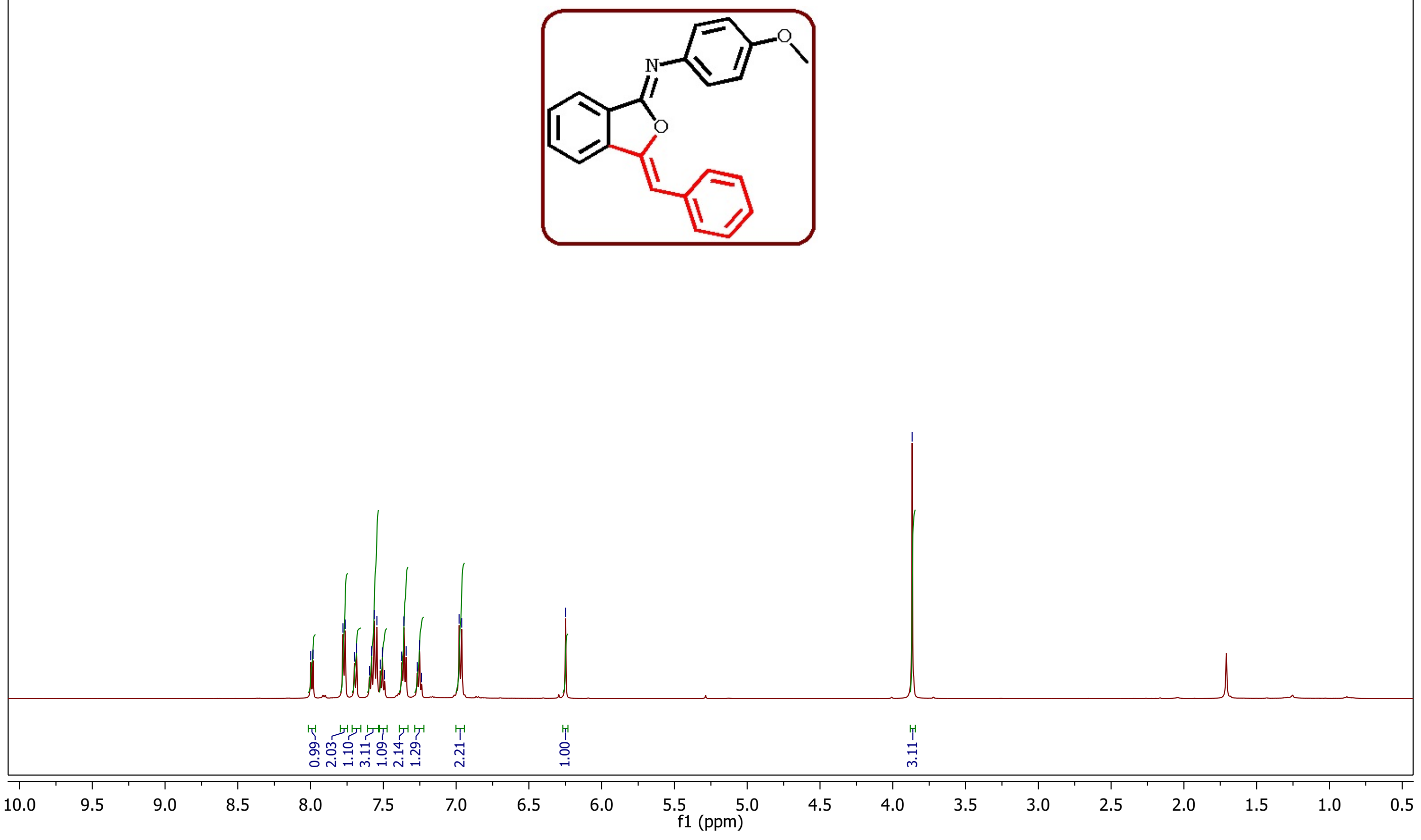


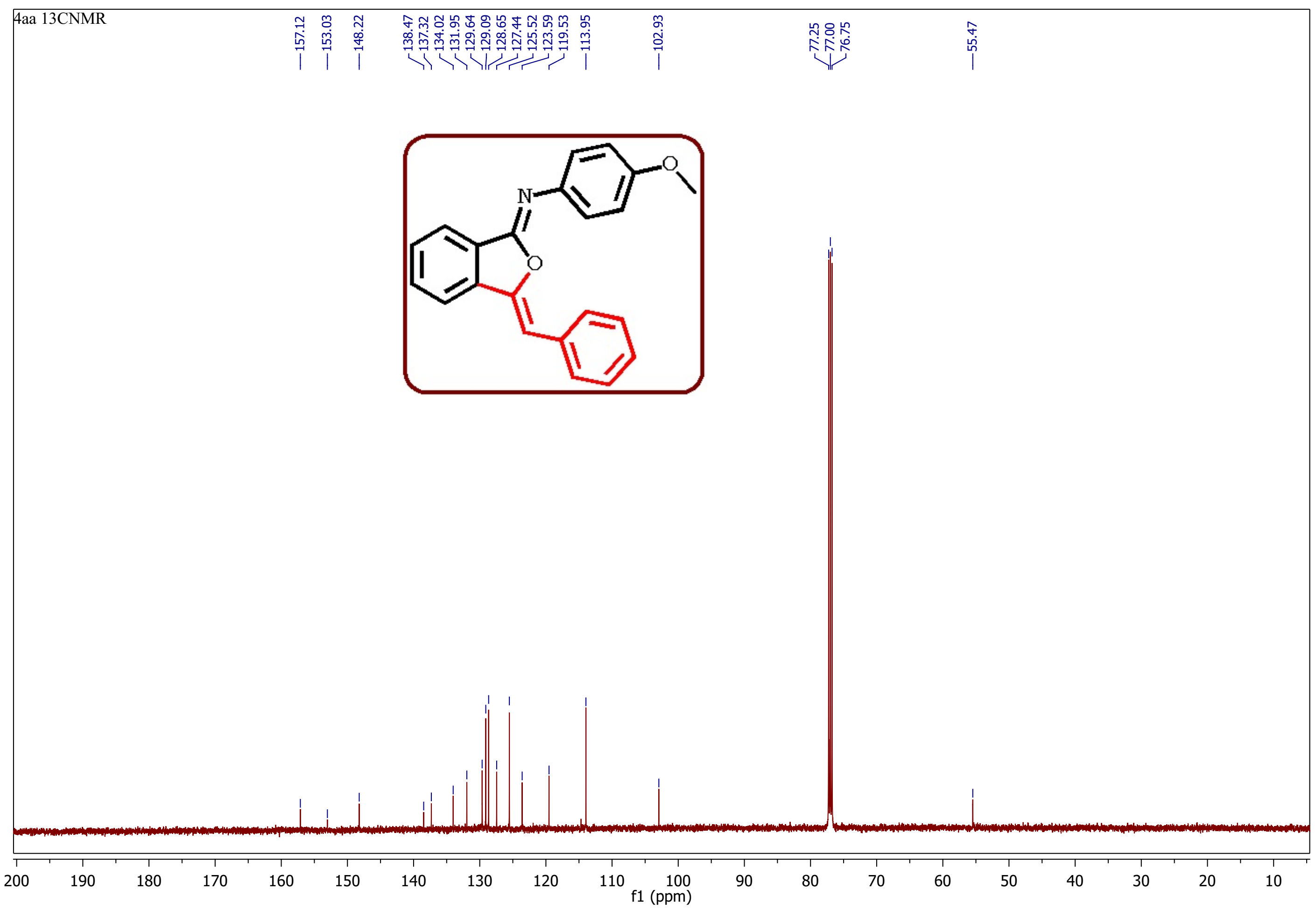



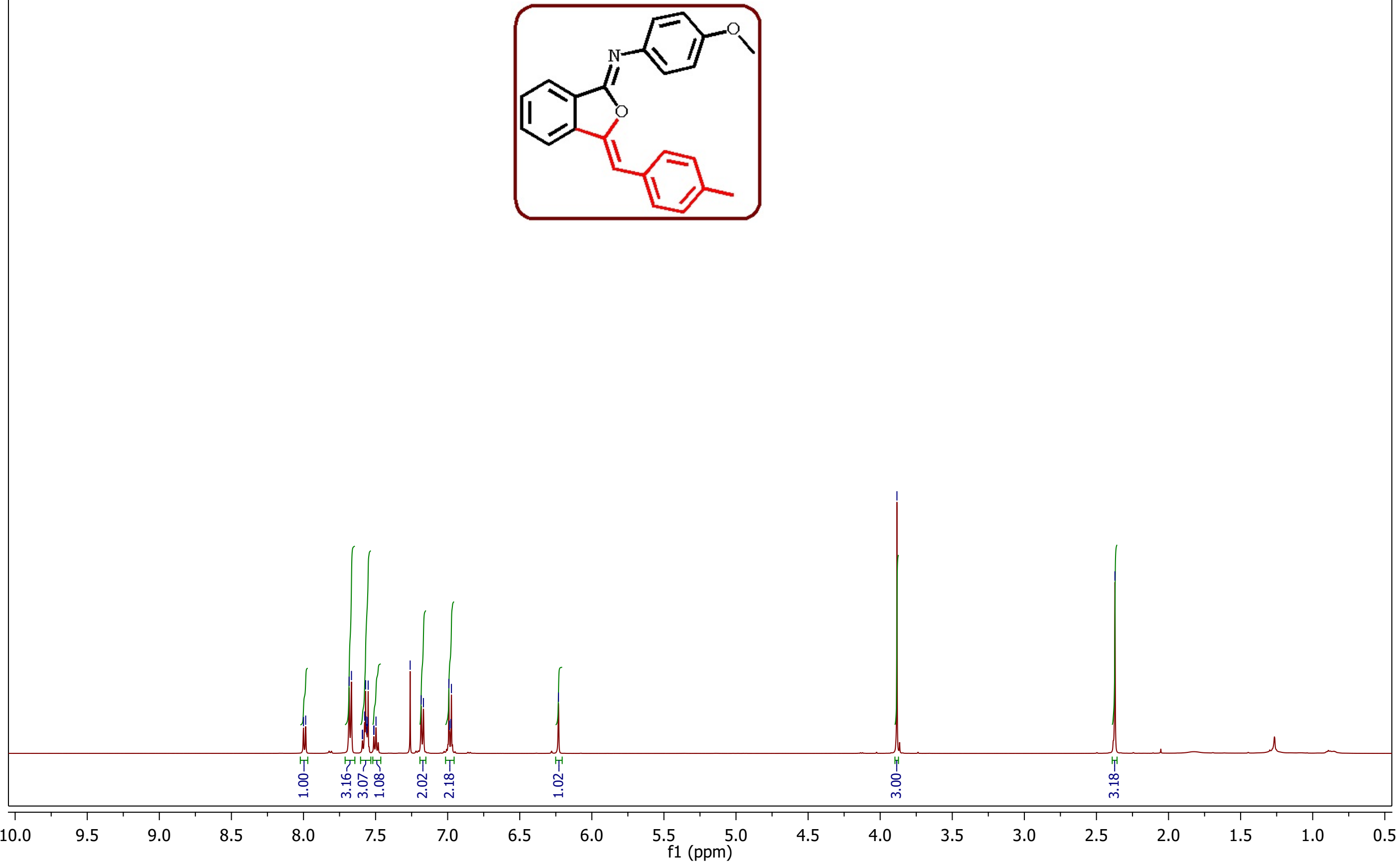

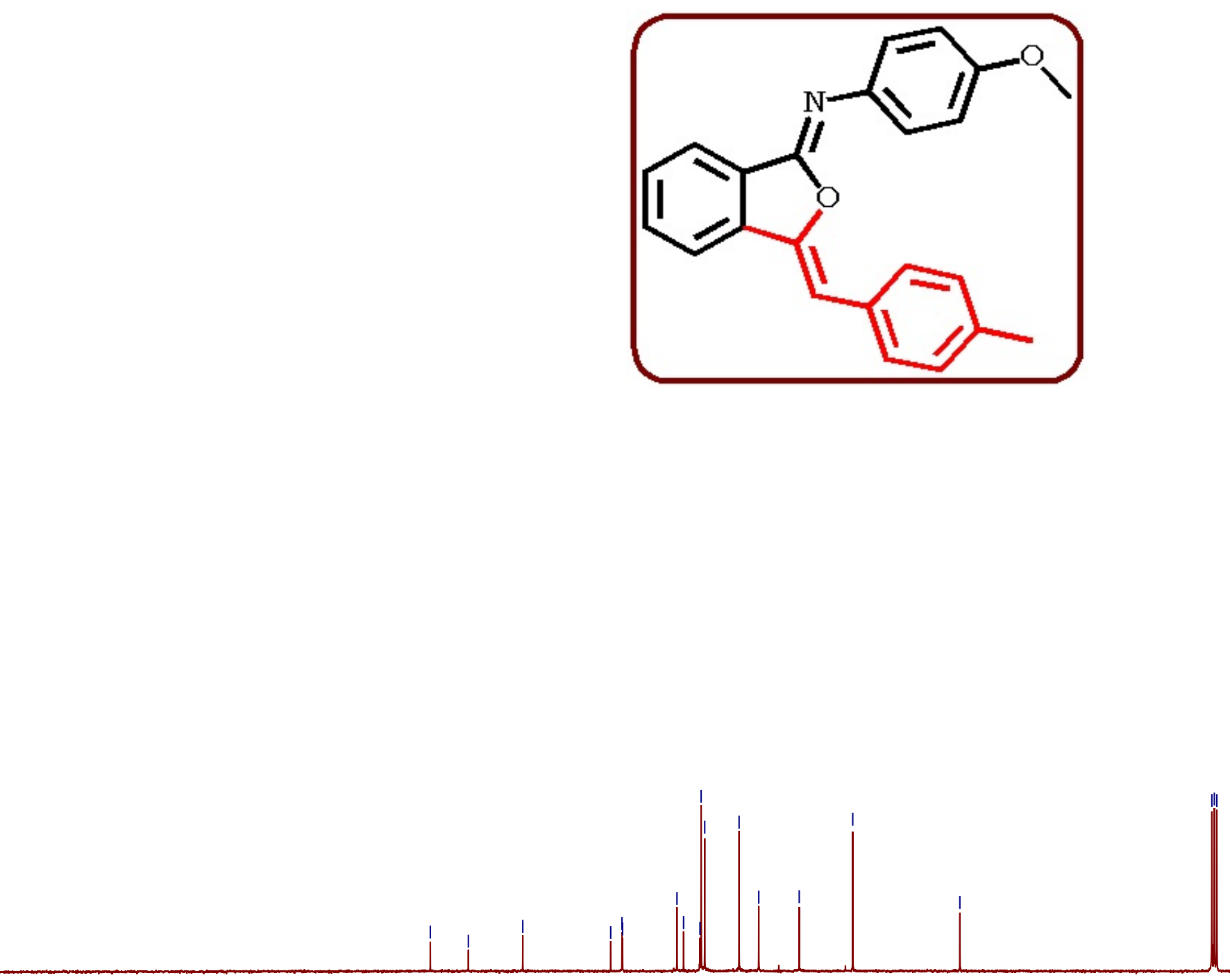


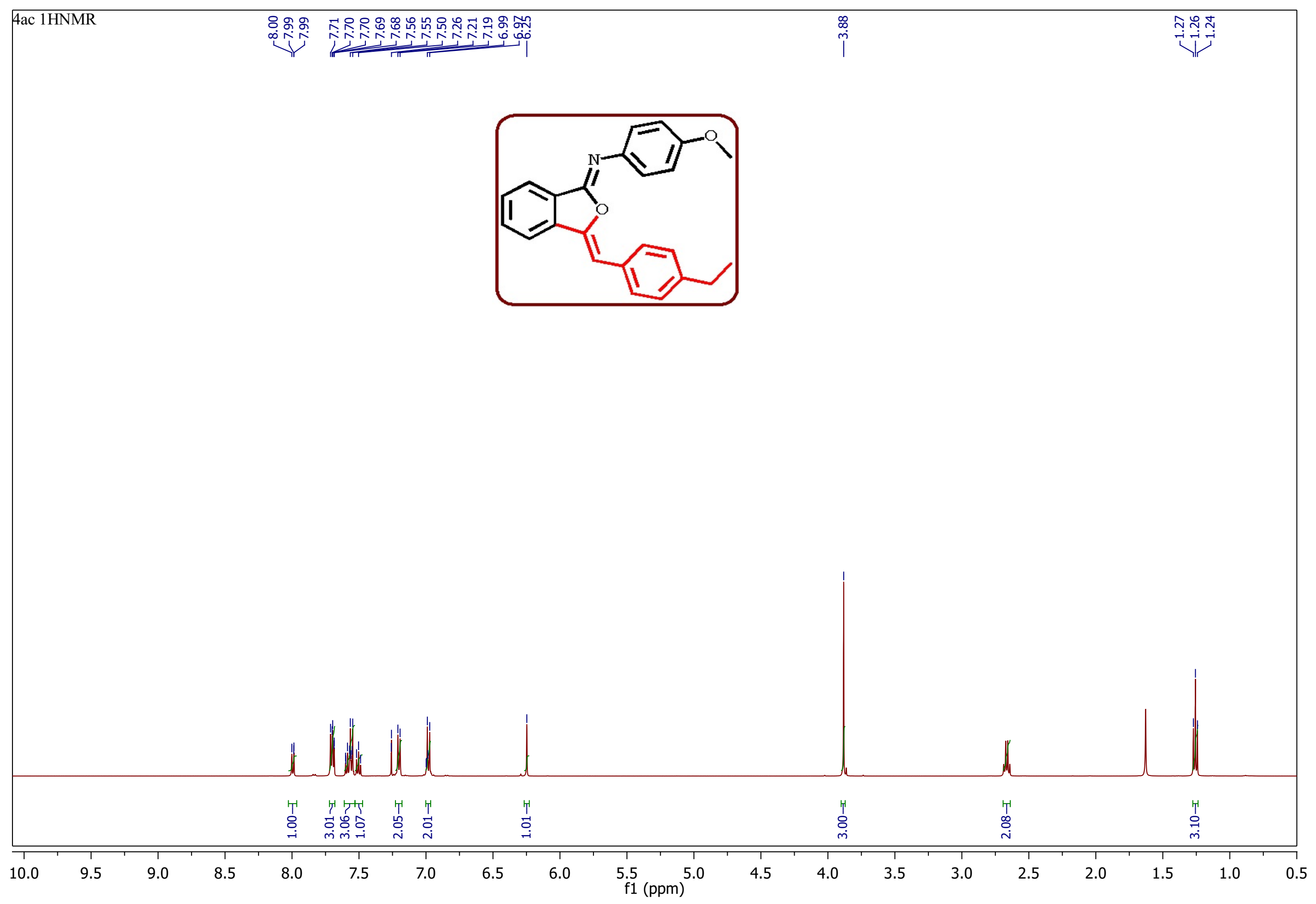



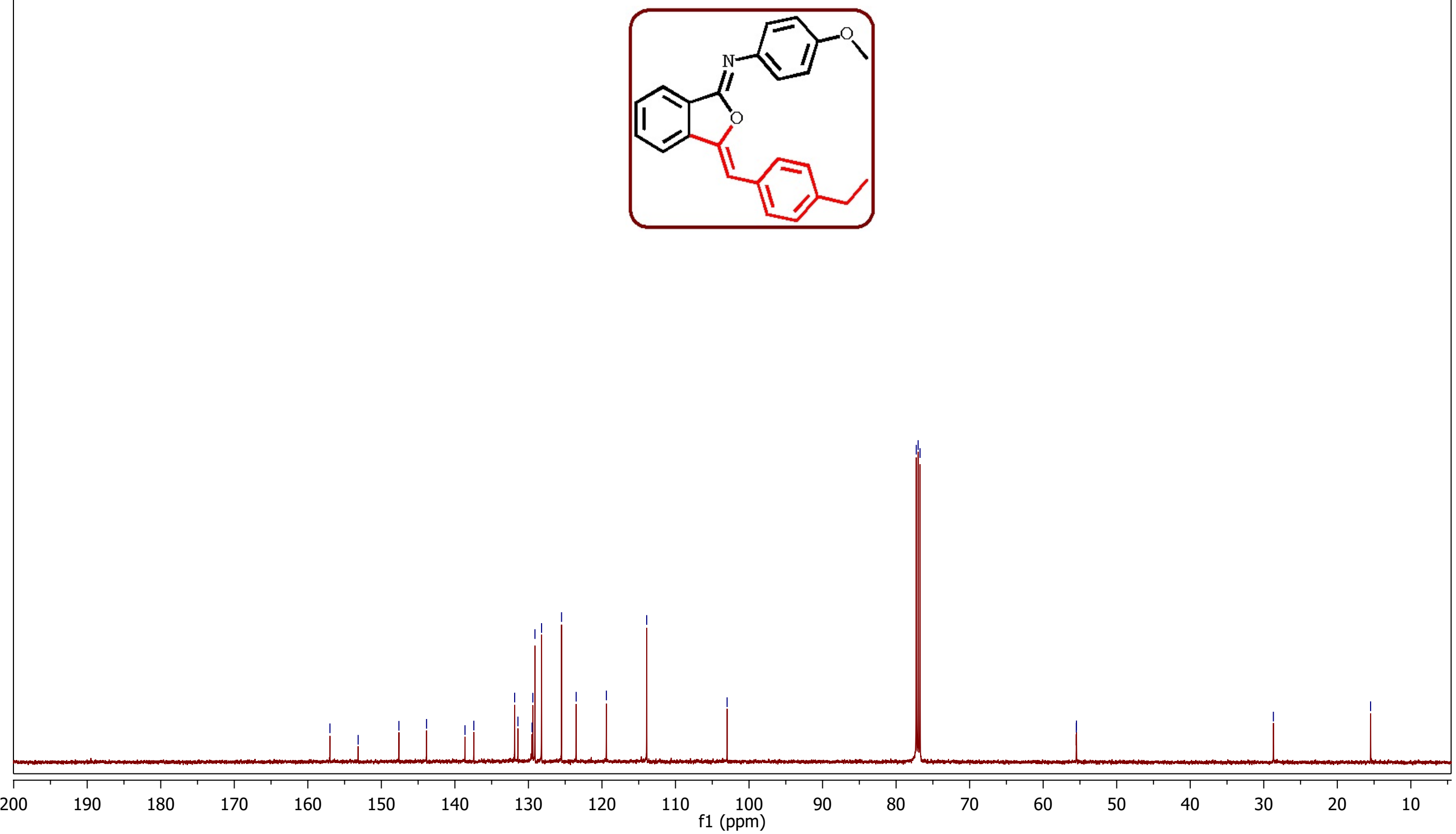


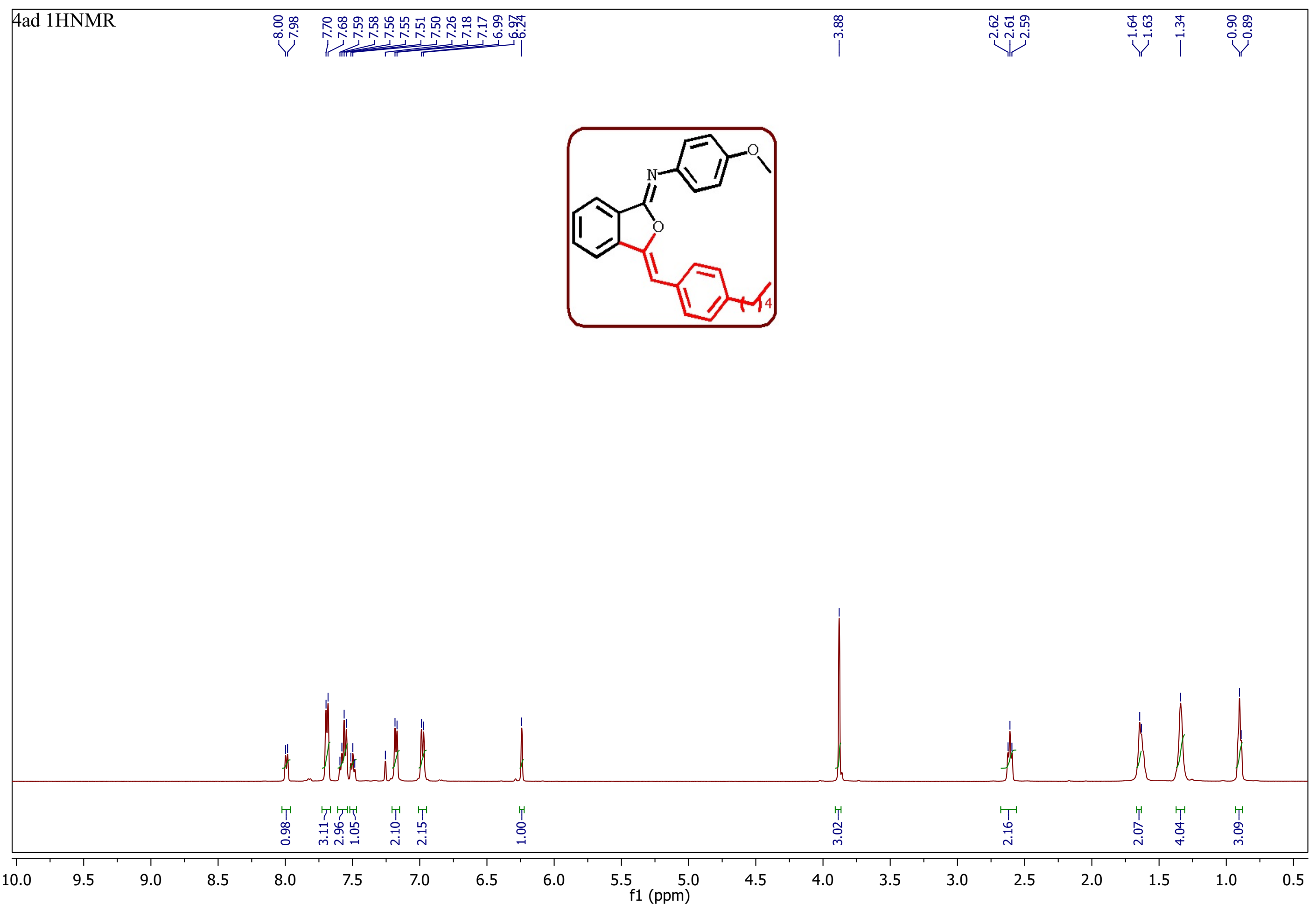




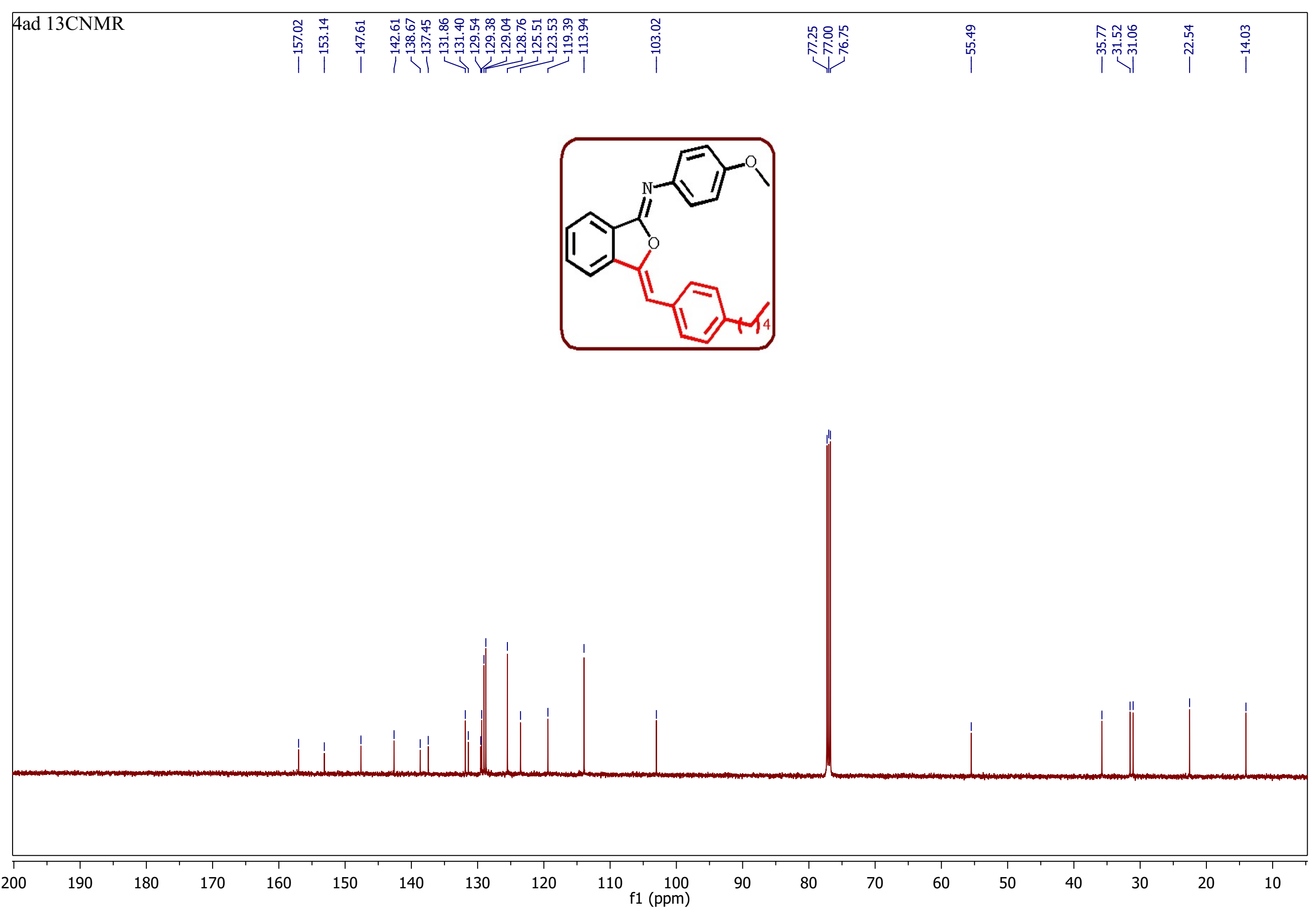




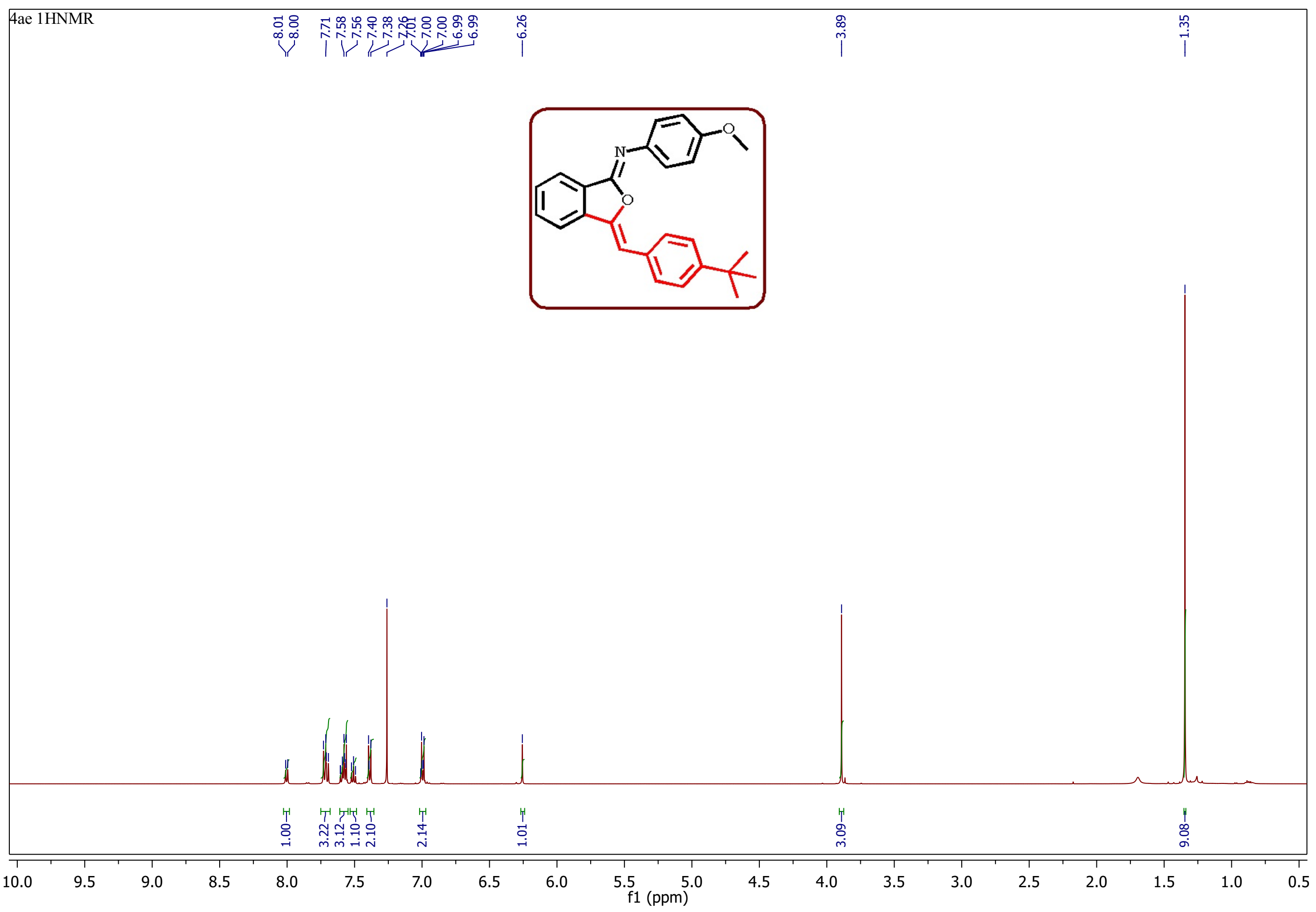



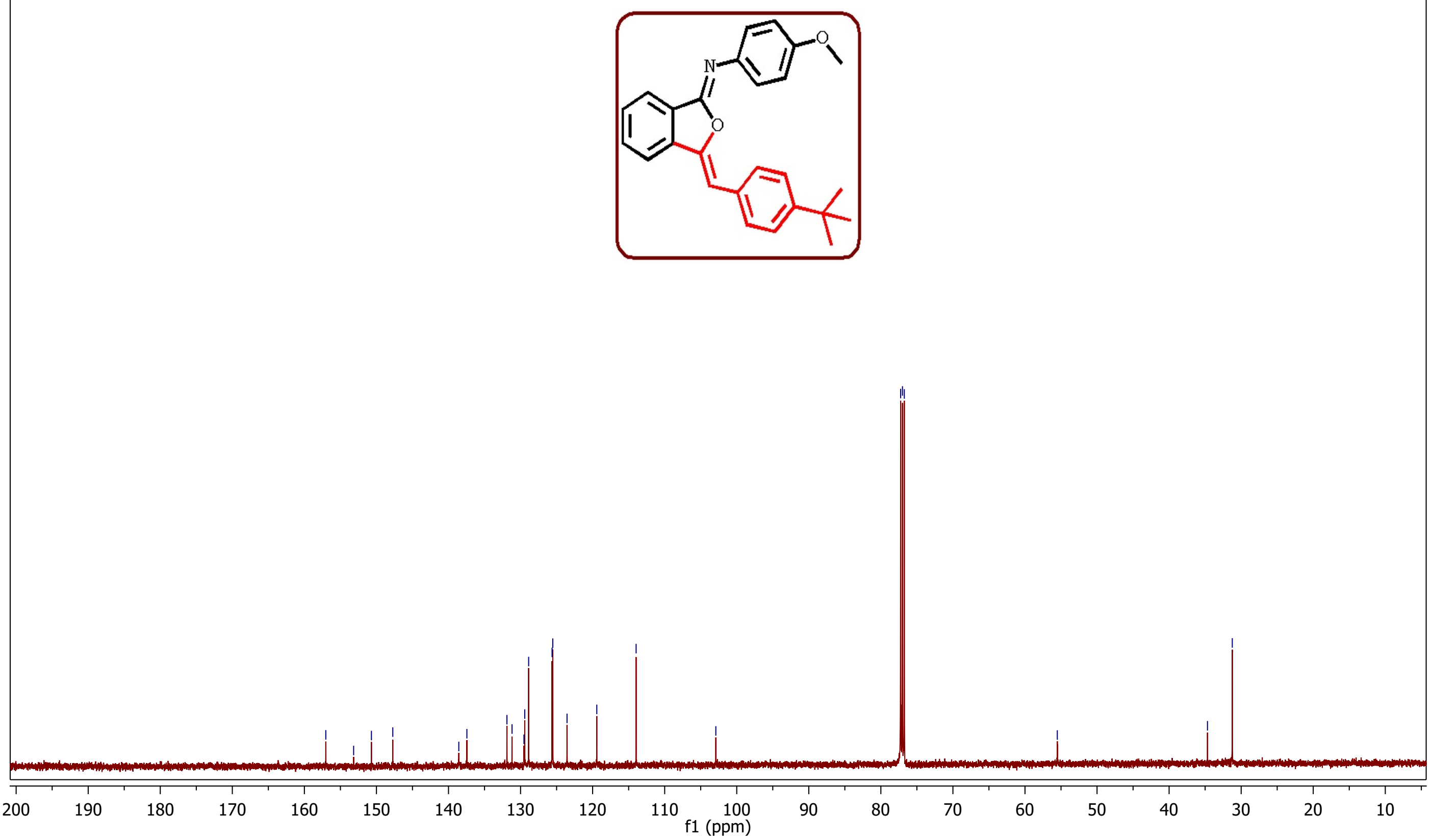

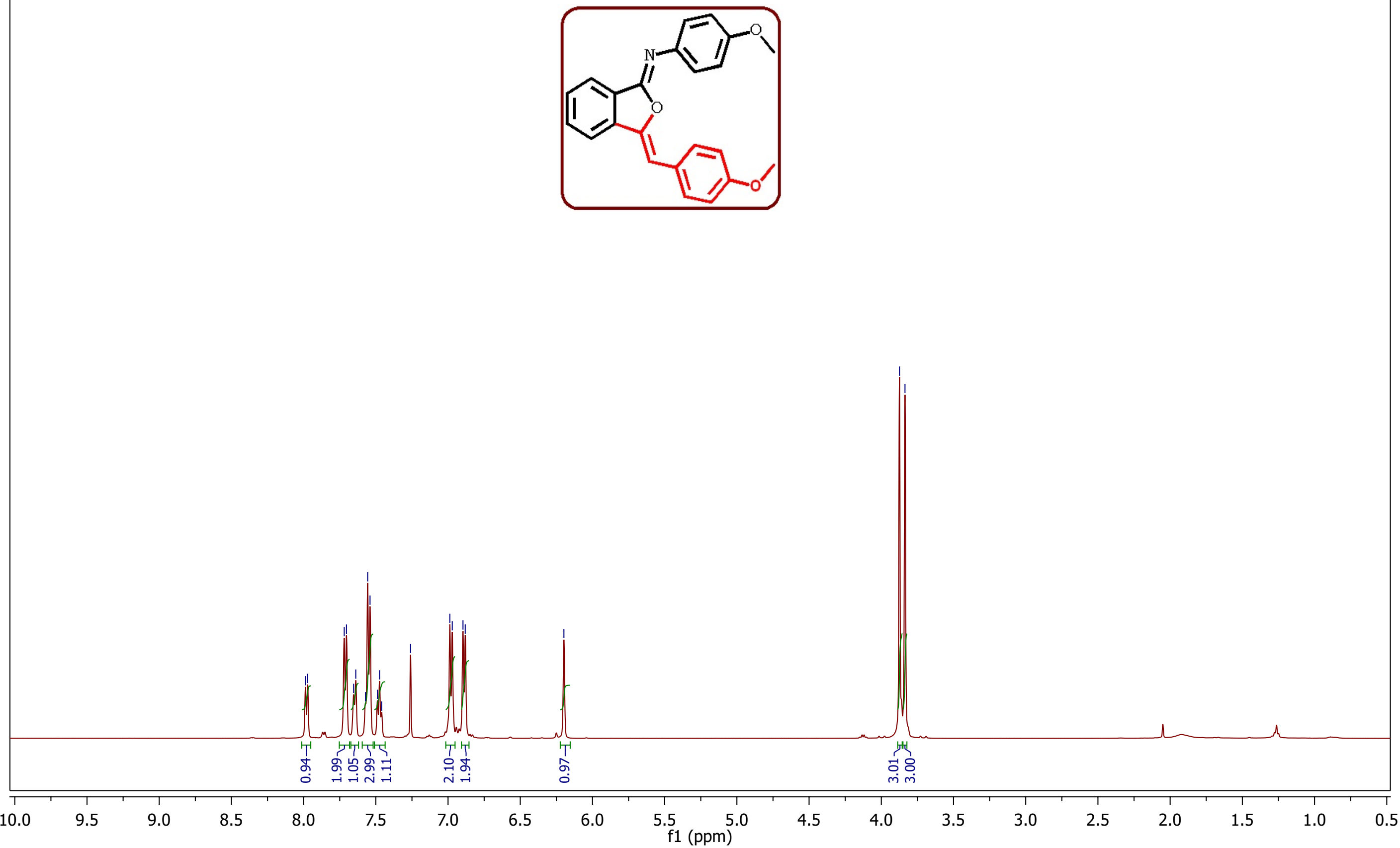

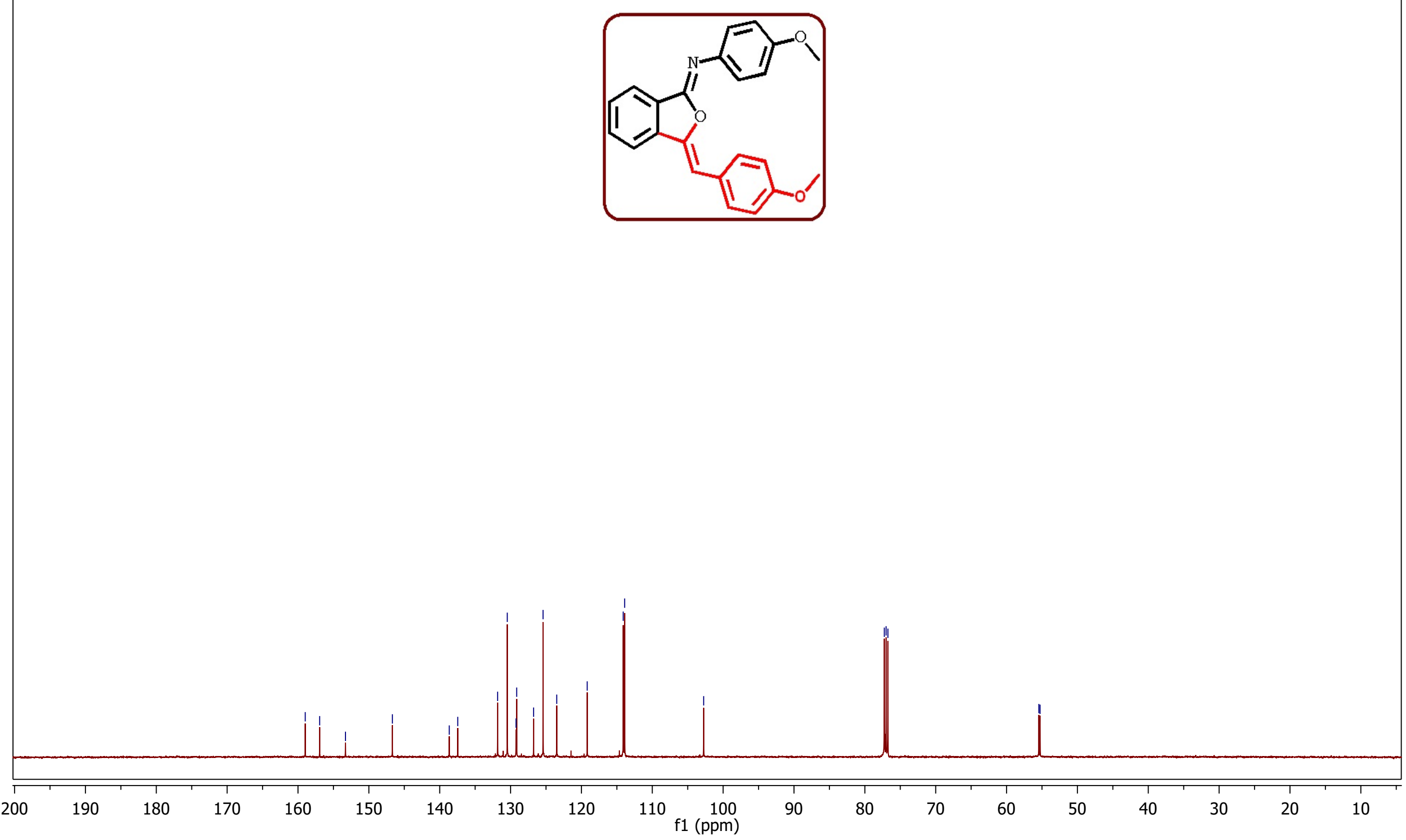






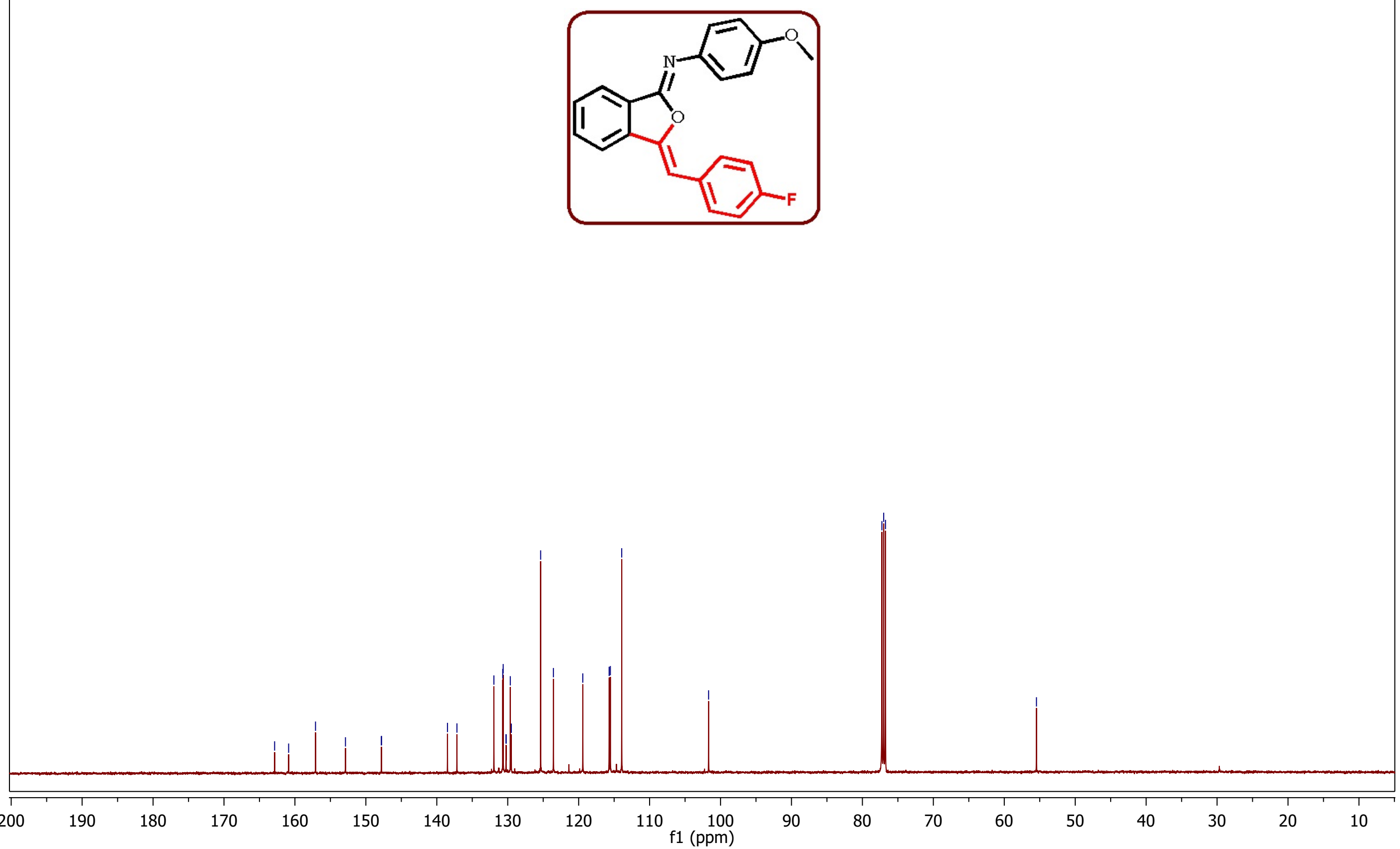


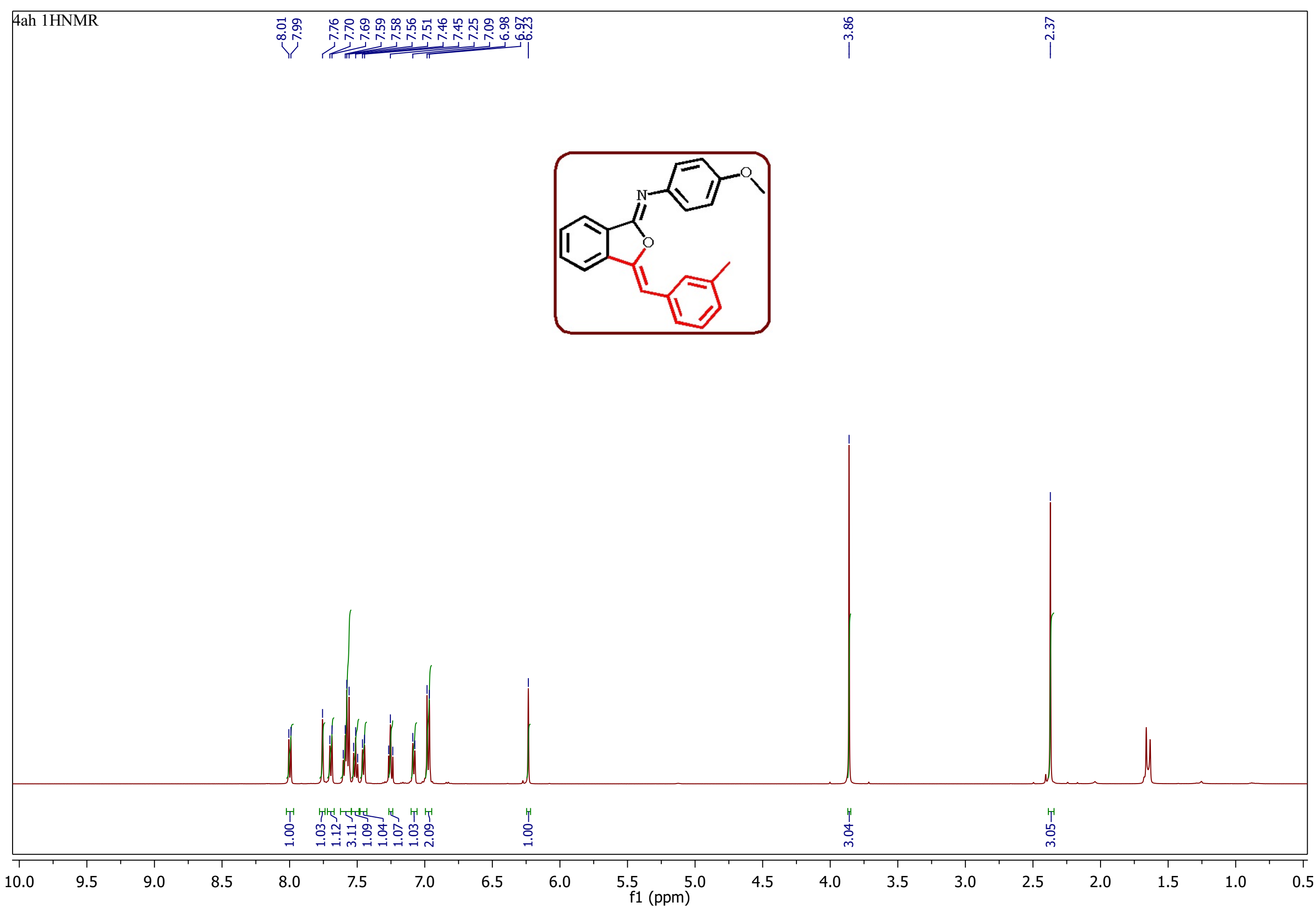




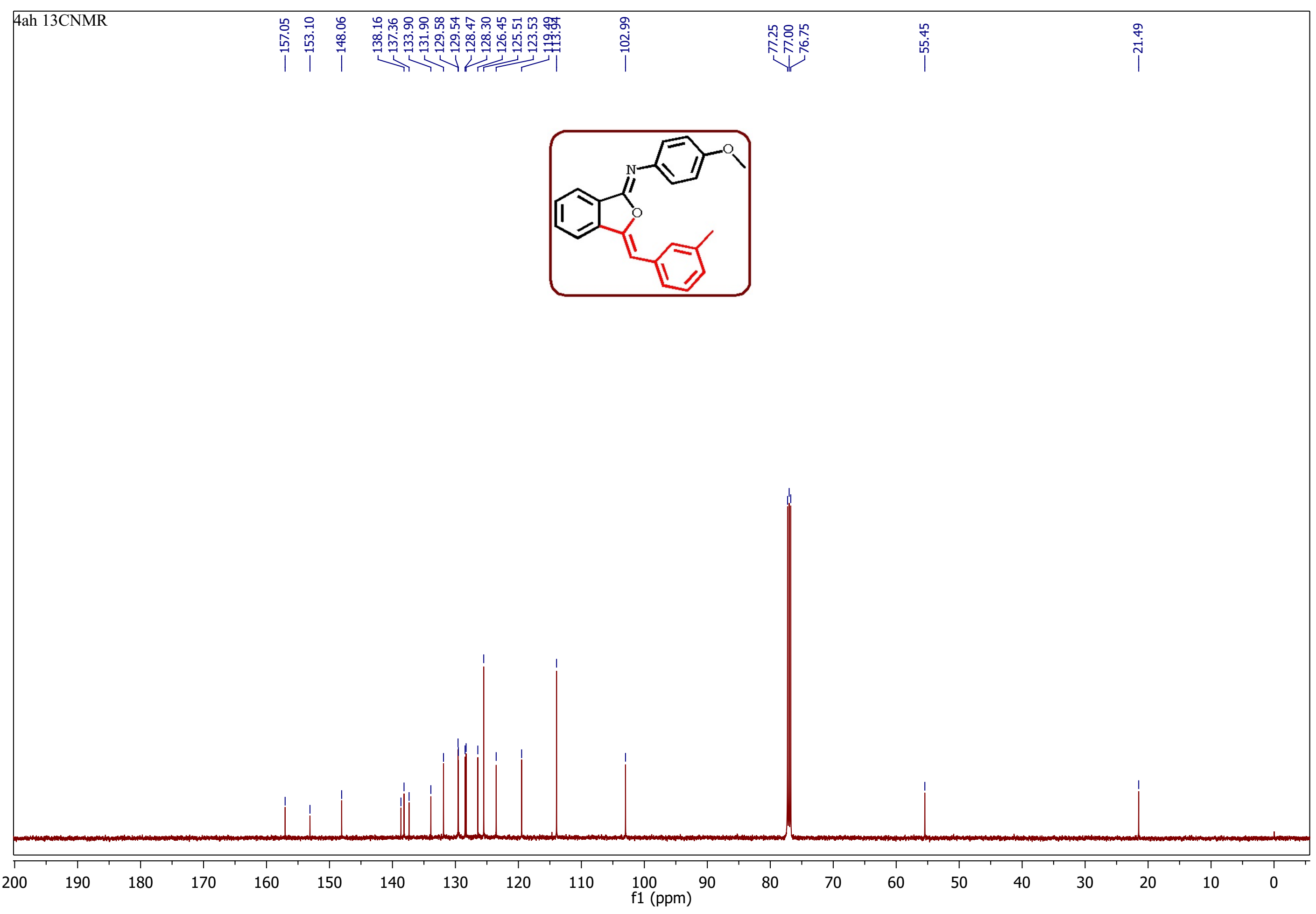









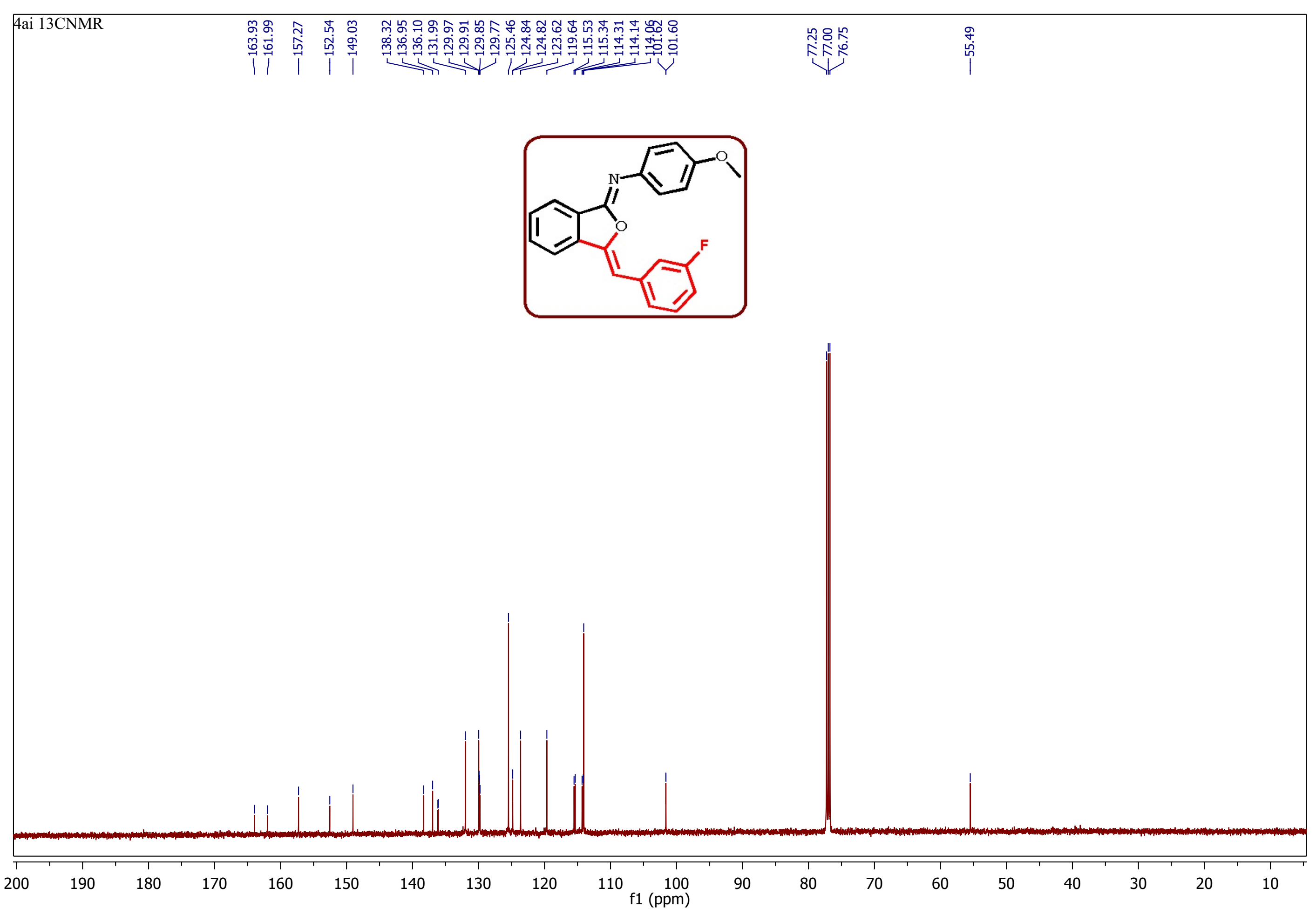




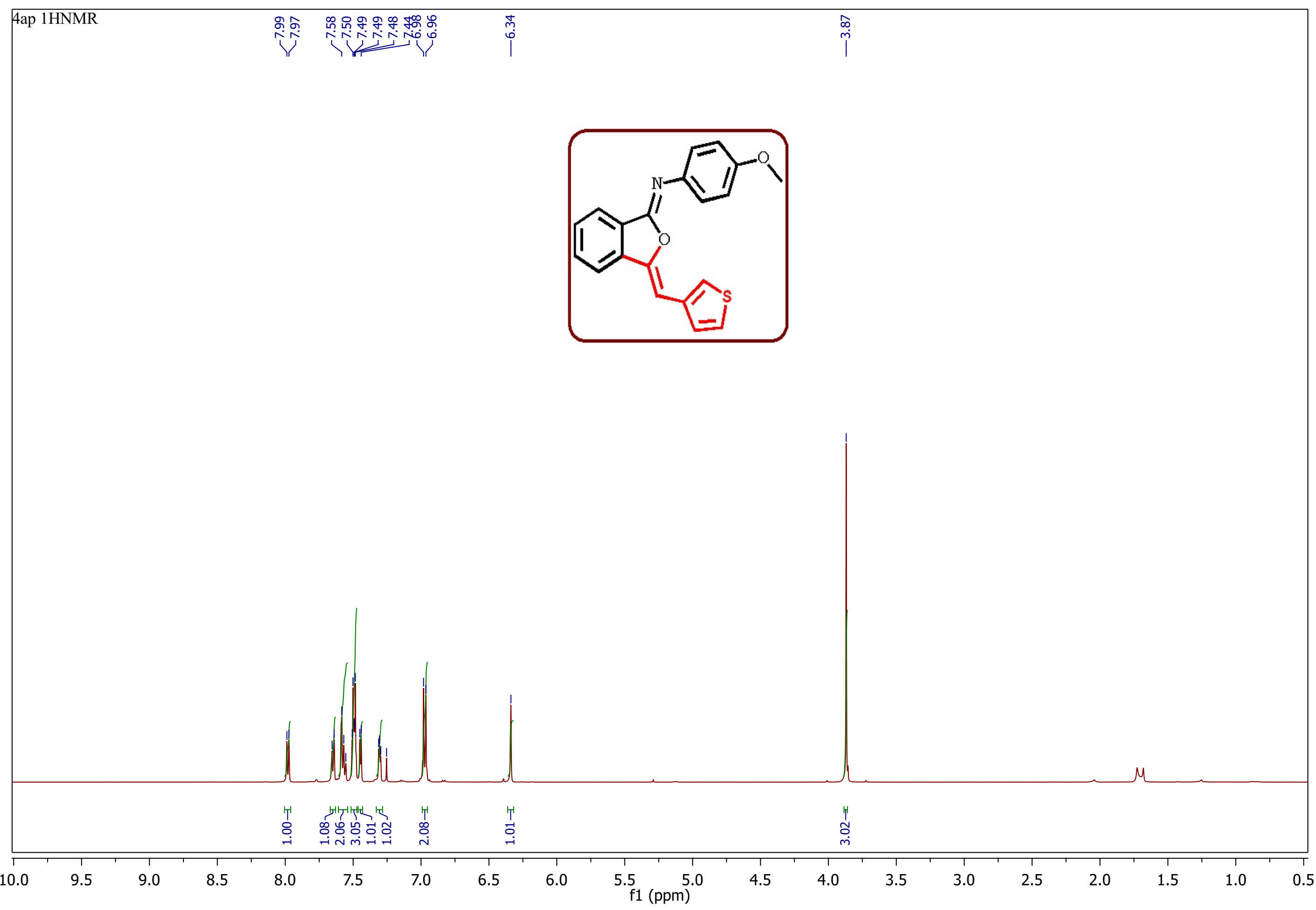




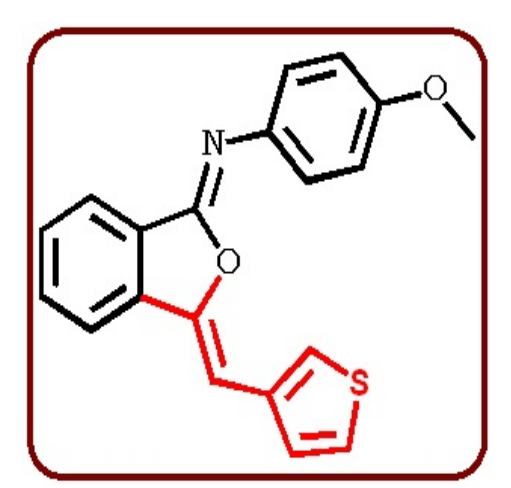




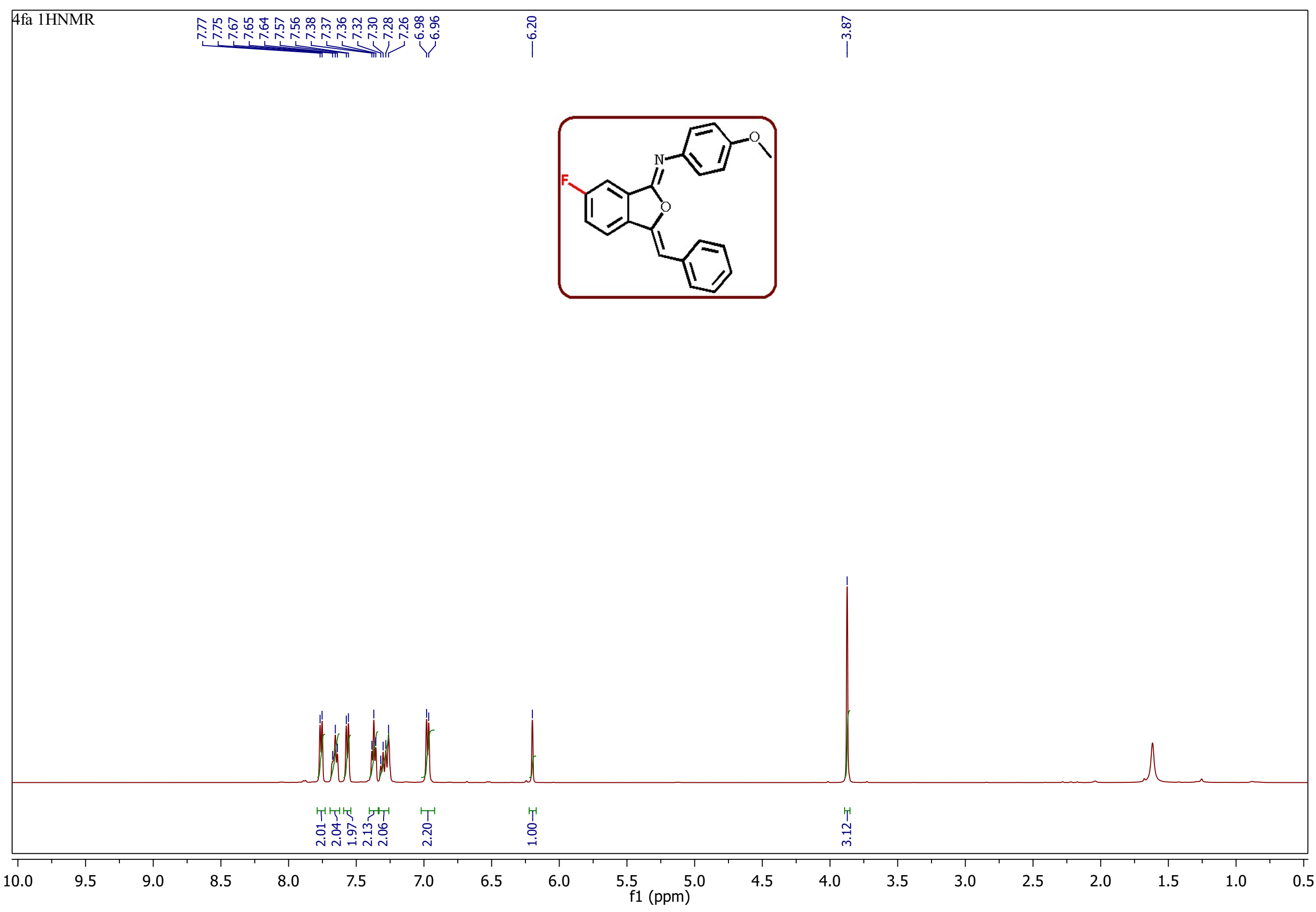




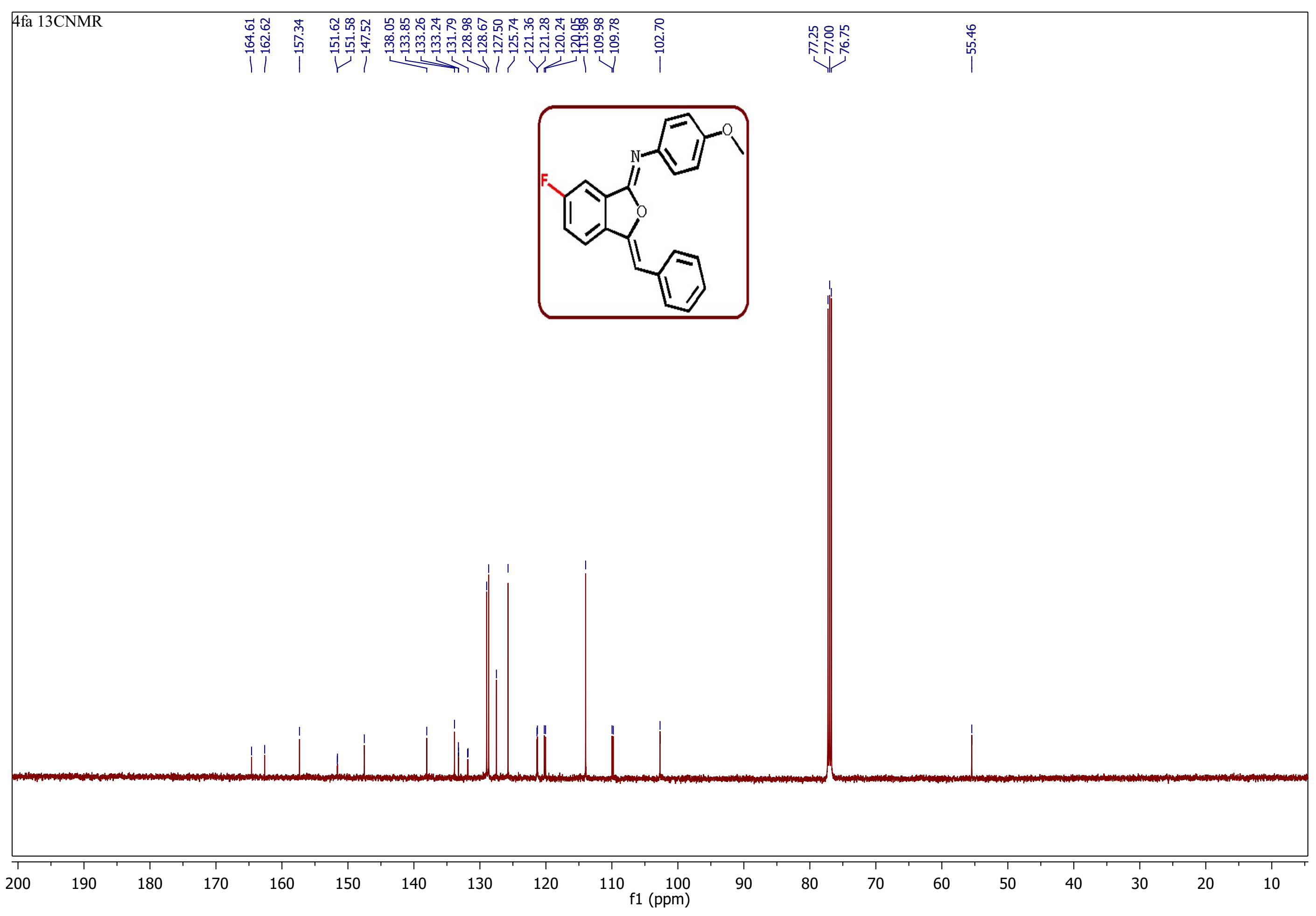













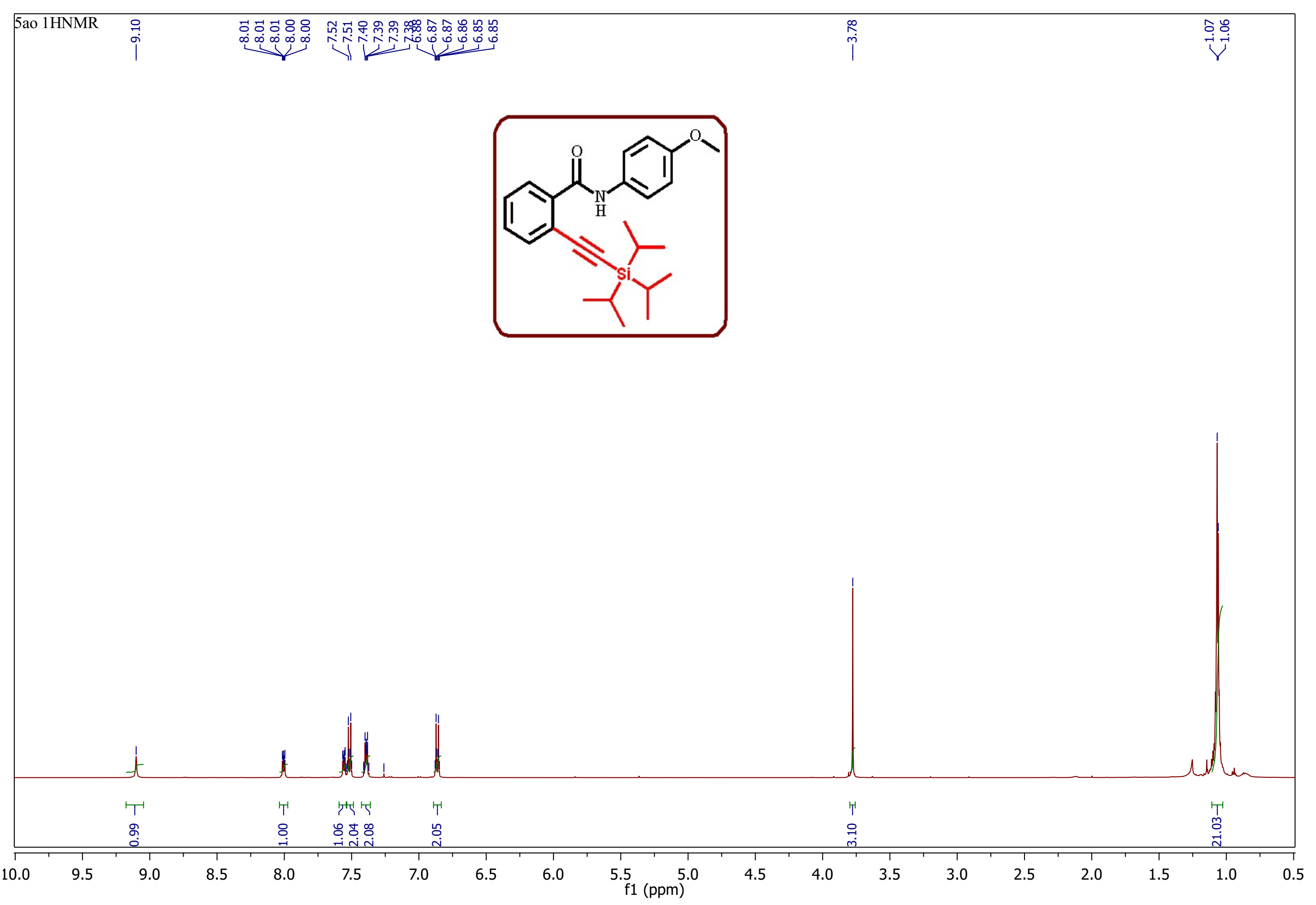




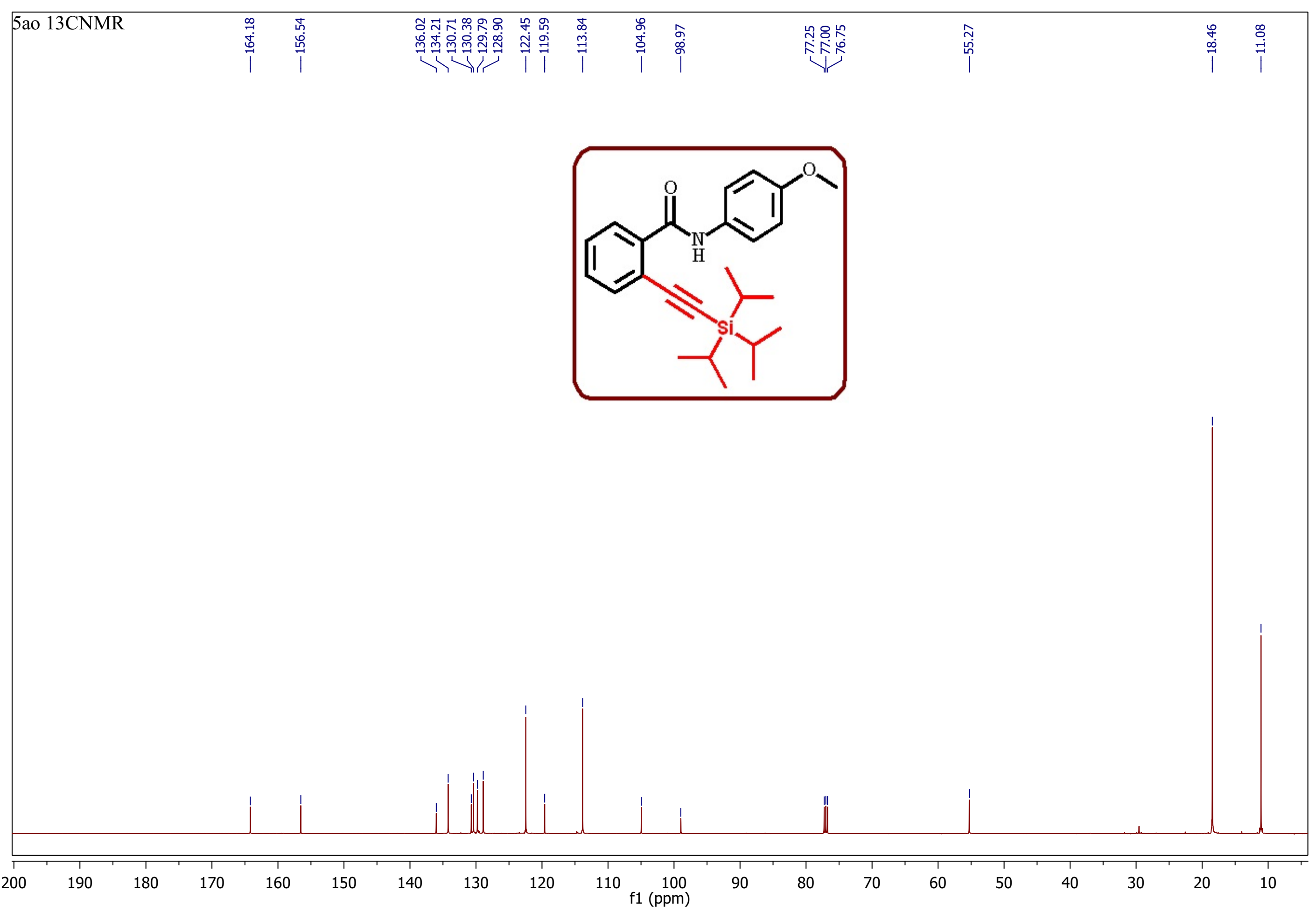




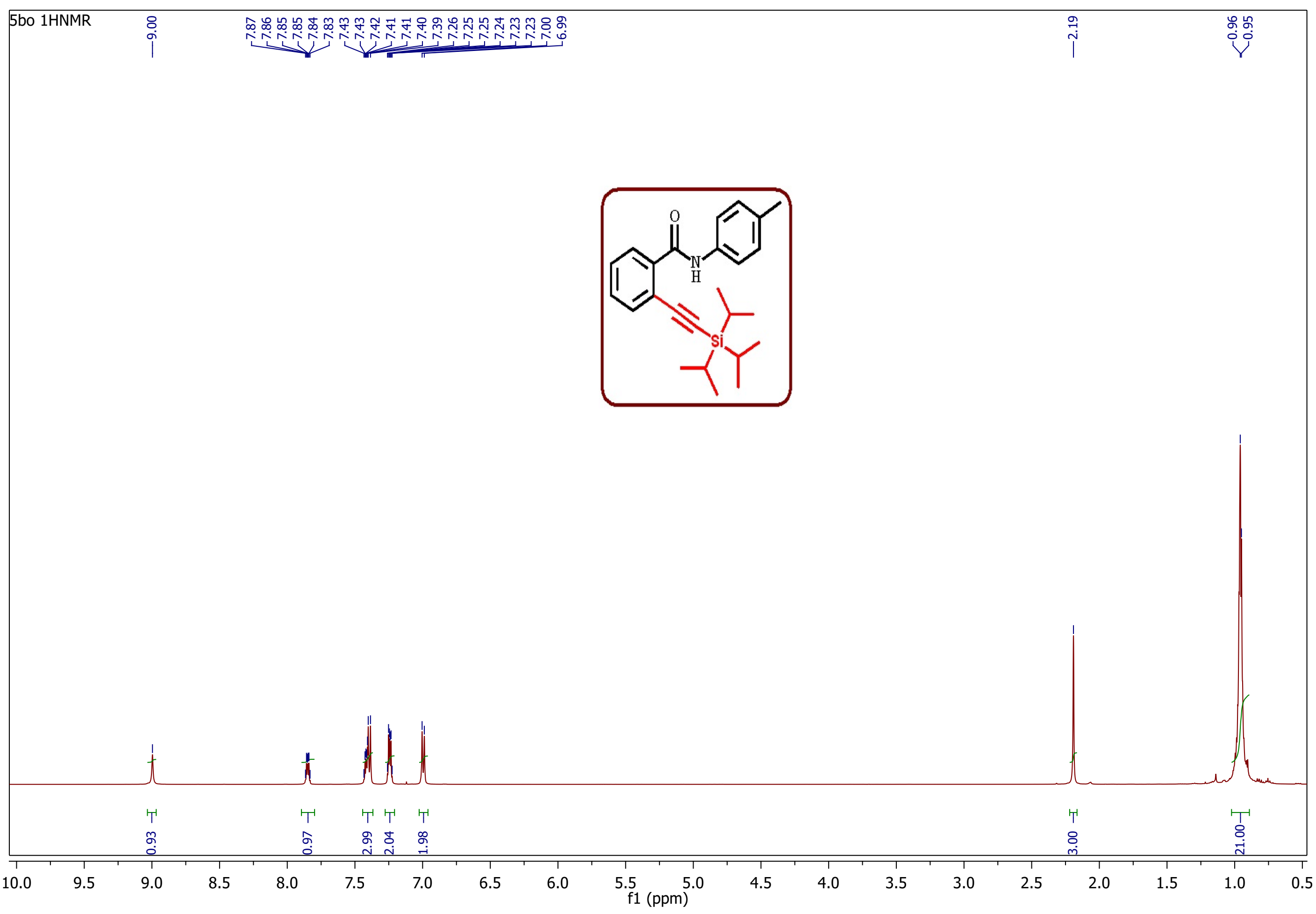





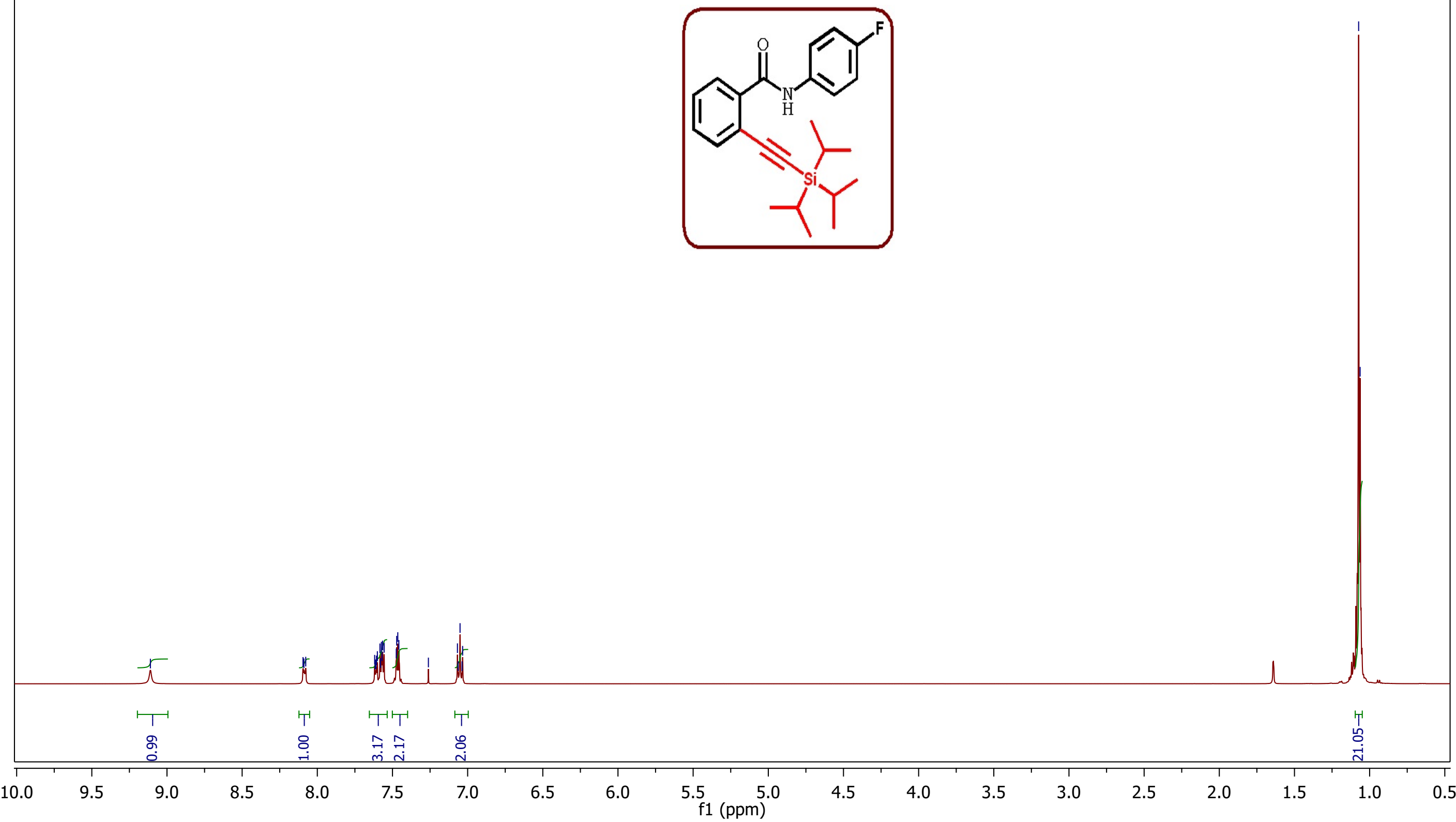


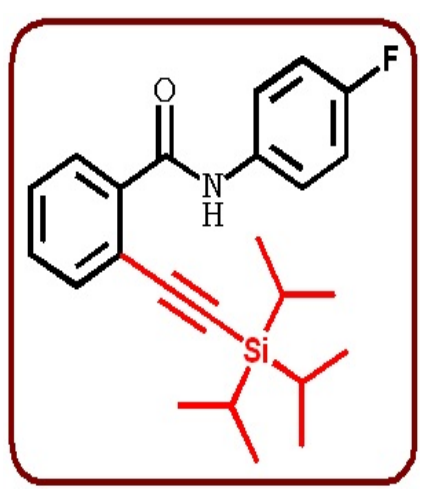



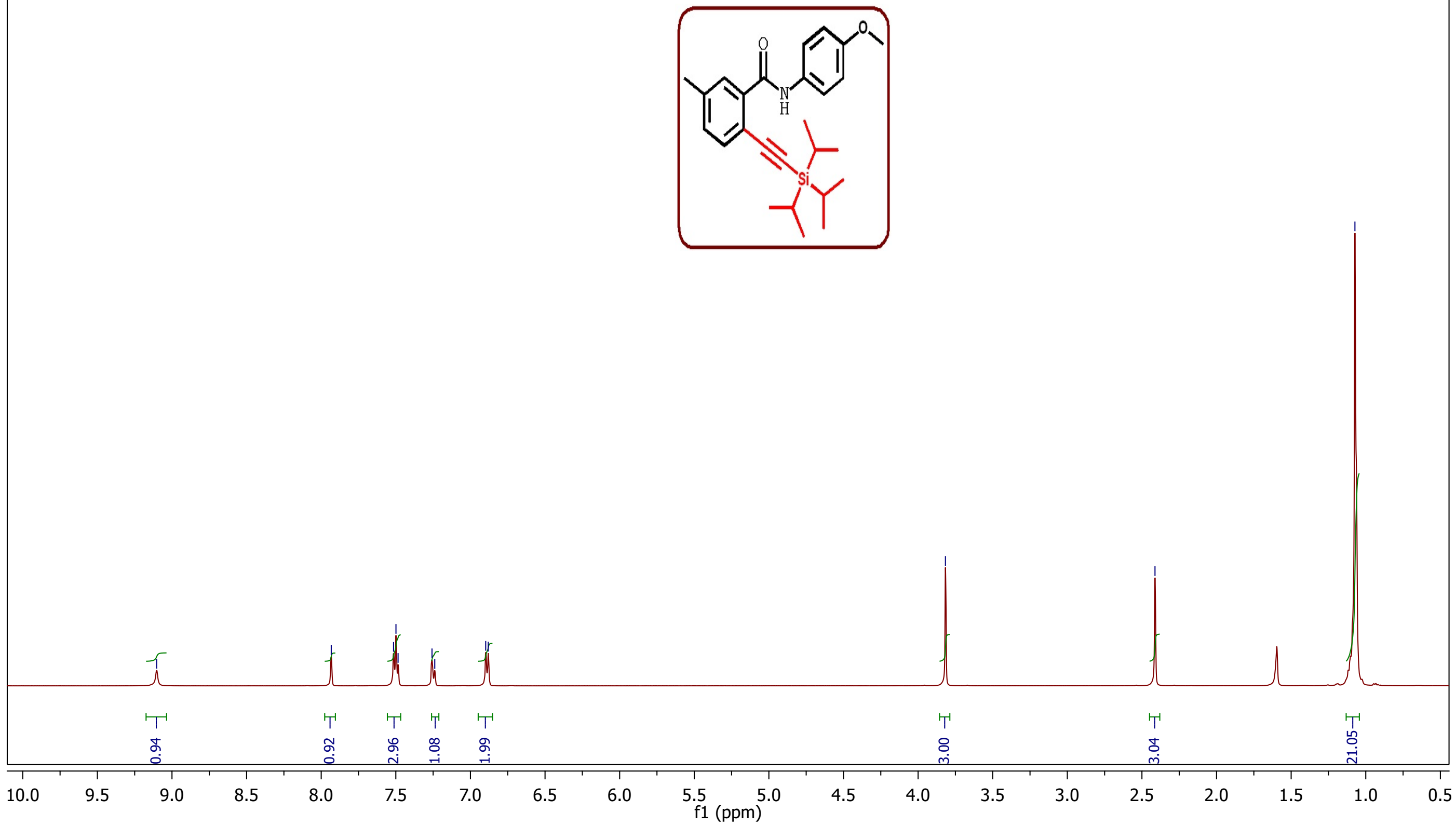

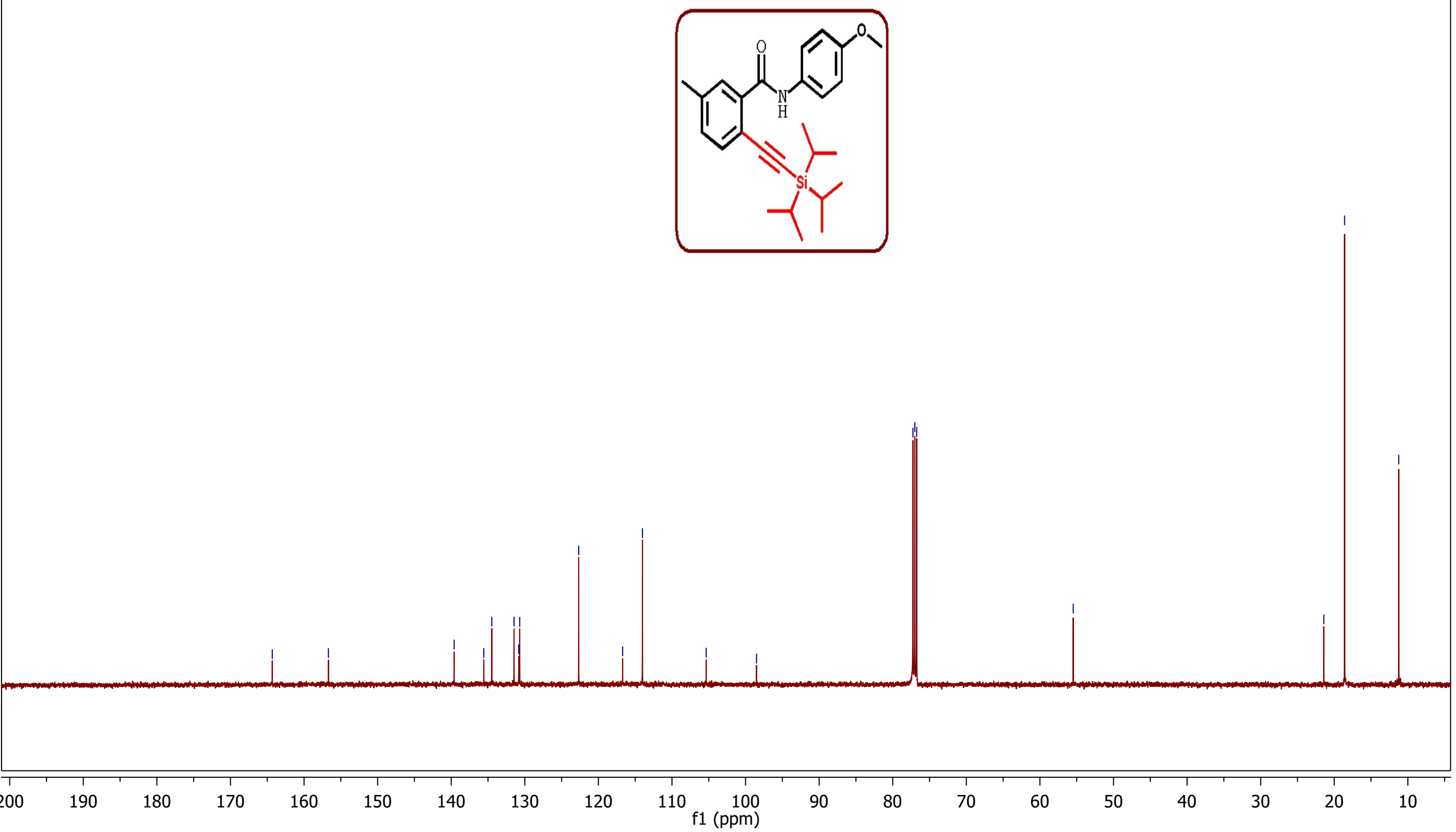


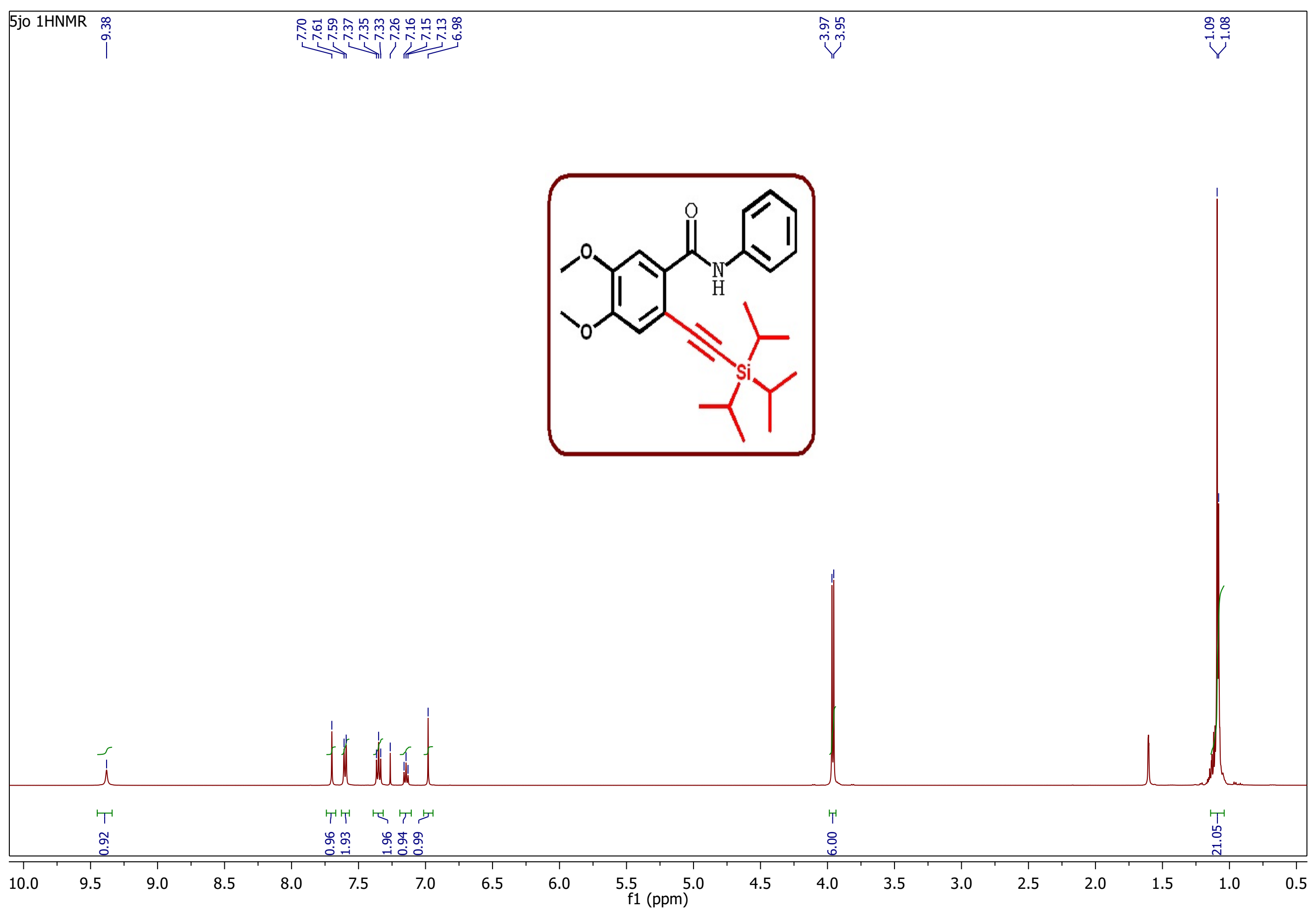




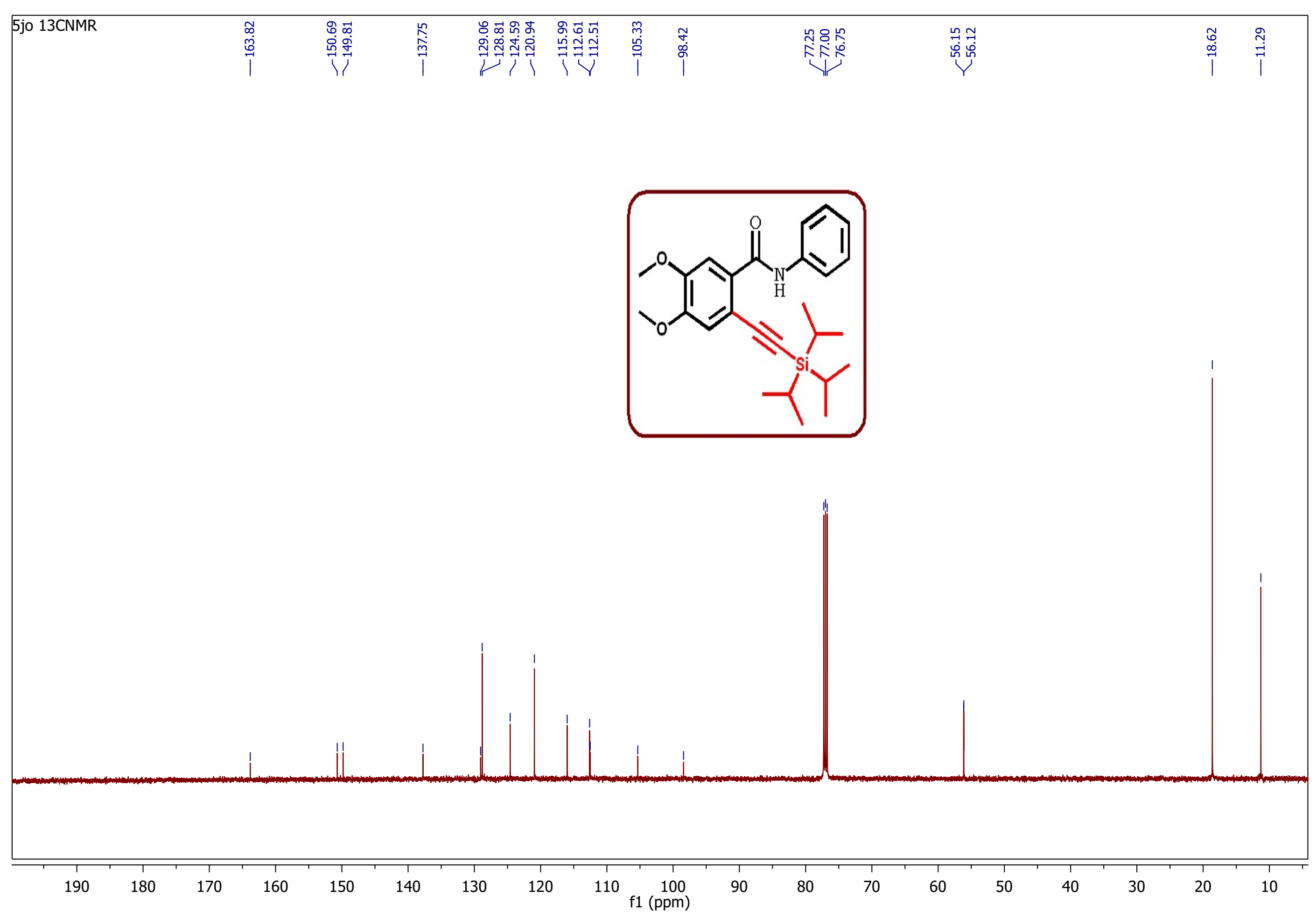




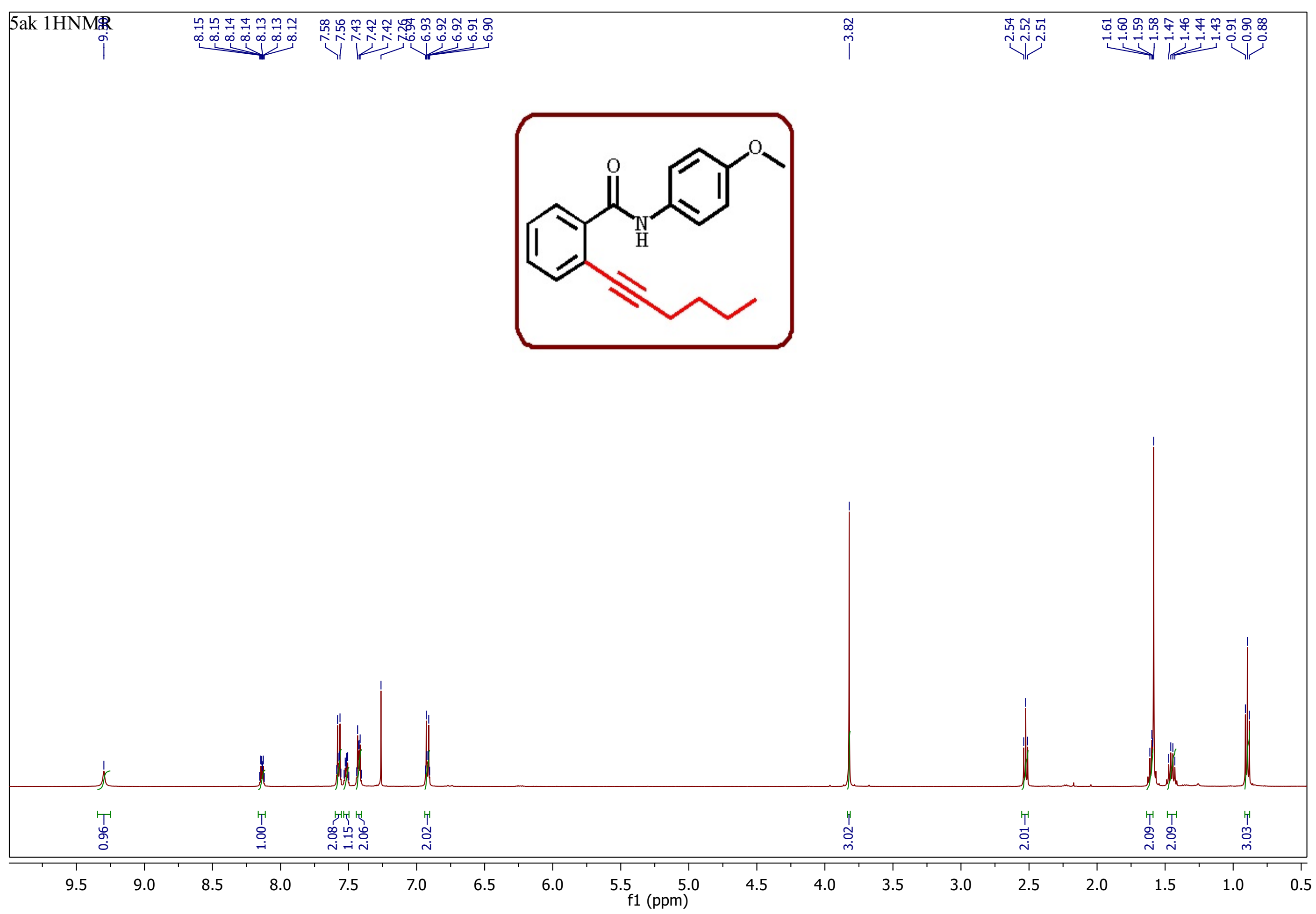




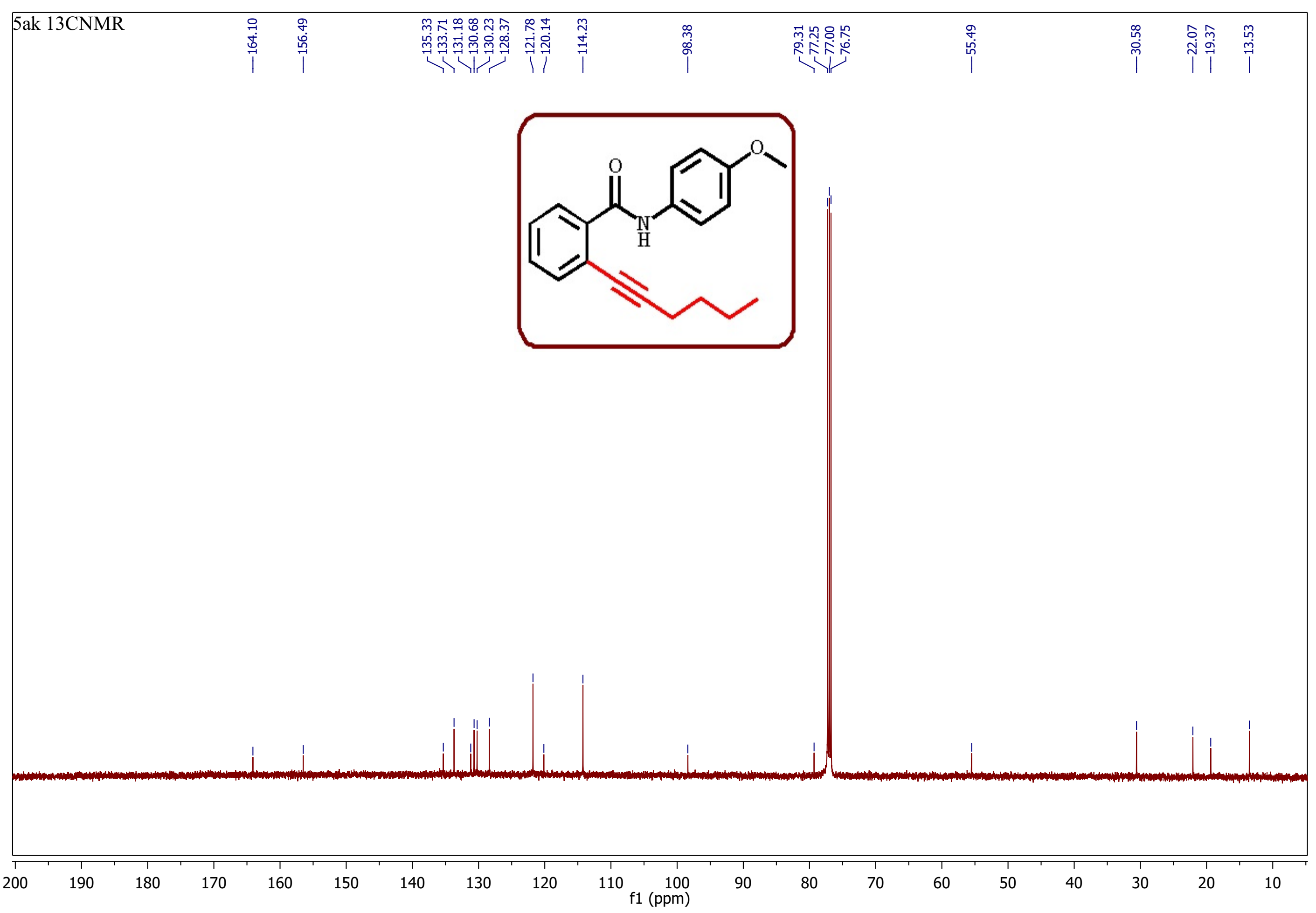




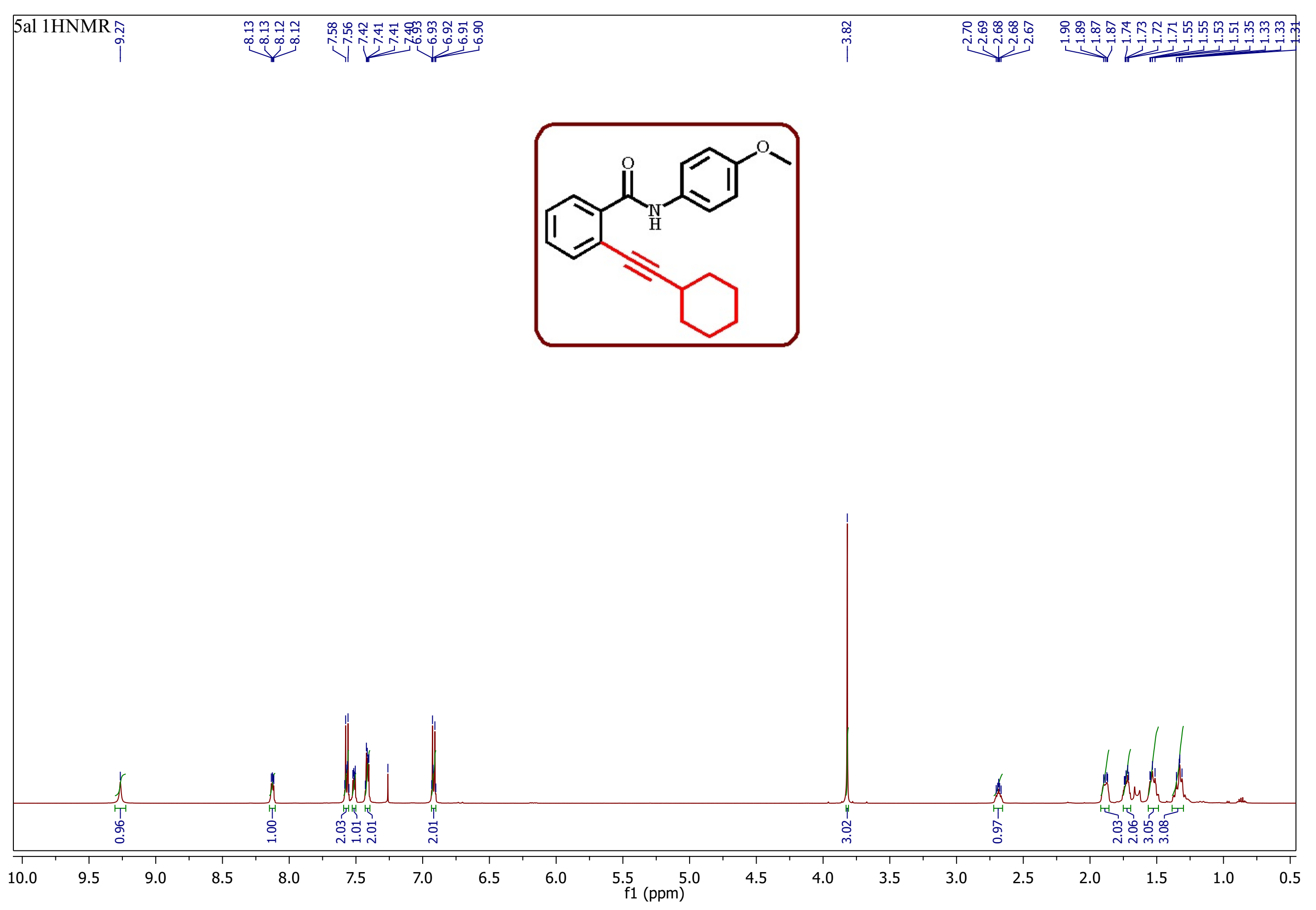




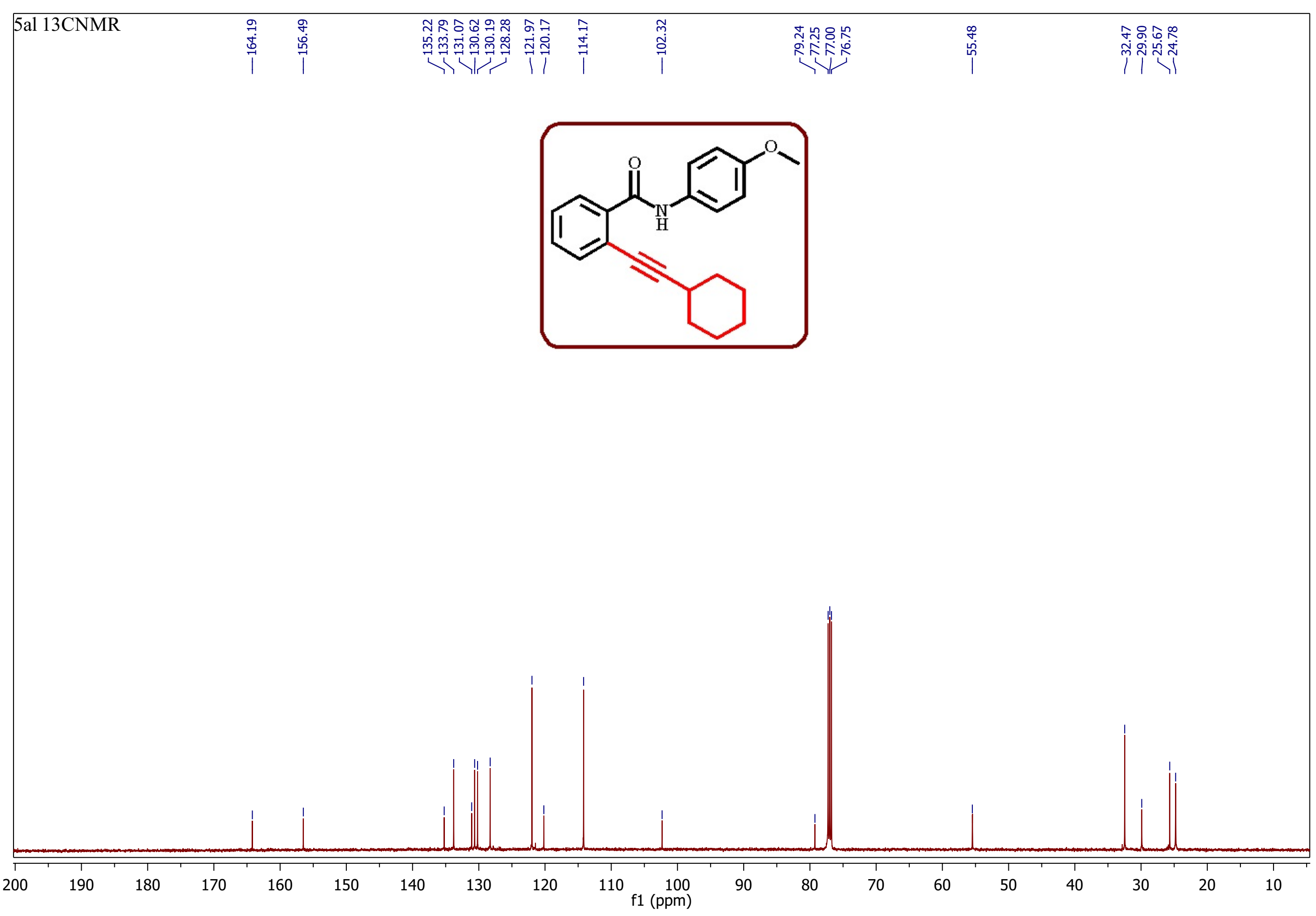




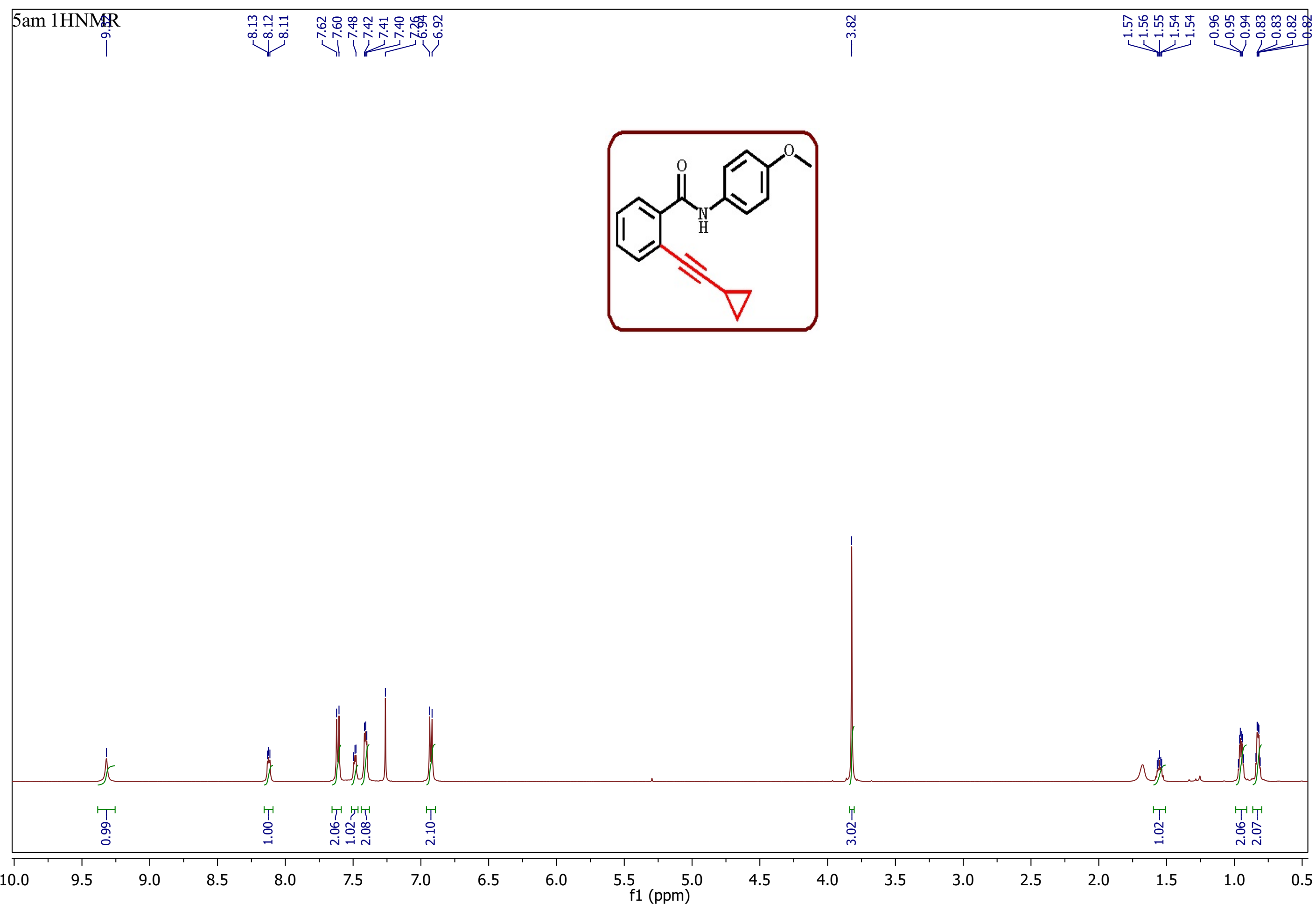




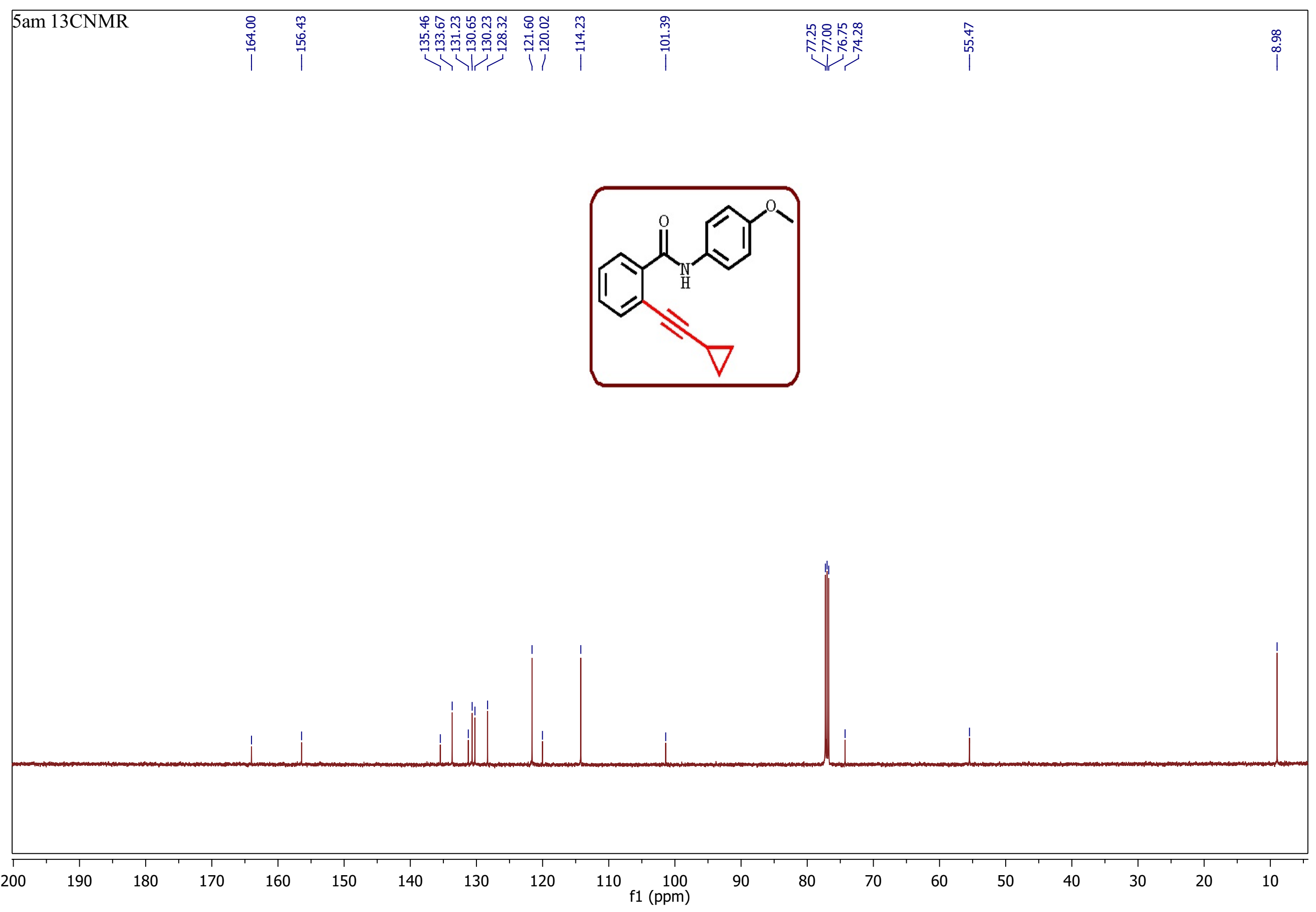




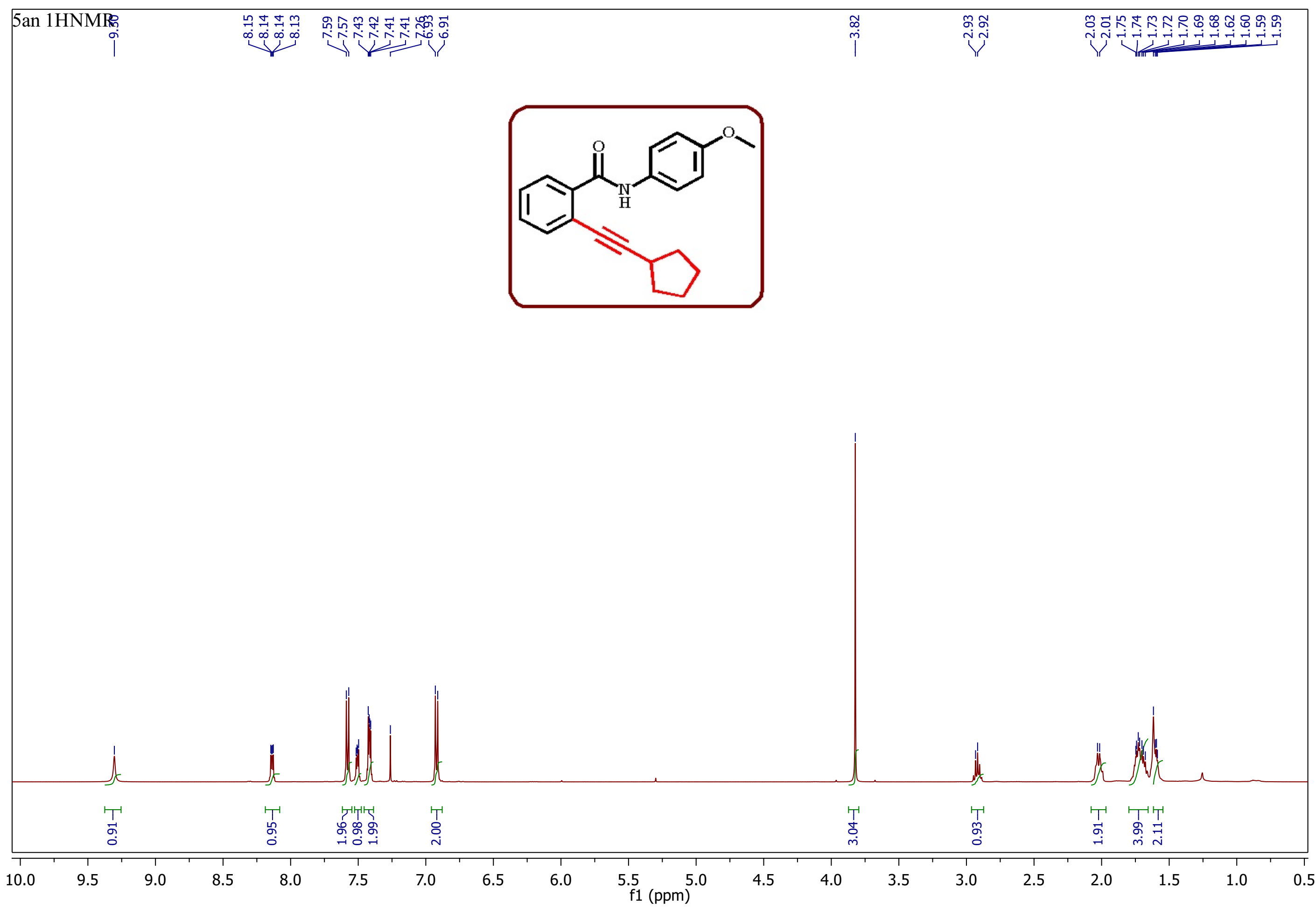








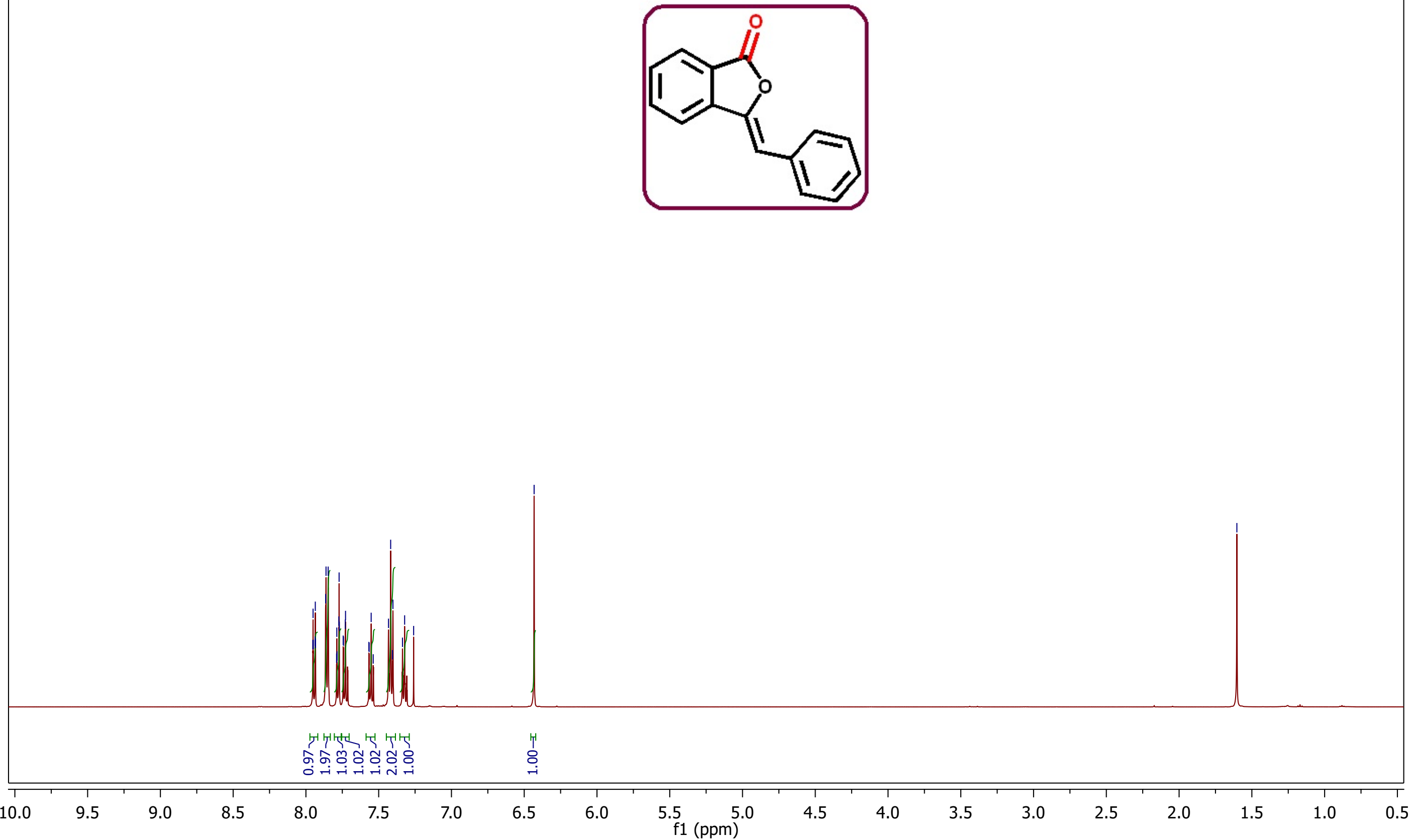


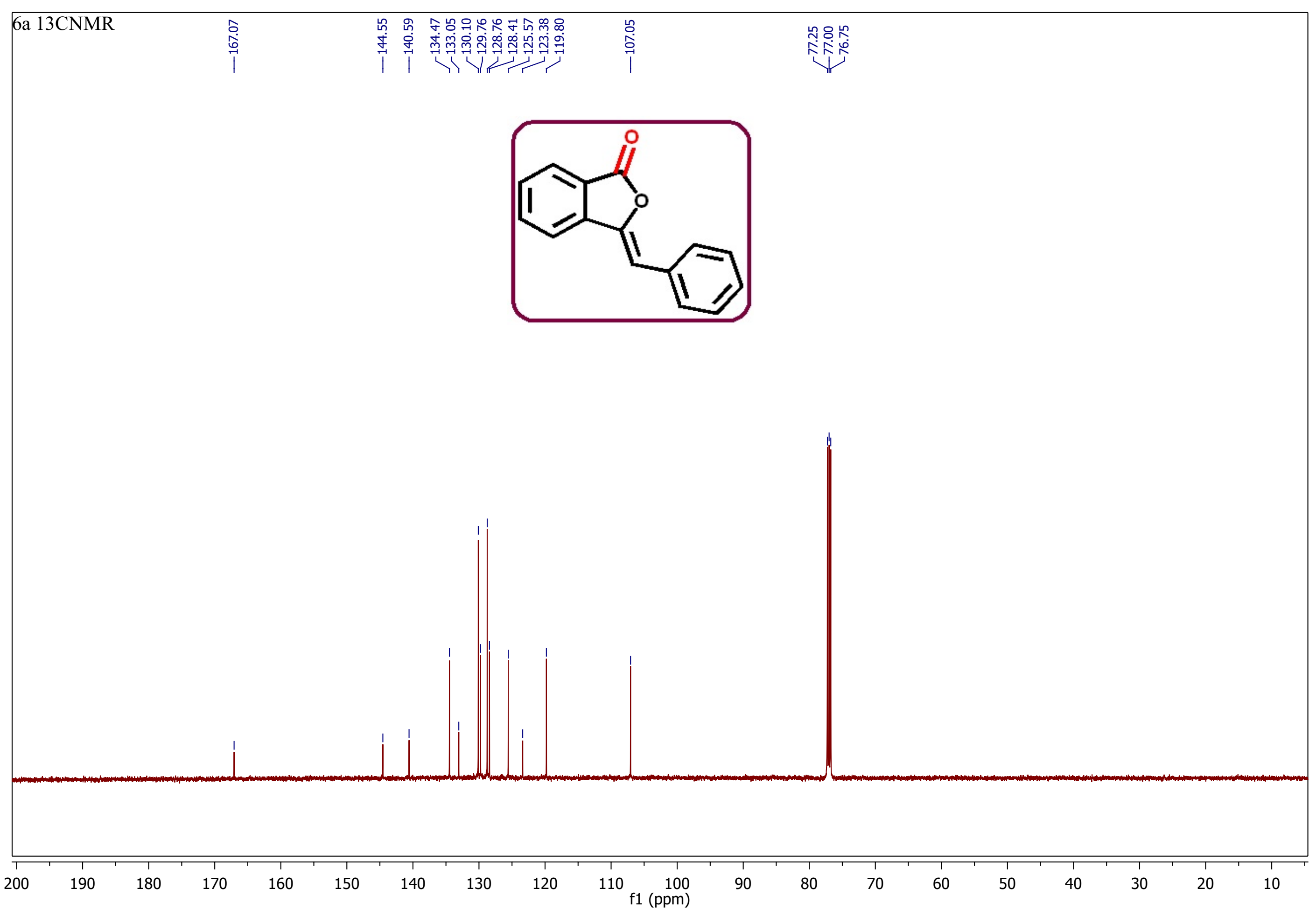




ofour





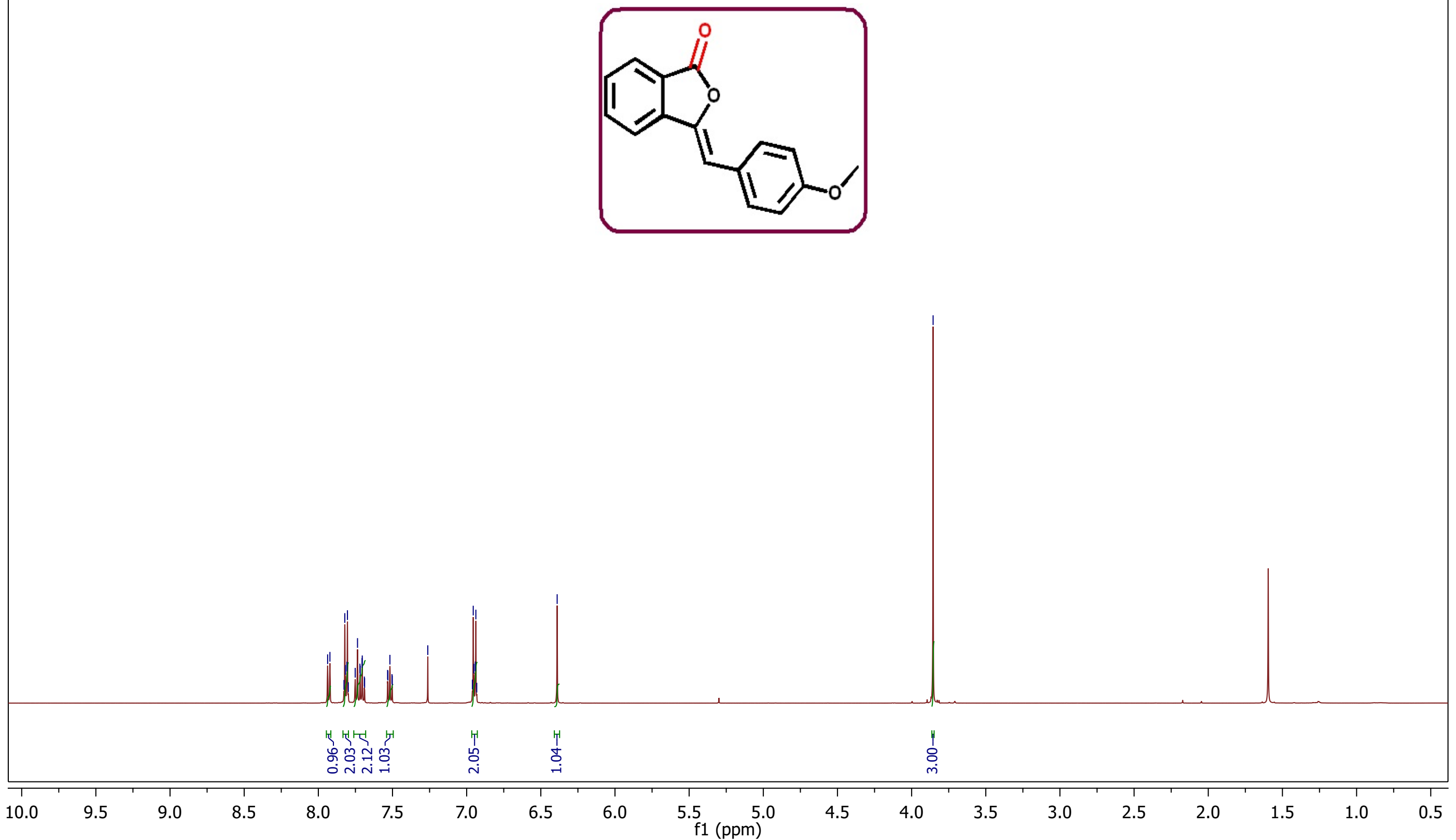







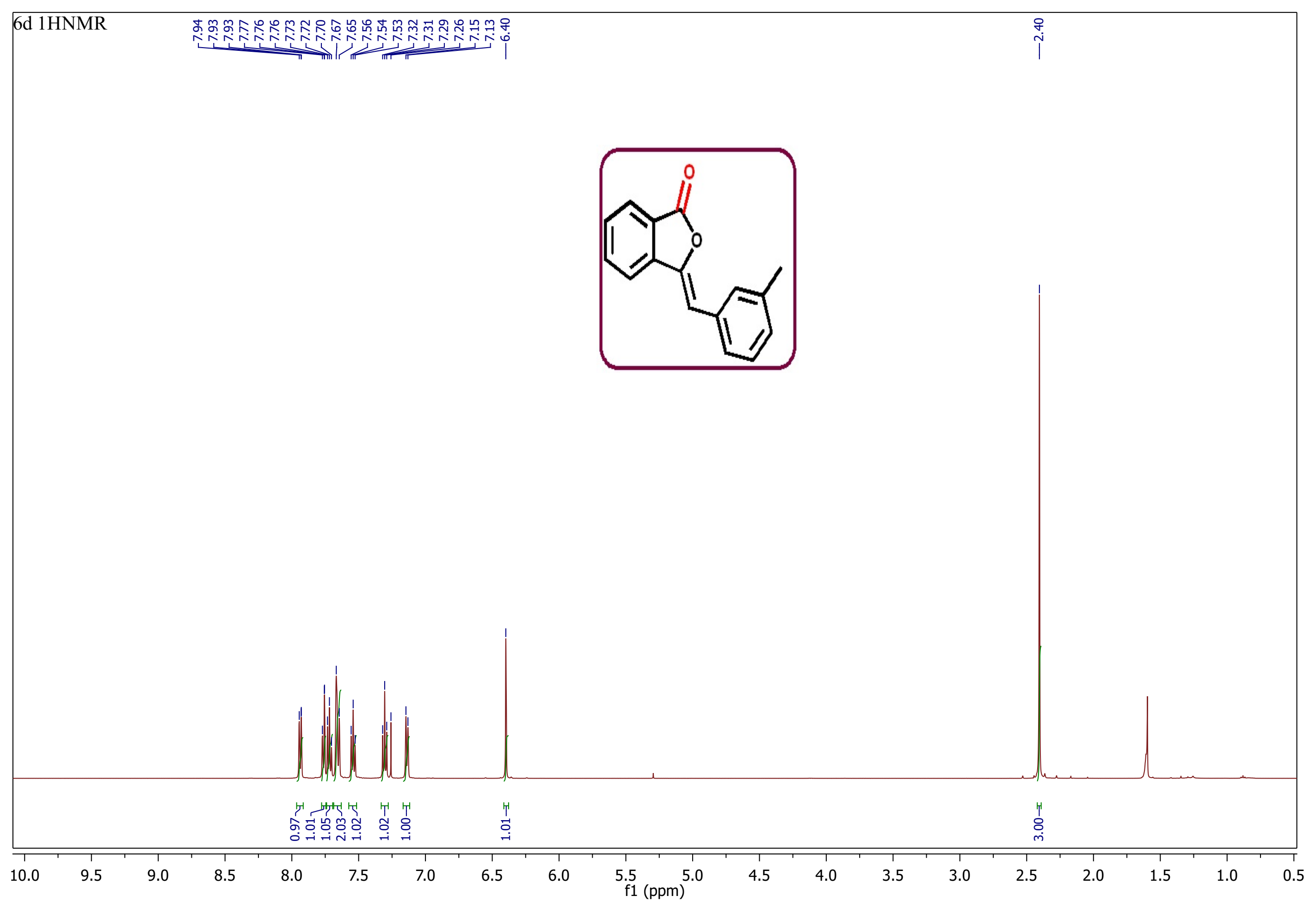




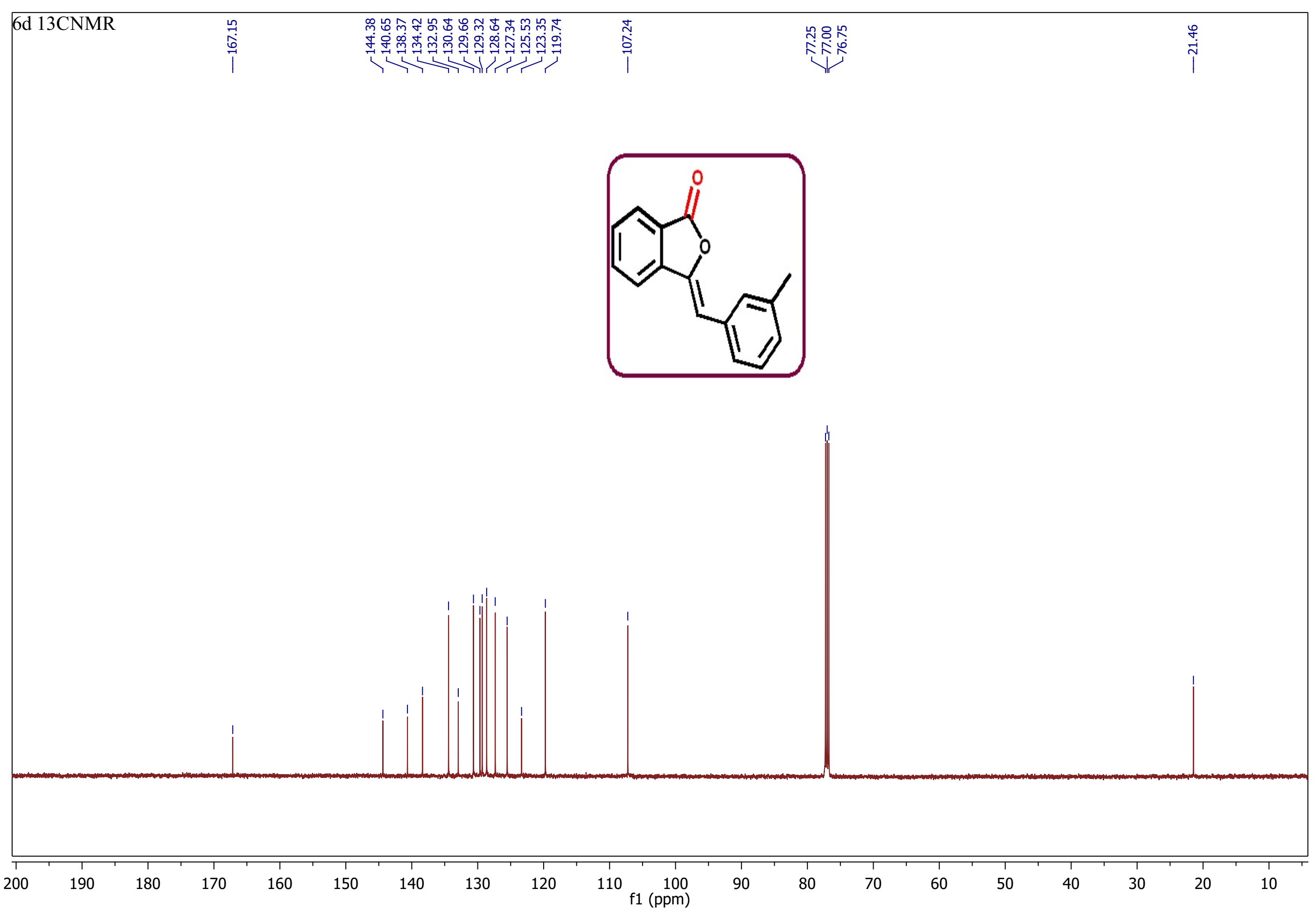














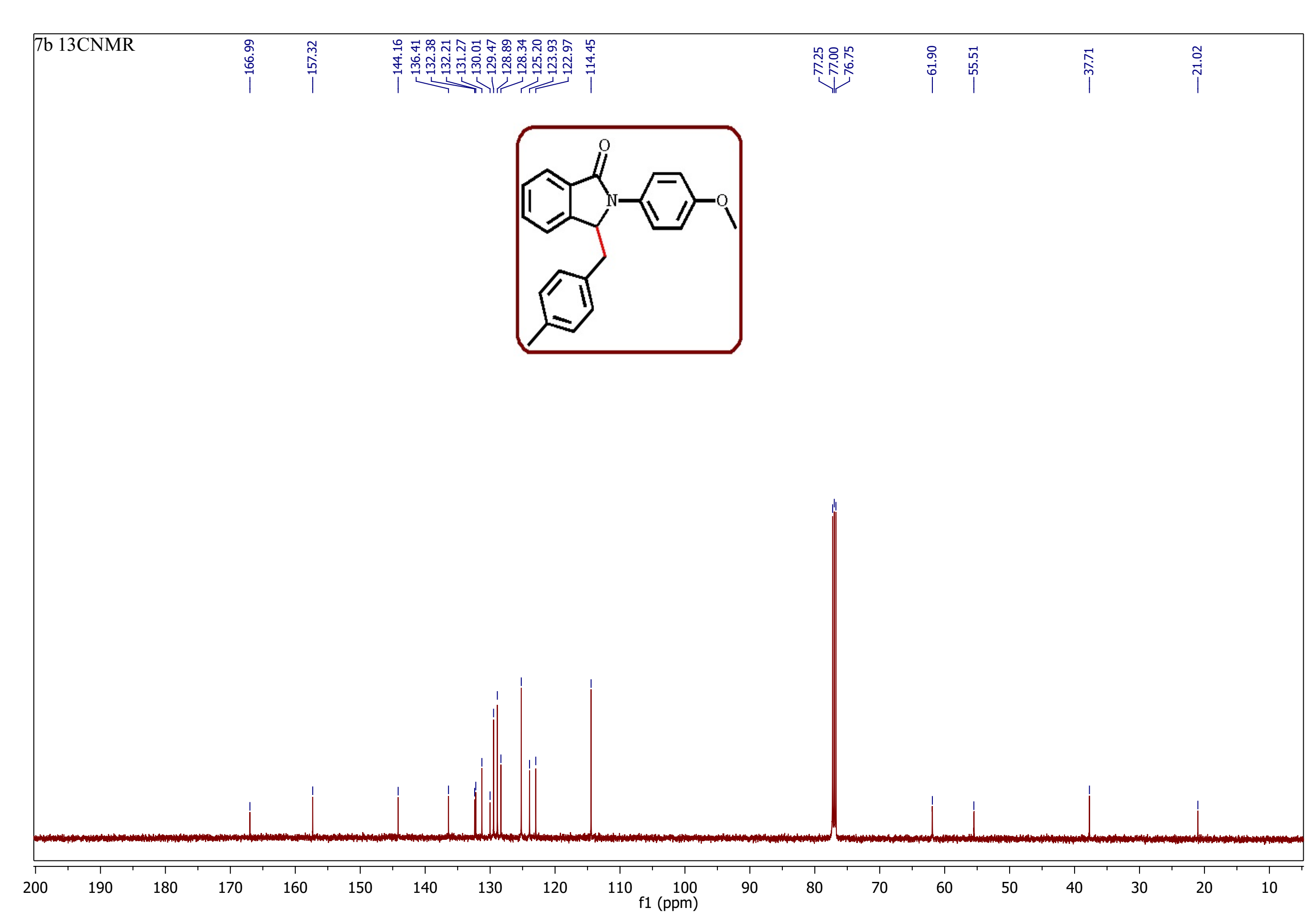






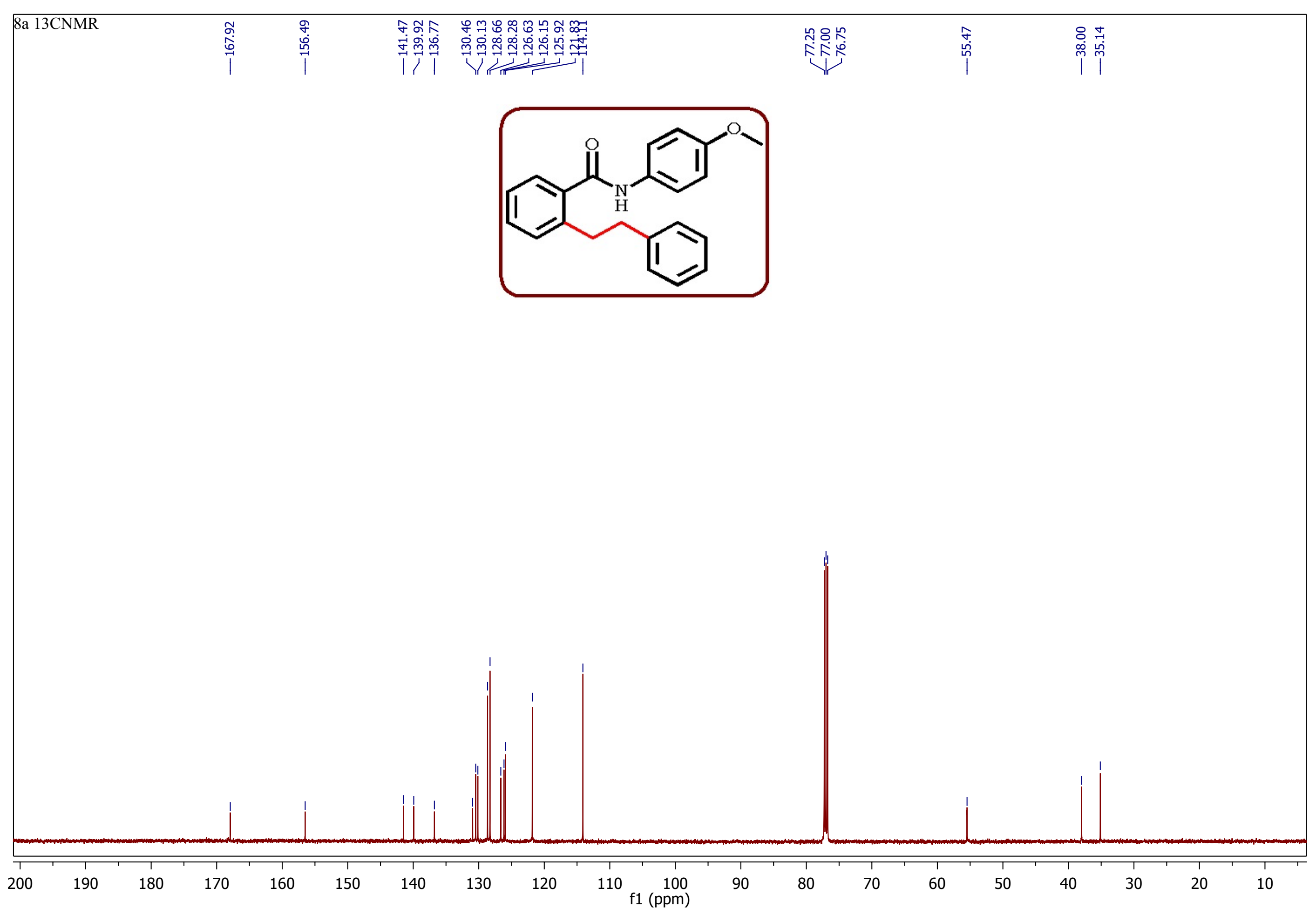



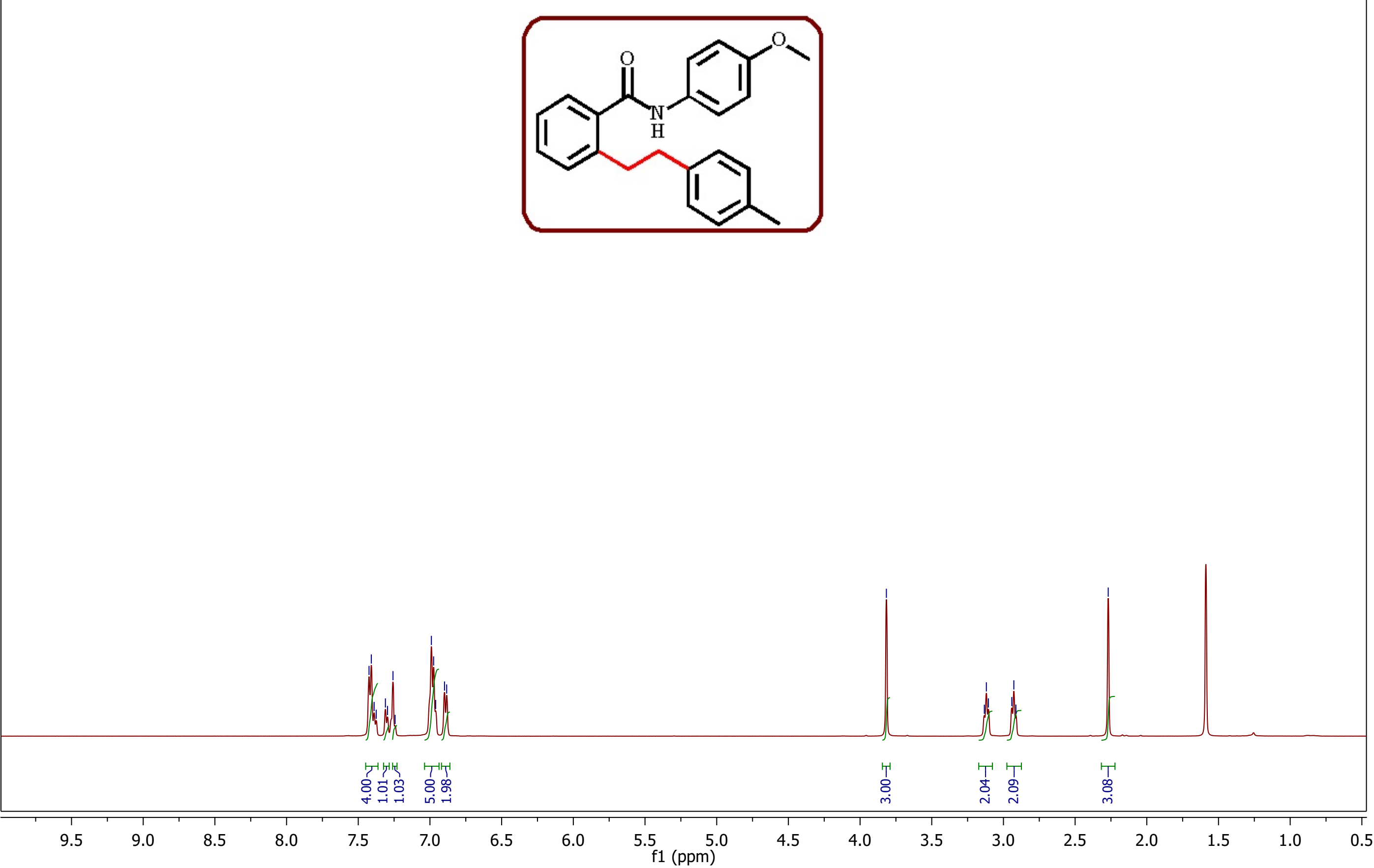


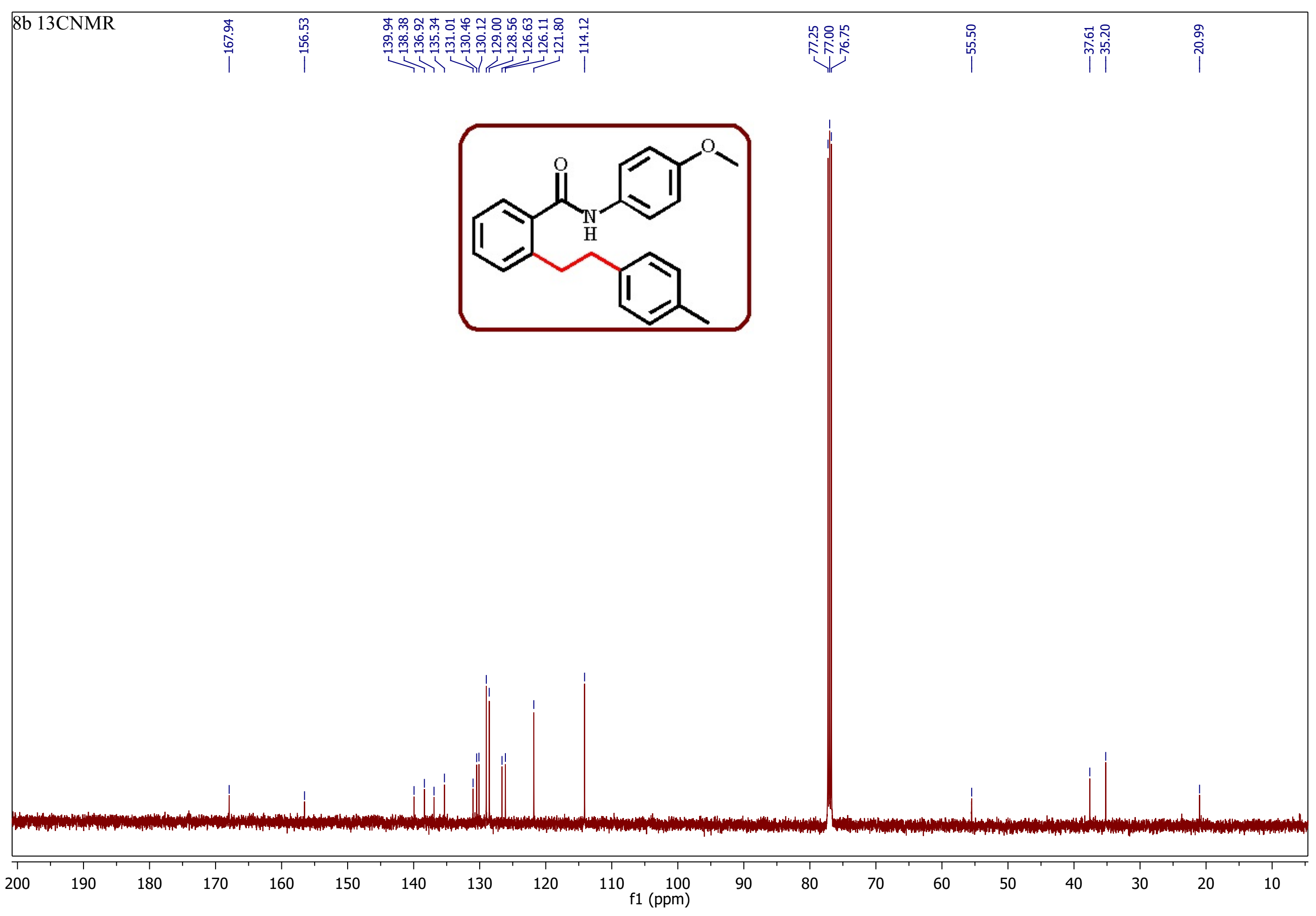

